\title{
Selective Thermoplasmonic Embedment of Supported Silver Nanocrystals
}

\author{
Ella Kirkland
}

A thesis submitted to the Faculty of Graduate and Postdoctoral Affairs In partial fulfillment of the requirements for the degree of

Master of Science

in

Chemistry

Carleton University Ottawa, Ontario

(C) 2020, Ella Kirkland 
The undersigned hereby recommends to the

Department of Chemistry

acceptance of the thesis

\title{
Selective Thermoplasmonic Embedment of Supported Silver Nanocrystals
}

\author{
Submitted by: \\ Ella Kirkland \\ In partial fulfillment of the requirements of the degree of \\ Master of Science
}

Dr. Anatoli lanoul

Supervisor 


\begin{abstract}
Noble metal nanocrystals are known for their unique optical and electronic properties which stem from their ability to support localized surface plasmon resonance (LSPR). These properties have made noble metal nanoparticles extremely popular for applications in sensors, imaging and spectroscopy techniques, and electronic and catalytic devices. In the past twenty years there has been an increase in the interest in the field of thermoplasmonics which focuses on harnessing the LSPR properties of nanoparticles for localized heating applications. The goal of this work was to investigate the selective thermoplasmonic excitation of different types of silver nanocube $(\mathrm{AgNC})$ structures in supported monolayers via thermoplasmonic embedment. AgNCs monolayers were deposited on polystyrene thin films using Langmuir-Schaefer deposition and then subject to different intervals of laser exposure. A wavelength of $458 \mathrm{~nm}$ was chosen to selectively embed the individual AgNCs and a wavelength of $568 \mathrm{~nm}$ was chosen to selectively embed the silver nanocube clusters (AgClusters). The extent of selective embedment was determined by analyzing the topographic profiles of the different AgNCs and AgClusters in the monolayer following laser exposure. Based on the embedment patterns achieved with the respective wavelengths, it was determined that both types of selective embedment were attained. In addition to being the first reported instance of spatially resolved thermoplasmonics in supported nanoparticle monolayers, these selective embedment techniques show promise for future applications in nanopatterning.
\end{abstract}




\section{Acknowledgements}

Firstly, I must extend my most sincere thanks to Dr. Daniel Prezgot for his continued patience with my endless litany of questions. Dan, you were instrumental to my learning and development as a researcher in this lab and without your support this thesis would not have been possible.

Secondly, I would like to thank Devron Colley for being the most outstanding lab mate one could ever ask for. Your constant encouragement, playful sense of humour, and scintillating discussions made work more fun than the hours in between.

To all of the current and past students at Carleton with whom I have had the pleasure of sharing classes and labs, thank you for making my time at Carleton the most fun years of my life. I must extend a very special thank-you to Fiona Ebanks for deciding to become friends with the "girl with yellow sandals who always wears shorts" and to Emma Wistaff for deciding to come to Carleton for her graduate studies. You gals are awesome scientists and my number-one hype team. I am so incredibly grateful for your friendship.

I want to thank Maria DeRosa and Seán Barry for being terrific professors and giving me my first research opportunities at Carleton. You two were instrumental in inspiring my love for chemistry and nanotechnology and I will always cherish the time I spent in each of your labs. I must also thank Chantelle Gravelle for making the main office in Steacie one of my favourite places to hang out and always lending a hand when I needed assistance with administrative stuff (which was frequently). If it weren't for you, I would never have kept on top of all the important deadlines and probably wouldn't even have made it to this point. I would also like to thank Peter Mosher for keeping the Carleton Chemistry Department running, JJ for making my TEM imaging sessions highly entertaining, and Daniel Sun and Kyle Fournier for making TAing my favourite part of grad school.

I would like to thank Bob Burk, not only for inspiring me to switch into chemistry in my first year of undergrad but also for his continued kindness and advice throughout my time in Carleton. Bob you were always willing to listen to any problem I was having, science related or not, and I found comfort in your office on my gloomiest days. I can say without a shred of doubt that having you as a teacher has changed my life for the better.

This thesis would not have been possible without the support of my family and friends. To my Mom and Dad, thank you giving me the gift of education and providing me with a place to eat, sleep and seek comfort even though I am well beyond the age at which most children move out. To my grandparents Omi and Papa, thank you for being the biggest cheerleaders of my accomplishments no matter how small. To my baby sister, Annie, thank you for making me laugh so hard I cry every time I needed it most and for always reminding me that I could do things even when I had lost faith in myself. I love you to the moon and back. To all of my non-chemistry friends who always cheered me on no matter how much or how little of my work you understood, thank you for your earnest interest and constant support. To my darling boyfriend Bryson, thank 
you for listening to my explanation of plasmonics and FDTD simulations like an absolute champion and inspiring me with your relentless work ethic.

Last, but most certainly not least, I would like to thank Dr. Anatoli lanoul from the bottom of my heart. Your vast knowledge is rivalled only by your incredible generosity and your dedication to your students. I am extremely grateful that you were willing to take me on as a graduate researcher when I came to you half-way through my degree in desperation. Your hands-on approach to research as a supervisor is unparalleled and it was a privilege to be able to work with you these past 16 months. I truly cannot thank you enough. In the words of Dan Prezgot "you may have us working with silver, but you have a heart of gold." 


\section{List of Abbreviations}

AFM - Atomic Force Microscope or Atomic Force Microscopy

AgNC - Silver nanocube

AgCluster- Silver nanocube cluster

CW- Continuous Wave

DOPC-1,2-dioleoyl-sn-glycero-3- phosphocholine

EG - Ethylene glycol

EM-Electromagnetic

EEF- Electrical Field Enhancement Factor

FDTD - Finite Difference Time-Domain

GA-Glycoaldehyde

$\mathrm{k}_{\mathrm{c}}$ - Spring constant

LB- Langmuir Blodgett

LS-Langmuir Schaefer

LSP-Localized Surface Plasmon

LSPR - Localized Surface Plasmon Resonance

PS - Polystyrene

PSP-Propagating Surface Plasmon

PR-Plasmon Resonance

PTFE - Polytetrafluoroethylene

PVP - Poly(vinyl)pyrrolidone

rpm- Revolutions Per Minute

SPR - Surface Plasmon Resonance

TEM- Transmission Electron Microscope or Transmission Electron Microscopy

TFSF- Total-field scattered-field

UV-Vis-Ultraviolet-Visible 


\section{Table of Contents}

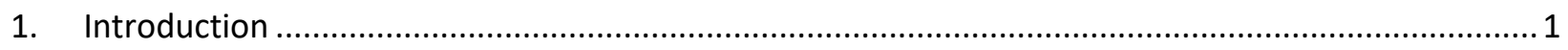

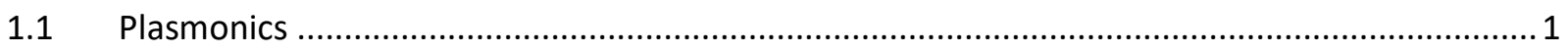

1.1.1 Bulk, Surface and Localized Plasmons......................................................................... 2

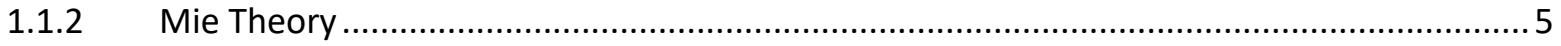

1.1.3 Effect of Chemical Composition on LSPR …................................................................ 9

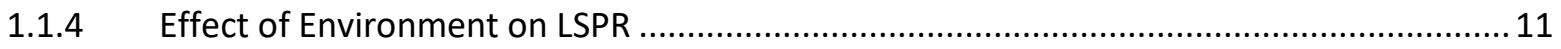

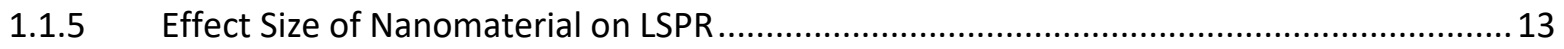

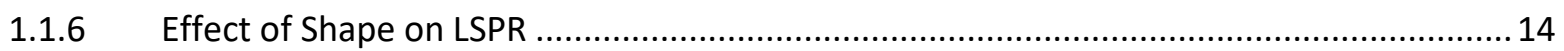

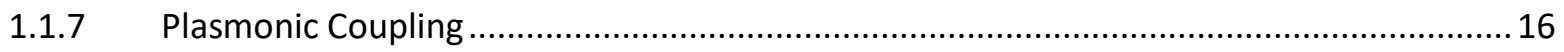

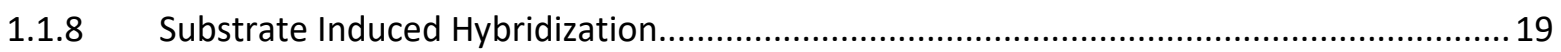

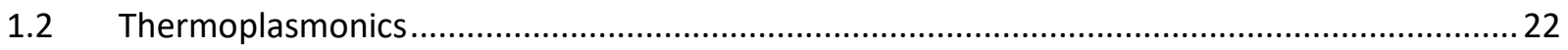

1.2.1 Mechanism of Photothermal Heat Generation in Nanoparticles .....................................23

1.2.2 Temperature Increase of Thermoplasmonic Particles.................................................. 24

1.2.3 Effect of Composition, Size, and Shape on Thermoplasmonic Properties........................ 25

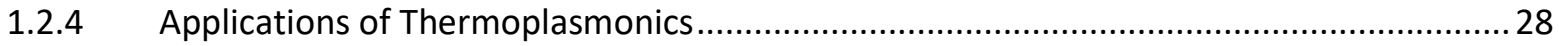

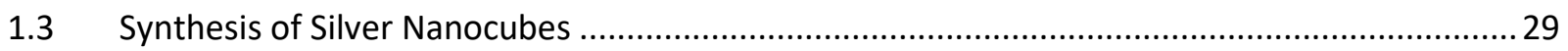

1.3.1 Heating of Ethylene Glycol and Reduction of Silver ....................................................... 30

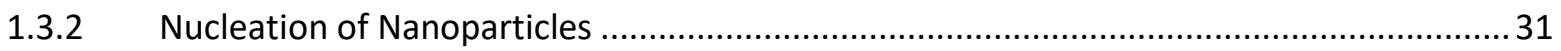

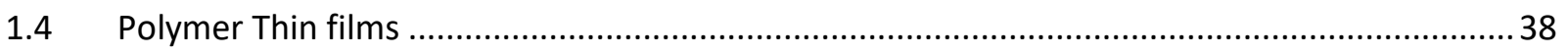

1.4.1 Nanoparticle Polymer Thin-Film Composites ............................................................... 39

1.4.2 Embedment of Nanoparticles in Polymer Thin-Films .................................................. 41

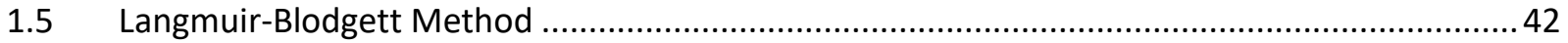

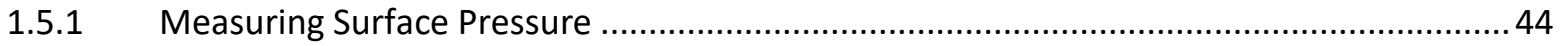

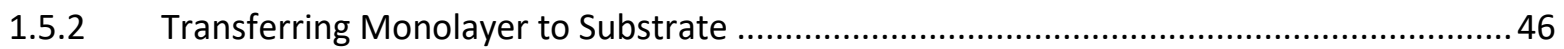

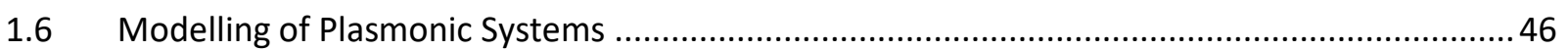

1.6.1 Finite Difference Time Domain Modelling Method ....................................................... 47

1.6.2 Discrepancies Between Experimental Conditions and Modelled Conditions .................... 49

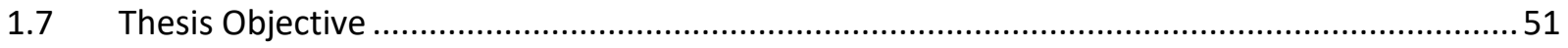

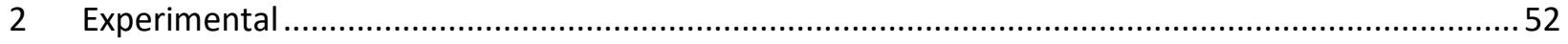

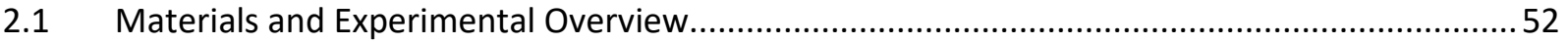

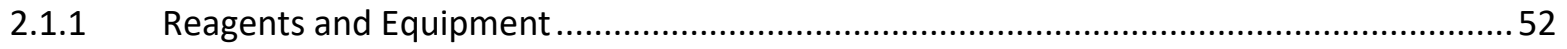

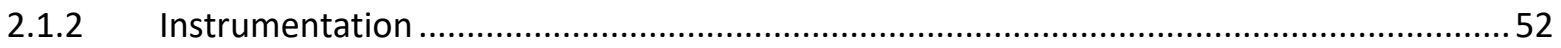




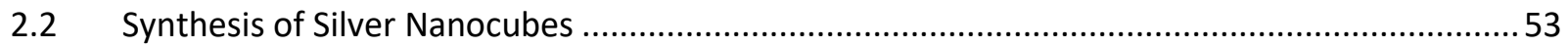

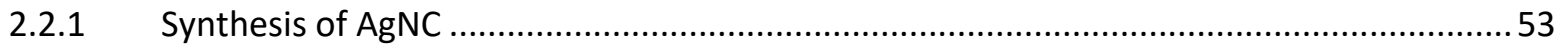

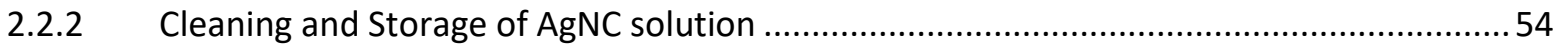

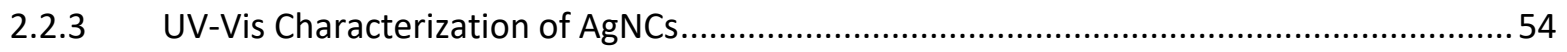

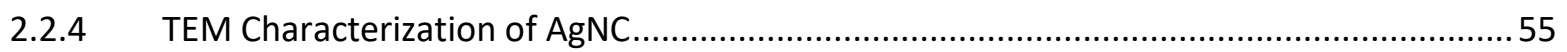

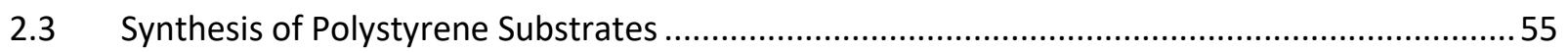

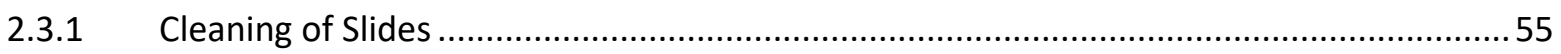

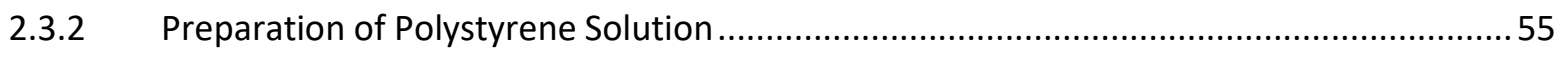

2.3.3 Spin Coating the Glass Slides with Polystyrene ........................................................... 55

2.3.4 Characterizing Surface Roughness and Film Thickness with AFM ..................................56

2.4 Synthesis of Silver Nanocube Monolayers via Langmuir-Schaeffer Deposition ........................56

2.4.1 Cleaning the Langmuir-Schaeffer Trough .................................................................. 56

2.4.2 Preparing the AgNC solution for Langmuir-Schaeffer Deposition ...................................56

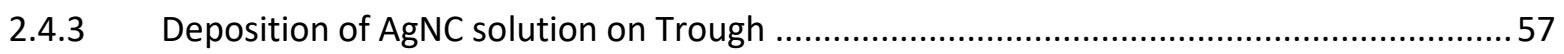

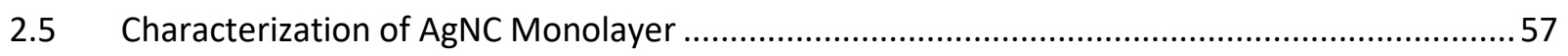

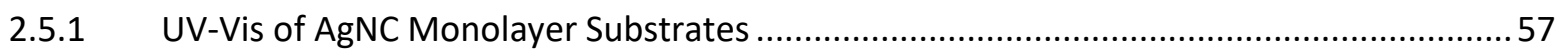

2.5.2 Characterization of Monolayer by Atomic Force Microscopy .......................................... 58

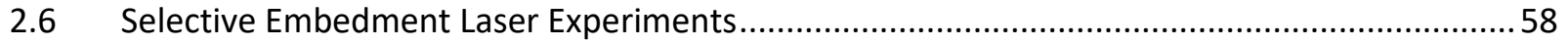

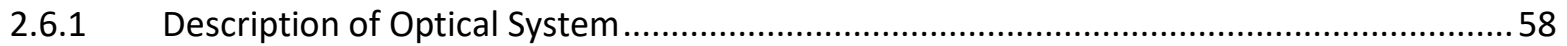

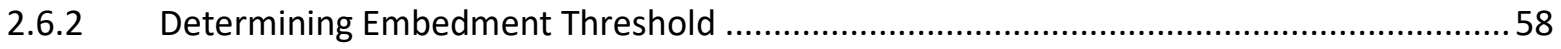

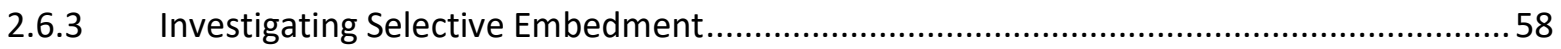

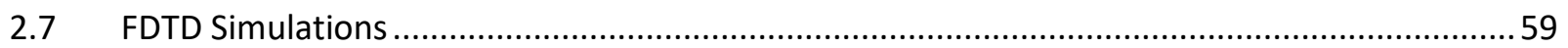

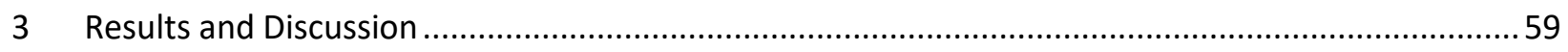

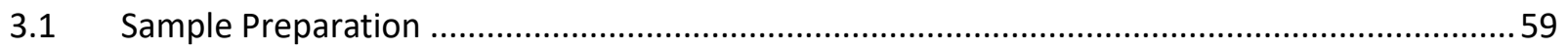

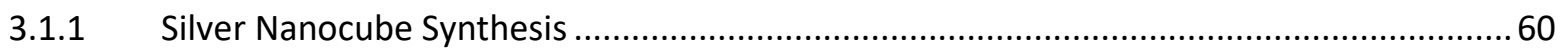

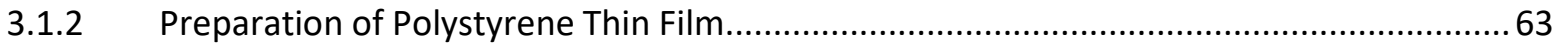

3.1.3 Deposition of AgNC on to PS Thin Films via Langmuir Shaefer Deposition ......................64

3.2 Determination of Laser conditions for Selective Thermoplasmonic Embedment....................66

3.2.1 Laser Conditions Required for Selective Thermoplasmonic Embedment of Individual

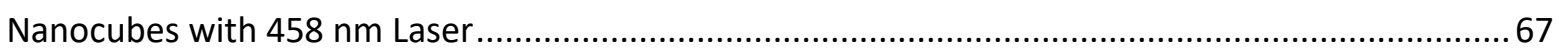

3.2.2 Laser Conditions Required for Selective Thermoplasmonic Embedment of Nanocube Clusters 67

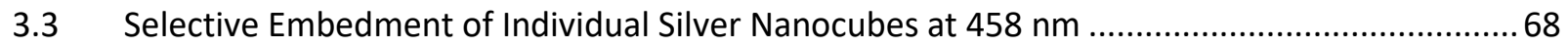

3.4 Selective Embedment of Silver Nanocubes Clusters at $568 \mathrm{~nm}$.......................................... 73 
3.4.1 Measurement of Height Change Selective Embedment of Cluster Experiments ............... 75

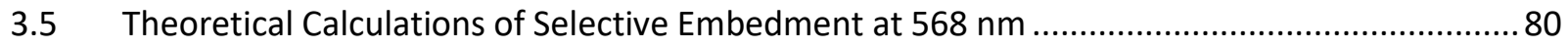

3.5.1 Calculated Absorption Cross section of Different Clusters at $568 \mathrm{~nm}$............................. 81

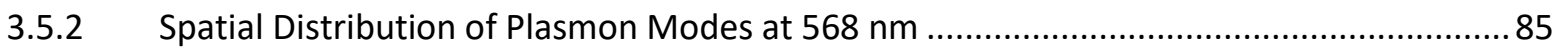

3.6 Factors that Potentially Affected the Selective Embedment of Clusters at $568 \mathrm{~nm}$.................87

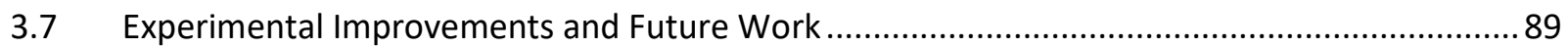

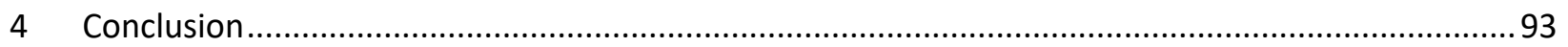

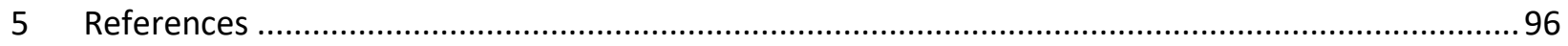

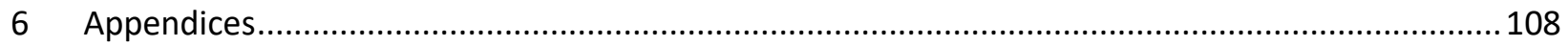

6.1 Supplementary Material for Section 3.1

6.1.1 Additional Transmission Electron Microscopy Characterization of AgNCs in Solution ....108

6.1.2 Thickness Measurement of PS Thin Film ................................................................ 108

6.1.3 Additional Atomic Force Microscope and UV-Vis Characterization of Supported AgNC

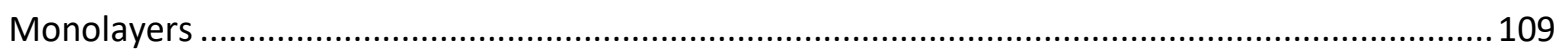

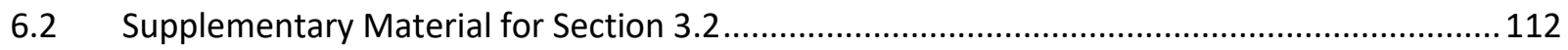

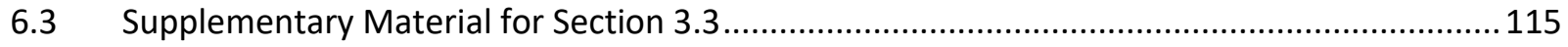

6.3.1 Series of AFM Images Taken Before and After Laser Exposure at $458 \mathrm{~nm}$.....................115

6.3.2 Zoomed in Regions of AFM images Taken After Laser Embedment Intervals .................127

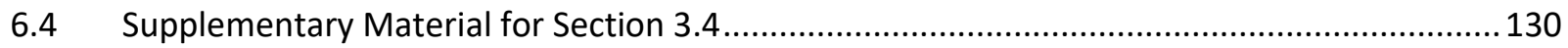

6.4.1 Regions of the AgNC Monolayer Investigated for Selective Embedment of Nanocube Clusters at $568 \mathrm{~nm}$

6.4.2 Cross Sections of AgNC Monolayer in Regions A, B and C used for Height Measurement

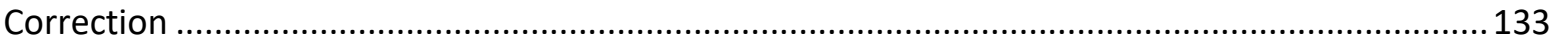

6.4.3 Height Measurement of AgNCs in Regions A, B and C.............................................136

6.5 Supplementary Material for Section 3.5 ......................................................................... 141

6.5.1 FDTD Calculated Absorption Spectra for Different Sizes of AgNC and Different Shapes and

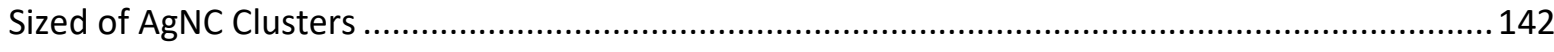

6.5.2 Different 2D-Planes Used for the E-Field Enhancement (EEF) Images of AgNC Clusters .145

6.5.3 EEF Images for Different Sizes and Shaped of Nanocube Clusters at $568 \mathrm{~nm}$................147

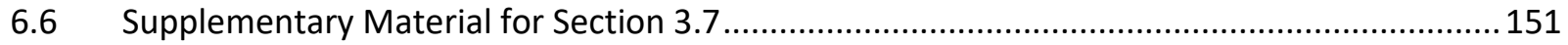

6.6.1 E-Field Enhancement Images of Different Sizes of Nanocube Clusters for at Their Peak

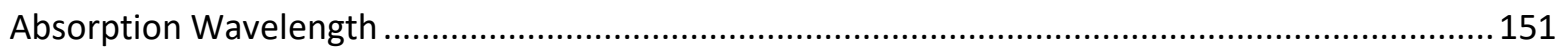




\section{List of Figures}

Figure 1.1: Schematic of Surface Plasmon resonance........................................................................... 3

Figure 1.2: Schematic of nanoparticle dipole generated by Localized Surface Plasmon resonance............. 5 Figure 1.3: Plot of the (A) real, $\boldsymbol{\varepsilon} r$, and (B) imaginary, $\boldsymbol{\varepsilon} \boldsymbol{i}$, components of the dielectric function of $A g, A u$, and $\mathrm{Si}$ as a function of wavelength. Reproduced with permission from $\left({ }^{19}\right)$..

Figure 1.4: Quality factor (Q) of the LSPR for a metal/air interface. A higher $Q$ denotes less damping and a stronger plasmon resonance. The shaded area represents the region of interest for many plasmonic applications.

Figure 1.5: Extinction spectra of the Ag nanocubes acquired by dispersing the nanocubes in the different solvent mixtures. (b) Shifts of the different plasmon resonance modes of the Ag nanocubes as a function of the refractive index. (c) Extinction spectra of Au nanocubes acquired by dispersing the nanocubes in the different solvent mixtures. (d) Shifts of the plasmon mode of the Au nanocubes as a function of the refractive index. The numbers in (b) and (d) indicate the different plasmon modes as in Figure $2 a$ and $b$. (e) Comparison of the refractive index sensitivity of the dipolar plasmon mode between the Ag and $\mathrm{Au}$ nanocubes. The solid and hollow symbols represent the measured and calculated plasmon shifts, respectively. The lines are linear fits.

Figure 1.6: Extinction efficiencies as a function of the wavelength of the incident light of a silver cube, different truncated cubes, and a spherical nanoparticle.

Figure 1.7: Plasmon hybridization scheme of longitudinal dipolar modes of an interacting nanoparticle dimer. Two interacting modes will mix to form an asymmetrically coupled anti-bonding mode and symmetrically coupled bonding mode.....

Figure 1.8: DDA calculated extinction spectra for the AgNCs in (A) Face-Face and (B) Edge-Edge orientations at varying separation distances of the dimer $(2,4,8,10$, and $20 \mathrm{~nm})$. The incident light is polarized along the interparticle axis.

Figure 1.9: Schematic illustration of (a) intrinsic cube modes with induced hybridization from the dielectric; (b) energy diagram of dielectric effect where the black dotted lines represent the pure dielectric screening effect and the thin blue lines represent the substrate mediated interaction; (c) charge distribution of fully hybridized modes.

Figure 1.10: FDTD theory showing (A) the emergence of a second peak as a single nanocube $(90 \mathrm{~nm}$ diameter) approaches a dielectric substrate, and $(B, C)$ the field intensities for peaks 1 and 2 of the nanocube in contact with the substrate (the white line in the field pattern images represent the substrate).

Figure 1.11: Schematic representation of the lifetime of a plasmon resonance. Upon the plasmon's excitation (a) the excited electrons will form a thermal population of hot carriers, some of which can escape the nanoparticle as hot-electrons (b) others of which will undergo collisions within the metal lattice (c). These collisions will produce heat which will thermally dissipate through the nanoparticle (d).

Figure 1.12: Spectra of the Joule numbers for a large set of materials. Materials have been arranged in families: Plasmonic resonance out of the visible range (left), in the visible range (middle), and the singular case of silver (right) where data have been plotted considering two distinct data sets (the optical constants of Johnson and Christy, and of Palik's handbook).

Figure 1.13: Calculated Jo number plotted as a function of the resonance wavelength of ellipsoids made of various materials. 
Figure 1.14: Modified LaMer model describing the inclusion of an extra step for selecting nuclei with appropriated crystal structures.

Figure 1.15: Possible reaction paths in the polyol synthesis of Ag nanostructures. Initial nuclei formed after the reduction of Ag+ may fluctuate between different morphologies until growth to a critical size at which their morphology is locked into a single crystalline, singly twinned, or multiply twinned seed. The resultant respectively shaped seeds may grow into many different morphologies depending on choice of structure directing agent and presence of certain trace ions................................................................... 33

Figure 1.16: Mechanism of seed selection through oxidative etching.................................................. 34 Figure 1.17: a) Schematic drawing of the decahedron structure of multiple twinned seeds b) High resolution TEM of Ag multiple twinned seed showing c) distorted boundary region that results from structural strain.

Figure 1.18: Structure of Polyvinylpyrrolidone. Dashed red line indicates the bond between the two constituent components

Figure 1.19: Molecular structure of Polystyrene

Figure 1.20: Several general configurations of metal nanoparticle polymer composites discussed in this review, including nanoparticles (I) immobilized on a thin polymeric film adsorbed or spin-coated on a substrate, (II) three-dimensionally distributed within a polymer gel matrix, (III) adsorbed on polymer brush grafted-to or -from the substrate, (IV) infiltrated within the brush, (V) decorated on polymer fibers electrospun on the substrate and (VI) decorated on free-standing polymer fibers.

Figure 1.21: Photo of NIMA Langmuir-Schaefer Trough with Components Labelled.

Figure 1.22: Blodgett deposition (left) and Schaefer deposition (right). Reproduced from ( ${ }^{153}$ ). Copyright Elsevier 2006.

Figure 1.23: Scheme of Basic Object Layout in FDTD Model..

Figure 1.24: Illustration of the axes of polarization for the $0^{\circ}$ (blue arrow) and $90^{\circ}$ (red arrow) light used in FDTD calculations. The yellow star in the centre of the two AgNCs represents the injection of the light along the z-axis.

Figure 1.25: Schematic illustration of longitudinal (left) and transverse (right) coupling modes in an PVP capped AgNC dimer. The top image illustrates both coupling modes on the dimer simultaneously. ........ 51 Figure 3.1: Extinction Spectra of AgNC in Solution at Different Growth Times. ......................................60 Figure 3.2: A) Large Scale TEM image of AgNCS B) Medium Scale TEM image of AgNCs C) Small Scale TEM image of AgNCS D) Histogram of AgNC Edge Length Measurements taken with TEM Software.

Figure 3.3: Extinction Spectra of initial AgNC solution immediately after synthesis, and after 4 months and 1 year of being stored.

Figure 3.4: AFM images of A) a PS thin film prepared with a 3\% weight solution and B) a scratched PS thin film used for thickness measurements.

Figure 3.5: Left: Extinction spectrum of AgNC deposited on PS thin film. Right: $10 \mu \mathrm{m}$ x $10 \mu \mathrm{m}$ AFM image of the same deposited AgNC monolayer.

Figure 3.6: $10 \mu \mathrm{m} \times 10 \mu \mathrm{m}$ AFM images of AgNC monolayer after 30s, 90s, 160s (going left to right across the top row); 220s, 250s and 370s (going left to right across bottom row) of exposure to $458 \mathrm{~nm}$ laser at $17 \mathrm{~mW}$. An AFM images of the surface prior to laser exposure can be seen in Figure 6.10......

Figure 3.7: $10 \mu \mathrm{m} \times 10 \mu \mathrm{m}$ AFM images of AgNC monolayer before exposure to $568 \mathrm{~nm}$ laser at $65 \mathrm{~mW}$, after 30s, 60s (going left to right across top row), 90s, 120s and 180s (going left to right across bottom row). 
Figure 3.8: EEF of $30 \mathrm{~nm}, 45 \mathrm{~nm}$ and $60 \mathrm{~nm}$ SS dimers at $568 \mathrm{~nm}$ calculated with $0^{\circ}$ polarized light $(A-C)$ and $90^{\circ}$ polarized light (D-F). The $2 D \mathrm{Y}$ plane was used for the calculations with $0^{\circ}$ and $90^{\circ}$ polarized light. The coloured axis to the left of the images indicates the magnitude of the EEF. In D-F the dotted line indicates the junction between the individual cubes.

Figure 6.1: A) Large Scale TEM image of AgNC B) Medium Scale TEM image of AgNCs C) Small Scale TEM image of AgNC D) Histogram of AgNC Edge Length Measurement from TEM image set taken 4 months

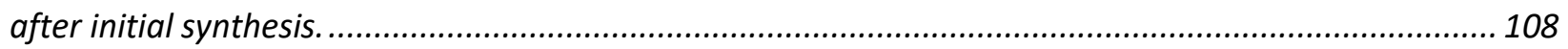

Figure 6.2: X-axis Cross section taken in Nova Software to measure thickness of PS thin Film............... 109 Figure 6.3: $10 \mu \mathrm{m} \times 10 \mu \mathrm{m}$ AFM of Deposited AgNC monolayer on PS with Nanorods.......................... 109 Figure 6.4: Extinction spectra (A) and $5 \mu m \times 5 \mu m$ AFM images of AgNC monolayers deposited at pressures of $0 \mathrm{mN} / \mathrm{m}(B), 1 \mathrm{mN} / \mathrm{m}(C), 2 \mathrm{mN} / \mathrm{m}(D), 3 \mathrm{mN} / \mathrm{m}(E)$ and $4 \mathrm{mN} / \mathrm{m}(F)$............................. 110 Figure 6.5: Extinction spectra (A) and $10 \mu \mathrm{m} \times 10 \mu \mathrm{m}$ AFMs of AgNC monolayers deposited at pressures of $2 \mathrm{mN} / \mathrm{m}(B), 4 \mathrm{mN} / \mathrm{m}(C), 6 \mathrm{mN} / \mathrm{m}(D), 8 \mathrm{mN} / \mathrm{m}(E)$.

Figure 6.6: AFM images of AgNC monolayer after being exposed to a $458 \mathrm{~nm}$ laser at $40 \mathrm{~mW}$ for 15 second ( $A$ ) and 5 seconds (B) and at $23 \mathrm{~mW}$ for 15 seconds (C) and 5 seconds (D). The diameters of the circular patterned regions were measured using Nova software to be approximately $18.24 \mu \mathrm{m}, 19.72 \mu \mathrm{m}$, $13.05 \mu \mathrm{m}$ and 12.67 respectively. 112 Figure 6.7: AFM images of AgNC monolayer after being exposed to a $458 \mathrm{~nm}$ laser at $17 \mathrm{~mW}$ for 5 minutes $(A)$ and 3 minutes $(C)$. $B$ and $D$ are zoomed in images of the regions outlined by the white square in $A$ and $C$ respectively. The diameter of patterned areas in $A$ and $C$ were measured with Nova to be $12.29 \mu \mathrm{m}(\mathrm{A})$ and $12.09 \mu \mathrm{m}$ (C).

Figure 6.8: AFM images of AgNC monolayer after being exposed to a $568 \mathrm{~nm}$ laser at $50 \mathrm{~mW}$ for 30 seconds (A), an additional 2 minutes (B) and an additional 5 minutes (C). Even after 7 minutes and 30 seconds of exposure at $50 \mathrm{~mW}$ there was no patterning observed. Figure 6.9: AFM images of AgNC monolayer after being exposed to a $568 \mathrm{~nm}$ laser at $65 \mathrm{~mW}$ for 2 minutes (A), 1 minute (B) and 20 seconds (C). D, E and $F$ are zoomed in images of the partially embedded regions in $A, B$ and $C$ respectively. The diameters of the circular patterned regions were measured using Nova software to be approximately $11.18 \mu \mathrm{m}(A), 11.13 \mu \mathrm{m}$ (B) and $8.26 \mu \mathrm{m}$ (C).

Figure 6.10: $10 \mu \mathrm{m} \times 10 \mu \mathrm{m}$ AFM of AgNC monolayer used for selective embedment experiments of individual cubes before laser exposure.

Figure 6.11: $10 \mu \mathrm{m} \times 10 \mu \mathrm{m}$ AFM of AgNC monolayer used for selective embedment experiments of individual cubes after 30 s of laser exposure.

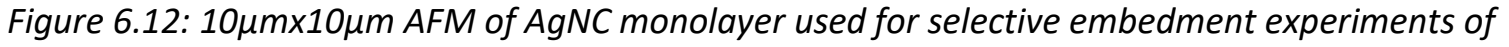
individual cubes after 60 s of laser exposure.

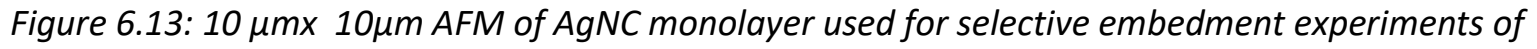
individual cubes after 90 s of laser exposure.

Figure 6.14: $10 \mu \mathrm{m} \times 10 \mu \mathrm{m}$ AFM of AgNC monolayer used for selective embedment experiments of individual cubes after 130 s of laser exposure.

Figure 6.15: $10 \mu \mathrm{m} \times 10 \mu \mathrm{m}$ AFM of AgNC monolayer used for selective embedment experiments of individual cubes after 160s of laser exposure.....

Figure 6.16: $10 \mu \mathrm{m} \times 10 \mu \mathrm{m}$ AFM of AgNC monolayer used for selective embedment experiments of individual cubes after 200s of laser exposure. 121 Figure 6.17: $10 \mu \mathrm{m} \times 10 \mu \mathrm{m}$ AFM of AgNC monolayer used for selective embedment experiments of individual cubes after 220 s of laser exposure...... 
Figure 6.18: $10 \mu \mathrm{m} \times 10 \mu \mathrm{m}$ AFM of AgNC monolayer used for selective embedment experiments of individual cubes after 250s of laser exposure.

Figure 6.19: $10 \mu \mathrm{m} \times 10 \mu \mathrm{m}$ AFM of AgNC monolayer used for selective embedment experiments of individual cubes after 370 s of laser exposure.

Figure 6.20: $10 \mu \mathrm{m} \times 10 \mu \mathrm{m}$ AFM of AgNC monolayer used for selective embedment experiments of individual cubes after 610s of laser exposure.....

Figure 6.21: $20 \mu \mathrm{m} \times 20 \mu \mathrm{m}$ AFM of AgNC monolayer after being exposed to $458 \mathrm{~nm}$ laser for 610s. The diameter of the circular pattern was measured with Nova to be $17.13 \mu \mathrm{m}$. 126

Figure 6.22: Outline of different regions of original AFM images used for zoomed-In images in Figures 6.23-6.25. The black, blue and yellow rectangles outline Region I, II and III respectively. The regions are shown here using the $10 \mu \mathrm{m} \times 10 \mu \mathrm{m}$ AFM taken 30s after laser exposure for reference.

Figure 6.23: Close-up of Region I following 30s, 60s, 90s (A-C), 130s, 160s, 200s (D-F), 220s, 250s, 370s

(G-I), and 610s (J) of exposure with the $458 \mathrm{~nm}$ laser.

Figure 6.24: Close-up of Region II following 30s, 60s, 90s, 130s, 160s (A-E), 200s, 220s, 250s, 370s and 610 s (F-J) of exposure with the $458 \mathrm{~nm}$ laser.

Figure 6.25: Close-up of Region III following 30s, 60s, 90s, 130s, 160s (A-E), 200s, 220s, 250s, 370s and $610 \mathrm{~s}$ (F-J) of exposure with the $458 \mathrm{~nm}$ laser.

Figure 6.26: Outline of different regions used for zoomed-In images in Figures 6.27-6.29. The blue, green and white rectangle outline Regions $A, B$ and $C$ respectively. The regions are shown here using the $10 \mu m$ $x 10 \mu m$ AFM taken before laser exposure for reference.

Figure 6.27: Close-up AFM images of Region $A$ before laser exposure (A), and after 30s (B), 60s (C), 90s (D), 120s (E) and 180s (F) of exposure to the $568 \mathrm{~nm}$ laser.

Figure 6.28: Close-up AFM images of Region B before laser exposure (A), and after 30s (B), 60s (C), 90s (D), $120 \mathrm{~s}(E)$ and $180 \mathrm{~s}(F)$ of exposure to the $568 \mathrm{~nm}$ laser.

Figure 6.29: Close-up AFM images of Region $C$ before laser exposure (A), and after 30s (B), 60s (C), $90 \mathrm{~s}$

(D), $120 \mathrm{~s}$ (E) and 180s (F) of exposure to the $568 \mathrm{~nm}$ laser.....

Figure 6.30: Cross sections used to determine the background height in Region A for AFM images taken 60 s after laser exposure. These five cross section measurements were replicated in Region A for the AFM images taken before laser exposure and after each of the laser exposure trials. The lowest height in each cross section was considered the lowest value in the cross section with minimal noise.

Figure 6.31: Cross sections used to determine the background height in Region B for AFM images taken 60 s after laser exposure. These five cross section measurements were replicated in Region $B$ for the AFM images taken before laser exposure and after each of the laser exposure trials. The lowest height in each cross section was considered the lowest value in the cross section with minimal noise. 134 Figure 6.32: Cross sections used to determine the background height in Region C for AFM images taken 60 s after laser exposure. These five cross section measurements were replicated in Region C for the AFM images taken before laser exposure and after each of the laser exposure trials. The lowest height in each cross section was considered the lowest value in the cross section with minimal noise.

Figure 6.33: Close up of Region A used for cube height measurements. The cubes whose heights were measured are numbered. An asterix next to the number indicates the cubes that are considered to be in clusters. DS Dimers and SS Dimers are circled and identified by solid and dotted lines respectively. The numbering is shown here on the AFM taken before laser exposure for reference. 136 Figure 6.34: Close up of Region B used for cube height measurements. The cubes whose heights were measured are numbered. An asterix next to the number indicates the cubes that are considered to be in 
clusters. Row Trimers and Triangular Trimers are circled and identified by solid and dotted lines respectively. Cubes 21 and 22 are considered to be in a SS Dimer and cubes 23 and 24 are considered to be in a DS Dimer. The numbering is shown here on the AFM taken before laser exposure for reference.

Figure 6.35: Close up of Region C used for cube height measurements. The cubes whose heights were measured are numbered and lettered. In region $C$ the cubes are classified by size: the cubes with white numbers, black letters, and black numbers with grey highlight and are classified as small, medium and large respectively. For these cubes an asterix indicates that they are in a small cluster (4 cubes or less). The cubes with red numbers are cubes in clusters with $5+$ cubes. The non-underlined red numbers indicate cubes in large cluster I and the underlined red numbers indicate cubes in large cluster II. The numbering is shown here on the AFM taken before laser exposure for reference................................ 139 Figure 6.36: FDTD Calculated Absorption Spectra for AgNC of Different sizes...................................... 142 Figure 6.37: FDTD Calculated Absorption Spectra for SS Dimers of AgNC. The numbers in brackets in the legend indicate the angle of polarization.

Figure 6.38: FDTD Calculated Absorption Spectra for DS Dimers of AgNC. The numbers in brackets in the legend indicate the angle of polarization.

Figure 6.39: FDTD Calculated Absorption Spectra for Row Trimers of AgNC of Different sizes. The numbers in brackets in the legend indicate the angle of polarization.

Figure 6.40: FDTD Calculated Absorption Spectra for Triangle Trimers of AgNC of Different sizes. The numbers in brackets in the legend indicate the angle of polarization.

Figure 6.41: FDTD Calculated Absorption Spectra for Diamond Tetramers of AgNC of Different sizes. The numbers in brackets in the legend indicate the angle of polarization. Figure 6.42: 2D plans used to calculate the EEFs for the dimers of AgNCs where nanocubes are the same size. The planes are shown on the $x y(A), x z(B)$ and $y z(C)$ axis...... 145 Figure 6.43: $2 D$ planes used to calculate the EEFs for the dimers of AgNCs where nanocubes are the different sizes. The planes are shown on the $x y(A), x z(B)$ and $y z(C)$ axis. 146 Figure 6.44: $2 D$ planes used to calculate the EEFs for the row trimers of AgNCs. The planes are shown on the $x y(A), x z(B)$ and $y z(C)$ axis.

Figure 6.45: 2D planes used to calculate the EEFs for the triangle trimers of AgNCs. The planes are shown on the $x y(A), x z(B)$ and $y z(C)$ axis. 146 Figure 6.46: $2 D$ planes used to calculate EEF for the diamond tetramers of AgNCs. The planes are shown on the $x y(A), x z(B)$ and $y z(C)$ axis.

Figure 6.47: EEFs of $30 \mathrm{~nm} / 45 \mathrm{~nm}, 30 \mathrm{~nm} / 60 \mathrm{~nm}$ and $45 \mathrm{~nm} / 60 \mathrm{~nm}$ DS dimers at $568 \mathrm{~nm}$ calculated with $0^{\circ}$ polarized Light $(A-C)$ and $90^{\circ}$ polarized light (D-F). A $2 D$ Y plane was used for the calculations with $0^{\circ}$ and $90^{\circ}$ polarized Light. The coloured axis to the left of the images indicates the magnitude of the EEF.

Figure 6.48: EEFs of row trimers with $30 \mathrm{~nm}, 45 \mathrm{~nm}$ and $60 \mathrm{~nm}$ cubes at $568 \mathrm{~nm}$ calculated with $0^{\circ}$ polarized light $(A-C)$ and $90^{\circ}$ polarized light (D-F). A $2 D$ Y plane was used for the calculations with $0^{\circ}$ polarized light and $90^{\circ}$ polarized light. The coloured axis to the left of the images indicates the magnitude of the EEF.

Figure 6.49: EEF of $30 \mathrm{~nm}$ triangle trimer at $568 \mathrm{~nm}$. A-C show the respective $Z, X-R$ and $Y$ planes calculated with $0^{\circ}$ polarized light and $D$ and $E$ show the $Z$ and $X-R$ planes calculated with $90^{\circ}$ polarized light. The coloured axis to the left of the images indicates the magnitude of the EEF. 
Figure 6.50: EEF of $45 \mathrm{~nm}$ triangle trimer at $568 \mathrm{~nm}$. A-C show the respective $Z, X-R$ and $Y$ planes calculate with $0^{\circ}$ Polarized Light and $D-E$ show the $Z, X-R$ and $Y$ planes calculated with $90^{\circ}$ Polarized Light. The coloured axis to the left of the images indicates the magnitude of the EEF.

Figure 6.51: EEF of $60 \mathrm{~nm}$ triangle trimer at $568 \mathrm{~nm}$. A-C show the respective $Z, X-R$ and $Y$ planes calculate with $0^{\circ}$ polarized light and $D$ and $E$ show the $Z$ and $X-R$ planes calculated with $90^{\circ}$ polarized light. The coloured axis to the left of the images indicates the magnitude of the EEF.

Figure 6.52: EEF of $30 \mathrm{~nm}$ AgNC diamond tetramer at $568 \mathrm{~nm}$. A-C show the respective $Z, X-R$ and $Y$ planes calculate with $0^{\circ}$ polarized light and $D-F$ show the $Z, X-R$ and $X-M$ planes calculated with $90^{\circ}$ polarized light. The coloured axis to the left of the images indicates the magnitude of the EEF.

Figure 6.53: EEF of $45 \mathrm{~nm}$ AgNC diamond tetramer. A-C show the respective $Z, X-R$ and $Y$ planes calculate with $0^{\circ}$ polarized light and $D-F$ show the $Z, X-R$ and $X-M$ planes calculated with $90^{\circ}$ polarized light. The coloured axis to the left of the images indicates the magnitude of the EEF...... 150 Figure 6.54: EEF of $60 \mathrm{~nm}$ AgNC diamond tetramer. A-C show the respective $Z, X-R$ and $Y$ planes calculate with $0^{\circ}$ polarized light and $D-F$ show the $Z, X-R$ and $X-M$ planes calculated with $90^{\circ}$ polarized light. The coloured axis to the left of the images indicates the magnitude of the EEF...... 150 Figure 6.55: EEFs of $30 \mathrm{~nm}, 45 \mathrm{~nm}$ and $60 \mathrm{~nm}$ SS dimers calculated with $0^{\circ}$ polarized light $(A-C)$ and $90^{\circ}$ polarized light (D-F). A $2 D$ Y plane was used for the calculations with $0^{\circ}$ polarized light and $90^{\circ}$ polarized light. The coloured axis to the left of the images indicates the magnitude of the EEF. The EEFs were calculated at $520 \mathrm{~nm}(A), 566 \mathrm{~nm}(B), 674 \mathrm{~nm}(C), 444 \mathrm{~nm}(D), 446 \mathrm{~nm}$ (E) and $398 \mathrm{~nm}$ (F). 151 Figure 6.56: EEFs of $30 \mathrm{~nm} / 45 \mathrm{~nm}, 30 \mathrm{~nm} / 60 \mathrm{~nm}$ and $45 \mathrm{~nm} / 60 \mathrm{~nm}$ DS dimers calculated with $0^{\circ}$ polarized light $(A-C)$ and $90^{\circ}$ polarized light (D-F). A $2 D$ Y plane was used for the calculations with $0^{\circ}$ and $90^{\circ}$ polarized light. The coloured axis to the left of the images indicates the magnitude of the $E E F$. The EEFs were calculated at $558 \mathrm{~nm}(A), 576 \mathrm{~nm}(B), 622 \mathrm{~nm}(C), 404 \mathrm{~nm}$ (D), $404 \mathrm{~nm}$ (E) and $400 \mathrm{~nm}$ (F). . 152 Figure 6.57: EEFs of $30 \mathrm{~nm}, 45 \mathrm{~nm}$ and $60 \mathrm{~nm}$ row trimers calculated with $0^{\circ}$ polarized light $(A-C)$ and $90^{\circ}$ polarized light (D-F). A $2 D$ Y plane was used for the calculations with $0^{\circ}$ polarized light and $90^{\circ}$ polarized light. The coloured axis to the left of the images indicates the magnitude of the EEF. The EEFs were calculated at $600 \mathrm{~nm}(A), 688 \mathrm{~nm}(B), 660 \mathrm{~nm}(C), 418 \mathrm{~nm}(D), 444 \mathrm{~nm}$ (E) and $372 \mathrm{~nm}$ (F)................. 152 Figure 6.58: $E E F$ of $30 \mathrm{~nm}$ triangle trimer. $A-C$ show the respective $Z, X-R$ and $Y$ planes calculated with $0^{\circ}$ polarized light at $514 \mathrm{~nm}$ and $D-F$ show the $Z, X-R$ and planes calculated with $90^{\circ}$ polarized light at 538 $\mathrm{nm}$. The coloured axis to the left of the images indicates the magnitude of the EEF. 153 Figure 6.59: EEF of $45 \mathrm{~nm}$ triangle trimer. A-C show the respective $Z, X-R$ and $Y$ planes calculated with $0^{\circ}$ polarized light at $558 \mathrm{~nm}$ and $D-F$ show the $Z, X-R$ and planes calculated with $90^{\circ}$ polarized light at 594 $\mathrm{nm}$. The coloured axis to the left of the images indicates the magnitude of the EEF..... Figure 6.60: EEF of $60 \mathrm{~nm}$ triangle trimer. A-C show the respective $Z, X-R$ and $Y$ planes calculated with $0^{\circ}$ polarized light at $596 \mathrm{~nm}$ and $D-F$ show the $Z, X-R$ and planes calculated with $90^{\circ}$ polarized light at 626 $\mathrm{nm}$. The coloured axis to the left of the images indicates the magnitude of the EEF. 154 Figure 6.61: EEF of $30 \mathrm{~nm}$ diamond tetramer. A-C show the respective $Z, X-R$ and $Y$ planes calculated with $O^{\circ}$ polarized light at $502 \mathrm{~nm}$ and $D-F$ show the $Z, X-R$ and planes calculated with $90^{\circ}$ polarized light at $570 \mathrm{~nm}$. The coloured axis to the left of the images indicates the magnitude of the EEF....................... 154 Figure 6.62: EEF of $45 \mathrm{~nm}$ diamond tetramer. A-C show the respective $Z, X-R$ and $Y$ planes calculated with $0^{\circ}$ polarized light at $544 \mathrm{~nm}$ and $D-F$ show the $Z, X-R$ and planes calculated with $90^{\circ}$ polarized light at $650 \mathrm{~nm}$. The coloured axis to the left of the images indicates the magnitude of the EEF. 155 
Figure 6.63: $E E F$ of $60 \mathrm{~nm}$ diamond tetramer. A-C show the respective $Z, X-R$ and $Y$ planes calculated with $0^{\circ}$ polarized light at $558 \mathrm{~nm}$ and $D-F$ show the $Z, X-R$ and planes calculated with $90^{\circ}$ polarized light at $740 \mathrm{~nm}$. The coloured axis to the left of the images indicates the magnitude of the EEF...................... 155 


\section{List of Tables}

Table 3.1: Average Change in Height After Initial 30s Laser Exposure ( $\left.\Delta h_{0-30 s}\right)$ and after Total 3 min of Exposure $\left(\Delta h_{0-180 s}\right)$ for Cubes in Region A ....................................................................................... 77 Table 3.2: Average Change in Height After Initial 30s Laser Exposure ( $\left.\Delta h_{0-30 s}\right)$ and after Total 3 min of Exposure ( $\left.\Delta h_{0-180 s}\right)$ for Cubes in Region $B$ .77

Table 3.3: Average Change in Height After Initial 30s Laser Exposure ( $\left.\Delta h_{0-30 \mathrm{~s}}\right)$ and after Total $3 \mathrm{~min}$ of Exposure $\left(\Delta h_{0-180 s}\right)$ for Different Sized Cubes in Region $C$.................................................................. 77

Table 3.4: Average Change in Height After Initial 30s Laser Exposure ( $\left.\Delta h_{0-30 s}\right)$ and after Total $3 \mathrm{~min}$ of Exposure $\left(\Delta h_{0-180 s}\right)$ of Cubes Large Cluster in Region C ...................................................................... 78 Table 3.5: FDTD Calculated Absorption Cross Section of Different AgNCs and AgNC Clusters at $568 \mathrm{~nm}$ using $0^{\circ}$ and $90^{\circ}$ Polarized Light . .82

Table 3.6: FDTD Calculated Peak Absorption Wavelength for Different Types of AgNC Clusters.............. 91

Table 6.1: Background Height in Region A of AFM Images Before and After Laser Exposure Trials ........ 133

Table 6.2: Background Height in Region B of AFM Images Before and After Laser Exposure Trials ........ 134

Table 6.3: Background Height in Region C of AFM Images Before and After Laser Exposure Trials ........ 135 Table 6.4: Corrected Height of AgNCs Before Laser Exposure and After Laser Exposure Intervals in Region

A. . 136

Table 6.5: Corrected Height of AgNCs Before Laser Exposure and After Laser Exposure Intervals in Region B. 137

Table 6.6: Corrected Height of AgNCs Before Laser Exposure and After Laser Exposure Intervals for Small Cubes in Region $C$...... 139

Table 6.7: Corrected Height of AgNCs Before Laser Exposure and After Laser Exposure Intervals for Medium Cubes in Region $C$. 140

Table 6.8: Corrected Height of AgNCs Before Laser Exposure and After Laser Exposure Intervals for Large Cubes in Region $C$... Table 6.9: Corrected Height of AgNCs Before Laser Exposure and After Laser Exposure Intervals for Cubes in Large Clusters Region $C$. 141

Table 6.10: Comparison of Average Change in Height After Initial 30s Laser Exposure ( $\Delta h_{0-30 s}$ ) and after Total 3min of Exposure ( $\left.\Delta h_{0-180 s}\right)$ of Different Types of Cube Clusters in the Three Different Regions...... 141 


\section{Introduction}

Over the past decades there has been a dramatic increase in the application of nanotechnology to solve problems in biotechnology, medicine, engineering, and device technology. The unique optical and electronic properties of nanoparticles have been harnessed for use in biosensors, immunoassays, optical imaging and spectroscopy techniques, catalysis, electronic and photovoltaic devices, and nanopatterning. ${ }^{1,2}$ As the demand for nanotechnology solutions increases, so does the need for the development of novel nanomaterials and techniques to suit niche applications. This work describes the development of a novel technique to achieve nanopatterning via selective thermoplasmonic excitation of supported silver nanocrystals.

In order to understand the theoretical principles behind these experiments, and how they may contribute to existing knowledge, an understanding of plasmonics and nanoparticle/polymer composites is required. The following section will provide an overview of plasmonics, focusing on the plasmonic properties of nanoparticles, the variables that affect them, and how they can be harnessed for localized heating applications. Since the plasmonic properties of nanoparticles are so dependent on morphology and local environment, the shape-controlled synthesis of colloidal nanocrystals and the use of polymer thin-film support will also be discussed. This section will conclude with a discussion of numerical modelling techniques, focusing on the simulation software used in this study to elucidate the experimental results and provide direction for future experiments.

\subsection{Plasmonics}

A plasmon is a quantum (smallest unit of discrete energy) of plasma oscillations. ${ }^{3}$ Also referred to as Langmuir Waves, plasma oscillations are the organized rapid motion of free electrons in the conduction band of a conductive material. ${ }^{4}$ A plasmon oscillates at a defined frequency and will only oscillate when excited by incoming electromagnetic (EM) radiation of the same frequency. ${ }^{3,5,6}$ This phenomenon is 
known as plasmon resonance (PR). Plasmonics is the field of science that investigates the interactions between EM radiation and plasmon electrons that lead to plasmon resonance. ${ }^{7,8}$

While seemingly complex, plasmon oscillations can be described using classical physics. ${ }^{3}$ The various types of plasmons that can exist in different materials, the equations describing their behaviour, and the things that affect their resonance behaviours will be described in the sections below. Though plasmon resonance has been shown to occur in other conducting materials, for the sake of simplicity it will be described in this introduction in the context of occurring in metals. ${ }^{9}$

\subsubsection{Bulk, Surface and Localized Plasmons}

There are three different types of plasmons that can exist in different types of metallic materials: bulk plasmons, surface plasmons, and localized surface plasmons. For a bulk metal of infinite size, the frequency of oscillations of electrons in a plasmon $\left(\omega_{p}\right)$ can be described with the following equation

$$
\omega_{\mathrm{p}}=\sqrt{\frac{\mathrm{Ne}^{2}}{\varepsilon_{0} \mathrm{~m}_{e}}}
$$

where $\mathrm{N}$ is the number density of conduction electrons, $\varepsilon_{0}$ is the dielectric constant of vacuum, e is the charge of an electron, and $\mathrm{m}_{\mathrm{e}}$ is the effective mass of an electron. ${ }^{5}$ The energy of a bulk plasmon $\left(E_{p}\right)$ can be described by Equation 2 in which $\varepsilon_{0}$ is the permittivity of free space, $\mathrm{N}$ is the electron number density, $\mathrm{e}$ is the electron charge, and $\mathrm{m}_{\mathrm{e}}$ is the electron mass. ${ }^{1,5}$

$$
E_{p}=\hbar \sqrt{\frac{\mathrm{Ne}^{2}}{\varepsilon_{0} \mathrm{~m}_{e}}}
$$

In Equation 1 all the variables aside from $\mathrm{N}$ are constant. This illustrates that the bulk plasmon frequency of a select metal is dependent solely on its density of free electrons. ${ }^{5}$ Because free electron density depends on the identity of a metal, different metals will have different plasmon frequencies. The plasmon frequencies of most metals are within the ultraviolet (UV) region (corresponding to light with $\lambda$ of 100- 
$400 \mathrm{~nm})$ but the plasmon frequencies of alkali metals and noble metals $(\mathrm{Cu}, \mathrm{Ag}$, and $\mathrm{Au})$ are within the visible region (corresponding to light with $\lambda$ of $350-700 \mathrm{~nm}$ ). ${ }^{5,6}$

Electromagnetic radiation can rarely penetrate more than $50 \mathrm{~nm}$ deep in a metal surface. ${ }^{5}$ Because of this, bulk plasmons are of far less interest than their surface counter parts. There are two main types of surface plasmons: propagating surface plasmons (PSPs) and localized surface plasmons (LSPs).

PSPs occur when plasmons on the surface of a metal are excited and begin to oscillate along the interface of the metal surface and the dielectric medium (the non-conductive medium the metal surface is in). These plasmons can propagate in the $\mathrm{x}$ and $\mathrm{y}$ direction along the metal-dielectric interface for distances up to 100 s of $\mu \mathrm{m} .{ }^{8}$ As they move along the surface of a material, the amplitude of the oscillation decays exponentially until the energy dissipates. ${ }^{8}$ The oscillation of electrons around fixed ion cores that occurs in PSPs leads to areas of high and low electron density across the surface of a material. ${ }^{3,5,8}$ These areas of high and low electron density cause alternating areas of respective negative and positive charges along the surface of the metal. This phenomenon can be seen in Figure 1.1 below where the red lines in the figure indicate the electromagnetic field component of light that induces the oscillation of the electrons.

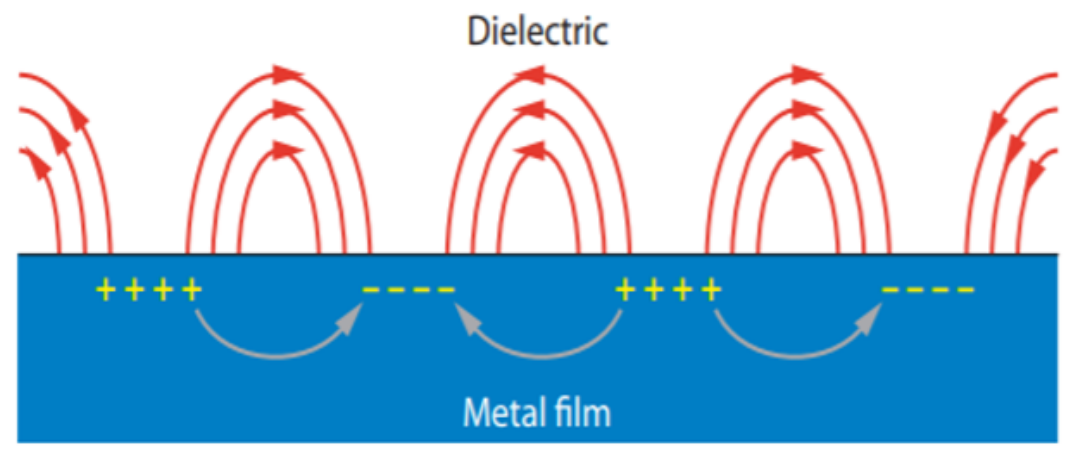

Figure 1.1: Schematic of Surface Plasmon resonance. Adapted with permission from (5). Copyright Annual Reviews of Physical Chemistry 2009. 
The frequency of a propagating surface plasmon $\left(\omega_{\mathrm{psp}}\right)$ can be theoretically related to the frequency of a bulk plasmon with Equation 3 below which assumes a metal-vacuum interface. ${ }^{5}$

$$
\omega_{\mathrm{psp}}=\omega_{\mathrm{p}} / \sqrt{2}
$$

While the wavelength used to excite bulk plasmons is always consistent for a given metal, the wavelength that corresponds to a surface plasmon resonance can vary because the oscillation frequency of a surface plasmon is dependent on both the refractive index of the metal and the dielectric medium. ${ }^{1,8,10}$ This makes surface plasmons very sensitive to changes in the local environment and it is a property that is exploited in many spectroscopy and sensing applications. ${ }^{4-8}$

When a surface plasmon is excited on a metal structure that is smaller than the wavelength of incident light used for excitation, the surface plasmon cannot propagate in an $\mathrm{x}$ or $\mathrm{y}$ direction. The plasmon instead is confined or "localized" to oscillating around the particle in what is known as localized surface plasmon resonance (LSPR). ${ }^{3,5,8}$ LSPR most commonly occurs in metal nanoparticles-metal crystals with all dimensions on the nanoscale.

When a metal nanoparticle's LSPR is excited, the electric field component of the light causes the free electrons in the conduction band of the metal to collectively move away from the centre of the mass of the particle towards the boundary. ${ }^{6}$ These free electrons moving away from the particle in one direction create significant charge separation generating dipoles within the particle. ${ }^{6}$ The strongest dipole is formed when the frequency of the incident light is the same as that of the plasmon leading to maximum constructive interference. Figure 1.2 illustrates dipole generation within a metal nanosphere via electron oscillations. 


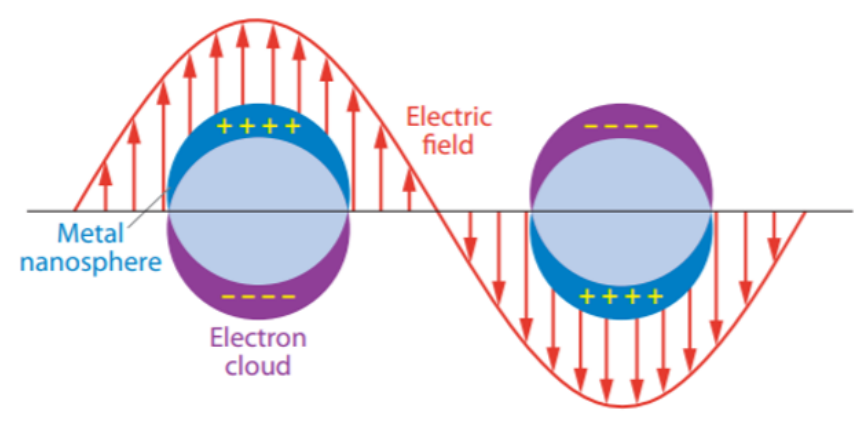

Figure 1.2: Schematic of nanoparticle dipole generated by Localized Surface Plasmon resonance. Adapted with permission from (5). Copyright Annual Reviews of Physical Chemistry 2009.

The presence of an LSP leads to an enhanced electric field near the particle's surface, which is strongest at the surface of the particle and decays exponentially with distance. ${ }^{3}$ The enhanced electric field caused by LSPR is referred to as a particle's near-field and it is higher in energy than the incident EM radiation used to induce LSPR oscillation. ${ }^{3}$

There are many different variables that affect the frequency of localized plasmons and their corresponding resonance. To explain LSPR physicists and chemists use Mie theory, a series of mathematical solutions that describe how particles interact with light.

\subsubsection{Mie Theory}

When any electromagnetic radiation such as light encounters an object there are two different phenomena that occur: absorption and scattering. In absorption, the incident electromagnetic radiation transfers energy to the object; once transferred, this energy will manifest in a different form in the object. ${ }^{11}$ In scattering the incoming electromagnetic radiation is absorbed by the object and re-emitted in a different direction. ${ }^{11}$ Extinction is the measure of any light that is not transmitted through a material and is the sum of absorption and scattering. ${ }^{11}$ 
Mie theory is a general solution to Maxwell's equations developed by Gustave Mie to describe the scattering of electromagnetic waves by a spherical particle..$^{6,12-14}$ It can be applied to metal nanoparticles, making the assumption that the particles are perfectly spherical. ${ }^{6}$ Values typically calculated with Mie theory include optical properties such as the diffusion efficiencies of absorption, scattering and extinction $\left(Q_{a b s,} Q_{a b s}\right.$ and $Q_{e x t}$ respectively) and their corresponding cross sections. ${ }^{13-15}$ Calculating these values is of interest to chemists and physicists as it allows them to explain and investigate optical properties of nanoparticles, such as the frequencies of light for which LSPR will occur..$^{12,13,15,16}$ The extinction cross section $\left(\sigma_{\text {ext }}\right)$, scattering cross section $\left(\sigma_{\text {sca }}\right)$, and absorption cross section $\left(\sigma_{\text {abs }}\right)$ of a spherical conducting particle embedded in a medium with a dielectric function of $\varepsilon_{\mathrm{m}}$ are described by the following equations

$$
\begin{gathered}
\sigma_{\text {ext }}=\frac{2 \pi}{|k|^{2}} \sum_{L=1}^{\infty}(2 L+1)\left[\operatorname{Re}\left(a_{L}+b_{L}\right)\right] \\
\sigma_{s c a}=\frac{2 \pi}{|k|^{2}} \sum_{L=1}^{\infty}(2 L+1)\left(\left|a_{L}\right|^{2}+\left|b_{L}\right|^{2}\right) \\
\sigma_{a b s}=\sigma_{\text {ext }}-\sigma_{s c a}
\end{gathered}
$$

where $k$ is the incoming wave-vector and it is equal to $\frac{2 \pi \sqrt{\varepsilon_{m}}}{\lambda}$ ( $\lambda$ is the wavelength of incident wave); $R$ is the radius of the particle; $e$ is the electron charge; and $\mathrm{L}$ is an integer representing the dipole $(\mathrm{L}=1)$, quadrupole $(\mathrm{L}=2)$, and higher modes of excitations $(\mathrm{L}=3,4, \ldots){ }^{3,12}$ Both $a_{L}$ and $b_{L}$ are scattering parameters composed of the Riccati-Bessel functions $\varphi_{L}$ and $\chi_{L}$ and are modelled by the equations

$$
\begin{aligned}
& a_{L}(R, \lambda)=\frac{m \varphi_{L}(m x)+\varphi_{L}^{\prime}(x)-\varphi_{L}^{\prime}(m x) \varphi_{L}(x)}{m \varphi_{L}(m x)+\chi_{L}^{\prime}(x)-\varphi_{L}^{\prime}(m x) \chi_{L}(x)} \\
& b_{L}(R, \lambda)=\frac{\varphi_{L}(m x)+\varphi_{L}^{\prime}(x)-m \varphi_{L}^{\prime}(m x) \varphi_{L}(x)}{\varphi_{L}(m x)+\chi_{L}^{\prime}(x)-m \varphi_{L}^{\prime}(m x) \chi_{L}(x)}
\end{aligned}
$$

where $m=\tilde{n} / n_{m}, \tilde{n}=n_{R}+i n_{I}$ is the complex refractive index of the metal, $n_{m}$ is the real refractive index of the surrounding medium, and $x=k_{m} r$, where $r$ is the radius of the particle. ${ }^{3,12}$ It is important to 
note that $k_{m}=2 \pi / \lambda_{m}$ and is defined as the wavenumber in the medium rather than the vacuum wavenumber. $^{1}$

In order to use Mie theory to provide insight to LSPR phenomena, Equations 4-8 need to be simplified. ${ }^{3}$ To simplify these equations the Rayleigh approximation is used. The Rayleigh approximation assumes that the nanoparticle is very small compared to the wavelength of the incoming electromagnetic radiation, and it satisfies the condition that the value of $x<<1$ for Equations 7 and 8.,12,13 In the Rayleigh regime of scattering, also referred to as the dipole regime or quasi static regime of scattering, the Riccati-Bessel functions can be simplified into the following: $6,3,12$

$$
\begin{aligned}
& a_{I} \approx-\frac{i 2 x^{3}}{3} \frac{m^{2}-1}{m^{2}+2} \\
& b_{I} \approx 0
\end{aligned}
$$

By considering the terms up to $x^{3}$ for approximation, $a_{I}$ and $b_{I}$ of higher order would be zero, and only the dipole resonance condition $(\mathrm{L}=1)$ is considered. ${ }^{3,12}$

In order to better express the extinction cross section one has to find the real part of $a_{I}$, which can be done by substituting $m=\left(n_{R}+i n_{I}\right) / n_{m}$ into Equation 9 to get Equation $11 .^{3,12}$

$$
a_{I} \approx-\mathrm{i} \frac{2 x^{3}}{3} \frac{n_{R}^{2}-n_{I}^{2}+i 2 n_{R} n_{I}-n_{m}^{2}}{n_{R}^{2}-n_{I}^{2}+i 2 n_{R} n_{I}-2 n_{m}^{2}}
$$

Then, to express $a_{I}$ in terms of dielectric functions instead of refractive indices, Equations $12-14$ can be used

$$
\begin{gathered}
\varepsilon_{1}=n_{R}^{2}-n_{I}^{2} \\
\varepsilon_{2}=2 n_{R} n_{I} \\
\varepsilon_{m}=n_{m}^{2}
\end{gathered}
$$


where $\varepsilon_{1}$ and $\varepsilon_{2}$ compose the real and imaginary components of a metal's complex dielectric function and $\varepsilon_{m}$ is the dielectric function of the material.,12 The real and imaginary components of $\varepsilon_{m}$ are also commonly represented as $\varepsilon_{r}$ and $\varepsilon_{i}$ and the two notations can be used interchangeably.

Substituting Equations 12-14 into Equation 11 and using the resulting expression for $a_{I}$ in Equations 4 and 5 yields new expressions for the extinction and scattering cross sections. ${ }^{3,12}$

$$
\begin{gathered}
\sigma_{\text {ext }}=\frac{18 \pi \varepsilon_{m}^{2 / 3} V}{\lambda} \frac{\varepsilon_{2}(\lambda)}{\left[\varepsilon_{1}(\lambda)+2 \varepsilon_{m}\right]^{2}+\varepsilon_{2} \lambda^{2}} \\
\sigma_{s c a}=\frac{34 \pi^{4} \varepsilon_{m}^{2} V^{2}}{\lambda^{4}} \frac{\left[\varepsilon_{1}(\lambda)+\varepsilon_{m}\right]^{2}+\varepsilon_{2} \lambda^{2}}{\left[\varepsilon_{1}(\lambda)+2 \varepsilon_{m}\right]^{2}+\varepsilon_{2} \lambda^{2}}
\end{gathered}
$$

As a nanoparticle's optical extinction has a maximum at the nanoparticles plasmon resonant frequency, the extinction cross section is of extreme interest to those who work with nanomaterials. ${ }^{3,6}$ Based on Equation 6 it can be seen that extinction is the sum of the absorption and scattering cross sections. However, within the Rayleigh regime the main contribution to the extinction is the absorption and scattering is negligible. ${ }^{6,17}$ Because of this, the individual contributions of the absorption and scattering cross sections to the extinction cross sections are rarely calculated. Instead scientists use the extinction cross section to investigate under what conditions LSPR will occur. Equation 15 expresses the extinction cross section in terms of the volume (V) of a spherical nanoparticle. Another common form of the extinction cross section equation that uses the particles radius (R) can be seen in Equation 17,

$$
\sigma_{e x t}=\frac{24 \pi^{2} \varepsilon_{m}^{3 / 2} R^{3}}{\lambda} \frac{\varepsilon_{2}}{\left[\varepsilon_{1}+2 \varepsilon_{m}\right]^{2}+\varepsilon_{2}^{2}}
$$

where $\varepsilon_{m}$ is the dielectric constant of the medium surrounding the particle and $\varepsilon_{1}$ and $\varepsilon_{2}$ are the real and imaginary parts of the metal's dielectric constant $\left(\varepsilon_{m}\right)$ respectively. ${ }^{6}$ In Equations 15 and 16 the component of the metal's dielectric constant are expressed as functions of lambda. The $(\lambda)$ were removed 
for Equation 17 for the sake of simplicity but it is important to note that both $\varepsilon_{1}$ and $\varepsilon_{2}$ will change with the wavelength of the incoming electromagnetic radiation. ${ }^{18,19}$

The resonant condition of a nanoparticle will occur when it's $\sigma_{\text {ext }}$ value is at a maximum. ${ }^{3}$ This happens when the bracketed expression in the denominator of Equation 17 approaches zero. When $\left[\varepsilon_{1}+2 \varepsilon_{m}\right]^{2}$ approaches zero, $\sigma_{\text {ext }}$ will become very large and the extinction at this particular frequency will become extremely strong. ${ }^{3,6,19}$ These conditions are known as a resonance condition and need to be met in order for a material to support plasmon resonance. ${ }^{3,6,19}$

\subsubsection{Effect of Chemical Composition on LSPR}

A material can only meet the resonance condition if the value of $\varepsilon_{1}$ is close to $-2 \varepsilon_{m},\left(\varepsilon_{1} \approx-2 \varepsilon_{m}\right)$. This is most readily achieved when the value of $\varepsilon_{1}$ is negative and the value of $\varepsilon_{2}$ is a small positive number. ${ }^{8}$ The majority of standard dielectric elements and compounds have $\varepsilon_{1}$ values of 1-50, however, alkali metals (such as $\mathrm{Na}, \mathrm{K}$ and $\mathrm{Li}$ ) and transition metals (such as $\mathrm{Al}, \mathrm{Cu}$ and $\mathrm{Au}$ ) possess the electronic properties required to support plasmon resonance in the visible and near infrared regions. ${ }^{6-9,12,13,19-21}$

When considering a plasmonic material it is important to establish that the material not only meets the resonance condition but also possesses a strong plasmon. A strong plasmon, also referred to as an intense plasmon, is one that has a sharp, well-defined resonance frequency and will exhibit minimal damping (decreased amplitude of oscillation over time)..$^{9,19,22}$ The intensity of a plasmon is measured by the quality factor, $Q$, which is given by the equation

$$
Q=\frac{\omega\left(\frac{\mathrm{d} \varepsilon_{1}}{\mathrm{~d} \omega}\right)}{2\left(\varepsilon_{2}\right)^{2}}
$$


where $\omega$ is the angular frequency of light $\left(\frac{2 \pi}{\lambda}\right)$ and $\varepsilon_{1}$ and $\varepsilon_{2}$ are the real and imaginary parts of the dielectric function respectively., ${ }^{9,19,22}$ In general, in order for a material to be used for a specific plasmonic application its $Q$ value should be greater than $10 .^{9,19,22}$

$\mathrm{Ag}$ and $\mathrm{Au}$ are generally the materials of choice for plasmonic applications as they posses optimal optical and chemical characteristics. As can be seen in Figure 1.3, $\mathrm{Ag}$ has a negative $\varepsilon_{1}$ for wavelengths between $\sim 320 \mathrm{~nm}-800 \mathrm{~nm}$ and a small positive value of $\varepsilon_{2}$ between $300 \mathrm{~nm}-800 \mathrm{~nm}$; and Au has a negative $\varepsilon_{1}$ from $\sim 480 \mathrm{~nm}-800 \mathrm{~nm}$ and a small positive value of $\varepsilon_{2}$ between $550-750 \mathrm{~nm} \cdot{ }^{19}$ This means that their plasmon resonances are within the visible region of the spectrum and can be excited by standard optical sources and methods. ${ }^{23}$ However, the value of $\varepsilon_{2}$ for gold is high for wavelengths less than $450 \mathrm{~nm}$, shows a broad transition between $450-650 \mathrm{~nm}$, and begins increasing again past $750 \mathrm{~nm}$, resulting in Au having broader resonances and lower extinction efficiency than $\mathrm{Ag}$ over the visible wavelength range. Ag also has a consistently higher quality factor than gold for most wavelengths, as can be seen in Figure 1.4. ${ }^{19,24}$
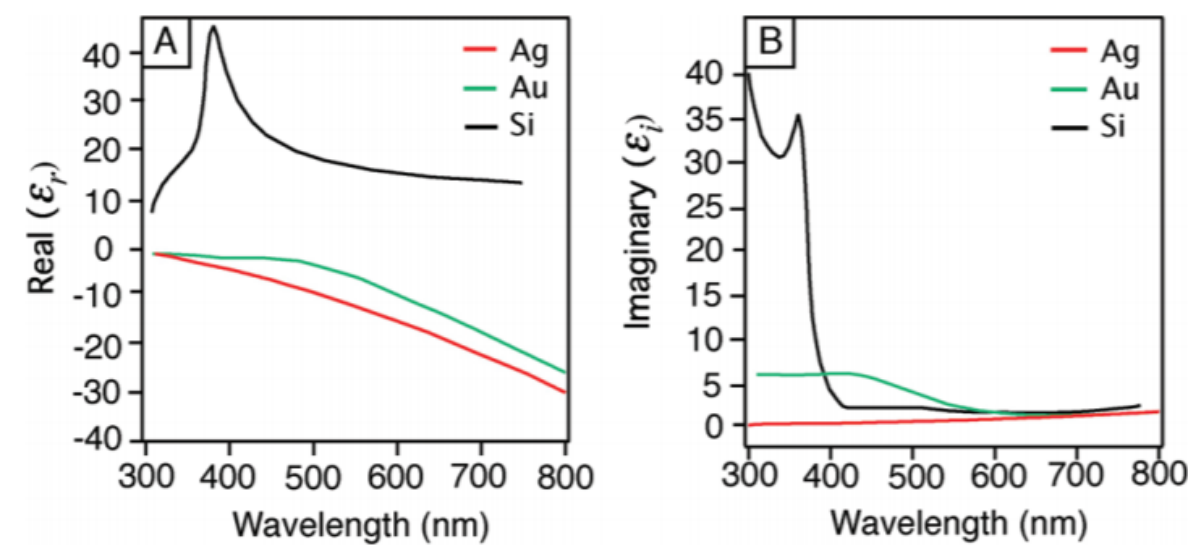

Figure 1.3: Plot of the (A) real, $\varepsilon_{r}$, and (B) imaginary, $\varepsilon_{i}$, components of the dielectric function of $A g, A u$, and Si as a function of wavelength. Reproduced with permission from (19). Copyright American Chemical Society 2011. 


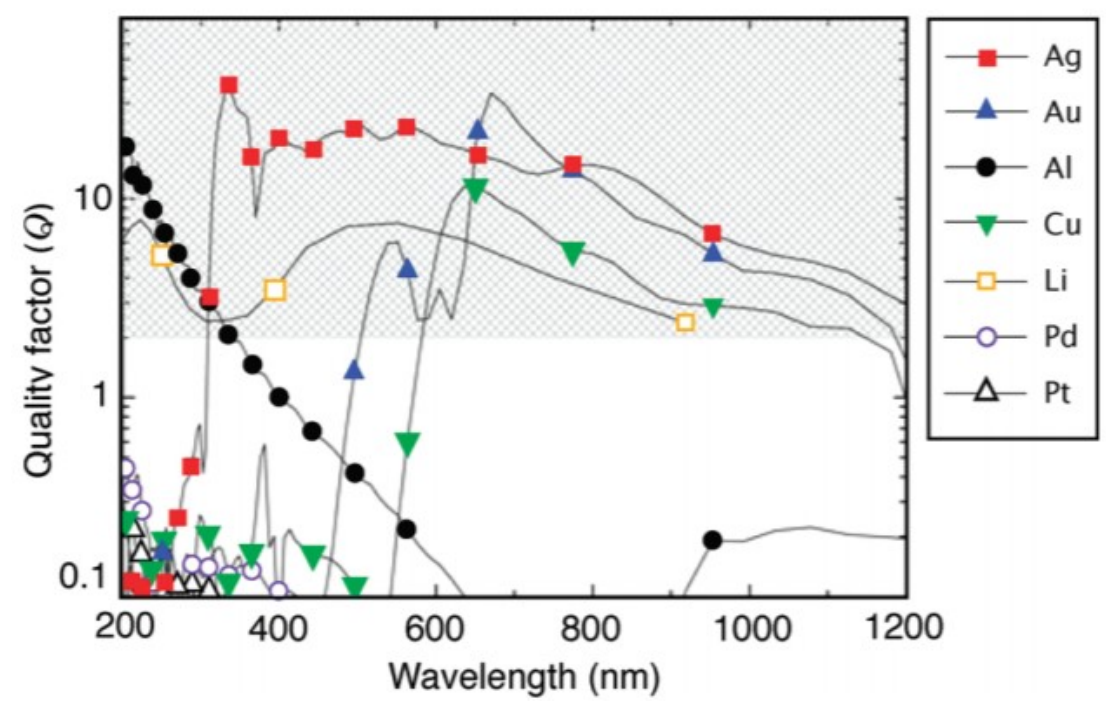

Figure 1.4: Quality factor (Q) of the LSPR for a metal/air interface. A higher $Q$ denotes less damping and a stronger plasmon resonance. The shaded area represents the region of interest for many plasmonic applications. Reproduced with permission from (19). Copyright American Chemical Society 2011.

Ag nanoparticles, depending on their size and shape, can support LSPR with a range a of different frequencies but are bioactive and highly prone to oxidation. Au nanoparticles on the other hand can only support LSPR with limited frequencies but are bioinert. Consequently, Ag nanoparticles are more popular for niche plasmonic applications and Au nanoparticles are a more popular choice for biological and biomedical applications of plasmonic nanoparticles. ${ }^{2,19}$ Because this study requires nanoparticles with well defined plasmons over a wide range of visible wavelengths, $\mathrm{Ag}$ is selected as the material of choice.

\subsubsection{Effect of Environment on LSPR}

Another variable that effects the LSPR of nanoparticles is the dielectric properties of their surrounding media; particularly the refractive index, $n$, of any matter close to the nanoparticle surface ${ }^{3,25-27,28}$

To evaluate the LSPR frequency's dependence on the surrounding medium's dielectric function a frequency dependent form of the real component of the metal's dielectric function can be used: 


$$
\varepsilon_{r}=1-\frac{\omega_{p}^{2}}{\omega^{2}+\gamma^{2}}
$$

Where $\omega_{p}$ is the plasmon frequency of the bulk metal described in Equation 1, $\omega$ is the frequency of incident radiation and $\gamma$ is the damping frequency of the bulk metal. ${ }^{3}$ In the UV-Vis region of the electromagnetic spectrum $\gamma<<\omega$, which allows the above equation to be simplified to ${ }^{3}$

$$
\varepsilon_{r}=1-\frac{\omega_{P}^{2}}{\omega^{2}}
$$

Rearranging Equation 20 and using the resonance condition $\left(\varepsilon_{1} \approx-2 \varepsilon_{m}\right)$ we obtain

$$
\omega_{\max }=\frac{\omega_{P}}{\sqrt{2 \varepsilon_{m}+1}}
$$

Where $\omega_{\max }$ is the peak LSPR frequency, $\omega_{P}$ is the bulk plasmon frequency and $\varepsilon_{m}$ is the dielectric constant of the medium. Converting from frequency to wavelength using $\lambda=\frac{2 \pi c}{\omega}$, and from dielectric constant to index of refraction using $\varepsilon_{m}=n_{m}{ }^{2}$, Equation 21 becomes

$$
\lambda_{\max }=\lambda_{P} \sqrt{2 n_{m}^{2}+1}
$$

where $\lambda_{\max }$ is the LSPR peak wavelength, $\lambda_{P}$ is the wavelength corresponding to the plasma frequency of the bulk metal and $n_{m}$ is the refractive index of the medium. ${ }^{3}$ This equation tells us that the plasmon peak position will increase (or "red-shift") as the refractive index of the environment increases. ${ }^{3}$

While the relationship between $\lambda_{\max }$ and $n_{m}$ is not strictly linear, it can be approximated as linear for the small range of $n_{m}$ encountered in practical experiments. Figure 1.5 shows experimental data indicating the effect that using solvents with different refractive indices has on the LSPR peak of $\mathrm{Ag}$ and $\mathrm{Au}$ nanocubes. As can be seen in Figure 1.5e, the experimentally measured shift in the plasmon peak is very close to the plasmon shift calculated using the equations outlined above derived from the Drude model. 

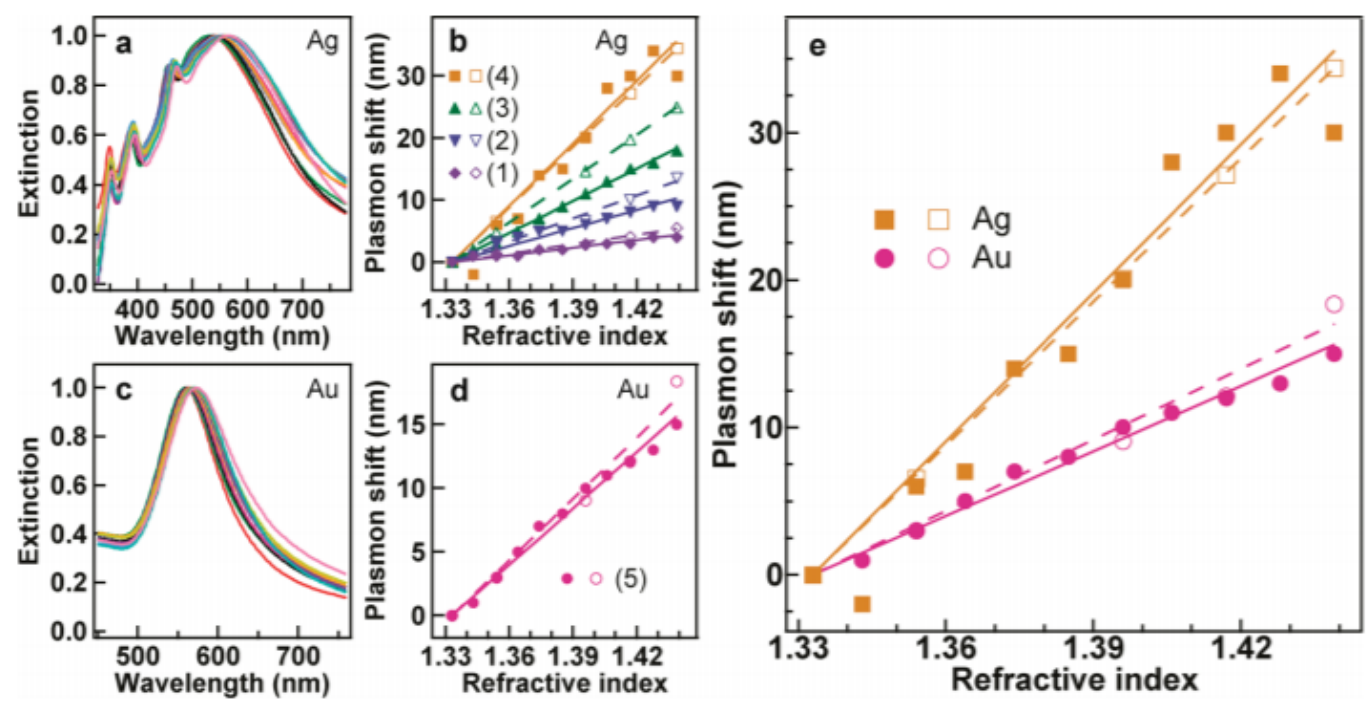

Figure 1.5: Extinction spectra of the Ag nanocubes acquired by dispersing the nanocubes in the different solvent mixtures. (b)

Shifts of the different plasmon resonance modes of the Ag nanocubes as a function of the refractive index. (c) Extinction spectra of Au nanocubes acquired by dispersing the nanocubes in the different solvent mixtures. (d) Shifts of the plasmon mode of the Au nanocubes as a function of the refractive index. The numbers in (b) and (d) indicate the different plasmon modes as in Figure $2 a$ and $b$. (e) Comparison of the refractive index sensitivity of the dipolar plasmon mode between the Ag and Au nanocubes. The solid and hollow symbols represent the measured and calculated plasmon shifts, respectively. The lines are linear fits. Reproduced with permission from (28). Copyright American Chemical Society 2011.

LSPR red-shifting in response to an increase $n_{m}$ occurs for all size and shape of the nanoparticle and also occur in the presence of adsorbed species on the nanoparticle surface. ${ }^{8,25,26}$ Larger particles or particles with a larger effective radius will exhibit a greater sensitivity to changes in $n_{m}$, making large particles more popular for sensing applications..$^{25}$

\subsubsection{Effect Size of Nanomaterial on LSPR}

The LSPR of a nanoparticle will broaden and red-shift as the size of the nanoparticle increases. This has been shown to be the result of radiation damping and dynamic depolarization. ${ }^{29-31}$ Radiation damping arises from spontaneous emission of radiation by the induced dipole on the nanoparticle, leading the electrons to oscillate at a lower energy following said emission. ${ }^{29}$ This emission increases as the particle 
size increases which reduces the size of the induced dipole and increases the spectral line-width of the plasmon. ${ }^{29}$ Dynamic depolarization, the phenomena responsible for red-shifting, is the result of the depolarization of the electron cloud across the surface of a particle caused by the increase in the ratio of particle size to wavelength. ${ }^{29,31,32}$

As the size of a nanoparticle changes, the types of plasmonic modes it can support also changes. Nanoparticles with diameters of $<30 \mathrm{~nm}$ will have a homogenously distributed surface charge after being excited with incident light, meaning that they have only one possible plasmon resonance, a dipolar mode. 30,33 Nanoparticles with diameters $>30 \mathrm{~nm}$ will experience non-homogenously distributed surface charge after being excited leading to higher order charge distributions. ${ }^{30,32,33}$ The higher order mode most commonly observed in the extinction spectra of larger particles is the quadrupolar mode, but even higher order modes can exist depending on a particle's shape (discussed in section 1.1.6). The peaks corresponding to higher order LSPR modes are always located at shorter wavelength values than the dipolar mode, and in the presence of higher order modes the dipolar mode is often red-shifted to a longer wavelength value due to the presence of higher order EM field distributions. ${ }^{30}$

The effects of radiation damping, dynamic depolarization and higher multipoles that come in to play as nanoparticle size increases can cause significant shifts in the LSPR peak of nanoparticles. For example, the LSPR peak of $20 \mathrm{~nm}$ silver nanoparticles in water is at $520 \mathrm{~nm}$, but, if the size of the silver nanoparticles is increased to $100 \mathrm{~nm}$, the LSPR red-shifts all the way to $600 \mathrm{~nm} .{ }^{32}$ Because the frequency of the LSPR of nanoparticles is so dependent on their size, researchers can specifically synthesize nanoparticles of specific sizes to achieve specific LSPRs tuned for niche applications.

\subsubsection{Effect of Shape on LSPR}

Along with chemical composition and size, the shape of a nanoparticle also plays an important role in dictating its optical properties. ${ }^{3,19,29,30,34-36}$ The geometry of a nanoparticle alters how charge is distributed 
at the surface of a particle and thus the type of plasmon modes (dipolar, multipolar, etc) that the particle can support. This results in different shapes of particles having distinct LSPR patterns and very different extinction spectra as can be seen in Figure 1.6.

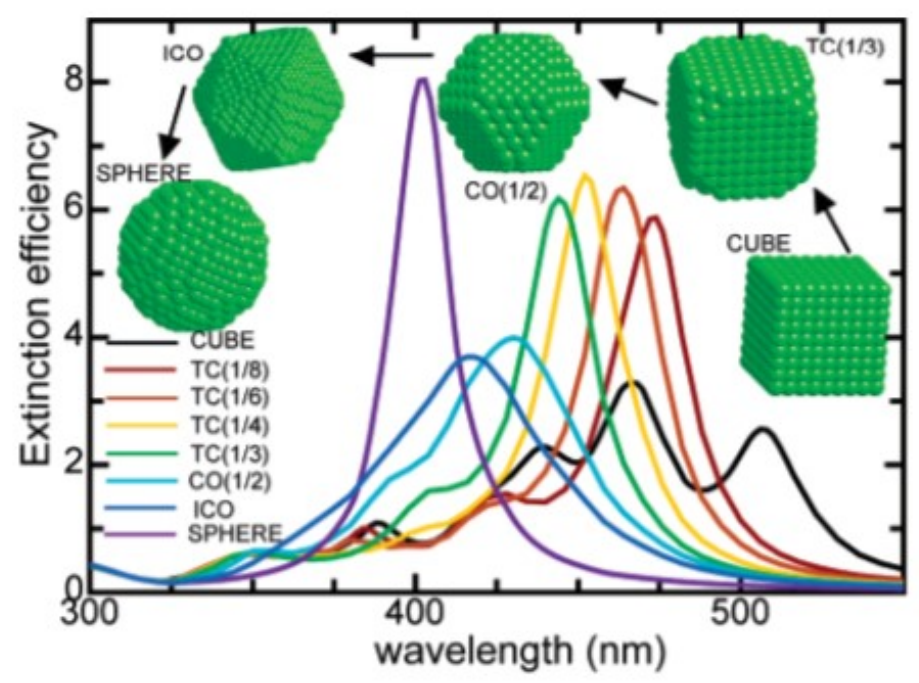

Figure 1.6: Extinction efficiencies as a function of the wavelength of the incident light of a silver cube, different truncated cubes, and a spherical nanoparticle. Reproduced with permission from ( $\left.{ }^{30}\right)$. Copyright American Chemical Society 2007.

Certain geometries of particles with lots of edges and corners, such as cubes, octahedrons and prisms, will have distinct extinction spectra distinguished by the presence of higher order modes at unique wavelength positions. ${ }^{33}$ Nanocubes have multiple peaks in their extinction spectra as charge accumulates at their edges and corners inducing extra multipolar charges. ${ }^{37}$ Silver nanocubes (AgNCs), such as the ones used in this study, typically exhibit 3 distinct peaks in their extinction spectra: a dipolar peak at 430-460 $\mathrm{nm}$, a quadrupolar peak at $\sim 390 \mathrm{~nm}$ and a multipolar peak at $\sim 350 \mathrm{~nm}$. The multipolar peak in the extinction spectra of silver nanocubes corresponds to a octupolar resonance which is unique to silver cubes. $^{28,38}$ The width and position of the higher order mode peaks in geometric nanoparticles are indicative of the sharpness of the corners and edges of the structure. The more truncated the corners or edges of the cube, the broader and more blue shifted the peaks will be. ${ }^{30}$ 


\subsubsection{Plasmonic Coupling}

When plasmonic nanoparticles are in close proximity to each other, such as in a concentrated solution or monolayer, the near-field of one nanoparticle can interact with that on the other particle. ${ }^{29,39-43}$ As discussed in section 1.1.1, the presence of an LSP causes an EM near-field on the surface of a particle that is enhanced compared to the incident light field. In a situation where two nanoparticles are very close (within $\mathrm{nm}$ of each other), the electric fields surrounding the respective particles can interact and the electric field $E$ felt by each particle is the sum of the incident light field $\left(E_{0}\right)$ and the near-field $\left(E_{n f}\right)$ of the neighboring particle. ${ }^{40}$

$$
E=E_{0}+E_{n f}
$$

This near-field interaction results in the plasmon oscillations of the two nanoparticles becoming coupled and leads to what are referred to as hybridized plasmons. ${ }^{39-42,44}$ According to the plasmon hybridization theory developed by Nortlander et al, when two plasmons modes interact they will mix to form two new, hybridized modes: an asymmetrically coupled anti-bonding mode and symmetrically coupled bonding mode. ${ }^{44}$ These hybridized plasmons can be depicted using a plasmon hybridization diagram that is very similar to the molecular orbital diagrams used to explain chemical bonding in molecules. ${ }^{45}$ Depending on the orientation of the incident EM radiation used the excite the LSPR of the particles, the creation of transverse or longitudinal coupling modes relative to the dimer axis are possible. For nanoparticles the interaction between transverse modes is significantly weaker than the longitudinal modes, and thus results in less significant field enhancement. Figure 1.7 below shows the plasmon hybridization diagram for the longitudinal dipolar plasmonic modes in spherical metal nanoparticles. 


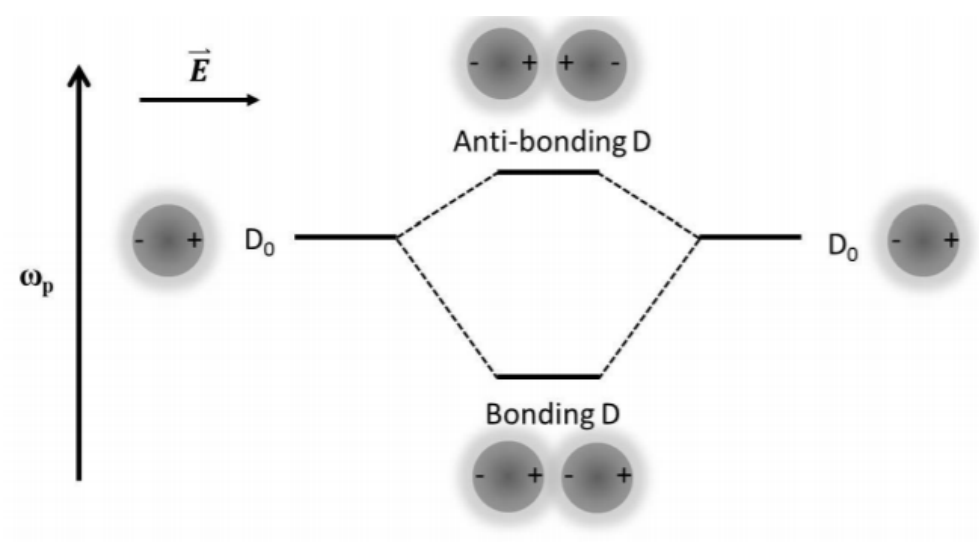

Figure 1.7: Plasmon hybridization scheme of longitudinal dipolar modes of an interacting nanoparticle dimer. Two interacting modes will mix to form an asymmetrically coupled anti-bonding mode and symmetrically coupled bonding mode. Reproduced with permission from (33). Copyright Daniel Prezgot 2014.

In the case of the longitudinal dipole modes shown in Figure 1.7, the anti-bonding mode has no net dipole moment and will not resonate with incident radiation. The bonding mode, however, will produce a strong resonance mode that is lower energy than the initial resonant mode of each individual particle. This new lower energy hybrid plasmon mode causes a red-shift in the LSPR peak. ${ }^{29,39-44}$

The magnitude of the plasmon wavelength shift has been shown by various researchers to be directly dependent on the interparticle distance. ${ }^{39,40,43-47}$ This is because a nanoparticle's near-field will decay exponentially with distance. ${ }^{3,40}$ As the distance between particles increases, the value of $E_{n f}$ in Equation 23 decreases, decreasing the total value of $E$ and decreasing the degree to which coupling occurs. This in turn results in a less red-shifted LSPR peak. ${ }^{39,40,43-47}$

The magnitude of the coupling induced plasmon-shift has also been shown to be affected by the size, shape and orientation of the nanoparticles. ${ }^{39,40,43,46,47}$ For dimers of spherical nanoparticles that are less than $80 \mathrm{~nm}$ in diameter, only the dipolar modes will undergo plasmon coupling; however, for larger nanospheres and more complexly shaped nanoparticles that support higher order plasmon modes, coupling between higher order modes (dipole-multipole, quadrupole-quadrupole etc) will occur. ${ }^{39,40,46,47}$ 
For geometric nanoparticles such as nanocubes, the orientation of the particle dimers also influences it's near field coupling behaviour. Figure 1.8 illustrates the calculated extinction spectra for two AgNC dimers with different orientations.
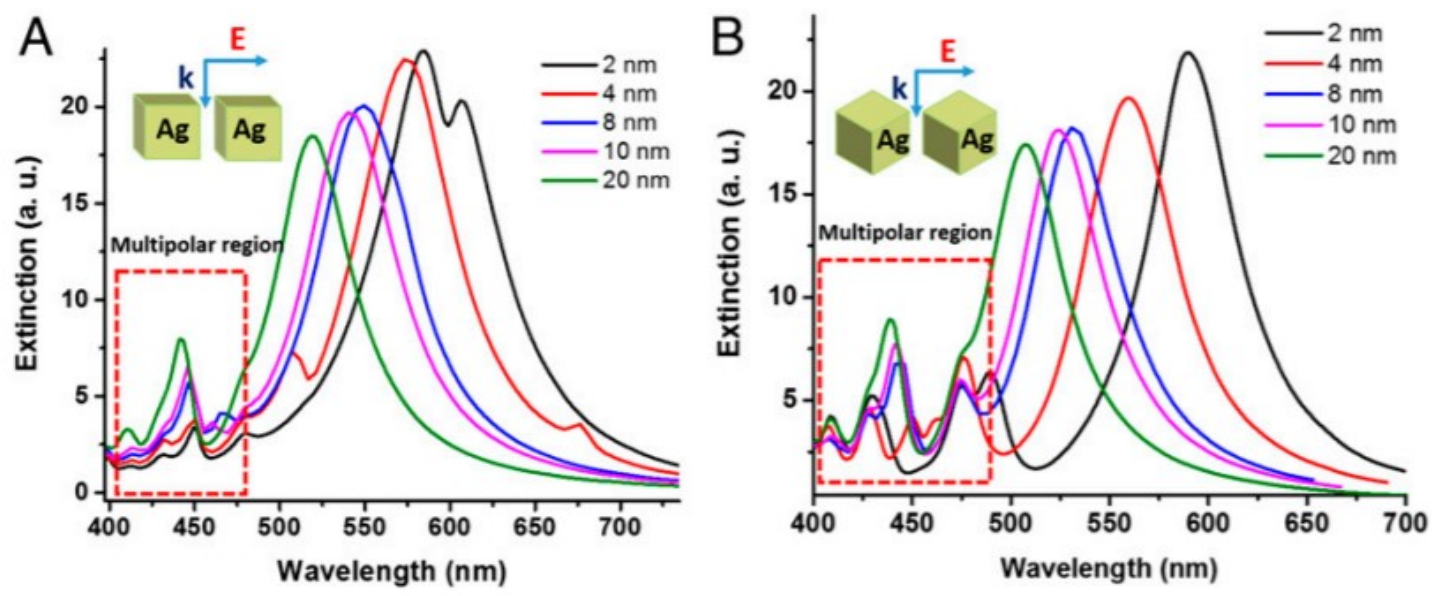

Figure 1.8: DDA calculated extinction spectra for the AgNCs in (A) Face-Face and (B) Edge-Edge orientations at varying separation distances of the dimer $(2,4,8,10$, and $20 \mathrm{~nm})$. The incident light is polarized along the interparticle axis. Reproduced with permission from (39). Copyright National Academy of Sciences 2019.

For the $2 \mathrm{~nm}$ separation spectra in Figure 1.8A the main peak in the spectrum (representing the coupling of dipolar resonances) is split. This is because when NCs are oriented face-to-face in a dimer there are two different dipoles coupling: the dipoles that correspond to the adjacent faces of the respective cubes and the dipoles that correspond to the adjacent corners of the respective cubes. ${ }^{39}$ The magnitude and direction of this splitting depends on the size and roundness of the cubes in the dimer and will typically only occur for interparticle distances of $\leq 2 \mathrm{~nm} .{ }^{48}$

Plasmonic coupling is often examined via simulations in the context of individual dimers or small clusters but most experimental nanoparticle arrays are often composed of billions of random nanocubes that exhibit various degrees of coupling. In nanocube monolayers, such as the ones used in this investigation, the interparticle distance fluctuates. Monolayers that are higher density will have more AgNC 
dimers/clusters while lower density monolayers may have predominately spatially resolved cubes that will not undergo any coupling.$^{49}$ The extinction spectrum of a low density monolayer will be essentially the same as that of an individual cube as the interparticle distance is too significant for plasmonic coupling to occur. For higher density monolayers there can be a range of interparticle distances so that some particles will undergo coupling and others will not. Because of the sheer number of nanoparticles in a monolayer, when some fraction of their population is well coupled the coupling can manifest as an additional peak in the extinction spectrum in the 580-600 $\mathrm{nm}$ region instead of a red-shift of the LSPR dipole peak.

\subsubsection{Substrate Induced Hybridization}

Significant shift of the LSPR of plasmonic nanoparticles can also occur when nanoparticles are supported by solid substrates. $3,8,26,29,38,50,51$ For supported nanoparticle assemblies two types of substrates are typically used: metallic and dielectric..$^{3,8,26,38,50}$ With metallic substrates the interaction between the LSPR of the nanoparticle and the substrate surface plasmons can result in hybridized plasmons that have both localized and propagating character..$^{50,52}$ Unlike metal surfaces, dielectric surfaces have no substrate modes; however, the nanoparticle still interacts with the substrate resulting in hybridized nanoparticle plasmon modes..$^{38,50,51}$

When plasmonic nanocubes are suspended in solutions they exhibit primarily one dipolar mode; when the same nanocubes are supported by a dielectric substrate, the existing dipolar mode becomes redshifted and a new peak in the extinction spectra emerges at a higher energy. ${ }^{38,50,51}$ This occurs because the presence of the substrate breaks the symmetry of the nanoparticles environment, inducing the coupling of the nanocubes plasmons modes to form hybridized bonding and antibonding modes.

For spherical nanoparticles in a vacuum there is no coupling between their dipolar and quadrupolar modes. ${ }^{38}$ However, when the nanoparticles are brought close to a dielectric substrate, their dipolar 
plasmon density induces surface image charges in the substrate. ${ }^{38,50,51}$ This image of a dipolar plasmon will have a significant quadrupolar field component which now allows for the coupling of the dipolar and quadrupolar plasmons..$^{38,50,51}$ This phenomena is known as substrate induced hybridization.

For geometric nanoparticles such as nanocubes, the substrate induced hybridization occurs to a much greater extent. This is due to two factors: the increased size of the image charge induced on the substrate and the increased number of plasmon modes present in a nanocube. ${ }^{38}$ For silver nanocubes, such as the ones used in this study, it has been shown that substrate induced hybridization leads to red-shifting of the existing dipolar mode and the emergence of a new plasmon mode on the blue side of the dipolar mode. ${ }^{51}$ Nanocubes have two intrinsic plasmon modes: a dipolar mode $\left(D_{0}\right)$ which is a bright mode and a quadrupolar mode $\left(\mathrm{Q}_{0}\right)$ which is a dark mode. A plasmon mode is referred to as a bright plasmon mode if it possesses finite dipole moments and thus can be excited by visible light. ${ }^{38}$ Because bright plasmon modes can be excited by visible light they can be observed and analyzed using UV-Vis spectroscopy. Dark plasmon modes possess no dipole moments, and thus cannot be excited by visible light, or observed individually by UV-Vis spectroscopy. ${ }^{38,40}$ Because the $D_{0}$ and $Q_{0}$ modes of nanocubes are nearly degenerate they interact very easily forming new hybridized modes. ${ }^{38}$ Figure 1.9 below illustrates a plasmon coupling diagram similar to the one shown in section 1.1.8 that illustrates the substrate-induced hybridization of the $\mathrm{D}_{0}$ and $\mathrm{Q}_{0}$ modes of a AgNC. ${ }^{38}$ 
a
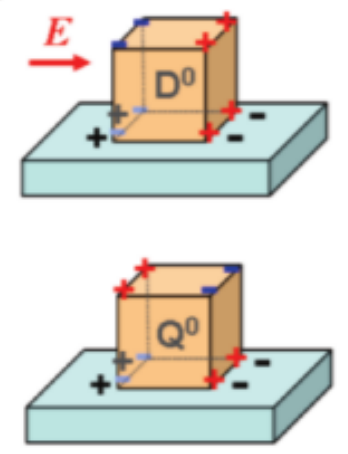

b

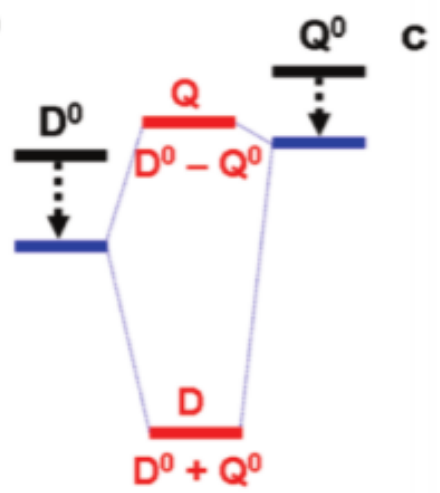

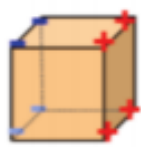

$D^{0}$

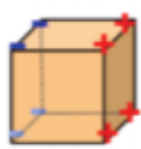

$\mathbf{D}^{0}$
$+$

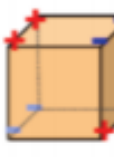

$\mathbf{Q}^{0}$

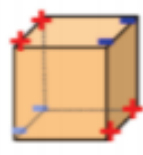

$\mathbf{Q}^{0}$

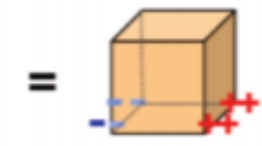

$D^{0}+Q^{0}$

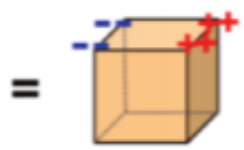

$D^{0}-Q^{0}$

Figure 1.9: Schematic illustration of (a) intrinsic cube modes with induced hybridization from the dielectric; (b) energy diagram of dielectric effect where the black dotted lines represent the pure dielectric screening effect and the thin blue lines represent the substrate mediated interaction; (c) charge distribution of fully hybridized modes. Reproduced with permission from (38). Copyright American Chemical Society 2011.

As can be seen in Figure 1.9, the mixing of the $D_{0}$ and $Q_{0}$ modes results in the formation of a bonding $\left(D_{0}+Q_{0}\right)$ and anti-bonding $\left(D_{0}-Q_{0}\right)$ mode. Because the bonding mode $\left(D_{0}+Q_{0}\right)$ originates mainly from the $D_{0}$ mode, it is referred to as the hybridized bonding dipolar (D) mode. Similarly, the anti-bonding mode $\left(D_{0^{-}} Q_{0}\right)$ is referred to as the hybridized anti-bonding quadrupolar $(Q)$ mode as it originates mainly from the $Q_{0}$ mode.

The position of the $\mathrm{D}$ and $\mathrm{Q}$ hybridized modes in the extinction spectra are dependent on the distance of the AgNC from the substrate surface. ${ }^{38,51}$ Figure $1.10 \mathrm{~A}$ shows FDTD simulations evaluating the scattering patterns of a single $90 \mathrm{~nm} \mathrm{AgNC}$ as it approaches a glass substrate. These spectra show that as the particle approaches the surface the dipole mode associated with the solution spectrum red-shifts and a blue peak indicating the presence of a quadrupolar mode appears at $430 \mathrm{~nm} .^{51}$ 

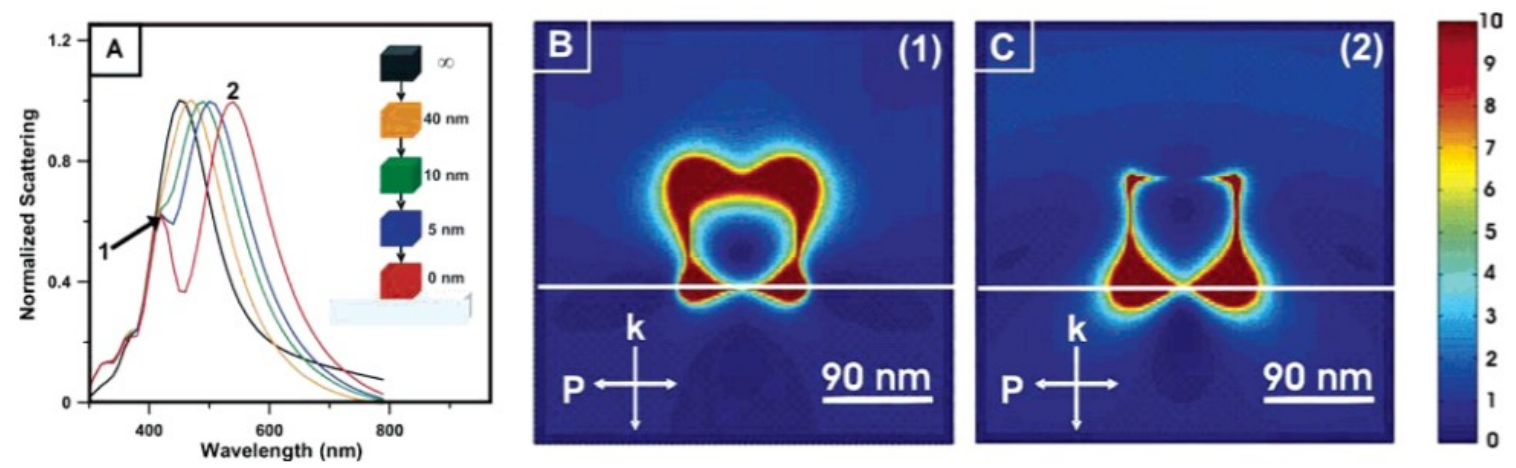

Figure 1.10: FDTD theory showing (A) the emergence of a second peak as a single nanocube (90-nm diameter) approaches a dielectric substrate, and $(B, C)$ the field intensities for peaks 1 and 2 of the nanocube in contact with the substrate (the white line in the field pattern images represent the substrate). Reproduced with permission from ${ }^{(51)}$. Copyright American Chemical Society 2005.

Another important consequence of the substrate-hybridized modes is that the modes display different locations of their charge density. ${ }^{38,50,51}$ Figure $1.10 \mathrm{~B}$ and $\mathrm{C}$ represent the calculated electromagnetic field enhancements of the $Q$ and $D$ modes respectively. The hybridized $D$ mode is oriented towards the substrate while the hybridized $\mathrm{Q}$ mode it oriented primarily away from the substrate. This can be leveraged for many sensing applications as the D more is particularly sensitive to the refractive index of the substrate while the $Q$ mode is particularly sensitive the refractive index of the surrounding environment. $^{35,38}$

\subsection{Thermoplasmonics}

Early plasmonic experiments documented a local increase in temperature around particles that had been optically excited. ${ }^{53-55}$ This localized heating was initially dismissed as a side effect of LSPR excitation that should be minimized, however, scientists later realized that this light-controlled localized heating provided an unprecedented way of controlling thermal energy at the nanoscale. ${ }^{53,54}$ This realization led to the birth of thermoplasmonics, the branch of plasmonic science that investigates the heat produced by plasmonic nanomaterials and any potential applications of this effect. ${ }^{53,54}$ Despite the fact that 
thermoplasmonics has been around for twenty years, nanoscale photothermal processes are still not fully understood..$^{54,56}$ Many theoretical simulation techniques have been developed in hopes of mathematically explaining nanoscale localized heating, and while several have been shown to be reasonably accurate, no single explanation is accepted within the scientific community as conclusively explaining the complex underlying physics of the phenomena. ${ }^{54,57}$ Though the complex relationship between plasmonic excitation and heat generation is not yet fully understood, the general mechanism of photothermal heating is known. This mechanism, along with the variables that affect it, are explained below.

\subsubsection{Mechanism of Photothermal Heat Generation in Nanoparticles}

When plasmon electrons are excited to oscillation they will undergo electron-electron and electronphonon collisions within the metal lattice. ${ }^{55,58,59}$ These collisions produce heat energy which then spreads throughout the nanoparticle. ${ }^{56,59}$ This phenomenon is shown in Figure 1.11 below.
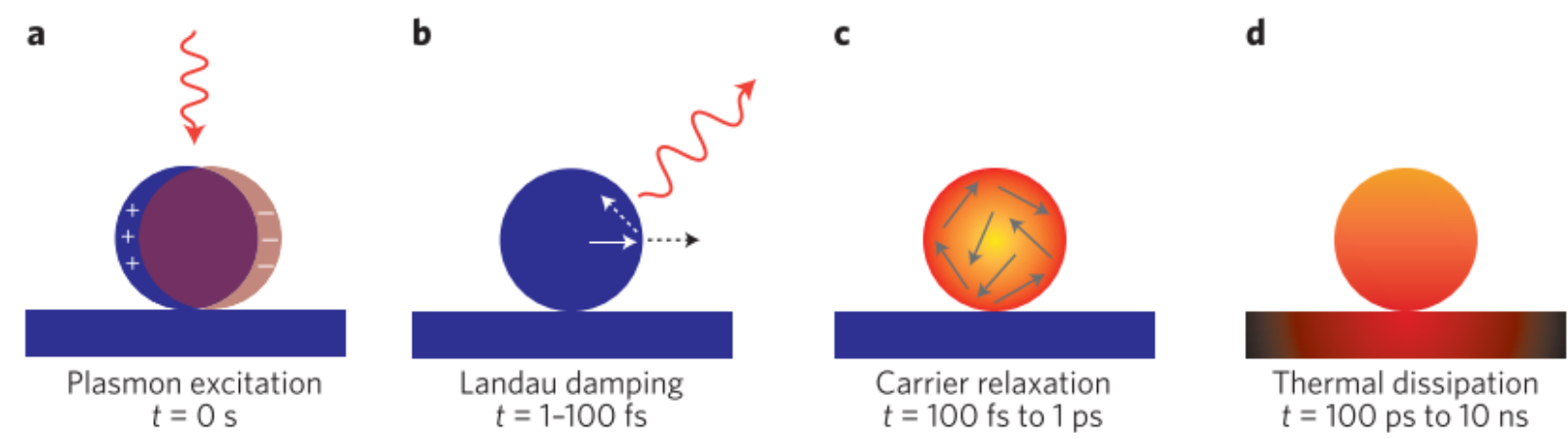

Figure 1.11: Schematic representation of the lifetime of a plasmon resonance. Upon the plasmon's excitation (a) the excited electrons will form a thermal population of hot carriers, some of which can escape the nanoparticle as hot-electrons (b) others of which will undergo collisions within the metal lattice (c). These collisions will produce heat which will thermally dissipate through the nanoparticle (d). Adapted with permission from (60). Copyright Nature 2015.

Once the LSPR of a plasmonic nanoparticle is excited, it only takes a picosecond for the whole nanoparticle to become heated. ${ }^{56}$ Because metals have very high thermal conductivity, this heat is rapidly dissipated 
to the local surrounding environment until an equilibrium temperature is reached. ${ }^{61}$ The rapid photoinduced heating followed by thermalization is referred to as the thermoplasmonic effect and is a photothermal process.

Photothermal heating can occur in conjunction with other photoinduced processes, such as the hotelectron generation shown in Figure 1.12b. ${ }^{56,58,60,62-64}$ Similar to the complex process of plasmonic heat generation, the process of hot-electron generation in plasmonic nanomaterials is not fully understood. ${ }^{63-}$ ${ }^{68}$ Though hot-electron generation is not a focus of this study, it is worth noting that many reactions that had initially been attributed to hot-electron generation, are now being hypothesized to occur as a result of photothermal effect instead. ${ }^{69-71}$ While this puts a damper on the potential of hot-electron science, it strengthens the prospective of thermoplasmonics.

\subsubsection{Temperature Increase of Thermoplasmonic Particles}

The photothermal temperature increase around a plasmonic nanoparticle can be described by the following equation developed by Baffou et al

$$
\Delta T=\frac{P_{a b s}}{4 \pi \beta R_{e q} k}
$$

where $P_{a b s}$ is the power of the radiation absorbed by the nanoparticle, $\beta$ is a shape correction coefficient, $R_{e q}$ is the radius of the nanosphere with equivalent volume to the desired particle size and $k$ is the thermal conductivity of the medium. ${ }^{72}$ Though this equation was initially developed for spherical nanoparticles, it serves as a reasonable approximation for other shaped nanoparticles, such as the AgNC used in this study. ${ }^{72}$ Because the power of the radiation absorbed by the nanoparticle is equivalent to the intensity of the incident radiation multiplied by the absorption cross section of the particle, the equation can be rewritten as

$$
\Delta T=\frac{\sigma_{a b s} I}{4 \pi \beta R_{e q} k}
$$


where $\sigma_{a b s}$ is the absorption cross section, and $I$ is the intensity of the incident radiation used for plasmon excitation. ${ }^{72}$ Plasmonic nanoparticles have exceptionally high absorption cross sections: in fact the absorption cross section of a plasmonic nanoparticle can be more than four times the geometric radius of the particle. ${ }^{73}$ It is these extremely large cross sections that makes plasmonic nanoparticles so ideal for thermal heating: the greater the value of $\sigma_{a b s}$ the greater the increase in localized temperature. ${ }^{61}$

The shortcoming of Equations 24 and 25 is that they only describe the localized heating around a single nanoparticle. While this works well for a single nanoparticle system, it does not accurately describe what occurs for nanoparticle assemblies where more than one nanoparticle is optically excited simultaneously. ${ }^{54,74}$ In 2D arrays of nanoparticles there are two distinct photothermal regimes: a temperature confinement regime where temperature increase is observed only in the vicinity of individual particles and a temperature delocalization regime where the temperature increase is smooth across the whole surface of the array. ${ }^{54,74}$ Temperature delocalization occurs as a result of collective photothermal effects and leads to strong temperature increase many units larger than that predicted by Equation $25 .{ }^{54,74}$ Baffou et al invented a dimensionless number, $\xi$, that predicts the extent to which collective photothermal will occur. ${ }^{54,74}$ According to Baffou et al

$$
\xi=p^{2} / 3 R D
$$

where $p$ is the mean first-neighbour interparticle distance, $R$ is the radius of the nanoparticles, and $D$ is the characteristic size of the nanoparticle array under illumination. Collective effects become dominant when $\xi<1$ which can be achieved by decreasing the interparticle distance, increasing nanoparticle size, or increasing the size of the array under illumination.

\subsubsection{Effect of Composition, Size, and Shape on Thermoplasmonic Properties}


There are several variables that need to be considered when it comes to selecting the most appropriate nanoparticle for thermoplasmonic applications. ${ }^{55}$ Depending on nature of the application of the thermoplasmonic material, different needs (thermal stability, biocompatibility, etc) need to be considered. $^{54,55}$ Relevant to all applications of thermoplasmonics is the importance of selecting nanoparticles that maximize light-to-heat conversion. ${ }^{54}$ The easiest way to do this is to increase nanoparticle size, as for small nanoparticles $(<60 \mathrm{~nm}) \sigma_{a b s} \propto$ volume. ${ }^{54}$ Even for larger particles (>60 nm) increasing size leads to increase in temperature at constant wavelength, although beyond a certain size threshold not all of the volume of the particle contributes to the photothermal process. ${ }^{55,75,76}$

To isolate the effect of shape and composition, the $\sigma_{a b s}$ of small nanoparticles are normalized by their volume $(V)$ to create the Joule parameter, Jo which is defined as ${ }^{76}$

$$
J o=\frac{\sigma_{a b s} \lambda_{r e f}}{2 \pi V}
$$

where $\lambda_{\text {ref }}$ is set arbitrarily to $\approx 1240 \mathrm{~nm}$ so that $J o$ is dimensionless. While other methods divide the $\sigma_{a b s}$ by the projected surface of the nanoparticle to obtain a dimensionless number that can be used for quantifying absorption efficiency, using $J o$ has the advantage that it works for any nanoparticle geometry not solely spheres. ${ }^{76}$ The $J o$ value calculated for various types of nanoparticle at different wavelengths can be seen in Figures 1.12 and 1.13 below. 

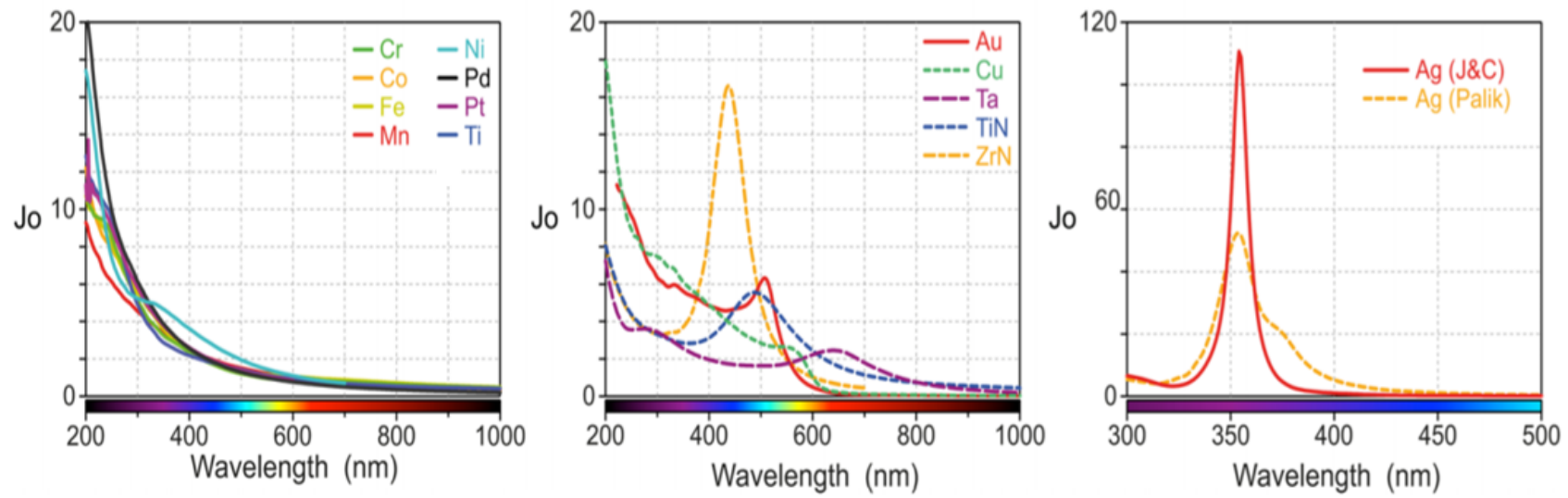

Figure 1.12: Spectra of the Joule numbers for a large set of materials. Materials have been arranged in families: Plasmonic resonance out of the visible range (left), in the visible range (middle), and the singular case of silver (right) where data have been plotted considering two distinct data sets (the optical constants of Johnson and Christy, and of Palik's handbook).

Adapted with permission from (76). Copyright American Chemical Society 2015

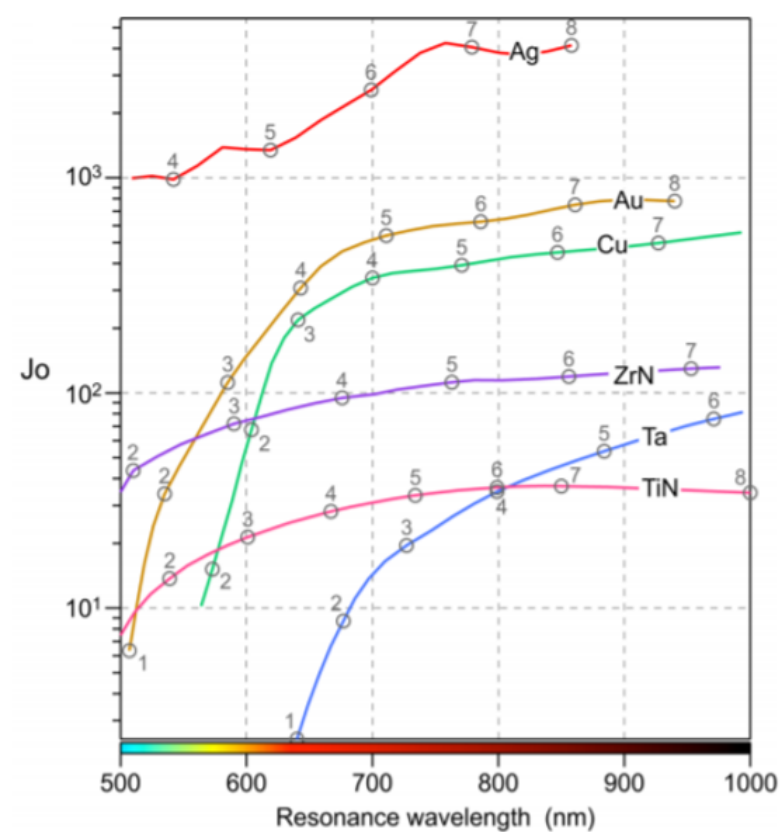

Figure 1.13: Calculated Jo number plotted as a function of the resonance wavelength of ellipsoids made of various materials.

Adapted with permission from (76). Copyright American Chemical Society 2015 
As can be seen in Figures 1.12 and 1.13, the photothermal efficiency for Ag is orders of magnitude higher than other materials in the visible range. This high photothermal efficiency is what makes Ag one of the preferred plasmonic materials for photothermal experiments and an appropriate choice for this investigation.

Figure 1.13 also illustrates how the shape of a nanoparticle also effect its thermoplasmonic properties. The more elongated a particle is, the more redshifted its LSPR wavelength is, and thus the longer wavelength of light required for thermoplasmonic heating. ${ }^{53,76,77}$ Because plasmon coupling leads to a redshift in the resonance wavelength, nanoparticle dimers and clusters that undergo plasmon coupling also require longer wavelengths of light to be thermoplasmonically excited..$^{78,79}$

\subsubsection{Applications of Thermoplasmonics}

Since they were first used by Huttman and Birngruber in 1999 as a heat source to denature proteins plasmonic nanostructures as nanosources of heat have shown many promising applications in photothermal therapy, drug delivery, sterilization, imaging and microscopy, efficient radiation heating, printing and nanoscale patterning, light-harvesting, and biosensing. ${ }^{57,61,86,72,74,80-85}$ Nanomaterials are advantageous as sources of heat because their heating can be controlled remotely with high efficiency and spatial resolution and they can be easily designed for specific applications. Photothermal therapy that uses plasmonic nanoparticles to target cancer tissues is far less invasive than surgical resection, it does not produce any harmful photochemical reaction intermediates, and it requires very short laser pulse times. ${ }^{81,85,87,88}$ Photothermal imaging and spectroscopy exploit the nanoscale size and femtosecond heating of plasmonic nanoparticles to achieve very high spatial and temporal resolution. ${ }^{75,82,89}$ Arrays and solutions of plasmonic nanostructures increase the efficiency of energy absorption for larger systems. ${ }^{90}$ Nanoscale printing can be accomplished by photothermally exciting plasmonic nanomaterials on polymer films with a controlled laser to embed a pattern. ${ }^{80,84}$ This study focuses on developing a method to achieve 
a selective thermoplasmonic embedment pattern, the particulars of which will be explained in more detail in section 1.4.2.

\subsection{Synthesis of Silver Nanocubes}

A variety of methods and techniques can be used to synthesize silver nanoparticles including laser ablation, gamma irradiation, electron irradiation, chemical reduction, photochemical methods, microwave processing, and biological synthetic methods. ${ }^{5,19,91,92}$ Some of these techniques, such as laser ablation, use top-down synthesis (making nanoparticles from breaking down bulk material), but typically bottom-up (making nanoparticles from individual atoms) solution synthesis are the most common. ${ }^{91-93}$ The first silver nanoparticles to be synthesized in solution were nanospheres, but over the past decade shape controlled synthesis techniques have developed significantly so that silver nanocrystals in a wide variety of geometric shapes can be easily made..$^{5,19,94,95}$ Though solution based synthetic methods differ in the chemical reagents, procedures and technique used and the morphology of nanoparticles produced, they all share a common approach. Silver nanoparticle synthesis techniques typically involve a silver salt precursor $\left(\mathrm{AgNO}_{3}\right.$ is the most common but others such as $\mathrm{CF}_{3} \mathrm{COOAg}$ are also used), a reducing agent, and a capping agent that helps stabilize the nanoparticles and may help direct their shape. ${ }^{19,91,92,96}$ Three common methods used to synthesize silver nanoparticle are the citrate reduction method, the sodium borohydride reduction method and the polyol method. ${ }^{19,91,94-97}$

The citrate reduction method is one of the oldest techniques used to synthesize silver nanoparticles and it is still popular today. ${ }^{98-101}$ In this method sodium citrate is added to a boiling solution of $\mathrm{AgNO}_{3}$ and it acts as the reducing agent and the stabilizing agent. ${ }^{97}$ This method is popular because it is easy to perform and the reaction is simple. However, silver colloids produced by the citrate reduction method tend to have polydispersed size and shape, and thus are unsuited for complex plasmonic applications where specific control of shape and/or size is important. ${ }^{97}$ 
The sodium borohydride method involves adding a cold solution of $\mathrm{NaBH}_{4}$ to aqueous silver precursor to produce small spherical silver nanoparticles. ${ }^{102-108}$ This method is widely used because it is cheap, it only requires two reagents (a silver precursor and the $\mathrm{NaBH}_{4}$ ), it produces nanoparticles very quickly and the progression of the reaction can be easily monitored by change in colour of the solution. ${ }^{102-108}$ The method works well for experiments that require concentrated solutions of small silver nanoparticles but just like the citrate reduction method the size and the shape of the particles are not consistently uniform. ${ }^{109}$

The polyol method involves heating a polyol (an organic compounds with many hydroxyl groups) with a salt precursor and a polymeric capping agent. ${ }^{94,95}$ In this method the polyol acts as both the solvent and the principle reducing agent. It is highly popular due to its versatility as it can be used to generate metal nanostructures of a variety of shapes and sizes. ${ }^{19,110}$ By varying reaction conditions such as reagent concentration, trace ion concentration, precursor injection rate, capping agents and solution temperature, the nucleation and growth processes can be controlled, and thus so can the shape and size of the resulting nanoparticles. ${ }^{19,91,110,111}$ The polyol method can be used to synthesize silver bipryamids, nanobars, polyhedrons, spheroids, triangular plates and nanowires, and it is the method of choice used to synthesize the AgNCs employed in these investigations. ${ }^{19,112}$ In this specific polyol method ethylene glycol (EG) is the polyol, $\mathrm{AgNO}_{3}$ is the silver precursor, polyvinyl pyrrolidinone (PVP) is the capping agent, $\mathrm{NaCl}$ is an etchant and $\mathrm{Na}_{2} \mathrm{~S}$ is a catalyst. The various steps in the reaction and their supporting theory are explained in detail in the following sections.

\subsubsection{Heating of Ethylene Glycol and Reduction of Silver}

The first step in the polyol synthesis of silver nanocubes is heating ethylene glycol to $160{ }^{\circ} \mathrm{C} .19,94,110,111$ Whenever $\mathrm{EG}$ is heated above $120^{\circ} \mathrm{C}$ in the presence of $\mathrm{O}_{2}$ it will lead to the formation of glycoaldehyde (GA) via the oxidation reaction seen below ${ }^{19,111,113}$ 


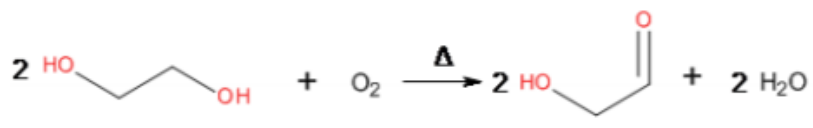

Reaction 1

Once glycoaldehyde is formed, it acts as the reducing agent for silver via reaction 2

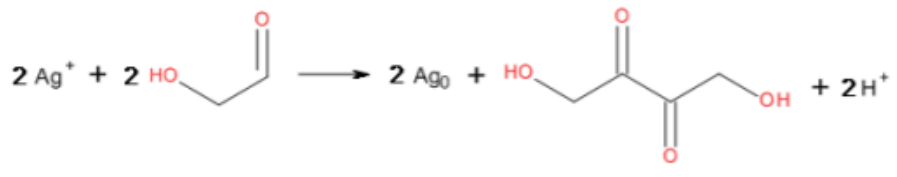

Reaction 2

The amount of metal precursor reduced depends directly on the concentration of GA. ${ }^{113}$ Because the amount of GA formed depends on the temperature of the reaction, the heating and temperature control of the EG solution play a big role in the success of the synthesis. ${ }^{19,113}$ Below $120{ }^{\circ} \mathrm{C}$ no GA will be formed, and while EG can itself act as a reducing agent for silver, leading to the production of some silver nanoparticles, this process is not nearly as efficient. ${ }^{113}$ Conversely, when the temperature of the reaction is too high, the reduction of silver can no longer be controlled to the same extent and nanoparticles with irregular shapes are produced. ${ }^{111,113}$ Spectroscopic studies of the GA reduction mechanism and evidence from experimental synthesis indicate that $160^{\circ} \mathrm{C}$ is the optimal temperature for maximizing the reduction of silver nitrate by GA and the yield of AgNCs. ${ }^{19,111,113}$

\subsubsection{Nucleation of Nanoparticles}

As the reduction of silver nitrate by GA progresses, the solution quickly become saturated with $\mathrm{Ag}^{0}$. However, even at the saturated concentration $\left(C_{S}\right)$ of $\mathrm{Ag}^{0}$ the formation of solid seed nuclei is too energetically costly. ${ }^{114,115}$ According to the LaMer model of colloidal nanocrystal formation, solid seed nuclei will only form once a minimum critical concentration $\left(C_{\min }\right)$ of $\mathrm{Ag}^{0}$ is reached, at which point the energy change associated with decreased entropy becomes accessible by the system. ${ }^{114,115}$ Once the $\left[\mathrm{Ag}^{0}\right]$ $=C_{\min }$ stable nuclei are formed, and provided $\left[\mathrm{Ag}^{0}\right]>C_{S}$, nucleation and growth continue simultaneously. If $\left[\mathrm{Ag}^{0}\right]$ drops below $C_{s}$, nucleation ceases and growth continues; however, usually this is not an issue as 
there is sufficient $\mathrm{GA}$ and $\mathrm{Ag}^{+}$ions in the solution to sustain a continued supply of $\mathrm{Ag}^{0}$. Figure 1.14 below summarizes the LaMer model outlining the progression of nanocrystal growth.

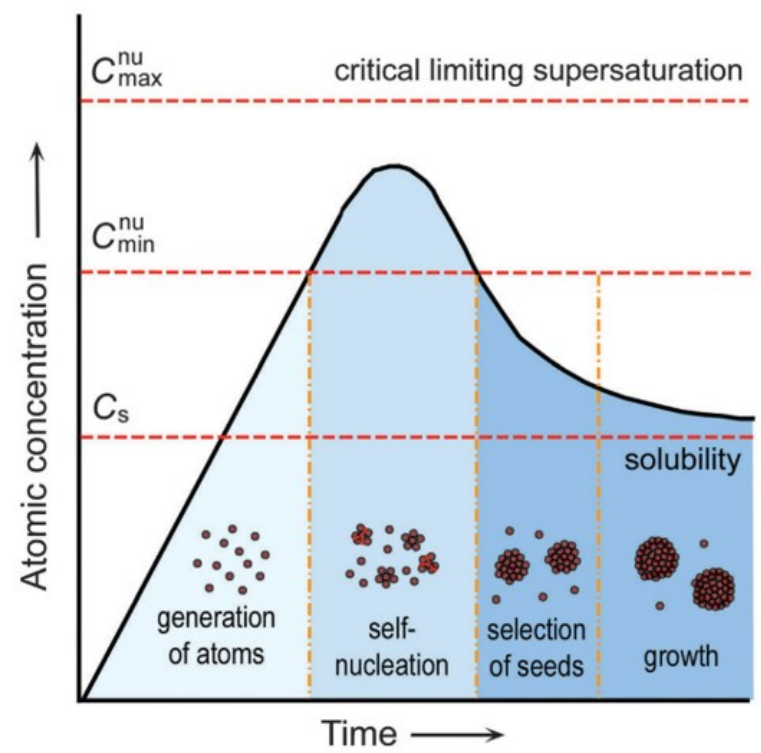

Figure 1.14: Modified LaMer model describing the inclusion of an extra step for selecting nuclei with appropriated crystal structures. Adapted with permission from (114). Copyright Royal Chemistry Society 2013.

As the silver nuclei clusters grow, fluctuations in their structure grow increasingly energetically costly causing them to stabilize in one of three dominant structures: single crystalline, singly twinned and multiply twinned. ${ }^{19,94,111,112}$ All three of these seed shapes co-exits in a typical silver nitrate/EG polyol synthesis, however, only the single crystalline seeds will lead to the formation of the desired nanocubes used in this study, as can be seen in Figure 1.15 below. ${ }^{19}$ 


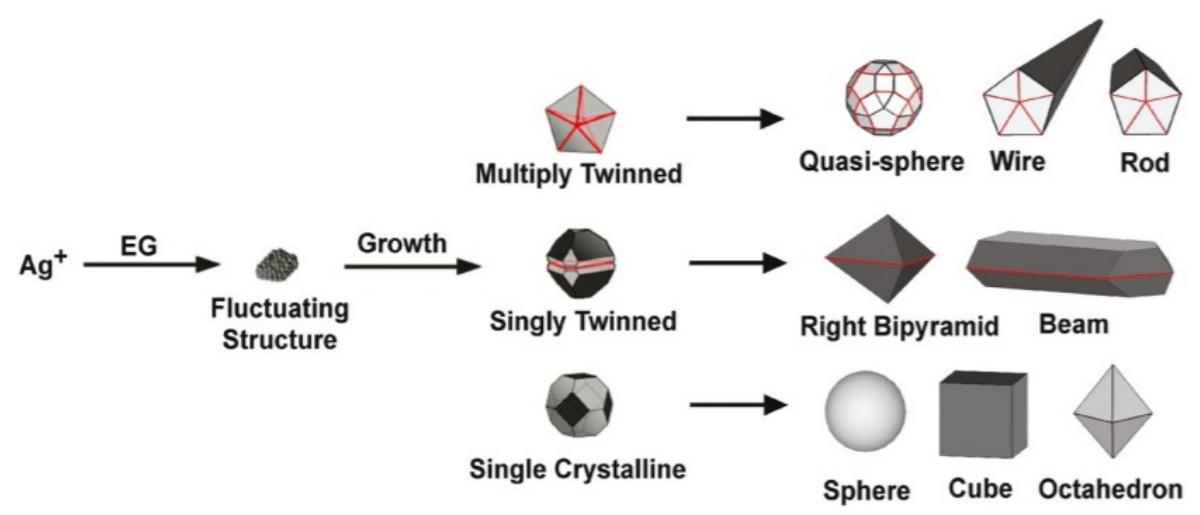

Figure 1.15: Possible reaction paths in the polyol synthesis of Ag nanostructures. Initial nuclei formed after the reduction of $\mathrm{Ag}^{+}$may fluctuate between different morphologies until growth to a critical size at which their morphology is locked into a single crystalline, singly twinned, or multiply twinned seed. The resultant respectively shaped seeds may grow into many different morphologies depending on choice of structure directing agent and presence of certain trace ions. Adapted with permission from (19). Copyright American Chemical Society 2011.

There are two steps to controlling a polyol synthesis of Ag nanostructures to ensure it results in the formation of silver nanocubes. The first is to manipulate reaction conditions so that it is only the single crystalline seed morphology that is encouraged and the second is to use structure-directing agents to ensure that the desire crystal facet of the silver is stabilized. The former is accomplished by addition of trace ions for oxidative etching and/or kinetic control and the latter by selection of a specific capping agent. ${ }^{19,94,111,112}$

\subsubsection{Seed Selection Through Oxidative Etching}

Oxidative etching is a common method used to control the shape of noble metal nanocrystals. It was first discovered by Xia et al in 2004 who demonstrated that the presence of select trace ions resulted in significant changes to the crystallinity and morphology of silver nanoparticles synthesized using the EG polyol method. ${ }^{17,113,114,119-122}$ What Xia et al found was that by adding $\mathrm{NaCl}$ to their EG polyol reaction they were able to preferentially etch the twinned seeds, leaving only the single crystalline seeds. ${ }^{117}$ This in turn 
led to the formation of only truncated nanocubes and tetrahedrons instead of the wide variety of shapes (wires, rods etc) typically produced by the EG polyol method. ${ }^{94,117}$ In the presence of $\mathrm{O}_{2}$ the $\mathrm{Cl}^{\text {- ions from }}$ the $\mathrm{NaCl}$ form $\mathrm{Cl} / \mathrm{O}_{2}$ oxidative pairs which oxidize the zero valent $\mathrm{Ag}^{0}$ nuclei back to $\mathrm{Ag}^{+}$ions, essentially dissolving the twinned seeds. ${ }^{118}$ If the introduction of the silver precursor is carefully controlled (such as in this experiment via the use of a syringe pump), the oxidative etching/dissolution process occurs at the same rate as the seed formation, limiting the morphology of seed available for growth. ${ }^{117-119}$

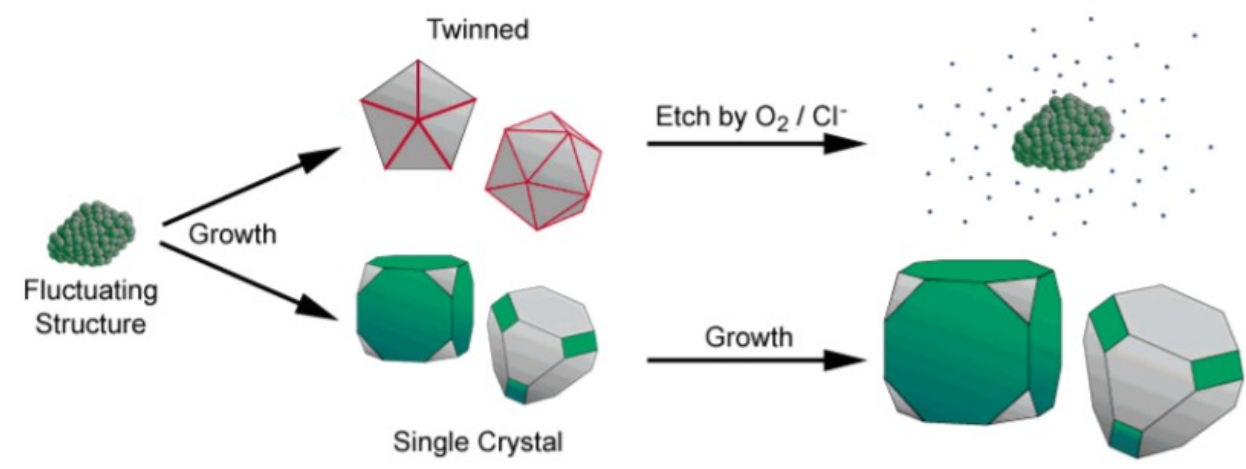

Figure 1.16: Mechanism of seed selection through oxidative etching. Adapted with permission from (114). Copyright Royal Chemistry Society 2013.

The reason why the twinned seed morphologies are etched and the single crystal seeds are not is because the former have an increased number of twin defects that result from structural strain. ${ }^{17,113,114,119-122}$ Multiply twinned seeds have a decahedron structure that is composed of five single crystal tetrahedrons oriented radially about a central axis. ${ }^{19,112}$ In this orientation the five tetrahedrons each have an interior angle of $70.5^{\circ}$ which leads to a $7.5^{\circ}$ gap. To make up for this gap the lattice distorts, forming an unstable structure that has multiple twinned defects (hence the name "multiply twinned"). Singly twinned seeds, as one can infer from the name, have a structure that has one twinned defect. The decahedron structure of multiply twinned seeds and a TEM image of an example of a resulting structural defect can be seen in Figure 1.17. 
a)

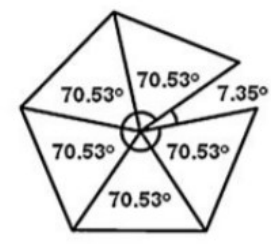

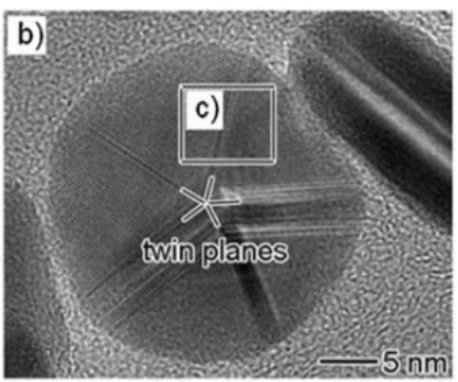

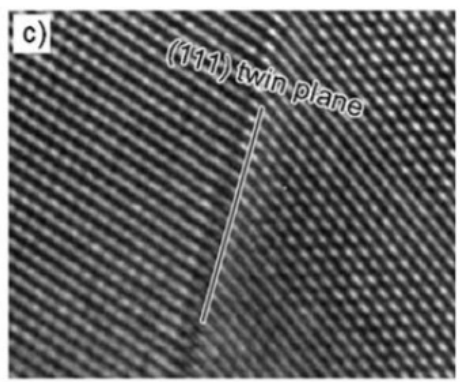

Figure 1.17: a) Schematic drawing of the decahedron structure of multiple twinned seeds b) High resolution TEM of Ag multiple twinned seed showing c) distorted boundary region that results from structural strain. Adapted with permission from (112). Copyright Wiley-VCH 2009.

Though $\mathrm{Cl}^{-}$ions are the most common ions used to influence the relative proportions of different $\mathrm{Ag}$ seeds for shape-directed growth of silver nanocubes, other ions can be used to achieve different shapes. ${ }^{19,112,120}$ $\mathrm{Br}^{-}$ions can also combine with $\mathrm{O}_{2}$ to form an oxidative etching pair; however, because $\mathrm{Br}^{-}$is a less corrosive anion it is only capable of dissolving multiply twinned seeds. ${ }^{19,112}$ Instead of preferentially selecting single crystalline seeds by preferentially etching away the other seed morphologies, $\mathrm{Br}^{-}$will only preferentially etch multiply twinned seeds leading to an accumulation of single crystalline and singly twinned seeds. While this is unhelpful in the context of synthesizing AgNCs, preferential etching using $\mathrm{Br}^{-}$can be used to direct the synthesis of right bipyramids which are the dominate structure produced from singly twinned seeds. ${ }^{120}$

\subsubsection{Seed Selection Through Kinetic Control}

Another way in which to control seed selection for an EG polyol synthesis is by controlling the reaction kinetics. ${ }^{121-123}$ Just like oxidative etching, kinetic control can be achieved by the addition of trace ions to the reaction solution. ${ }^{19,111,121-123}$ Siekkinen et al discovered that adding trace amounts of sodium sulfide or sodium hydrosulfide (both sources of $\mathrm{S}^{2-}$ ions) drastically reduced the reaction time of a classic $\mathrm{AgNO}_{3}$ EG polyol synthesis. ${ }^{122}$ Experimental evident demonstrates that $\mathrm{S}^{2-}$ reacts quite strongly with $\mathrm{Ag}$, even in 
very small trace amounts. ${ }^{122}$ In the case of $\mathrm{AgNO}_{3}$ polyol reactions, sulfur ions added to the solution react with $\mathrm{Ag}^{+}$to produce $\mathrm{Ag}_{2} \mathrm{~S}$ nanocrystallites. These $\mathrm{Ag}_{2} \mathrm{~S}$ nanocrystallites speed up the reaction in two ways: they provide sites for further growth of the silver nanocrystal and they catalyze the reduction of $\mathrm{Ag}^{+} .122$ Singly twinned and multiply twinned seed crystals grow more slowly than single crystalline seeds due to their structurally strained morphology. This means that when the speed of the reaction is increased due to the presence of $\mathrm{S}^{2-}$, single crystalline seeds are preferentially produced and AgNCs are the resulting final nanostructure. ${ }^{122}$ The seed selection through kinetic control is so effective that a high yield of AgNCs can be achieved even without the use of an oxidative etchant. ${ }^{122}$ The increased kinetics of the reaction also leads to a more uniform size distribution of particles. ${ }^{122}$

One of the factors that influences the size of a nanoparticle is the duration of its growth stage: the longer a nanoparticle is allowed to grow following its nucleation, the bigger it will get. A large size distribution in a nanoparticle array can be the result of varying growth times that result from different points of nucleation. When the onset of nucleation is accelerated, more nucleation events occur simultaneously, meaning that a higher number of nanoparticles will have the same growth time and the same resulting size.

\subsubsection{Shape Controlled Growth Through Capping Agent Selection}

All nanoparticles require some type of surfactant or capping agent that will prevent them from aggregating. One of the most common capping agents used for nanoparticles is polyvinylpyrrolidone (PVP). ${ }^{124-126}$ PVP is a bulky polymer consisting of a pyrrolidone ring with an alkyl functional group. ${ }^{124,125}$ Its structure can be seen in Figure 1.18 below. 


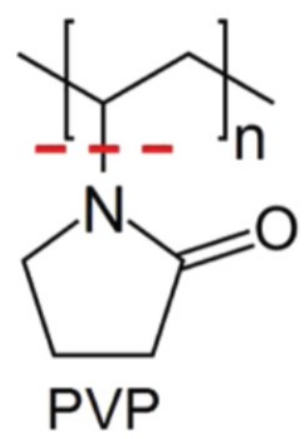

Figure 1.18: Structure of Polyvinylpyrrolidone. Dashed red line indicates the bond between the two constituent components. Adapted with permission from (124). Copyright American Chemical Society 2012.

PVP prevents aggregation of nanoparticles due to the repulsive forces between its hydrophobic carbon chains. The longer the PVP chains the greater protection they provide to the nanoparticle core and the more stable the suspension of nanoparticless. ${ }^{125}$ PVP not only prevents aggregation of nanoparticles, but it also helps directs their growth by promoting or hindering growth on different crystal faces. ${ }^{125}$

The face-centered cubic crystal structure of $\mathrm{Ag}$ has three different crystal facets: $\{100\},\{110\}$ and $\{111\}$. Growth of an Ag crystal occurs when an incoming metal atom binds to one of its crystal facets and occurs primarily on the $\{100\}$ and $\{111\}$ facets. PVP binds most strongly to the $\{100\}$ face of Ag due to the strong Van der Waal's attraction forces between the ethane component of the PVP and the Ag and the direct binding of the oxygen atom. ${ }^{124}$ When PVP binds to the $\{100\}$ facet it passivates it, preventing the addition of additional metal atoms. ${ }^{94,125-127}$ The $\{111\}$ facets on the other hand are uncovered and available for continuous growth. Incoming Ag nuclei form adatoms on the $\{111\}$ that then migrate to the face edge. The migration of adatoms to the face edge elongates the $\{100\}$ facets. Depending on the morphology of the seed crystal, this elongation results in different shapes. For multiply twinned crystals elongation of the $\{100\}$ facets results in the formation of nanowires but for single crystalline shaped seeds (which are the seeds preferentially selected in this synthesis) elongation of the $\{100\}$ facets results in the formation results in nanocubes with side length $>25 \mathrm{~nm} .{ }^{95}$ 


\subsection{Polymer Thin films}

Polymers are macromolecules made up of repeating subunits. They are characterized as crystalline or amorphous depending on how ordered their molecular structure is. ${ }^{128}$ Crystalline polymers have distinct melting temperatures, but amorphous polymers have what is known as a glass transition temperature (Tg). ${ }^{129}$ Below Tg a bulk polymer will be brittle and behave similar to a glass, but above $\mathrm{Tg}$ the bulk polymer will behave like a rubbery or viscous fluid. ${ }^{129}$

When the thickness of a polymer is confined to a thin film (100 nm or less) it will have a different Tg than it would in bulk. ${ }^{129-131}$ The model most commonly used to explain this is the three-layer model which divides polymer thin films into three distinct layers based on their proximity to the substrate and air respectively. ${ }^{129-131}$ At the top layer, located at the polymer-air interface, the polymer behaves in a mobile, liquid-like fashion. ${ }^{131,132}$ Because this layer has no bordering polymer, it will have lower mass density, making it easier for polymer rearrangement. ${ }^{132}$ The intermediate layer possesses bulk like properties; as the film thickness decreases so does the thickness of this layer. ${ }^{133}$ The bottom layer, located at the polymer-substrate interface, will have greater local density compared to the top and middle layers. ${ }^{133}$ This localized density depends on the attractive interactions between the polymer and substrate and will increase proportionally. ${ }^{133}$

One polymer frequently used for thin films is polystyrene (PS), the structure of which can be seen in Figure 1.19 below. Polystyrene is the polymer of choice used as the supporting thin film for the AgNCs in this study as it makes very smooth films, it has a good affinity for the AgNCs, and it has a relatively low $\operatorname{Tg}$ that is still above room temperature. ${ }^{134}$ 


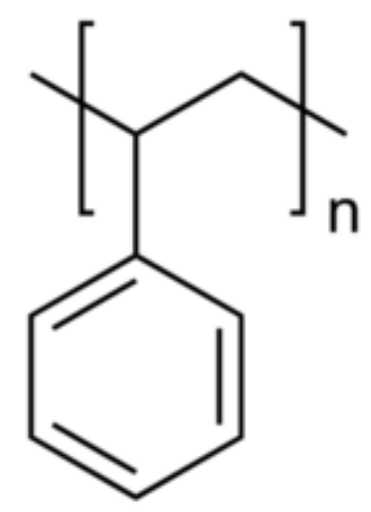

Figure 1.19: Molecular structure of Polystyrene. ${ }^{135}$

Bulk polystyrene has a $\mathrm{Tg}$ of $~ 100{ }^{\circ} \mathrm{C}$ but $\mathrm{Tg}$ values of polystyrene thin films ranging from $75-107^{\circ} \mathrm{C}$ have been reported. ${ }^{132,133}$ The exact reason for discrepancies between experimentally measured $\mathrm{Tg}$ values of PS thin films and the Tg of bulk polystyrene are not confirmed, but studies suggest that the film thickness, thickness of the mobile surface layer and heat flow processes can all play a role in raising or lower the $\mathrm{Tg}$ of PS thin films. ${ }^{132,133}$

\subsubsection{Nanoparticle Polymer Thin-Film Composites}

The supported $\mathrm{AgNC} /$ polystyrene substrates used in this study are a nanocomposite material. Some common configurations of metal nanoparticle/polymer composites can be seen in Figure 1.20 below. 

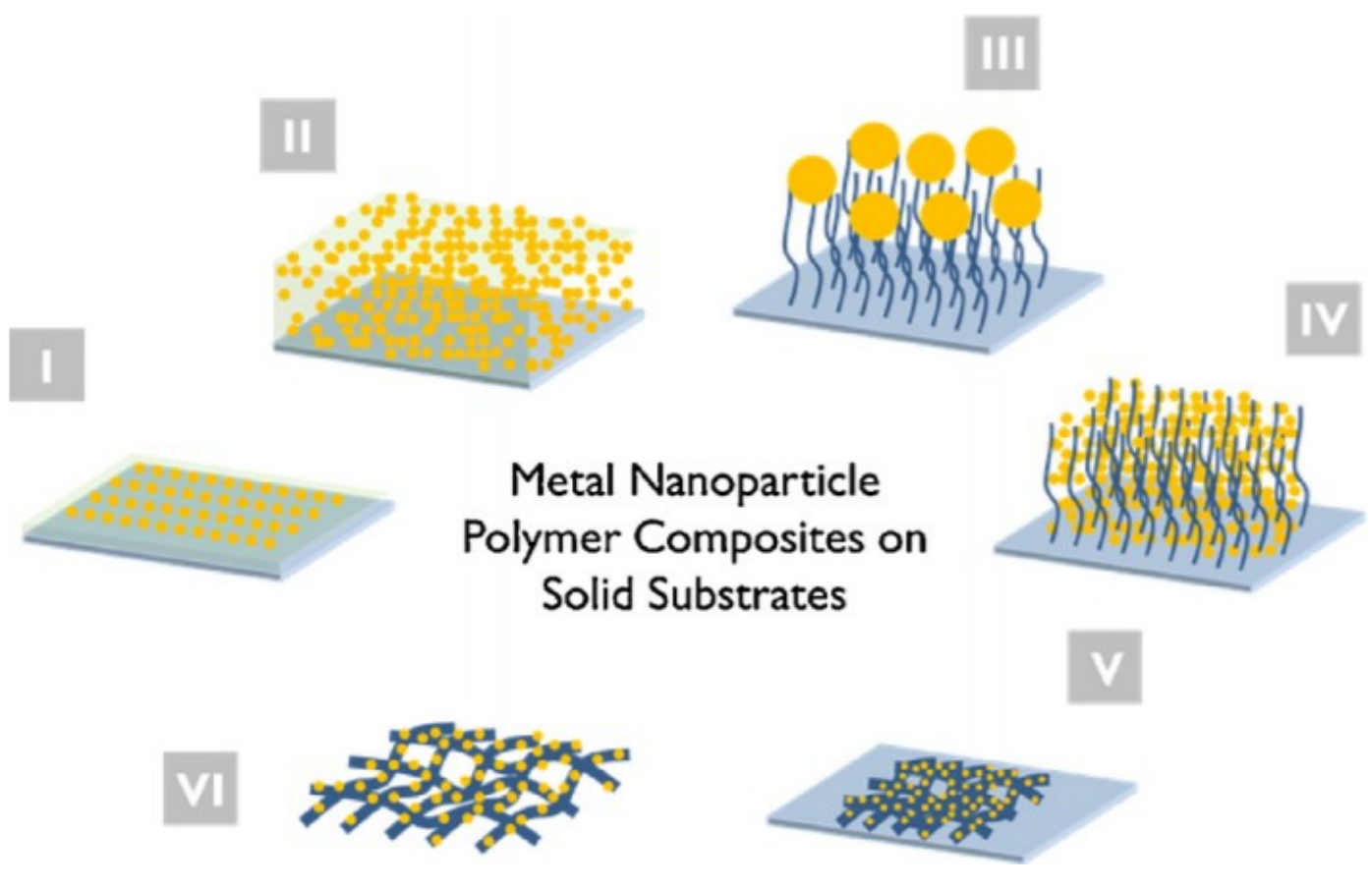

Figure 1.20: Several general configurations of metal nanoparticle polymer composites discussed in this review, including nanoparticles (I) immobilized on a thin polymeric film adsorbed or spin-coated on a substrate, (II) three-dimensionally distributed within a polymer gel matrix, (III) adsorbed on polymer brush grafted-to or -from the substrate, (IV) infiltrated within the brush, (V) decorated on polymer fibers electrospun on the substrate and (VI) decorated on free-standing polymer fibers. Reproduced with permission from (136). Copyright Elsevier 2016.

Nanoparticle/polymer composites have numerous applications in catalysis, sensing, electronics, and energy harvesting and storage. ${ }^{136-138}$ The nanoparticle/polymer composites used in this study fall into the category of metal nanoparticles immobilized (or supported) on a thin polymeric film that has been spincoated on a substrate (Figure 1.20I). While this configuration is commonly used for field-enhanced spectroscopies, catalysis, and sensing, it also provides an avenue for the synthesis of other types of nanoparticle/composites in which nanoparticles are partially or fully embedded in a polymer film. ${ }^{139-141}$ Integration of metal nanostructures into a conventional polymer matrix can dramatically increase the polymer's mechanical strength, electrical and thermal conductivity, and impart unique optical or antibacterial character to select polymers. ${ }^{137-139,142-144}$ One way to synthesize these types of 
nanocomposites is by embedding nanoparticles in to a polymer thin-film on which they have been deposited.

\subsubsection{Embedment of Nanoparticles in Polymer Thin-Films}

In order for a nanoparticle to embed in a polymer thin film two requirements need to be satisfied. ${ }^{139-141,145}$ The first requirement is a kinetic requirement that dictates that nanoparticles on the surface of a polymer thin film will embed when the polymer is heated beyond its glass transition temperature. ${ }^{140,141}$ The second requirement is a surface energy requirement that can be modelled using the Helmholtz free energy of the polymer and the nanoparticle. ${ }^{37}$

According to Kovacs and Vincent, the thermodynamic driving force behind embedment is a decrease in free energy. ${ }^{146}$ The Helmholtz surface free energy of a single rigid particle on a flexible polymer can be modelled by the equation

$$
\Delta A_{S}=a\left(\gamma_{p s}-\gamma_{p}\right)
$$

where $a$ is the surface area of the particle, $\gamma_{p}$ is the surface tension of the particle, $\gamma_{p s}$ is the interfacial surface tension between the particle and the polymer surface, and $\Delta A_{S}$ is the Helmholtz surface free energy..$^{146}$

Supposing that $\mathrm{T}>\mathrm{Tg}$, embedment will begin to occur if $\Delta A_{S}<0$, satisfying the equality $\gamma_{p}>\gamma_{p s}{ }^{146}$ It is important to note that the inequality $\gamma_{p}>\gamma_{p s}$ only applies to the requirements that a particle needs to begin embedding. In order for the particle to become fully incorporated into the polymer surface, $\gamma_{p}>$ $\gamma_{p s}+\gamma_{s} .{ }^{146}$ If $\gamma_{p s}+\gamma_{s}>\gamma_{p}>\gamma_{p s}$ then only partial embedment of the cube will occur.

The equations presented above apply to bare nanoparticles on a polymer surface. The AgNCs used in these investigations are capped with a layer of PVP meaning that the surface energy of the nanoparticle in the above equations $\left(\gamma_{p}\right)$ should be substituted with the surface energy of PVP $\left(\gamma_{p v p}\right)$. The surface 
energy of PVP is $\sim 43 \mathrm{~mJ} / \mathrm{m}^{2}$ and the surface energy of polystyrene is $33 \mathrm{~mJ} / \mathrm{m}^{2} .{ }^{147,148}$ Because the condition of $\gamma_{p v p}>\gamma_{p s}$ is satisfied, the capped particles will embed when the polystyrene is heated above its Tg.

The reason why nanoparticles on the surface of a polymer can embed when the polymer is heated above $\mathrm{Tg}$ is because the polymer chains become increasingly mobile, to the extent where they will flow around the particle enabling embedment. ${ }^{139,141,145}$ The rate at which embedment occurs depends on the temperature at which the polymer film is heated. The higher the temperature the more mobile the polymer chains and hence the more quickly the particle will embed.

Embedment of plasmonic nanoparticles into their supporting polymer thin film can be accomplished using two techniques: conventional heating or thermoplasmonic heating. ${ }^{84,139-141,145,149}$ Conventional heating methods involve placing the polymer nanoparticle composite on a heat source and heating the substrate to the glass transition temperature. Thermoplasmonic embedment involves using lasers set at appropriate wavelengths to excite plasmonic nanoparticles until they have reached the $\mathrm{Tg}$ of the polymer and begin to embed. While the conventional heating technique requires less costly and complex equipment and allows for the careful monitoring of embedment depth, it cannot be used for spatially resolved or selective embedment unless specifically designed polymer frameworks are used. ${ }^{139,141,145}$ Thermoplasmonic heating on the other hand can be performed with more localized precision and spatial resolution but typically requires sophisticated laser equipment. ${ }^{150}$ It is also more challenging to control and can result in substrate damage. ${ }^{84,149,150}$ The purpose of this investigation it to develop a thermoplasmonic embedment technique that can achieve spatial resolved embedding with simple equipment and minimal sample damage.

\subsection{Langmuir-Blodgett Method}

There are many different techniques which can be used to deposit nanoparticles on polymer thin films. ${ }^{136}$ Block co-polymer templating, laser ablation, metal-assisted chemical etching, self-assembly, and chemical vapour deposition can all be used to synthesize nanoparticle/polymer thin film substrates, however, these 
techniques can be time consuming and require costly equipment. The Langmuir Blodgett method in contrast is relatively efficient and requires less expensive equipment that is relatively easy to use. Though initially developed for depositing organic materials, Langmuir techniques have been very successful for depositing different shapes and sizes of nanostructure. ${ }^{151}$

The Langmuir method involves filling a shallow trough (known as a Langmuir trough) with water and suspending nanoparticles on the surface of the water. An image of the NIMA Langmuir trough used in this experiment with deposited nanoparticles can be seen in Figure 1.21 below.

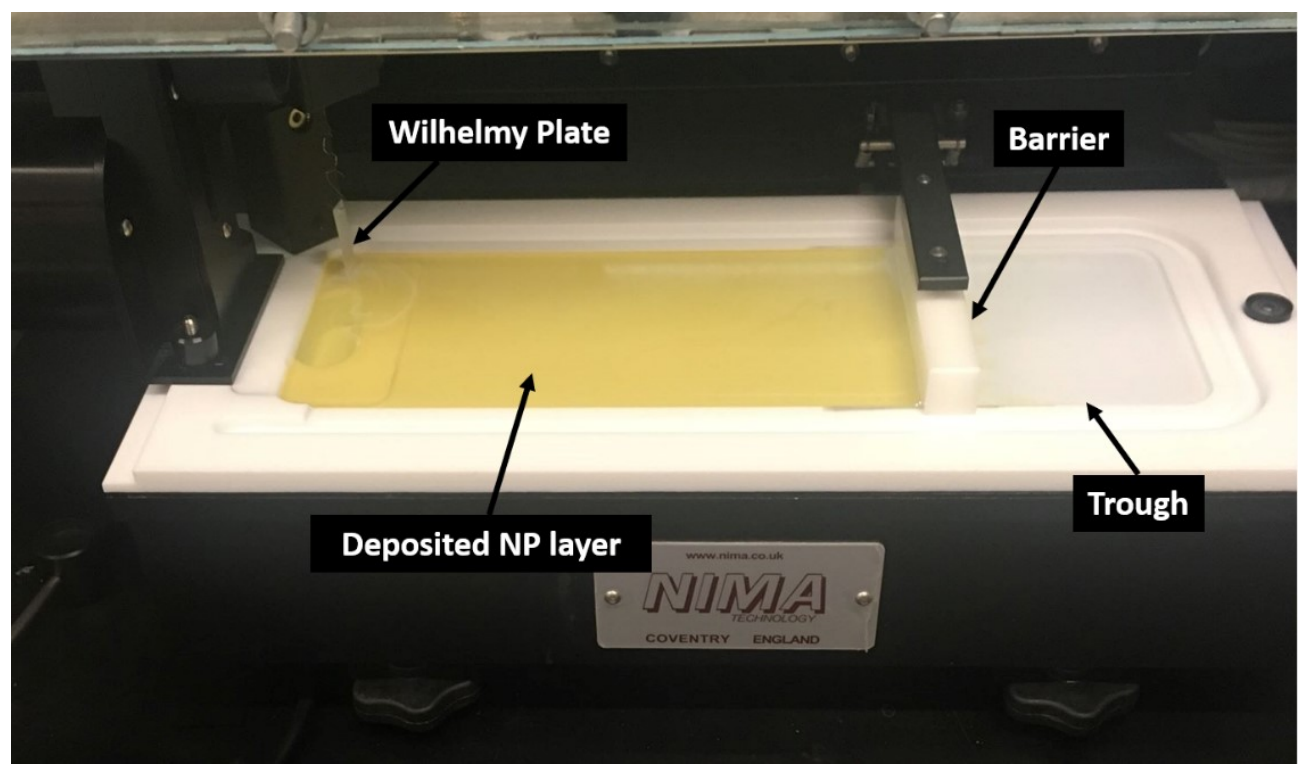

Figure 1.21: Photo of NIMA Langmuir-Schaefer Trough with Components Labelled.

Prior to being deposited on the water the nanoparticles are mixed with a volatile solvent and a lipid spacing molecule. The volatile solvent is chosen on the basis that it evaporates quickly following deposition and is not soluble in water (this is so that the nanoparticles are able to remain suspended on the water's surface). In this investigation chloroform was used as the volatile solvent and DOPC (1,2dioleoyl-sn-glycero-3- phosphocholine) was used as the lipid spacing molecule. Typically, the reason why the nanoparticles are able to stay suspended on the water's surface after being deposited dropwise are 
because of their hydrophobic capping agent. The PVP capped AgNCs made in this study are able to stay suspended on the water's surface due to the PVP's long carbon chain. ${ }^{125}$

How well spaced out the particles are on the surface depends on the amount of the spacing molecule used and the amount of the solution that is deposited. The more solution that is deposited the greater the density of the nanoparticles. Additional control over surface density can be accomplished by the moveable barrier which can open and close, decreasing and increasing surface density and pressure respectively.

\subsubsection{Measuring Surface Pressure}

The surface pressure of the trough is measured by the Wilhelmy plate (Figure 1.21) which is dipped in the surface of the trough. There are three forces acting on the Wilhelmy plate: gravity and surface tension which are acting on the plate in a downward direction, and buoyancy which is acting in an upwards direction. ${ }^{152}$ The total downward force experienced by the plate is the difference between the sum of the weight of the plate and the surface tension and the upwards force of water displaced by the plate. ${ }^{152}$ This is modelled by Equation 29 below

$$
F=\left[\left(\rho_{P} l w t\right) g+2(w+t)(S T) \cos \theta\right]-\left(\rho_{L} d w t\right) g
$$

where $l, w$, and $t$ are the length, width and height of the plate; $\rho_{L}$ and $\rho_{P}$ are the density of the liquid and the plate respectively; $S T$ is the surface tension; $g$ is the acceleration of gravity; and $\theta$ is the contact angle of the plate and liquid. ${ }^{152}$ The first term represents gravity, the second surface tension and the third buoyancy.

Zeroing the pressure before taking any isotherm measurements eliminates the first term from Equation 29. The balance that the plate is attached to maintains the plate at a constant level through changes in surface tension, eliminating the third term in the equation leaving 


$$
F=2(w+t)(S T) \cos \theta
$$

Because the plate and liquid contact angle is 0 , and $\cos (0)$ is 1 , the equation can be further simplified to

$$
F=2(w+t)(S T)
$$

which can be rearranged to express surface tension in terms of force:

$$
S T=\frac{F}{2(w+t)}
$$

Force is measured in $\mathrm{mN}$ and the length and height of the plate are in meters, giving $S T$ units of $\mathrm{mN} / \mathrm{m} .{ }^{152}$ The surface pressure of the nanocube monolayer is the sum of the surface pressure $\left(\mathrm{P}_{\text {surf }}\right)$ and the surface tension of the liquid in the trough, which remains constant at $-72.8 \mathrm{mN} / \mathrm{m}$ at STP conditions. ${ }^{152}$

$$
S T+P_{\text {surf }}=\text { Constant }
$$

The surface tension and pressure are controlled by the amount of nanoparticles deposited on the trough and the moveable barrier (Figure 1.23). The greater the quantity of nanoparticles deposited on the trough, the lower the surface tension and the higher the surface pressure. ${ }^{152}$ As the barrier closes (moves in the direction towards the Wilhelmy plate) it compressed the monolayer, lowering the surface tension and increasing the surface pressure. Opening the barrier (moving it in the direction away from the Wilhelmy plate) increases the surface tension, decreasing the surface pressure.

Prior to depositing the nanocubes on the trough it must be ensured that there are no contaminates on the surface of the trough that may compromise the cube monolayer. To measure the cleanliness of the trough a pressure-area isotherm is taken, which measures the surface tension of the trough across its area. Assuming there are no contaminates (molecules, dust particles etc.) on the trough the pressure-area isotherm should be 0 across most of the length of the trough. Once it is confirmed that the trough is clean 
and the cubes are deposited, the process of transferring the monolayer from the trough to the desired substrate can begin.

\subsubsection{Transferring Monolayer to Substrate}

There are two different Langmuir deposition techniques that can be used to transfer the monolayer deposited on the trough to a substrate: Langmuir-Blodgett Deposition and Langmuir-Schaefer Deposition. In Langmuir-Blodgett (LB) deposition the substrate is immersed into the trough and lifted out vertically (Figure 1.23 left). In Langmuir-Schaefer (LS) deposition the substrate is dipped horizontally into the substrate along the water air interface (Figure 1.22 right). Langmuir-Blodgett is typically used for hydrophilic substrates while Langmuir-Schaefer is used for hydrophobic substrates. In this study the substrate of choice is a glass microscope slide with a PS thin film (hydrophobic), thus the Schaefer deposition technique is used.
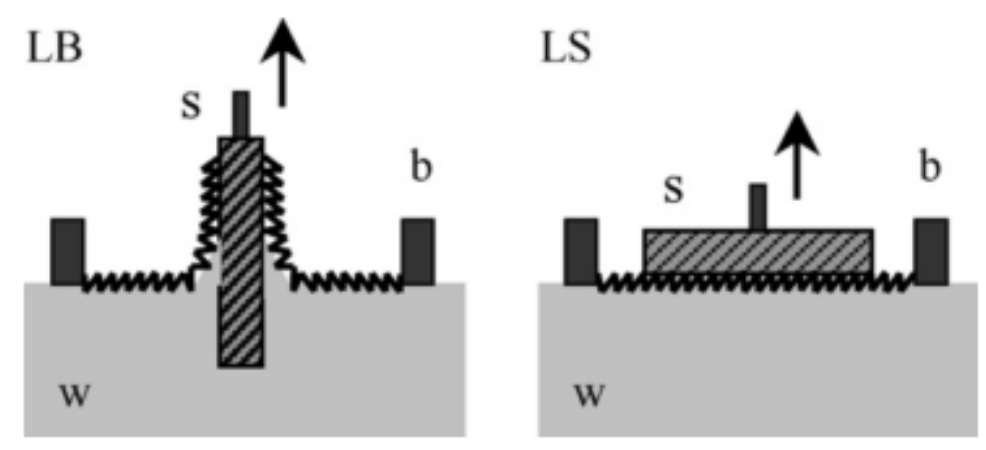

Figure 1.22: Blodgett deposition (left) and Schaefer deposition (right).Reproduced from (153). Copyright Elsevier 2006.

\subsection{Modelling of Plasmonic Systems}

Often when investigating the various properties of nanostructures it is necessary to conduct modelling simulations. Modelling simulations are based on computational calculations and provide valuable 
information that is difficult to determine otherwise. Simulations can help researchers determine optimal parameters for experiments and they allow researchers to compare their own experimental data to data predicted by theory. ${ }^{154,155}$ The most common computational methods used in plasmonic nanomaterials research are finite-difference time domain (FDTD) method, finite-element method (FEM), and discrete dipole approximation (DDA). FDTD, FEW and DDA use Gans theory (an extension of Mie theory for select shapes of nanoparticles) or Mie theory to calculate optical and electronic properties of different shaped nanomaterials. This work uses the FDTD method which is explained below.

\subsubsection{Finite Difference Time Domain Modelling Method}

The FDTD method, invented in 1961 by Dr. Kane S Yee, uses the temporal and spatial derivatives of Maxwell's equations to solve the various field vectors at discrete points in a cell that is located in a spatiotemporal grid. ${ }^{156-158}$ The form of Maxwell's equations used in FDTD can be seen below

$$
\begin{aligned}
\frac{\partial \vec{D}}{\partial t} & =\nabla \times \vec{H} \\
\vec{D}(\omega) & =\varepsilon_{0} \varepsilon_{r}(\omega) \vec{E}(\omega) \\
\nabla \times \vec{E} & =-\mu_{0} \frac{\partial \vec{H}}{\partial t}
\end{aligned}
$$

where $\vec{D}$ is displacement, $\vec{H}$ is magnetic field, $\vec{E}$ is electric field, $\varepsilon_{0}$ is permittivity (an electric field's ability to pass through a material), $\varepsilon_{r}(\omega)$ is the complex dielectric function, $\mu_{o}$ is the permeability (measure of a magnetic field's ability to pass through a material) and $\nabla x$ is the curl operator which acts on a field. ${ }^{149}$ The values of $\vec{D}, \vec{H}$ and $\vec{E}$ depend on the cartesian coordinates $(\mathrm{x}, \mathrm{y}, \mathrm{z})$ of a point in space and the time. ${ }^{155,156}$ FDTD solving software works by discretizing a simulation space using a 2D or 3D mesh grid and then solving the Maxwell equations in the subset spaces of the grid. ${ }^{155,156}$ For materials like metals, whose electronic and magnetic fields change quickly, fine mesh $(0.5-1.0 \mathrm{~nm})$ is required to ensure accuracy of computed 
results. Unfortunately, the finer the mesh used in FDTD calculations the more computing power that is required. While commercially available FDTD softwares, such as the Lumerical program used in these studies, are capable of making FDTD calculations for very small assemblies of metal nanostructures using an average desktop computer, more powerful programs and computers are required to conduct simulations for larger nanoassemblies.

To model the optical properties of nanostructures using FDTD software, different simulation objects are used to construct an approximation of the system of interest. These objects are usually selected from a library provided by the software and can be assigned different materials. An example of a simple system consisting of a dielectric film and a capped AgNC can be seen in Figure 1.23 below.

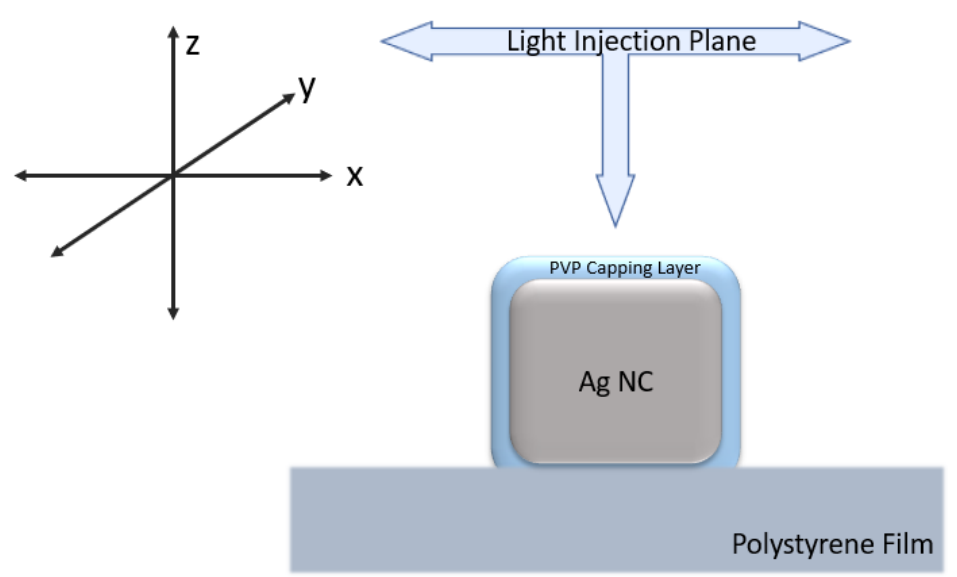

Figure 1.23: Scheme of Basic Object Layout in FDTD Model.

The software uses semi-empirical dielectric functions (a model of the dielectric functions fitted to experimental data) to predict how the system will interact with the chosen light sources. By varying shape, size, material, dielectric environment, and direction and type of incident light one can use FDTD simulations to determine how the optical and electronic properties of a nanostructure are affected by 
different variables. While the example shown in the Figure 1.23 is simple, FDTD simulations can be used to model many different shapes of metal and semiconductor nanoparticles, as well as larger assemblies of nanostructures. In this work FDTD is used to model the absorption cross section and near-field electric field enhancement factor (EEF) of different sizes of individual AgNC and different sizes and shapes of AgNC clusters (AgClusters).

\subsubsection{Discrepancies Between Experimental Conditions and Modelled Conditions}

While numerical modelling is an excellent tool to use to determine various properties of different nanomaterials using theoretical calculations, there can be discrepancies between experimental results and those calculated by computational programs. ${ }^{159-162}$ Typically discrepancies between experimental and computation methods can be attributed to the fact that properties such as size distribution, geometric parameters and dielectric functions of the actual materials used may not be identical to values inputted or estimated by the softwares. ${ }^{159-162}$ Another parameter than can differ considerably between computational and experimental systems is the type of light source used. ${ }^{163}$

In these experiments the light source used to thermoplasmonically excite the AgNCs is a laser of unknown polarization. In the FDTD simulations conducted, the light source is polarized either $0^{\circ}$ or $90^{\circ}$, along the $x$ and $y$ axis of the respectively (Figure 1.24 shows an example with an AgNC dimer). 


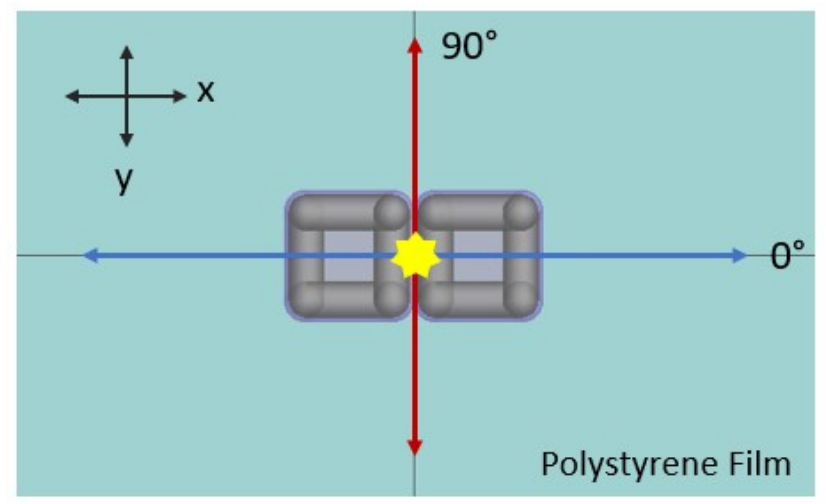

Figure 1.24: Illustration of the axes of polarization for the $0^{\circ}$ (blue arrow) and $90^{\circ}$ (red arrow) light used in FDTD calculations.

The yellow star in the centre of the two AgNCs represents the injection of the light along the z-axis.

The angle of light polarization does not alter the calculated absorption spectra of individual isotropic nanoparticles, however, it does affected the absorption spectra of nanoparticle clusters and dimers. ${ }^{48,163,164}$ This is because the polarization determines whether the transverse or longitudinal coupling mode of the particles will be excited. ${ }^{79}$ Typically, the transverse coupling mode of a nanoparticle dimer (the mode oriented perpendicular to the dimer axis) is far less significant that its longitudinal counterpart (the mode oriented along the coupling axis). However, when the E-field component of light is oriented along the same axis as the transverse mode, as is the case with the $90^{\circ}$ polarized light shown above, the coupling of the transverse mode becomes far more significant. This will not only alter the absorption properties but also the EFF of the cluster or dimer. ${ }^{79}$ An illustration of the longitudinal and coupling modes of an AgNC dimer on a PS thin film and the resulting direction of the Efield can be seen below. 

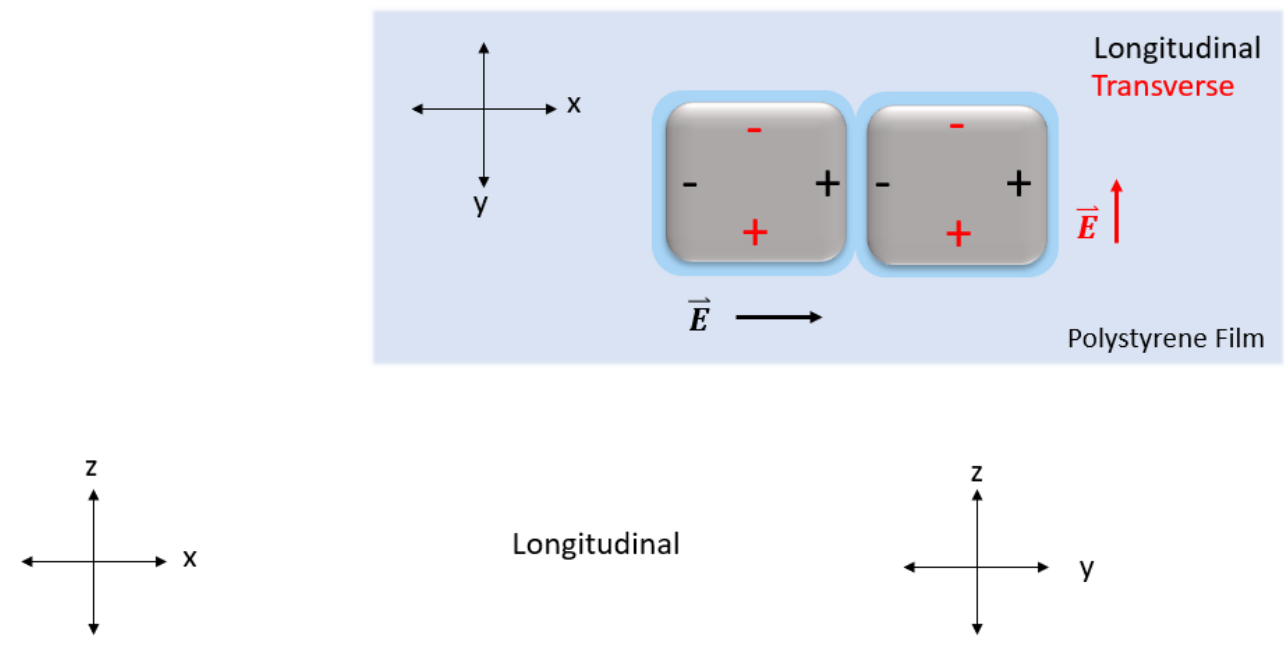

Longitudinal

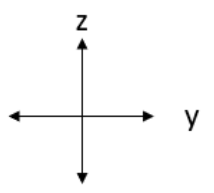

Transverse
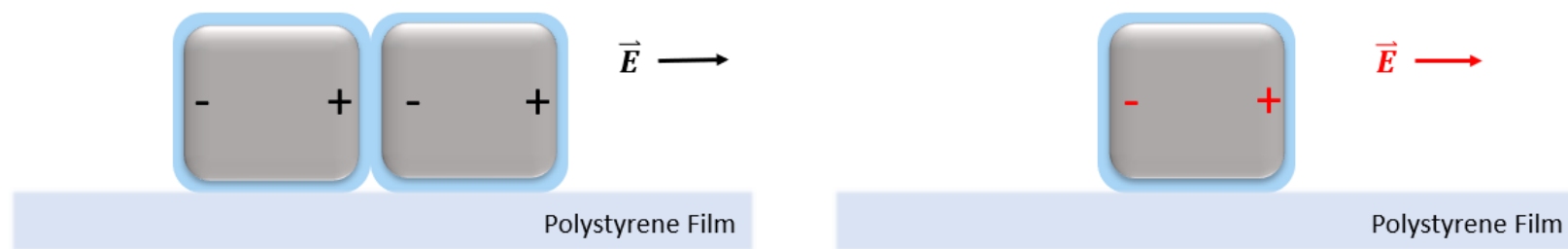

Figure 1.25: Schematic illustration of longitudinal (left) and transverse (right) coupling modes in an PVP capped AgNC dimer.

The top image illustrates both coupling modes on the dimer simultaneously.

Non-polarized light sources contain E-field components that are randomly polarized in all directions, and thus will excite both the transverse and longitudinal coupling modes in any AgNC dimers and clusters. ${ }^{165}$ The laser used in these experiments is initially polarized in a certain direction out of the source, but as it passes through the optical fiber used to re-route it to the sample the polarization changes so the polarization of the laser that reaches the sample is unknown. To most accurately compare our experimental results with those calculated using modelling, the absorption spectra and EEF for all applicable structures were calculated using both $0^{\circ}$ and $90^{\circ}$ polarized light.

\subsection{Thesis Objective}

The objective of this work is to investigate if selective thermoplasmonic heat generation can be used to achieved selective embedment in arrays of supported silver nanocubes. Previous studies by the lanoul group have documented thermoplasmonic heating in nanocrystal/polymer composite thin films and the 
selective embedment of AgNCs using different polymer thin film scaffolds and conventional heating methods; however, methods for selective embedment using varied thermoplasmonic excitation wavelengths have not yet been explored by our group. ${ }^{139,149}$ In this work, monolayers of silver nanocrystals containing randomly distributed silver nanocubes (AgNCs) and silver nanocube clusters (AgClusters) deposited on PS substrates were exposed to different wavelengths of lasers to determine if different wavelengths of laser could be used to selectively thermoplasmonically heat and embed the different structures. The hypotheses, based on thermoplasmonic and polymer thin film theory, is that the individual AgNCs should selectively embed at shorter wavelengths and the AgClusters should selectively embed at longer wavelengths. As the laser exposure time increases, the depth of embedment should also increase. If these experiments are successful it will be the first instance of selective thermoplasmonic heating of individual nanoparticle structures within an array.

\section{Experimental}

\subsection{Materials and Experimental Overview}

\subsubsection{Reagents and Equipment}

All reagents were purchased from Sigma-Aldrich (unless otherwise indicated) and used without further modification. Deionized water was obtained from a Milli-Q system $((18.2 \mathrm{M} \Omega \mathrm{cm}$, Millipore, Bedford, MA). Glass syringes, Luer-Lok syringe filters, small centrifuge tubes (1.5 mL and $2.0 \mathrm{mmL}), 15 \mathrm{~mL}$ plastic test tubes, 1.5 dram screw cap vials, $20 \mathrm{~mL}$ scintillation vials, disposable pipette tips, stir bars and glassware were purchased from various lab equipment manufacturing companies. All glassware was cleaned with aqua regia (3:1 $\mathrm{HCL}$ and $\mathrm{HNO}_{3}$ ) and water (tap, distilled and MilliQ) prior to use.

\subsubsection{Instrumentation}


UV-Vis analysis was performed with a Shimadzu UV-2450 UV-Vis spectrometer and the corresponding UV Probe 2.21 program. All centrifuging was performed using a Labnet PRISM microcentrifuge.

Atomic force microscopy (AFM) characterization was performed using a Ntegra (NT-MDT Spectrum Instruments, Russia) AFM in semi-contact mode in air in room temperature. The AFM used silicon cantilevers probes with reflective gold coatings $\left(k_{c}\right.$ of $0.35-6.1 \mathrm{~N} / \mathrm{m}$ and a resonant frequency between 47 - 150 KHz, NSG03 model, NT-MDT Spectrum Instruments). The instrument operated using Nova Software (Nova RC1 version 1.0.26.1443) and all AFM image processing/analysis was conducted using the same software.

\subsection{Synthesis of Silver Nanocubes}

\subsubsection{Synthesis of AgNC}

The AgNCs were synthesized based on a modified approach to a method developed by Skrabalak et al. ${ }^{166}$ The cubes were made by combining $~ 0.4 \mathrm{~g}$ of 55,000 MW polyvinylpyrrolidone (PVP) with $15 \mathrm{~mL}$ ethylene glycol anhydrous (EG) in a round bottom flask (with a PPTE coated stir bar) and heating the solution to $150{ }^{\circ} \mathrm{C}$ in a oil bath while stirring at $300 \mathrm{rpm}$. Once the solution had reached $150^{\circ} \mathrm{C}$, it was left to stir at $300 \mathrm{rpm}$ for one hour. While the PVP solution was stirring, the three other solutions were prepared. A $0.5 \mathrm{M} \mathrm{NaCl}$ solution was prepared by dissolving $~ 0.2808 \mathrm{~g}$ of $\mathrm{NaCl}$ (BioShop) in $10 \mathrm{~mL} \mathrm{EG}$ in a glass scintillation vial and sonicating and vortexing until dissolved. A $3 \mathrm{mM} \mathrm{Na}_{2} \mathrm{~S}$ solution was prepared by dissolving $\sim 0.0023 \mathrm{~g}$ of $\mathrm{Na}_{2} \mathrm{~S}$ (weighed out into a glass vial using a glass Pasteur pipette) in $10 \mathrm{~mL}$ EG. A 0.3 $\mathrm{M}$ solution of $\mathrm{AgNO}_{3}$ was prepared by dissolving $\sim 0.48 \mathrm{~g}$ of $\mathrm{AgNO}_{3}$ anhydrous in $10 \mathrm{~mL}$ EG. The vials containing both the $\mathrm{Na}_{2} \mathrm{~S}$ and $\mathrm{AgNO}_{3}$ were covered in black electric tape as the reagents are photosensitive. When the PVP solution had been stirred for 1 hour at a steady temperature of $150{ }^{\circ} \mathrm{C}, 25$ $\mu \mathrm{L}$ of the $\mathrm{NaCl}$ solution was added followed by $175 \mu \mathrm{L}$ of the $\mathrm{Na}_{2} \mathrm{~S}$ solution (both were dispensed using automatic pipettes). After these additions, the solution was allowed to stir for one minute. Next $1.5 \mathrm{~mL}$ 
of the $\mathrm{AgNO}_{3}$ solution was added at a rate of $0.5 \mathrm{~mL} / \mathrm{min}$ using a New Era automated syringe pump system. The reaction was continually monitored using a UV-Vis spectrometer by periodically taking small aliquots out of the reaction flask and dispersing in an ethanol-filled cuvette and taking the resulting UV-Vis spectrum. Once the spectrum indicated the presence of a peak at 350 nm (after about 15 minutes), an additional $1.5 \mathrm{~mL}$ of the $\mathrm{AgNO}_{3}$ solution was added at approximately $1 \mathrm{~mL} / \mathrm{min}$. When a peak at $\sim 460 \mathrm{~nm}$ became visible in the UV-Vis spectrum (after about 25 minutes), the reaction was removed from the heat and quenched in an ice bath.

\subsubsection{Cleaning and Storage of AgNC solution}

To remove unreacted reagents from the reaction solution the AgNC solution had to undergo cleaning steps prior to being stored. The AgNC solution was diluted with an equal volume of ethanol (EtOH) and divided into multiple $2 \mathrm{~mL}$ centrifuge tubes. The tubes were centrifuged at $12,000 \mathrm{RPM}$ for 30 minutes. The supernatant was then decanted from the tubes, $1 \mathrm{ml}$ of fresh $\mathrm{EtOH}$ was added to each tube and the cubes were redistributed in the fresh solvent using a vortex and sonicator. The solution was then concentrated into fresh $2 \mathrm{~mL}$ tubes and centrifuged again at 12,000 RPM this time for 10 minutes. The redistribution of the cubes and sonification was repeated three more times for a total of four times. Each time the cubes were redistributed in fresh solvent the volume of solvent added was decreased so that the total amount of centrifuge tubes required decreased by half until all of the AgNC in EtOH solutions had been concentrated into a single tube. This solution was stored in the fridge until further use.

\subsubsection{UV-Vis Characterization of AgNCs}

The first UV-Vis spectrum of the AgNC solution was taken immediately after synthesis and kept as reference. A UV-Vis spectrum was taken every 6-16 weeks to ensure that that the AgNC retained their desired shape and size distribution. EtOH was used as the baseline spectrum every time. 


\subsubsection{TEM Characterization of AgNC}

The AgNCs were analyzed using a FEI Tecnai G2 TEM Transmission Electron Microscope (TEM). The TEM samples were prepared by drop casting the solution onto a Copper-Carbon TEM grid (Electron Microscopy Sciences) and leaving to dry overnight so that the EtOH would evaporate. Two rounds of TEM imaging were conducted: one immediately after initial synthesis and one four months later to ensure that the cubes had retained their uniformity.

\subsection{Synthesis of Polystyrene Substrates}

\subsubsection{Cleaning of Slides}

Prior to being spin-coated with polystyrene microscope slides (Globe Scientific Inc) were cleaned.

First the microscope slides were immersed in piranha solution ( $10: 1 \mathrm{H}_{2} \mathrm{SO}_{4}$ and $\mathrm{H}_{2} \mathrm{O}_{2}$ ) for 10-20 minutes. Following the piranha, the slides were thoroughly rinsed with water and cut into three approximately equal sized sections using a diamond tipped pen. The cut slides were then immersed in piranha for an additional 10-20 minutes before being rinsed with water. The slides were then left to sit in water before being sonicated in methanol and dried with nitrogen. To minimize risk of contamination, the slides were only sonicated in methanol and dried immediately before being spin-coated with polystyrene.

\subsubsection{Preparation of Polystyrene Solution}

A $3 \%$ weight solution of polystyrene (PS) was made my stirring $\sim 0.25 \mathrm{~g}$ of $250,000 \mathrm{~g} / \mathrm{mol}$ PS (Acros Organics) in $\sim 9.5 \mathrm{~mL}$ toluene (Anachemie) in a glass scintillation vial at room temperature until completely dissolved (2-3 hours). Once the PS had fully dissolved in the toluene, the solution was passed through a $0.22 \mu \mathrm{m}$ polytetrafluoroethylene (PTFE) filter into a clean glass vial using a glass syringe.

\subsubsection{Spin Coating the Glass Slides with Polystyrene}


The slides were fixed one-by-one to the stage of a homemade spin coater using double sided tape. The 3\% PS solution was pipetted onto the slides until there was just enough of the solution to cover the surface of the slide. Once the surface of the slides was covered with the PS solution, the slides were spun at $4000 \mathrm{rpm}$ for 30 seconds -1 minute. They were then partially covered and left to dry at room temperature for 1 hour before being placed in an oven heated to $130^{\circ} \mathrm{C}$ overnight to anneal.

\subsubsection{Characterizing Surface Roughness and Film Thickness with AFM}

To analyze surface roughness several $512 \times 512$ point size images were taken of the PS films. Film thickness measurements were taken by scratching the surface of the slide with a clean razor blade and using the AFM and Nova Software to measure the depth of the scratch.

\subsection{Synthesis of Silver Nanocube Monolayers via Langmuir-Schaeffer Deposition}

\subsubsection{Cleaning the Langmuir-Schaeffer Trough}

First the Nima 311D Langmuir-Blodgett trough was cleaned using chloroform and surfactant-free Kimwipes. Next, the trough was filled with fresh MilliQ water and the water was removed with an aspirator. This process was repeated 2-3 times to remove impurities. Then a new Wilhelmy plate was added and the trough was filled with MilliQ water and left to stand for 20 minutes, before being emptied by the aspirator and refilled a final time. A pressure-area isotherm was conducted at a barrier speed of 30 $\mathrm{cm}^{2} / \mathrm{min}$ to determine the cleanliness of the trough. If the isotherm revealed a pressure equal to or less than $0.1 \mathrm{mN} / \mathrm{m}$, the trough was deemed clean enough to proceed with the deposition process. If not, the water-cleaning process was repeated until the desired pressure-area isotherm was achieved.

\subsubsection{Preparing the AgNC solution for Langmuir-Schaeffer Deposition}

The UV-Vis spectrum of a $1000 \mathrm{X}$ dilution of the AgNC solution was taken and based on the absorbance of the spectrum for the $1000 \mathrm{X}$ dilution, a $2 \mathrm{~mL}$ solution of AgNCs which would give a 
theoretical absorbance of 100-150 abs was prepared in EtOH. The AgNC solution was centrifuged at $12,000 \mathrm{rpm}$ for 10 minutes. The supernatant was then removed and the AgNCs were redispersed in chloroform and centrifuged at 10,000 rpm for 5-6 minutes. The chloroform supernatant was removed, the cubes were redispersed in $1.5 \mathrm{~mL}$ fresh chloroform, transferred to a fresh centrifuge tube and subsequently centrifuged for another 5-6 minutes at 10,000 rpm. This process was repeated twice for a total of three rinses in chloroform. After the third round, the cubes were redistributed in only $300 \mu \mathrm{L}$ of chloroform and $10 \mu \mathrm{L}$ of a $1 \mathrm{mg} / \mathrm{mL}$ solution of DOPC (Avanti Polar Lipids) in ethanol was added.

\subsubsection{Deposition of AgNC solution on Trough}

Using a 500uL glass syringe (one specifically designated for AgNC deposition which had been rinsed thoroughly with chloroform) the cube and lipid solution was carefully deposited on the trough. This was done by holding the syringe a few millimeters from the surface of the water and dispensing the cube solution in a controlled drop wise manner so that it dispersed on the surface of the water. Once enough of the cube solution had been dispensed so that the surface of the trough was a homogenous dark yellow (see Figure 1.21), it was left for 20 minutes so that the chloroform could evaporate. After the 20 minutes, a series of expansion/closing isotherm cycles were conducted to anneal the monolayer.

To deposit the AgNC monolayer onto the PS coated glass slides the slides were dipped or "tapped" horizontally onto the surface of the AgNC covered water and then dried under nitrogen. The trough's surface pressure was adjusted so that different samples were prepared with varying monolayer densities. The AgNC monolayers were investigated with UV-Vis spectroscopy and AFM.

\subsection{Characterization of AgNC Monolayer}

\subsubsection{UV-Vis of AgNC Monolayer Substrates}

The UV-Vis spectrum of each of the AgNC coated glass slides was taken using a PS coated glass slide as the baseline. The spectra of the individual AgNC coated glass slides were examined to determine 
whether there was a correlation between absorption and deposition pressure, as well as whether or not plasmon coupling had occurred.

\subsubsection{Characterization of Monolayer by Atomic Force Microscopy}

AFM was used to characterize the surface topography and particle density of the AgNC monolayers.

\subsection{Selective Embedment Laser Experiments}

\subsubsection{Description of Optical System}

The excitation experiments were done with a continuous wave (CW) $\mathrm{Ar} / \mathrm{Kr}$ laser (Coherent 70 series) through an inverted microscope (Olympus IX-71) with a 20X objective. The laser was set at varying power $(\mathrm{mW})$, current $(\mathrm{A})$ and wavelength for different experiments. Power was measured by a power meter located immediately before the focusing lens.

\subsubsection{Determining Embedment Threshold}

The first laser-exposure experiments were conducted to determine the embedment threshold of the cubes. The wavelength of the laser was held constant and the current, power, and exposure time was varied. AFM images of the AgNC monolayers were taken before and after laser exposure to determine whether or not embedment had occurred. Any holes that resulted from these preliminary experiments were imaged using AFM so that the various sets of holes could be compared with each other.

\subsubsection{Investigating Selective Embedment}

To perform the selective embedment experiments various wavelengths (458 nm and $568 \mathrm{~nm}$ ) and constant power (17 $\mathrm{mW}$ and $65 \mathrm{~mW}$ respectively) were used. The exposure time varied. The samples were imaged by AFM before and after laser exposure. Embedment was examined by comparing the AFM images 
before and after laser exposure and using various features in the Nova software to measure the topographic height of select features before and after laser exposure.

\subsection{FDTD Simulations}

Finite Difference Domain modelling was performed using a Lumerical Solutions Inc software program (Lumerical Solutions v8.15). A total-field scattered-field (TFSF) light source was used to calculate the absorption cross sections and E-field enhancement. The parameters used for the simulation settings had been previously verified using convergence testing. The simulation time was set to $100 \mathrm{fs}$ and a 800 $\mathrm{nm}$ cubic simulation space was used. The models consisted of silver nanocubes with edge lengths of either $30 \mathrm{~nm}, 45 \mathrm{~nm}$ or $60 \mathrm{~nm}$ with 15\% corner roundness and a $2 \mathrm{~nm}$ PVP capping layer, supported on a $150 \mathrm{~nm}$ thick PS film. The materials were described by an analytical model fitted to tabulated permittivity data of silver, polystyrene and PVP. A mesh override region extending at least $10 \mathrm{~nm}$ past each nanocube or structure with a $0.5 \mathrm{~nm}$ mesh size was used for all simulations. X-axis and y-axis symmetry boundary conditions (symmetric and antisymmetric) were used when applicable and adjusted depending on the angle of polarization. For the dimers and clusters the spectra were calculated at a light polarization angle of both 0 and 90 degrees. The absorption simulations they were conducted from $300 \mathrm{~nm}-900 \mathrm{~nm}$ with a total of 301 point collected.

\section{Results and Discussion}

\subsection{Sample Preparation}

This section provides an overview of the procedures used for the preparation of the supported AgNC substrates. Because thermoplasmonic heating depends directly on the optical characteristics of the nanoparticles in question, high quality supported AgNC monolayer samples had to be prepared. With the exception of the nanocube synthesis, each of these procedures was performed multiple times during the course of this study to generate fresh samples as needed. Representative results of the PS thin film 
synthesis and the deposition of the AgNCs on the PS thin films via Langmuir-Schaefer deposition are used to illustrate the techniques and analyses performed.

\subsubsection{Silver Nanocube Synthesis}

Extinction spectra of the AgNC solution were taken at different times during the polyol synthesis (Figure 3.1). The spectrum taken 10 minutes into the synthesis had a sharp dipolar peak at $403 \mathrm{~nm}$. As the reaction progressed, this peak red-shifted and broadened. At 15 minutes a quadrupolar peak at 350 $\mathrm{nm}$ appeared indicating that the particles were developing a more cubic shape. After 25 minutes the reaction was quenched as the dipolar and quadrupolar peaks had sufficiently red-shifted and a multipolar peak at $350 \mathrm{~nm}$ emerged, indicating the presence of AgNCs with edge length approximately $30-60 \mathrm{~nm} \cdot{ }^{33,167}$ The final peak positions of the dipolar, quadrupolar and multipolar modes were $460 \mathrm{~nm}$, $388 \mathrm{~nm}$, and $346 \mathrm{~nm}$ respectively.

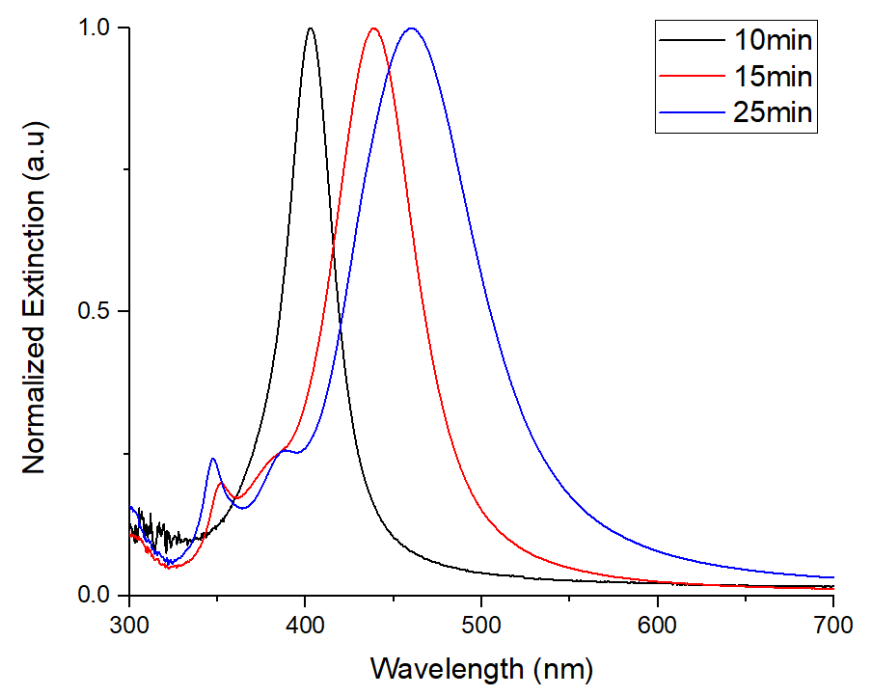

Figure 3.1: Extinction Spectra of AgNC in Solution at Different Growth Times. 
Transmission electron microscopy (TEM) was used to determine the shape and size distribution of the AgNCs. A total of 9 TEM images similar to Figure 3.2B and $\mathrm{C}$ were used to confirm the size and shape of the cubes. Figure 3.2D is a histogram that indicates the size distribution of 162 cubes whose edge lengths were measured using the TEM software. The mean cube size was calculated to be $49 \mathrm{~nm} \pm 9 \mathrm{~nm}$, which is in keeping with the size estimated from the extinction spectra. Because the resulting nanocrystals had a well-defined geometry and reasonable size distribution (Figure 3.2), the colloidal synthesis was concluded to be successful.
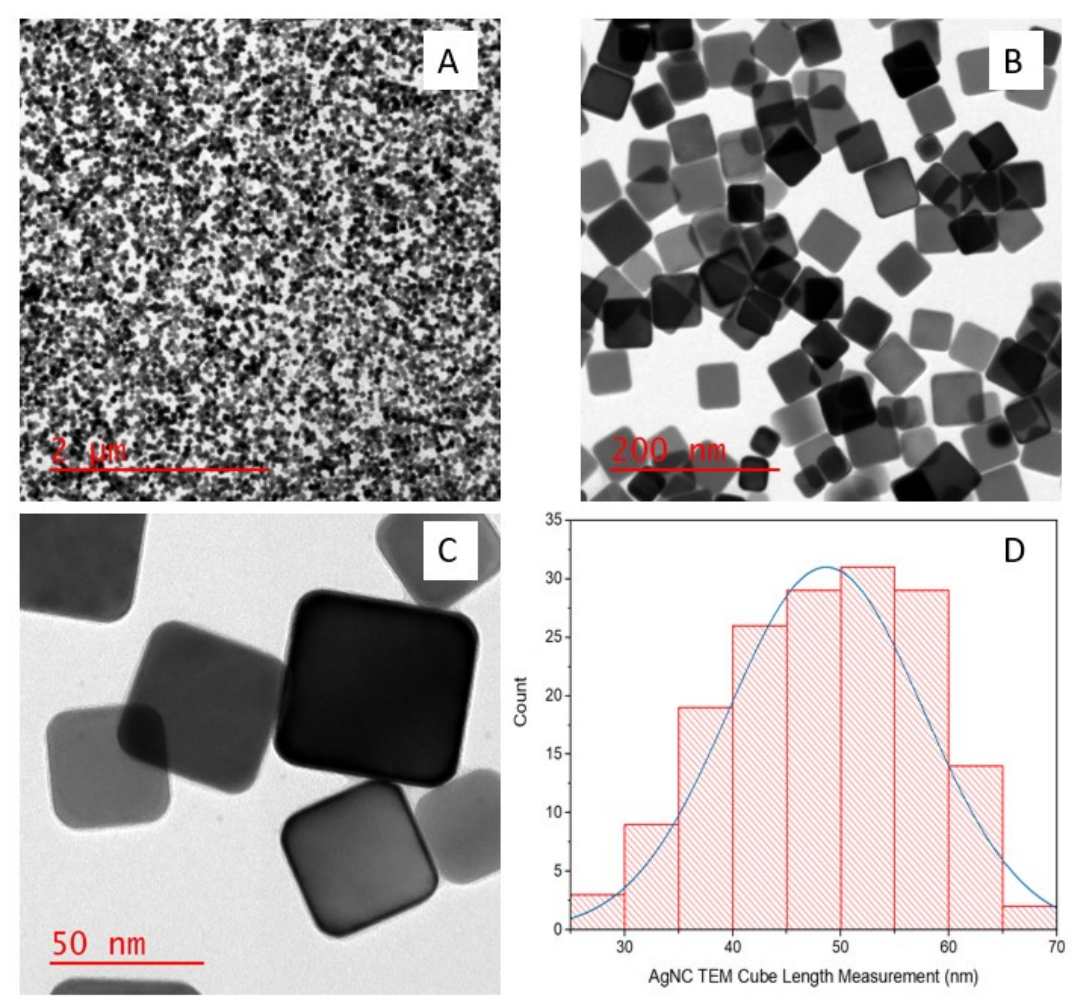

Figure 3.2: A) Large Scale TEM image of AgNCs B) Medium Scale TEM image of AgNCs C) Small Scale TEM image of AgNCs D) Histogram of AgNC Edge Length Measurements taken with TEM Software.

One of the primary challenges faced in nanomaterial synthesis is the synthesis of nanomaterials that retain their stability over time. ${ }^{109,168}$ Because the AgNC produced in this initial synthesis were used for all subsequent experiments over the course of 15 months, it was important to determine that the suspension 
remained stable and minimal Ostwald Ripening occurred. Ostwald ripening results in an increase in the average size of cubes, and thus would cause a red-shift in the dipolar mode in the extinction spectrum. ${ }^{169}$ Figure 3.3 shows the extinction spectra of the AgNC solution taken 4 months and 1 year following the initial synthesis. Despite the passage of time, the three spectra are essentially identical, indicating that the size and shape of the AgNCs were unchanged over the course of storage.

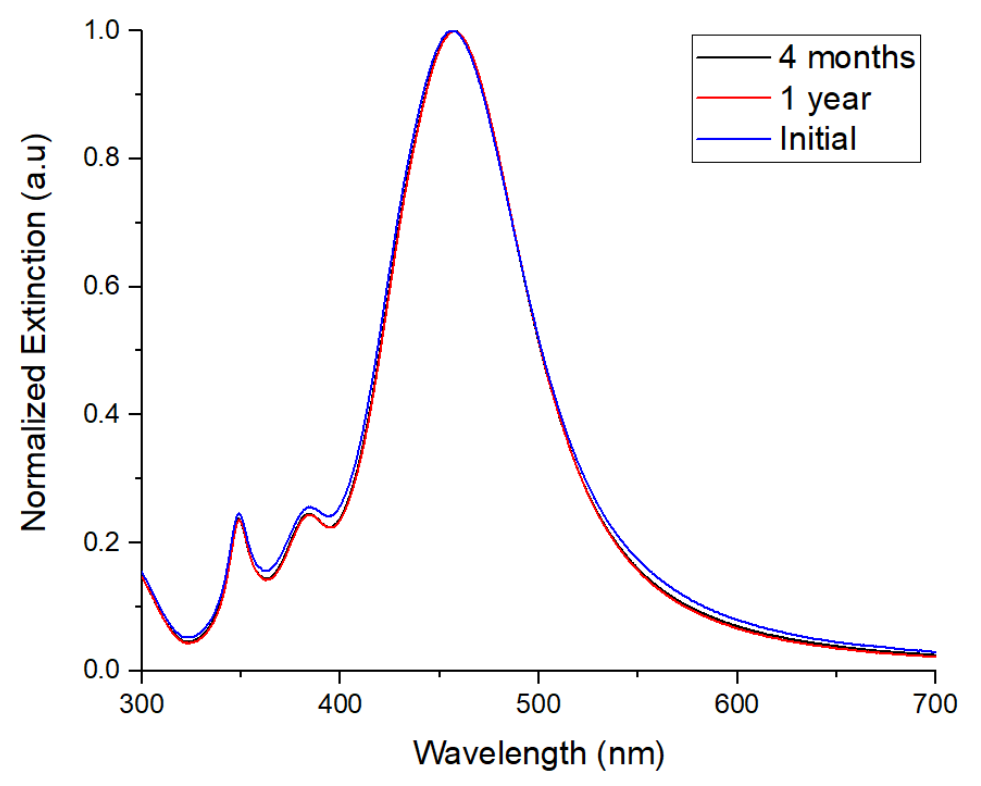

Figure 3.3: Extinction Spectra of initial AgNC solution immediately after synthesis and after 4 months and 1 year of being stored.

An additional round of TEM imaging was also conducted 4 months after the initial synthesis to evaluate if the shape and size of the AgNCs had held up over time. Sample TEM micrographs from this round of imaging and a histogram of the edge length of 162 cubes measured from the TEM images can been seen in Appendix 6.1 (Figure 6.1). The mean cube size was calculated to be $47 \mathrm{~nm} \pm 10 \mathrm{~nm}$, which is consistent with the mean cube size calculated using the initial set of TEM images. Though the average size of the cubes is consistent, there were cubes with edge lengths $>70 \mathrm{~nm}$ after 4 months that were not present in the TEMs taken after the initial synthesis. Figures $6.1 \mathrm{~B}$ and $\mathrm{C}$ also show small particles with significantly rounded edges. In nanocubes the highest energy atoms are those located at the vertices and along the 
edges, thus an observed increase in the amount of rounder small nanocubes could be an indication of Ostwald ripening, especially considering the increase in the count of larger cubes. ${ }^{169-171}$ That being said, because the mean cube sizes from Figure 3.2D and Figure 6.1D are consistent and the extinction spectra of the AgNCs remained consistent over time, it can be concluded that the AgNC solution prepared was stable over the period in which these experiments were conducted.

\subsubsection{Preparation of Polystyrene Thin Film}

Glass slides were spin-coated with a $3 \%$ weight solution of PS. The resulting slides were characterized by AFM to determine the roughness and thickness of the PS thin films. A sample AFM image of a PS thin film can be seen in Figure 3.4A below. The film was scratched (Figure 3.4B) so that the thickness of the PS thin film could be measured (shown in Figure 6.2).
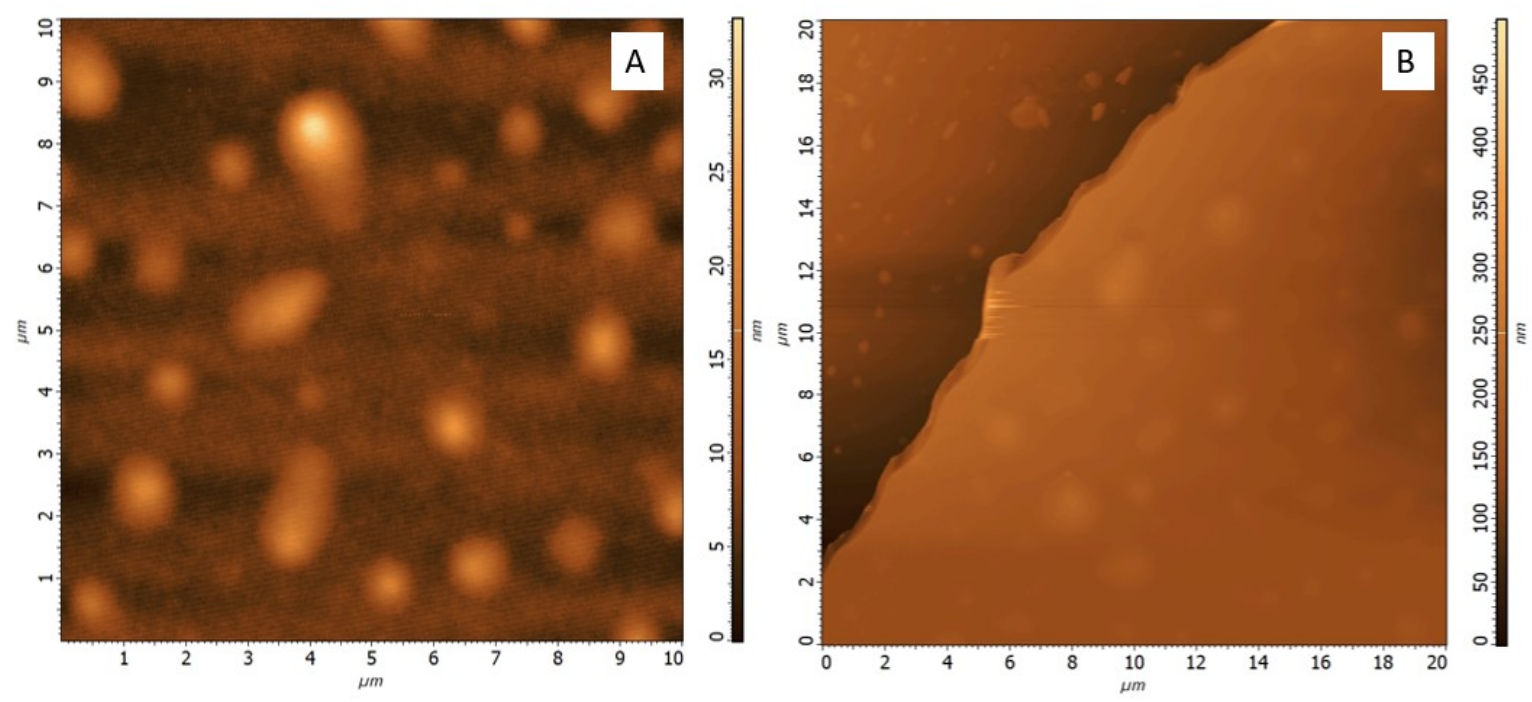

Figure 3.4: AFM images of A) a PS thin film prepared with a 3\% weight solution and B) a scratched PS thin film used for thickness measurements.

The uniformity of the AgNC monolayer deposited on the PS partially depends on the roughness of the thin film. The PS films had a roughness of $\sim 0.5 \mathrm{~nm}$ and a thickness of approximately $140 \mathrm{~nm}$. These 
values were consistent with roughness and thickness values of PS films synthesized with the spin coating method and thus deemed adequate supports for the AgNC monolayers. ${ }^{37,172}$

\subsubsection{Deposition of AgNC on to PS Thin Films via Langmuir Shaefer Deposition}

LS Deposition was used to deposit a monolayer of AgNCs on each of the PS coated glass substrates.

Figure 3.5 is a $10 \mu \mathrm{m} \times 10 \mu \mathrm{m}$ AFM image of an AgNC monolayer (right) and its corresponding extinction spectrum (left). As can be seen in the figure, a highly ordered single monolayer composed of similarly sized cubes was achieved. In the top left corner of the AFM in Figure 3.5 there is a small region in which there is no monolayer. That is likely a result of DOPC molecules that did not bind to the AgNC. While the DOPC is used as a "spacer" molecule to regulate the interparticle distance in the AgNC monolayer, excess hydrophobic DOPC molecules can interact with the hydrophobic tails of PS, adhering to the PS thin film and preventing the AgNCs from adhering to portions of the PS coated slide. Fortunately, because the hole is small, it did not interfere with the optical properties of the monolayer sample. The extinction spectra of the cubes showed a multipolar mode at $342 \mathrm{~nm}$, a quadrupolar mode at $388 \mathrm{~nm}$ and a dipolar mode at $430 \mathrm{~nm}$, indicating that the cubes retained their geometric integrity during the deposition. 

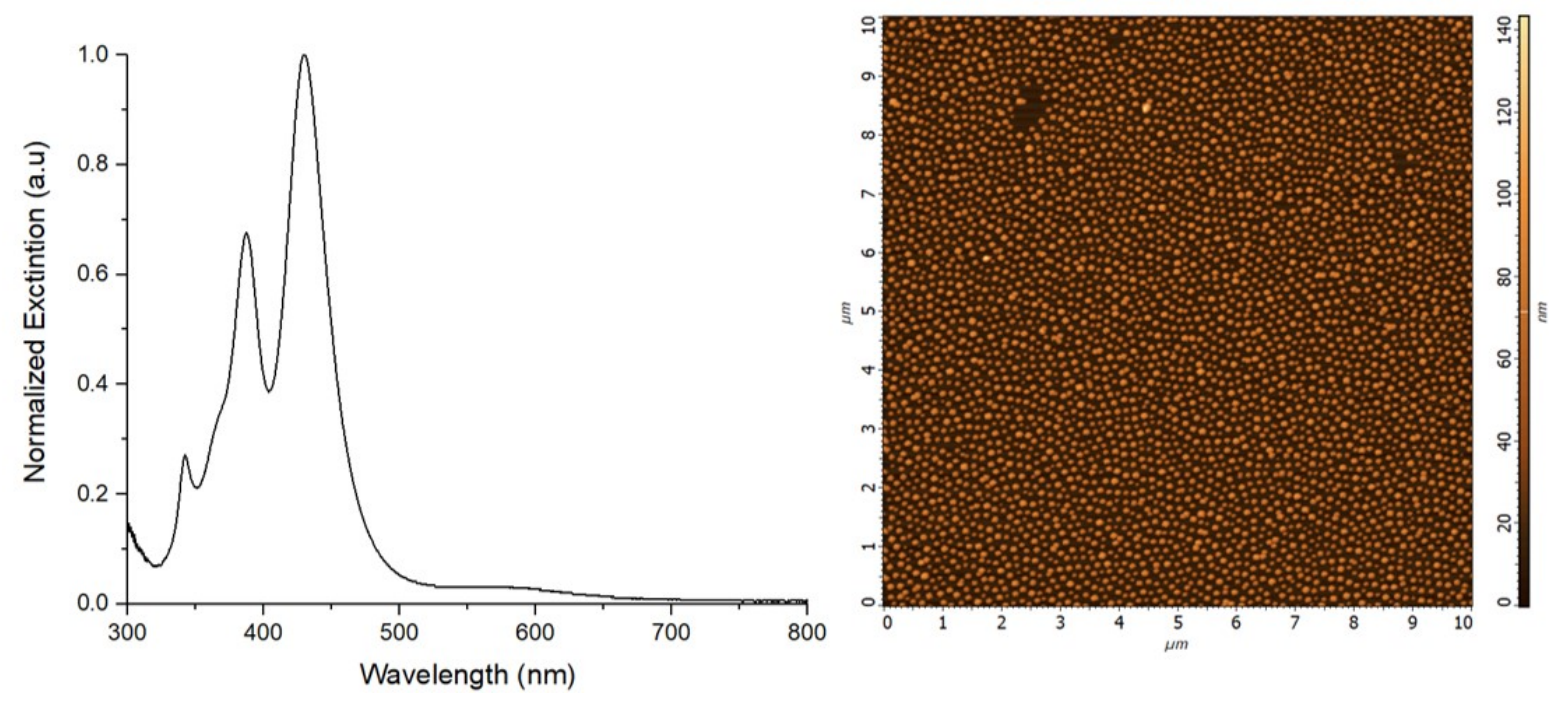

Figure 3.5: Left: Extinction spectrum of AgNC deposited on PS thin film. Right: $10 \mu \mathrm{m} \times 10 \mu \mathrm{m}$ AFM image of the same deposited AgNC monolayer.

Some of the AgNC monolayers synthesized had silver nanorods incorporated into the monolayer in addition to the AgNCs (Appendix 6.1, Figure 6.3). Ag nanorods are formed as a result of the growth of multiply twinned seed crystals in the presence of PVP. ${ }^{94}$ The presence of nanorods in the monolayers indicates that not all of the multiply twinned seed crystals were etched by the $\mathrm{NaCl}$ during synthesis. However, because these nanorods were not detected in the TEM images of the AgNC solution, not abundant in any of the monolayers, and did not affect the optical properties of the monolayers, they did not affect the quality of the produced samples.

Based on the principles of LS deposition, an increase in deposition pressure should lead to an increase in monolayer density. While the pressure of the monolayers measured by the trough during LS are arbitrary (they vary based on the density of the monolayer deposited on the trough prior to zeroing the pressure sensor) there was an observable correlation between the deposition pressure and the density of the AgNCs deposited. Appendix 6.1.3 shows AFM images of five AgNC monolayers deposited at different pressure (Figure 6.4B-E) and their corresponding extinction spectra (Figure 6.4A). As can be seen in the AFM images, the AgNC monolayers became increasingly densely packed as deposition 
pressure increased from $0 \mathrm{mN} / \mathrm{m}$ to $4 \mathrm{mN} / \mathrm{m}$. This increase in monolayer density was confirmed by a progressive increase in the intensity of the dipolar, quadrupolar and multipolar modes in the extinction spectra. These results are consistent with experimental trends observed in literature for the LS deposition of AgNC assemblies. ${ }^{49}$

An additional phenomenon observed in some AgNC monolayers deposited at high pressures was interparticle coupling. Figure 6.5 in the Appendix depicts the AFMs of monolayers deposited at $2 \mathrm{mN} / \mathrm{m}$, $4 \mathrm{mN} / \mathrm{m}, 6 \mathrm{mN} / \mathrm{m}$ and $8 \mathrm{mN} / \mathrm{m}$ along with their extinction spectra. The monolayers deposited at 2, 4 and $6 \mathrm{mN} / \mathrm{m}$ follow the trend of increased extinction spectra intensity with increased monolayer deposition density, but the monolayer deposited at $8 \mathrm{mN} / \mathrm{m}$ does not. This is because the extinction spectrum of the $8 \mathrm{mN} / \mathrm{m}$ sample has a fourth broad peak at $\sim 580 \mathrm{~nm}$ which diminishes the relative intensity of dipolar, quadrupolar and multipolar modes. Based on LSPR theory and results from previous studies of LS deposited monolayers of AgNCs, the extinction spectra suggest interparticle coupling which occurs in a monolayer due to decreased interparticle distance and/or increased cluster formation. ${ }^{39,49}$ This was confirmed by the AFMs of the monolayers in question which illustrate an increased number of particle clusters in the sample deposited at $8 \mathrm{mN} / \mathrm{m}$ compared to the monolayers deposited at lower pressures (Figure 6.5E).

\subsection{Determination of Laser conditions for Selective Thermoplasmonic} Embedment

Previous work by the lanoul lab had demonstrated that the thermoplasmonic patterning of AgNC/PS composites can result in three different states following exposure: partial embedment of the nanocubes with little to no morphological change in the monolayer; full embedment of a portion of the cubes and partial surface deformation of the polymer film; and complete ablation of the polymer film. ${ }^{149}$ Because the goal of this project was to investigate selective thermoplasmonic embedment with little to no 
damage of the underlying PS, pilot experiments needed to be conducted to determine the conditions that would result in the first state.

\subsubsection{Laser Conditions Required for Selective Thermoplasmonic Embedment of Individual Nanocubes with $458 \mathrm{~nm}$ Laser}

For thermoplasmonic heating of individual nanoparticles a laser wavelength that corresponds to the frequency of the peak in the extinction spectrum of the particles is typically used to achieve maximum

localized heating. ${ }^{55,61,74,76}$ Experimental UV-Vis spectra of the AgNCs in solution showed a strongly resolved dipolar mode at $\sim 460 \mathrm{~nm}$. Because of this, and the fact that $458 \mathrm{~nm}$ light has been shown to successfully thermoplasmonically pattern AgNC monolayers, $458 \mathrm{~nm}$ light was selected to preferentially excite individual nanocubes over their clustered counterparts. ${ }^{149}$

The $458 \mathrm{~nm}$ laser was first set at $40 \mathrm{~mW}$. Ablation (decomposition and displacement) of the film occurred at $40 \mathrm{~mW}$ after only 15 seconds, and partial deformation of the film occurred at $40 \mathrm{~mW}$ after only 5 seconds (Figure 6.6A and B). Even after the power was lowered to $23 \mathrm{~mW}$ complete embedment of the particles still occurred even at short exposure times (Figure 6.6C and D). Conversely, $13 \mathrm{~mW}$ was insufficient power for embedment to occur even after 5 minutes of exposure. The first signs of partial embedment occurred at $17 \mathrm{~mW}$ with a 5 minute exposure time (Figure 6.7). Subsequent tests revealed exposure time at that power could be reduced to 3 minute and still achieve partial embedment (Figure 6.7). As can be seen in Figure 6.7 , the trials conducted at $17 \mathrm{~mW}$ not only cause embedment with minimal damage to the PS film, but also cause selective embedment of the individual cubes over clusters. Consequently, the wavelength of $458 \mathrm{~nm}$ and a power of $17 \mathrm{~mW}$ were deemed promising parameters to use for selective embedment of individual cubes.

\subsubsection{Laser Conditions Required for Selective Thermoplasmonic Embedment of} Nanocube Clusters 
Thermoplasmonic heating of nanoparticle clusters experiments are far less common than those of individual nanoparticles or nanoparticle arrays. Experiments that do investigate thermoplasmonic heating of nanoparticle clusters often use an excitation wavelength that corresponds to the dipolar coupling peak of the cluster in question. ${ }^{72,79}$ Experimental UV-Vis spectra of AgNC monolayer samples that contained lots of nanocube clusters consistently exhibited a coupling peak at $~ 580 \mathrm{~nm}$ (Figure 6.5). This was in-keeping with the calculated extinction spectra of AgNC dimers with interparticle spacing of $\leq 4 \mathrm{~nm}$ reported by Hooshmand and El Sayed (Figure 1.8) which showed a dipolar peak between 550-600 $\mathrm{nm} .{ }^{39}$ Based on these results, a wavelength of $568 \mathrm{~nm}$ was selected for the intention of selectively thermoplasmonically embedding the AgClusters.

Previously conducted thermoplasmonic patterning experiments suggested that a laser power between 70-75 mW would be ideal for partial embedment of the AgClusters. ${ }^{149}$ However, since the power required to partially embed cubes differs depending on the optical density of the individual monolayer, the $568 \mathrm{~nm}$ laser was first set at a conservative power of $50 \mathrm{~mW}$. After there was no pattern observed on the monolayer after 7 minutes of cumulative exposure (Figure 6.8), the power was increased to 65 $\mathrm{mW}$. Partial embedment of the monolayer features without damage to the PS film was observed at 65 $\mathrm{mW}$ after exposure times of 2 minutes, 1 minute, and 20 seconds (Figure 6.9). Closer examination of the patterned area suggested that selective embedment of the AgClusters may have occurred (Figure 6.9D, E and F). Thus, it was concluded that $568 \mathrm{~nm}, 65 \mathrm{~mW}$ and exposure times between 20 seconds and 2 minutes were promising conditions for the selective embedment experiments of the AgClusters.

\subsection{Selective Embedment of Individual Silver Nanocubes at $458 \mathrm{~nm}$}

To accomplish selective embedment of individual AgNCs a monolayer containing dimers, trimers, tetramers and clusters of five or more cubes was exposed to a CW laser focused by an achromatic lens at $17 \mathrm{~mW}$ and $458 \mathrm{~nm}$ for various intervals. Topographical measurements of the monolayer were made 
before the initial laser exposure and following each exposure interval using AFM. A total of 10 images was collected: one taken prior to laser exposure and one each after 30s, 60s, 90s, 130s, 160s, 200s, 220 s, 250s, 370s and 610s (time measurements are cumulative). Figure 3.6 shows a selection of the AFMs of the AgNC monolayer taken after the different laser exposures (the full series of images can be seen in Figures 6.10-6.20).
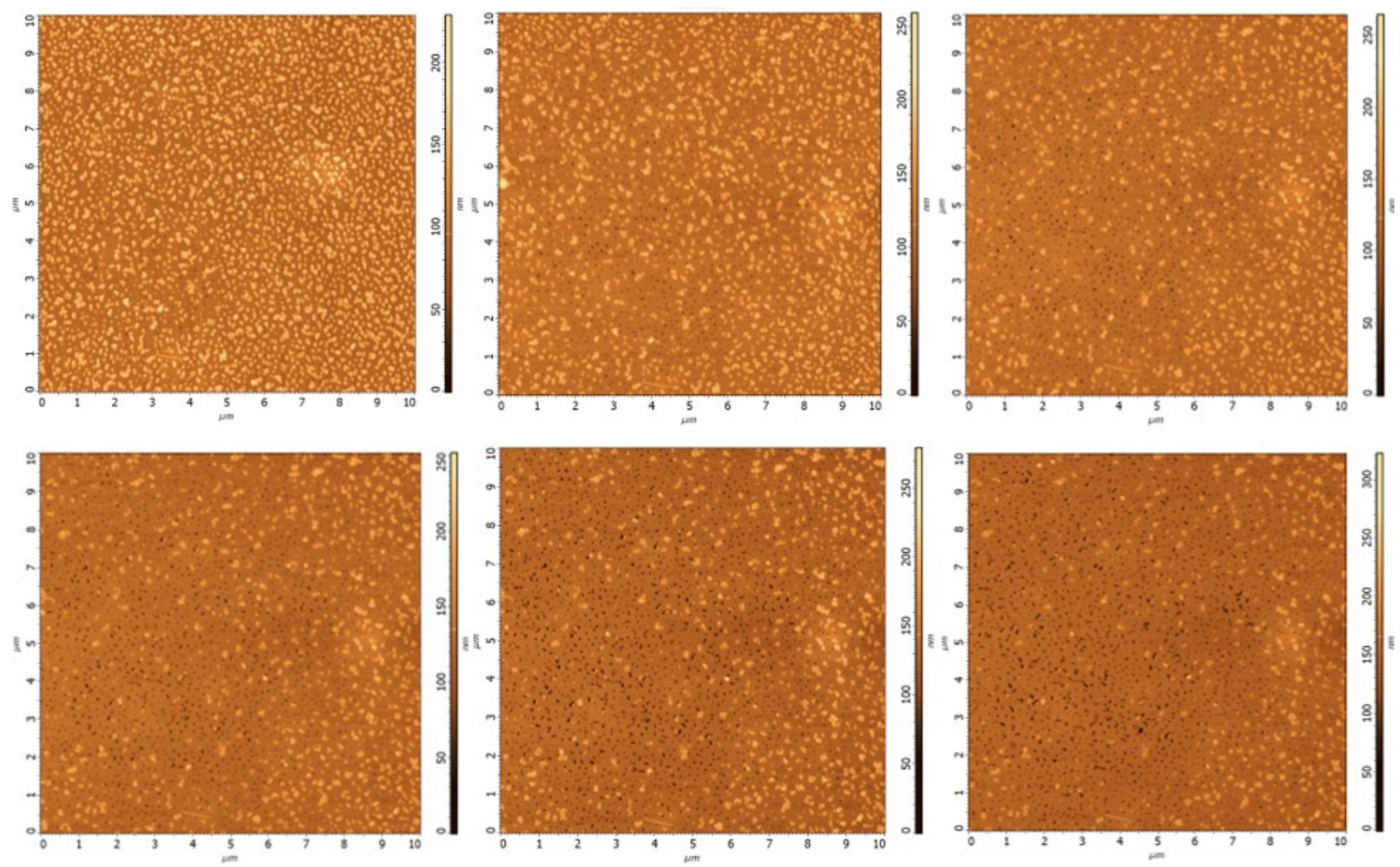

Figure 3.6: $10 \mu \mathrm{m} \times 10 \mu \mathrm{m}$ AFM images of AgNC monolayer after 30s, 90s, 160s (going left to right across the top row); 220s,

250 s and 370s (going left to right across bottom row) of exposure to $458 \mathrm{~nm}$ laser at $17 \mathrm{~mW}$. An AFM images of the surface prior to laser exposure can be seen in Figure 6.10.

The expectation of these experiments was at the shorter wavelength of $458 \mathrm{~nm}$ the individual AgNCs could selectively embed over the AgClusters, resulting in a monolayer which consisted of embedded cubes and non embedded clusters. Embedment of the various nanocrystals into the film would be 
determined by a change in surface feature topography as determined by the AFM images. Because the extent of thermoplasmonic heating of nanoparticles depends on their laser exposure time, it was also expected that the embedment depth of the individual cubes would increase with laser exposure. ${ }^{55}$

As can be seen in Figure 6.12, several small dark dots appear on the AFM monolayer following the initial 30s of laser exposure. The number of dark dots in the film increases with laser exposure (Figures 3.7 and 6.11-6.20). In the context of this type of experiment, formation of these spots in the AgNC monolayer mean one of two things: the laser has made a whole in the thin film or monolayer, or, a nanocrystal features has completely embedded in to the PS film. The size and shape of the holes formed suggest that the holes occurred as a result of individual cubes embedding. Looking at the series of images in Figures 6.11-6.20 one can observe that not only is there a progression in the number of holes formed over time, but also that the position of these holes corresponds directly to the position of individual AgNCs within the monolayer. This indicates that individual AgNCs in the monolayer embedded over the course of laser exposure.

The thermoplasmonic embedment occurred in a circular pattern (Figure 6.21) consistent to that formed by this laser system in previous experiments. Just as in the preliminary laser experiments conducted in this investigation, the quickest and most pronounced embedment occurred in the centre of the circular region (Figures 6.11-6.20). To better examine the trend in the extent and rate of embedment of the cubes at different locations, different regions of the monolayer were closely examined: one right in the centre of the circular pattern (Figure 6.22 Region I), one approximately $1.3 \mu \mathrm{m}$ out from the centre of the circular pattern (Figure 6.22 Region II) and one approximately 5.5 $\mu \mathrm{m}$ out in the top right hand corner (Figure 6.22 Region III).

In Region I two cubes appear to have completely embedded and about five are partially embedded after only the first 30 s of laser exposure (Figure 6.23A). Complete embedment of one cube and partial 
embedment of a couple of cubes is also observed in Region II after the 30s exposure (Figure 6.24A). No embedment is observed in Region III until 60s of exposure (Figure 6.25A and B). Similarly, the number of cubes that are completely and partially embedded in Regions I and II increases much more rapidly than in Region III. Of the cubes in Region III that are embedded, most of them are located in the bottom left corner of the region which is the closest to the centre of the patterned region (Figure 6.25). After the 610s of exposure essentially all of the nanocrystal features (individual cubes and AgClusters) in Regions I and II are embedded while most of the AgClusters in Region III are not. The discrepancy in embedment based on location of the cubes in the monolayer can be attributed to the fact that the power is most concentrated in the centre of the laser beam, and hence in the center of the pattern made by the laser on the film. Equations 24 and 25 in Section 1.2.2 tells us that the photothermal temperature increase exhibited by an individual nanoparticle is proportional to the power absorbed by that particle $(P)$, which is equal to the product of the irradiance $(I)$ and the absorption cross section $\left(\sigma_{a b s}\right)$. For these experiments, the irradiance experienced by the AgNCs in the monolayer is a function of how far away the particles are from the centre of the focused beam of the laser. ${ }^{149}$ Comparing Regions I, II and III it appears that at $458 \mathrm{~nm}$ and $17 \mathrm{~mW}$ a distance of $1.3 \mu \mathrm{m}$ out from the centre is not significant enough to dramatically affect the embedment rate but a distance of $5.5 \mu \mathrm{m}$ is.

Before becoming completely embedded (indicated by dark spots in the AFMs), many of the cubes appear to go through one or more partial embedment phases (indicated by the presence of darker orange spots in the AFMs). The gradual embedment process can be attributed to the fact that only a certain $\Delta T$ is achieved after each interval of illumination. ${ }^{55,61}$ After an initial interval of laser exposure the AgNCs may generate sufficient heat to temporarily heat their surrounding PS to $T_{>} T_{g}$ but not generate sufficient heat to completely melt through the PS and completely embed in the film. With each subsequent laser exposure, the AgNCs will heat again and sink a bit further. The growth of the dark holes in the film after 370 s and 610s suggest that even after completely embedding in the PS the AgNCs 
continue to heat, causing them to continue to sink deeper in the film. The occurrence of a partial embedment phase and the continual embedment of the AgNCs even past their full embedment into the PS confirm our hypothesis that the extent of embedment is correlated to the laser exposure time.

Although the embedment process is gradual, it does not occur at an equivalent rate for all cubes. In each of the three regions investigated, even in places in the film where the cubes are equally close to the centre of the laser beam (Regions I and II), some AgNCs begin to embed before others. This suggests that the $\Delta T$ and onset of heating exhibited by the cubes is not consistent. One explanation for this is that the cubes are not all identical size and geometries, and thus will have slightly different absorption cross sections and light to heat conversion efficiencies. ${ }^{72,76,79}$

What distinguished these results from previous thermoplasmonic patterning of AgNC/PS composites is the selective embedment acheived. ${ }^{149}$ Despite the significant embedment of individual AgNCs after the four initial intervals of laser exposure, small AgClusters (dimers) only begin to embed after 90s of exposure (Figure 6.13) and larger AgClusters ( $\geq 3$ cubes) only begin to embed after 220s (Figure 6.18) There is no observable complete embedment of small clusters until 130s (Figure 6.14) and no complete embedment of larger clusters until 250 s of laser exposure (Figure 6.19). Thermoplasmonic heating is a rapidly occurring process; if the AgClusters had been plasmonically excited by the $458 \mathrm{~nm}$ light they would have a rapid onset of embedment. Seeing as the AgClusters did not exhibit any sign of significant in the earliest intervals of laser exposure whereas the individual AgNCs did, it can be concluded that the AgClusters were not plasmonically excited by the $458 \mathrm{~nm}$ light. The fact that the AgClusters did eventually exhibit embedment can likely be attributed to collective heating effects contributed by the individual AgNCs and/or the cumulative effect of sustained laser exposure. ${ }^{74}$ Because the onset of embedment of the individual AgNCs and the AgClusters was temporally resolved, and the embedment of the individual AgNC occurred to such a significant extent, it can be concluded that these experiments were successful in achieving selective embedment of individual AgNCs. 
As discussed in section 1.1.6, the shape of a nanostructure is pivotal in determining which wavelengths of light the structure will absorb, and hence which wavelengths of light will be able to heat the structure..$^{55}$ The fact that the AgClusters did not embed to the same extent as the individual AgNCs at $458 \mathrm{~nm}$ suggests that the AgClusters have an entirely unique plasmonic profile from the individual AgNCs. If the individual AgNCs in the AgCluster were still able to absorb $458 \mathrm{~nm}$ light efficiently there would have been observable embedment of the AgClusters following one of the earliest intervals of laser exposure. Because there was not, it suggests that the AgNCs do not retain their individual plasmonic properties once in the cluster

\subsection{Selective Embedment of Silver Nanocubes Clusters at $568 \mathrm{~nm}$}

To perform selective embedment of the AgClusters a monolayer containing dimers, trimers, tetramers and clusters of 5 or more cubes was exposed to a CW laser focused by an achromatic lens at $568 \mathrm{~nm}$ and $65 \mathrm{~mW}$ for various intervals. A set of 6 AFM images was collected: one before laser exposure and one each after 30 s, 60 s, 90 s, 120 s and 180 s of exposure (time measurements are cumulative). AFM images of the monolayer before the initial laser exposure and following each exposure can be seen below in Figure 3.7. 

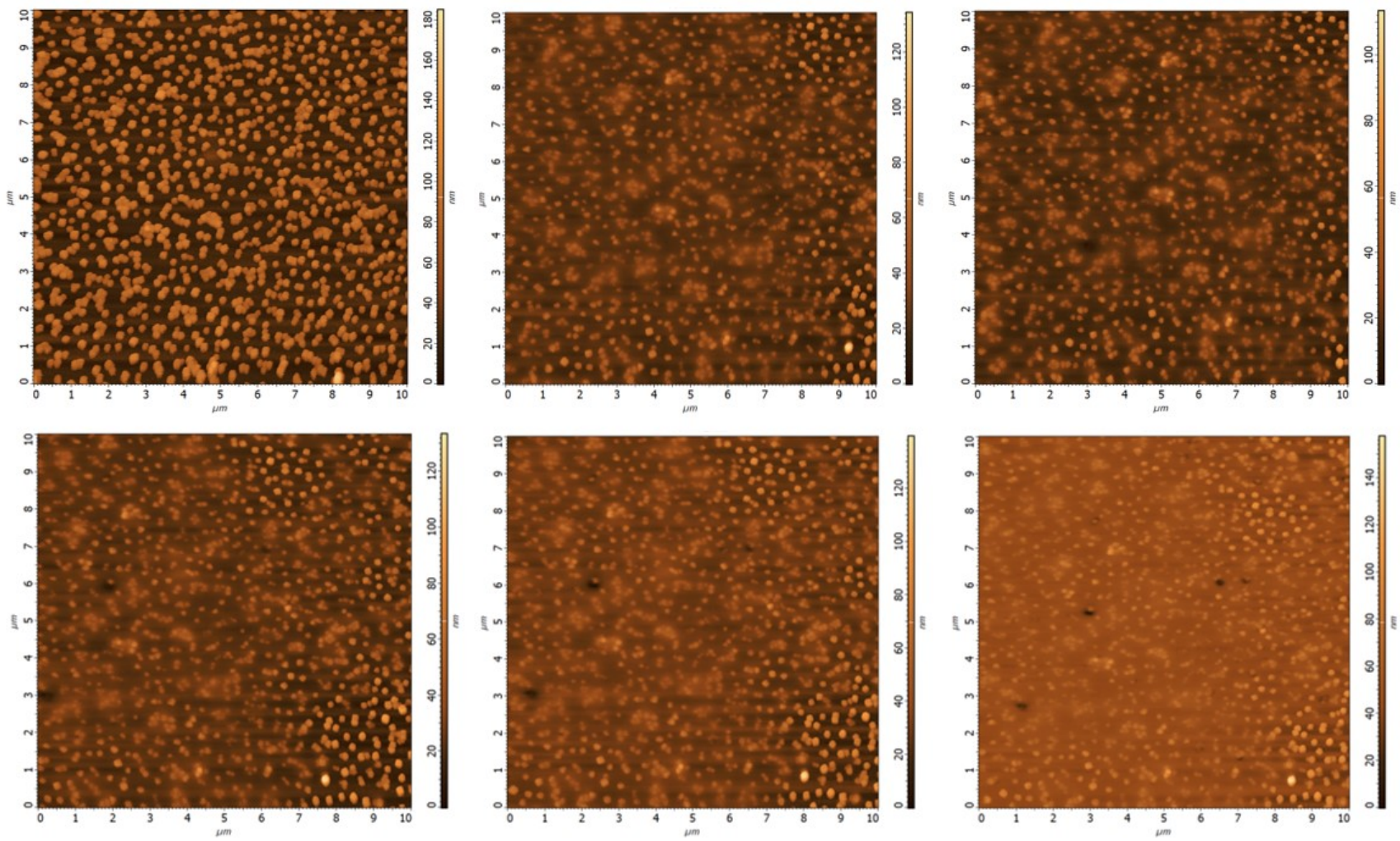

Figure 3.7: $10 \mu \mathrm{m} \times 10 \mu \mathrm{m}$ AFM images of AgNC monolayer before exposure to $568 \mathrm{~nm}$ laser at $65 \mathrm{~mW}$, after 30s, 60s (going left to right across top row), 90s, 120 s and 180 s (going left to right across bottom row).

The expectation of these experiments was that the AgClusters would embed and the individual AgNCs would not, resulting in a monolayer which consisted of embedded clusters and cubes that retained their height. It was also expected that the embedment depth of the clusters would increases with laser exposure.

Unlike in the selective embedment experiments performed at $458 \mathrm{~nm}$, the $10 \mu \mathrm{m} \times 10 \mu \mathrm{m}$ AFM images in Figure 3.7 did not yield conclusive evidence of uniform selective embedment. While there was visible embedment of the monolayer features following laser exposure, the selectivity of the embedment seemed to depend much more on how close the monolayer region was to the centre of the laser beam. In regions of the monolayer on perimeter of the patterned area (such as the top right corner of the AFMs in Figure 3.7) there was observable selective embedment of clusters. The individual cubes near 
the perimeter of the patterned area retained their topography through all of the cycles of laser exposure whereas the clusters appeared to partially embed after just the initial 30s of laser exposure (Figure 3.7). In the regions of the monolayer nearer to the centre of the patterned region, both individual AgNCs and AgClusters exhibited partial embedment, seemingly to the same degree. Even when different regions inside the patterned area were examined more closely (Figures 6.26-6.29), it was impossible to distinguish if there was any difference in the extent of embedment that had occurred for the different structures purely by the AFM images. Because it was impossible to determine purely by visual inspection of the AFM images if there was any difference in the embedment depth of the individual AgNCs and the AgClusters, more quantitative analysis need to be performed.

\subsubsection{Measurement of Height Change Selective Embedment of Cluster Experiments}

To determine if selective embedment of the AgClusters had occurred at $568 \mathrm{~nm}$, measurements of the topographical height change of different AgNCs (individual and in AgClusters) were made using the NOVA AFM software. It is important to note that this technique did not measure the embedment depth specifically but instead measured the height change $(\Delta \mathrm{h})$ over time which serves as a reasonable approximation of the embedment. The expectations were that even in the regions of the sample where both solo AgNCs and AgClusters were partially embedded by the laser, the AgClusters would have exhibited more embedment. The height change of different sizes of AgNCs (both individually and in AgClusters) was also investigated with the expectation that the different sizes of cubes would display different height changes.

Ideally, in the AFMs of the AgNC monolayers, the surface of the PS would register as 0 on the z-axis so that any measurement of the height of the different cubes would be a legitimate reflection of the cube's actual height. Unfortunately, in all of the AFMs collected there was a considerable background height signal. To combat this, various cross sections were used to measure the height signal of the PS film at 
different locations of the monolayer (Figures 6.30-6.32). These values were then averaged to obtain an average background height signal for each image (Tables 6.1-6.3), which was subtracted from the raw height measurements that were taken. This process made the heights of the AgNC features measured prior to embedment more accurate and helped correct for discrepancies in the scale between the different images.

To compare the difference in height change $(\Delta h)$ exhibited by individual AgNCs and the cubes in AgClusters following laser excitation, the average height changes for the cubes before and after 30s of laser exposure $\left(\Delta \mathrm{h}_{0-30 \mathrm{~s}}\right)$ and before and after the total $180 \mathrm{~s}$ of laser exposure $\left(\Delta \mathrm{h}_{0-180 \mathrm{~s}}\right)$ were calculated. The motivation for comparing $\Delta \mathrm{h}_{0-30 \mathrm{~s}}$ was that most of the embedment of the features in the monolayer appeared to have occurred after the initial 30s of laser exposure. An additional motivation for measuring $\Delta \mathrm{h}_{0-30 \mathrm{~s}}$ was that it is the most reflective measurement of the photothermal $\Delta \mathrm{T}$ of the different particles and clusters in air. The motivation for measuring $\Delta \mathrm{h}_{0-180 \mathrm{~s}}$ was that comparing it with $\Delta \mathrm{h}_{0-30 \mathrm{~s}}$ provided insight into the height change exhibited by particles once they are partially embedded in polystyrene and helped determine whether the hypothesis about increased embedment with increased laser exposure was correct.

The three different regions of the patterned area that were initially selected for closer analysis of embedment (Figure 6.26 Regions A, B and C) were selected as the areas in which the height measurements of the cubes would be made. These regions were selected on the basis that they were in the patterned area of interest, they contained a variety of AgClusters and individual cubes of various sizes, and they were present in all of the AFM images taken after the laser exposures (the exact $10 \mu \mathrm{m}$ $10 \mu \mathrm{m}$ area imaged in each of the AFMs varied due to frame shift). The exact cubes measured in Regions A, B and C and their classification (size and type of AgCluster they are in) can be seen in Figures 6.336.35. The height of all of the cubes outlined in Figures 6.33-6.35 was measured before laser exposure and after each progressive laser exposure for a total of six height measurements per cube. The full list of 
these measurements can be seen in Tables 6.4-6.9. For sake of brevity, only the values of $\Delta \mathrm{h}_{0-30 \mathrm{~s}}$ and $\Delta \mathrm{h}_{0 \text { - }}$ 180s are reported in this section in Tables 3.1-3.4 below. The standard deviation on some of the $\Delta \mathrm{h}$ values reported in Tables 3.1-3.4 is rather large; however, that can be attributed to the number of cubes whose heights were measured and the significant variance in the height change exhibited by the cubes whose heights were measured (even those in the same cluster). The purpose of these experiments was to compare the general embedment behaviour of individual AgNCs to the general embedment behaviour of cubes in AgClusters; hence, it was the most logical to compare the average $\Delta \mathrm{h}$ of the two different types of particles, instead of looking at the height change on a per-cube basis. While this approach does result in comparing values with large standard deviation, inspection of the individual height measurements in Tables 6.4-6.9 confirm that conclusions draw from the values in Tables 3.1-3.4 are substantiated by the overall trends in the raw data.

Table 3.1: Average Change in Height After Initial 30s Laser Exposure ( $\left.\Delta h_{0-30 s}\right)$ and after Total 3 min of Exposure ( $\left.\Delta h_{0-180 s}\right)$ for Cubes in Region $A$

\begin{tabular}{|c|c|c|}
\hline Type of Cube & $\Delta \mathrm{h}_{0-30 \mathrm{~s}}(\mathrm{~nm})$ & $\Delta \mathrm{h}_{0-180 \mathrm{~s}}(\mathrm{~nm})$ \\
\hline Individual & $25.0 \pm 5.1$ & $24.8 \pm 4.8$ \\
\hline In Cluster & $35.0 \pm 3.2$ & $42.0 \pm 4.3$ \\
\hline
\end{tabular}

Table 3.2: Average Change in Height After Initial 30s Laser Exposure ( $\left.\Delta h_{0-30 s}\right)$ and after Total 3 min of Exposure $\left(\Delta h_{0-180 s}\right)$ for Cubes in Region $B$

\begin{tabular}{|c|c|c|}
\hline Type of Cube & $\Delta \mathrm{h}_{0-30 \mathrm{~s}}(\mathrm{~nm})$ & $\Delta \mathrm{h}_{0-180 \mathrm{~s}}(\mathrm{~nm})$ \\
\hline Individual & $39.3 \pm 3.3$ & $43.7 \pm 4.2$ \\
\hline In Cluster & $45.9 \pm 6.1$ & $49.5 \pm 5.7$ \\
\hline
\end{tabular}

Table 3.3: Average Change in Height After Initial 30s Laser Exposure $\left(\Delta h_{0-30 s}\right)$ and after Total 3 min of Exposure ( $\left.\Delta h_{0-180 s}\right)$ for Different Sized Cubes in Region $C$

\begin{tabular}{|c|c|c|c|}
\hline Size & Type of Cube & $\Delta \mathrm{h}_{0-30 \mathrm{~s}}(\mathrm{~nm})$ & $\Delta \mathrm{h}_{0-180 \mathrm{~s}}(\mathrm{~nm})$ \\
\hline \multirow{2}{*}{ Small } & Individual & $33.8 \pm 2.6$ & $33.3 \pm 1.7$ \\
\cline { 2 - 4 } & In Dimer & $37.4 \pm 2.6$ & $36.4 \pm 3.0$ \\
\hline \multirow{2}{*}{ Medium } & Individual & $39.0 \pm 4.6$ & $39.0 \pm 3.8$ \\
\cline { 2 - 4 } & In Dimer/Trimer & $42.2 \pm 6.7$ & $41.1 \pm 6.3$ \\
\hline
\end{tabular}




\begin{tabular}{|c|c|c|c|}
\hline \multirow{2}{*}{ Large } & Individual & $36.2 \pm 4.5$ & $38.6 \pm 6.0$ \\
\cline { 2 - 4 } & In Trimer & $41.6 \pm 10.0$ & $44.6 \pm 9.50$ \\
\hline
\end{tabular}

Table 3.4: Average Change in Height After Initial 30s Laser Exposure ( $\left.\Delta h_{0-30 s}\right)$ and after Total $3 m i n$ of Exposure ( $\Delta \boldsymbol{h}_{0-180 \mathrm{~s}}$ ) of Cubes Large Cluster in Region $\mathrm{C}$

\begin{tabular}{|c|c|c|}
\hline Which Cluster & $\Delta \mathrm{h}_{0-30 \mathrm{~s}}(\mathrm{~nm})$ & $\Delta \mathrm{h}_{0-180 \mathrm{~s}}(\mathrm{~nm})$ \\
\hline Large Cluster I & $39.9 \pm 7.9$ & $43.4 \pm 9.2$ \\
\hline Large Cluster II & $43.2 \pm 6.1$ & $45.1 \pm 6.3$ \\
\hline
\end{tabular}

In all of the regions of the monolayer investigated the cubes in the AgClusters exhibited greater $\Delta \mathrm{h}_{0-30 \mathrm{~s}}$ and $\Delta \mathrm{h}_{0-180 \mathrm{~s}}$ than the individual AgNCs. In Region $\mathrm{A}$ the cubes in AgClusters showed an average $\Delta \mathrm{h}_{0-30 \mathrm{~s}}$ that was $10 \mathrm{~nm}$ greater than their solo AgNCs counterparts and an average $\Delta \mathrm{h}_{0-180 \text { s }}$ that was over $17 \mathrm{~nm}$ more than the $\Delta \mathrm{h}_{0-3 \min }$ measured for the individual cubes. While the difference between the $\Delta \mathrm{h}_{0-30 \text { s }}$ and $\Delta \mathrm{h}_{0-180 \mathrm{~s}}$ of the cubes in AgClusters and the cubes not in clusters was not as significant in Region $\mathrm{B}$, the cubes in AgClusters still exhibited a $6.6 \mathrm{~nm}$ greater $\Delta \mathrm{h}_{0-30 \mathrm{~s}}$ and a $5.8 \mathrm{~nm}$ greater $\Delta \mathrm{h}_{0-180 \mathrm{~s}}$ than their solo counterparts. The reason why both types of cubes in Region A exhibited greater embedment than in Region B is likely due to the fact that Region A is more in the centre of the patterned area and hence would experience greater irradiance from the $568 \mathrm{~nm}$ light leading to increased photothermal heating. Regardless of the discrepancy in the magnitude of height change in the two regions, both average height changes measured in the first two region support the hypotheses that AgClusters will be preferentially embedded at $568 \mathrm{~nm}$ and that embedment depth increases with laser exposure (i.e $\Delta \mathrm{h}_{0-180 \mathrm{~s}}>\mathrm{h}_{0-30 \mathrm{~s}}$ ).

In Region C, all sizes of cubes underwent a greater change in height following laser exposure when they were in AgClusters as suppose to stand alone features (Table 3.3). Small cubes in clusters had $\Delta \mathrm{h}_{0-30 s}$ and $\Delta \mathrm{h}_{0-180 \mathrm{~s}}$ values that were $3.6 \mathrm{~nm}$ and $3.1 \mathrm{~nm}$ greater than the respective values of $\Delta \mathrm{h}_{0-30 \mathrm{~s}}$ and $\Delta \mathrm{h}_{0-180 \mathrm{~s}}$ for individual cubes; medium sized cubes in AgClusters had $\Delta \mathrm{h}_{0-30 \mathrm{~s}}$ and $\Delta \mathrm{h}_{0-180 \mathrm{~s}}$ values that were $3.2 \mathrm{~nm}$ and $2.1 \mathrm{~nm}$ higher than the respective values of $\Delta \mathrm{h}_{0-30 \mathrm{~s}}$ and $\Delta \mathrm{h}_{0-180 \mathrm{~s}}$ for individual cubes; and large cubes in 
AgClusters had $\Delta \mathrm{h}_{0-30 \mathrm{~s}}$ and $\Delta \mathrm{h}_{0-180 \mathrm{~s}}$ values that were $5.4 \mathrm{~nm}$ and $6.0 \mathrm{~nm}$ greater than the respective values of $\Delta \mathrm{h}_{0-30 \mathrm{~s}}$ and $\Delta \mathrm{h}_{0-180 \mathrm{~s}}$ for individual cubes. While the values of $\mathrm{h}_{0-30 \mathrm{~s}}$ and $\Delta \mathrm{h}_{0-180 \mathrm{~s}}$ for the cubes in Region C confirm the hypothesis that longer laser exposure results in greater height change, they do not follow the trend predicted by theory in terms of thermoplasmonic heating increase with size.

Previous experimental studies and theoretical simulations have determined that the amount of thermoplasmonically generated heat is strongly dependent on nanoparticle size, where an increase in size should lead to increased heating. ${ }^{54,55,75}$ Based on this, the large cubes (both individual and in AgClusters) should have exhibited the greatest values of $\mathrm{h}_{0-30 \mathrm{~s}}$ and $\Delta \mathrm{h}_{0-180 \mathrm{~s}}$. In Table 3.3 one can see that it is actually the medium cubes (both individual and in AgClusters) that exhibited the greatest $\mathrm{h}_{0-30 \mathrm{~s}}$ and the individual medium cubes that exhibited the greatest $\Delta \mathrm{h}_{0-180 \mathrm{~s}}$. Some of the factors regarding why larger AgNC features may have exhibited less embedment are discussed in detail Section 3.6 but an important factor to note is that the sample size of large and medium cubes measured varied significantly. Figure 6.35 shows that a total of 9 large cubes were measured ( 3 of which were in an AgCluster) whereas a total of 22 medium cubes were measured. To more accurately compare the effects that size has on thermoplasmonic heating an equal number of cubes of each size would need to be examined.

The reason why the two larger clusters in Region C in Table 3.4 were measured independently from the other cubes measured in Region C is because they contained several cubes of each size. The purpose of measuring the height change of the two large AgClusters was to determine if even much larger clusters (five or more cubes) would be thermoplasmonically heated at $568 \mathrm{~nm}$, as that wavelength was selected on the basis that it corresponded to the extinction maximum of smaller AgNC clusters, particularly dimers. As can be seen in Table 3.4, both large AgClusters exhibited considerable height change after the initial and total laser exposure. Large cluster II had the largest $\Delta \mathrm{h}_{0-30 \mathrm{~s}}$ and the largest $\Delta \mathrm{h}_{0-180 \mathrm{~s}}$ measured in Region $C$ and large cluster I had $\Delta \mathrm{h}_{0-30 \mathrm{~s}}$ and $\Delta \mathrm{h}_{0-180 \mathrm{~s}}$ consistent with those of cubes in other 
AgClusters. Most importantly, the cubes in both large AgClusters exhibited greater height change than the individual cubes not in clusters.

Across the three regions there were seven different shapes of AgCluster examined which were composed of anywhere from two (dimers) to fourteen (large cluster I) cubes. Regardless of the shape or size of the AgClusters, all of the clusters exhibited a greater height change than the individual AgNC following exposure with the $568 \mathrm{~nm}$ laser. This suggests that the wavelength of $568 \mathrm{~nm}$ is not just well suited for the selective embedment of dimers, trimers and tetramers but extended AgClusters as well.

Although the height change measurements revealed that the AgNCs in AgClusters exhibited greater height change over the different intervals of laser exposure, it was clear that there was still significant embedment of the individual AgNCs. To elucidate the reason behind the embedment exhibited by the individual AgNCs, theoretical based calculations of the absorption cross sections of individual AgNCs and various AgClusters were made.

\subsection{Theoretical Calculations of Selective Embedment at $568 \mathrm{~nm}$}

Based on thermoplasmonic theory there are two possible explanations for the embedment exhibited by the individual AgNCs at $568 \mathrm{~nm}$. The first explanation is that both the individual AgNCs and the AgClusters absorb the $568 \mathrm{~nm}$ light but that the clusters do so more efficiently resulting in a more significant temperature increase per $\mathrm{W} / \mathrm{m}^{2}$ than the individual cubes. This means that the amount of irradiance the clusters require to reach $\Delta \mathrm{T}>\mathrm{Tg}$, would be less than that of the cubes, and would explain in regions of the sample where the irradiance was lower why the clusters were able to partially embed and the individual cubes were not. It would also explain why in regions of the sample where irradiance is higher, both the AgClusters and the individual cubes were able to partially embed in the PS thin film. The second possible explanation for this pattern is that the individual AgNCs closer to the centre of the patterned were able to embed due to the collective heating affects of the monolayer. In extended arrays 
of nanoparticles, such as the AgNC monolayer used in these experiments, there is shown to be considerable collective heating across the extent of array despite the heat being generated from discrete locations. ${ }^{74,90}$ It is possible that the selective excitation of the AgClusters at high irradiance was able to produce enough heat to heat an extended region of the PS film to its $\mathrm{Tg}$, allowing the individual AgNCs to embed as well. To determine whether or not the embedment of the individual AgNCs resulted from thermoplasmonic heating of the cubes themselves or from collective heating effects, and to confirm that the selective embedment exhibited by the AgClusters was a direct result of increased thermoplasmonic heating, the absorption cross sections at $568 \mathrm{~nm}$ of various cubes and AgClusters were calculated.

\subsubsection{Calculated Absorption Cross section of Different Clusters at $568 \mathrm{~nm}$}

The coupling between incoming light and electron oscillations in a nanoparticle is the most important factor in determining said particle's thermoplasmonic efficiency. ${ }^{54-56,79}$ The thermoplasmonic efficiency of nanoparticles is most commonly measured by means of the absorption cross section $\left(\sigma_{a b s}\right)$ which can be determined both analytically and numerically. ${ }^{55}$ In this study FDTD software was used to calculate the $\sigma_{a b s}$ at $568 \mathrm{~nm}$ of various sizes of individual cubes and various sizes and shapes of AgClusters. The purpose of these calculations was two-fold. The first purpose was to confirm that the AgClusters are more efficient at converting $568 \mathrm{~nm}$ light to heat, and hence that the height change patterns observed were a result of selective embedment. The second purpose was to use the calculated $\sigma_{a b s}$ at $568 \mathrm{~nm}$ for the different sizes of individual cubes to evaluate whether the individual AgNCs exhibited embedment as a result of their own heating or other collective heating effects.

Five different clusters were modelled: dimers where the cubes were the same size (SS dimers), dimers where the cubes were different sizes (DS dimers), trimers where the cubes were in a row formation (row trimer), trimers where the cubes were in a triangle formation (triangle trimer) and a tetramer where the cubes were oriented in a diamond (diamond tetramer). These clusters were selected as the ones to 
model as they replicated clusters found in the monolayer used for the selective embedment experiments (Figures 6.27-6.29). Because the TEM images of the AgNCs revealed an average cubes size of $\sim 45 \mathrm{~nm}$, with the lower end and higher end of the size distribution being $\sim 30 \mathrm{~nm}$ and $\sim 60 \mathrm{~nm}$ respectively, each of the cluster was modelled with $30 \mathrm{~nm}, 45 \mathrm{~nm}$ and $60 \mathrm{~nm}$ sized cubes. The absorption spectra of individual cubes of all three sizes were also calculated so that their $\sigma_{a b s}$ could be compared with those of the clusters.

The $\sigma_{\mathrm{abs}}$ of the different clusters was calculated using both $0^{\circ}$ and $90^{\circ}$ polarized light. Because the polarization of the laser used for the actual selective experiments was unknown, as was orientation of the AgNC clusters on the monolayer relative to the laser, it was important to calculate the $\sigma_{\mathrm{abs}}$ at both polarizations. Within our experimental system the clusters on the monolayer could have been excited by light polarized along their axis, orthogonal to their axis or somewhere in between, hence why it is important to calculate the $\sigma_{a b s}$ at both polarizations. The actual experimental cross sections of the different clusters in the monolayer are likely some combination of the two cross sections calculated at the different polarizations.

The absorption spectra of the various individual cubes and AgClusters calculated from 300-900 nm can be seen in Figures 6.36-6.42 in Appendix 6.5.1. The values of $\sigma_{a b s}$ at $568 \mathrm{~nm}$ (henceforth denoted as $\sigma_{a b s}^{568}$ ) for each of the different cubes and clusters taken from those spectra are tabulated below in Table 3.5.

Table 3.5: FDTD Calculated Absorption Cross Section of Different AgNCs and AgNC Clusters at $568 \mathrm{~nm}$ using $0^{\circ}$ and $90^{\circ}$ Polarized Light

\begin{tabular}{|c|c|c|c|}
\hline \multirow{2}{*}{ Type of Structure } & \multirow{2}{*}{ Cube Size $(\mathrm{nm})$} & \multicolumn{2}{|c|}{ Absorption Cross section at $568 \mathrm{~nm}\left(\sigma_{a b s}^{568}, \mathrm{~m}^{2}\right)$} \\
\cline { 2 - 4 } & & $0^{\circ}$ polarized & $90^{\circ}$ polarized \\
\hline \multirow{3}{*}{ Individual Cube } & 30 & \multicolumn{2}{|c|}{$1.02 \times 10^{-16}$} \\
\cline { 2 - 4 } & 45 & \multicolumn{2}{|c|}{$4.21 \times 10^{-16}$} \\
\cline { 2 - 4 } & 60 & $3.40 \times 10^{-15}$ & $1.26 \times 10^{-15}$ \\
\hline \multirow{2}{*}{ SS Dimer } & 30 & $1.79 \times 10^{-14}$ & $4.86 \times 10^{-16}$ \\
\cline { 2 - 4 } & 45 & \multicolumn{2}{|c|}{} \\
\hline
\end{tabular}




\begin{tabular}{|c|c|c|c|}
\hline & 60 & $1.01 \times 10^{-14}$ & $1.34 \times 10^{-15}$ \\
\hline \multirow{3}{*}{ DS Dimer } & 30 and 45 & $1.50 \times 10^{-14}$ & $3.35 \times 10^{-16}$ \\
\cline { 2 - 4 } & 30 and 60 & $1.76 \times 10^{-14}$ & $1.01 \times 10^{-15}$ \\
\cline { 2 - 4 } & 45 and 60 & $1.82 \times 10^{-14}$ & $1.06 \times 10^{-15}$ \\
\hline \multirow{3}{*}{ Row Trimer } & 30 & $9.14 \times 10^{-15}$ & $1.55 \times 10^{-16}$ \\
\cline { 2 - 4 } & 45 & $6.88 \times 10^{-15}$ & $5.90 \times 10^{-16}$ \\
\cline { 2 - 4 } & 60 & $4.06 \times 10^{-15}$ & $8.44 \times 10^{-15}$ \\
\hline \multirow{3}{*}{ Triangle Trimer } & 30 & $2.4 \times 10^{-15}$ & $4.73 \times 10^{-15}$ \\
\cline { 2 - 4 } & 45 & $1.68 \times 10^{-14}$ & $1.73 \times 10^{-14}$ \\
\cline { 2 - 4 } & 60 & $1.72 \times 10^{-14}$ & $1.26 \times 10^{-14}$ \\
\hline Diamond Tetramer & 30 & $2.26 \times 10^{-15}$ & $1.79 \times 10^{-14}$ \\
\cline { 2 - 4 } & 45 & $1.28 \times 10^{-14}$ & $1.15 \times 10^{-14}$ \\
\cline { 2 - 4 } & 60 & $1.96 \times 10^{-14}$ & $7.76 \times 10^{-15}$ \\
\hline
\end{tabular}

In order for our hypothesis that the AgClusters are selectively embedded at $568 \mathrm{~nm}$ to hold true from a theoretical standpoint, all of the different clusters would have to have a greater $\sigma_{a b s}^{568}$ than the individual cubes (the greater the absorption cross section the greater $\Delta \mathrm{T}$ via Equation 25 ). As can been seen in Table 3.5, the $\sigma_{a b s}^{568}$ of the individual AgNCs at $568 \mathrm{~nm}$ are significantly lower overall than the $\sigma_{a b s}^{568}$ of most the AgClusters. The exceptions are the $\sigma_{a b s}^{568}$ of the SS dimers, the DS dimers and the row trimers calculated with $90^{\circ}$ polarized light which are very close to the $\sigma_{a b s}^{568}$ of the individual cubes. However, as mentioned above, the polarizations are just used as a formality and the actual $\sigma_{a b s}^{568}$ exhibited by the clusters is likely a combination of the $\sigma_{a b s}^{568}$ calculated at the two polarizations. The $\sigma_{a b s}^{568}$ of the SS dimers, DS dimers and the row trimers calculated with $0^{\circ}$ polarization are substantially greater (anywhere from two to 163 times greater to be specific) than the $\sigma_{a b s}^{568}$ of than the individual cubes, hence it is extremely likely that these AgClusters will exhibit a greater $\Delta \mathrm{T}$ than the individual cubes at $568 \mathrm{~nm}$. Because of the fact that the triangle trimers and the diamond tetramers experience significant coupling in both the $\mathrm{x}$ and $\mathrm{y}$ direction, their calculated $\sigma_{a b s}^{568}$ are very high at both polarizations. The fact that their $\sigma_{a b s}^{568}$ are anywhere from three to 192 times the $\sigma_{a b s}^{568}$ of the individual cubes regardless of polarization indicates that they will exhibit a greater $\Delta T$ than the individual cubes at $568 \mathrm{~nm}$, regardless of the polarization of the light relative to the monolayer. Although the different AgClusters have 
different calculated $\sigma_{a b s}^{568}$, all of the AgClusters regardless of shape and size have a higher calculated $\sigma_{a b s}^{568}$ than the individual cubes, confirming the hypothesis that the AgClusters as a group will preferentially embed over the individual AgNCs at a wavelength of $568 \mathrm{~nm}$.

The results in Table 3.5 suggest that the triangle trimer and the diamond tetramer are the most efficient at converting $568 \mathrm{~nm}$ light to heat followed by the DS dimer, the SS dimer and the row trimer. Unfortunately, these trends are only somewhat reflected in the trends observed in the measured height changes of the various types of AgClusters. The experimental height change measurements made for the different types of clusters in each of the regions (Table 6.10 in Appendix 6.8) show that the diamond tetramer in Region A exhibited the greatest $\Delta \mathrm{h}_{0-180 \mathrm{~s}}$, followed by the DS dimer and the SS dimer, which exactly follows the trend predicted by the different AgCluster's $\sigma_{a b s}^{568}$. In Region B the triangle trimer has the greatest $\Delta \mathrm{h}_{0-180 \mathrm{~s}}$, the two types of dimers are tied for the second greatest $\Delta \mathrm{h}_{0-180 \mathrm{~s}}$, and the row trimer has the lowest measured $\Delta \mathrm{h}_{0-180 \text { s }}$ which again follow the results predicted by the AgCluster's $\sigma_{a b s}^{568}$. In Region C, however, the experimental results completely deviate from the trend predicted by the $\sigma_{a b s}^{568}$ (the row trimer has the most significant $\Delta \mathrm{h}_{0-180 \mathrm{~s}}$, followed by the triangle trimer, the SS dimer and the DS dimer). Despite the fact that the calculated $\sigma_{a b s}^{568}$ of the different individual cubes and AgClusters support our hypothesis that selective embedment of clusters will occur at $568 \mathrm{~nm}$ due to increased thermoplasmonic heating, they also indicate that our experimental results do not consistently follow the trends predicted by theory. Potential factors leading to this discrepancy are described in section 3.6.

Initially, based on the fact that many of the individual AgNCs in the monolayer exhibited height change after laser exposure it was speculated that the individual cubes themselves may have embedded due to having been photothermally heated by the $568 \mathrm{~nm}$ light. However, based on how small the magnitude of the calculated $\sigma_{a b s}^{568}$ of the different individual cubes is, it is unlikely that they could have reached $\mathrm{T}>\mathrm{Tg}$ based on Equation 25. This means that they must have embedded due to collective heating effects. 
Collective heating effects, along with other factors that may have led to the embedment patterns observed in the selective embedment experiments at $568 \mathrm{~nm}$, are discussed in more detail in section 3.6.

\subsubsection{Spatial Distribution of Plasmon Modes at $568 \mathrm{~nm}$}

Thermoplasmonic heating of nanoparticle clusters can result from exciting plasmons on the individual cubes in a cluster or by exciting a plasmon mode unique to the cluster itself..$^{61,173}$ The embedment patterns observed at $458 \mathrm{~nm}$ suggest that the AgClusters have a completely unique plasmonic profile from the individual AgNCs, but this distinction could change based on the wavelength of laser used. Because the whole principle of the selective embedment experiments at $568 \mathrm{~nm}$ was to avoid thermoplasmonic heating of the individual cubes, it is imperative to prove that mode excited by the 568 $\mathrm{nm}$ light is oriented over the whole cluster structure.

To determine whether or not the modes of the clusters are oriented along the whole structure or localized on one of the constituent cubes, the electric field enhancement factors (EEFs) for the five clusters modelled in section 3.5.1 were calculated at $568 \mathrm{~nm}$ using FDTD. Just as for the calculated absorption cross sections, the EEF calculations for each structure were calculated with both $0^{\circ}$ and $90^{\circ}$ polarized light.

EEFs are often calculated to determine the extent of coupling in nanoparticle clusters as it provides insight into the spatial distribution of electrons across the cluster. ${ }^{72,79}$ In nanospheres, the excitation of coupling modes (the modes unique to the cluster itself) is indicated in EEFs by the presence of a hot spot in-between particles and an enhancement around the border of each particle. ${ }^{72,79}$ In AgClusters, the excitation of a coupling mode is indicated by the presence of a hot spot in between the particles and an observable electron density at the vertexes and edges of the cubes. ${ }^{46,47}$ For the SS dimers, DS dimers and row trimers that only have extended geometry along one axis, the electron distribution across the three- 
dimensional structure can be accurately visualized from a single two-dimensional plane. For the triangle trimers and diamond tetramers more than one two-dimensional plane is required to clearly visualize the distribution of the electrons across the three-dimensional structure. The different planes used to generate the EEFs for each of the clusters can be seen in Appendix 6.5.2.

An example of the EEFs calculated at $568 \mathrm{~nm}$ for the different SS dimers can be seen in Figure below. The rest of the EEFs calculated at $568 \mathrm{~nm}$ for all of the clusters can be found in Appendix 6.5.2 as Figures 6.47-6.54. It is important to note that in some of the EEFs (such as in Figure 3.8 below) the cubes are depicted as rectangles instead of squares. This is due to the scales for the different axis generated by the Lumerical software.
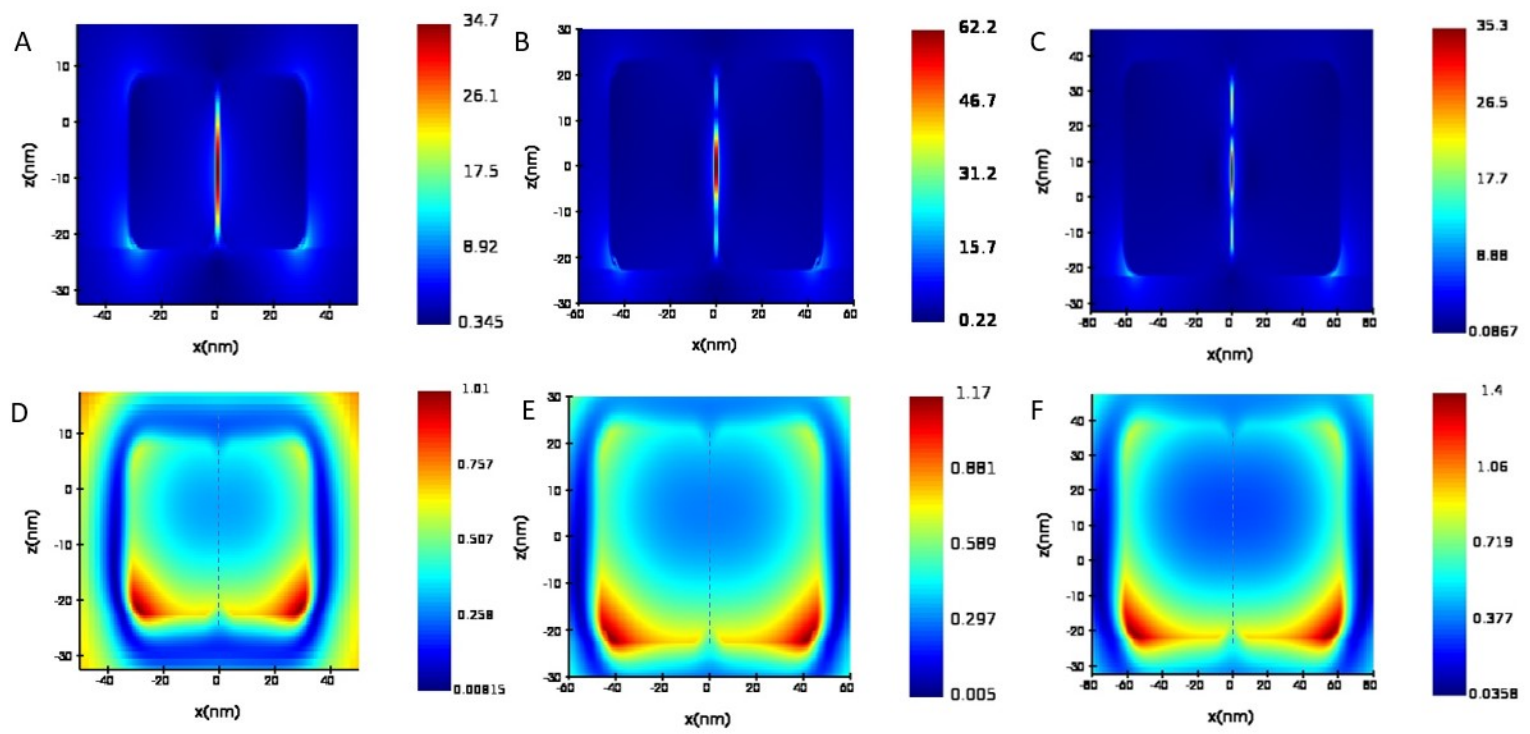

Figure 3.8: EEF of $30 \mathrm{~nm}, 45 \mathrm{~nm}$ and $60 \mathrm{~nm}$ SS dimers at $568 \mathrm{~nm}$ calculated with $0^{\circ}$ polarized light (A-C) and $90^{\circ}$ polarized light (D-F).The $2 D$ Y plane was used for the calculations with $0^{\circ}$ and $90^{\circ}$ polarized light. The coloured axis to the left of the images indicates the magnitude of the EEF. In D-F the dotted line indicates the junction between the individual cubes.

The EEFs of the SS dimers, DS dimers and row trimers calculated with $0^{\circ}$ polarized light have hot spots between the cubes and noticeable electron density at the outer corners and edges of the cubes (Figures 3.8, 6.47 and 6.48). The EEFs calculated at $90^{\circ}$ polarized light for the SS dimers and row trimers do not 
have hot spots in between the cubes but do show electron density at the outer corners and outer and bottom edges of the cubes (Figures 3.8 and 6.48). The EEFs calculated at $90^{\circ}$ polarized light for the DS dimers show a hot spot in between the two cubes in addition to the patterning at the outer corners and outer and bottom edges (Figure 6.47). The triangle trimers and diamond tetramers have EEF hot spots in nanoparticle junctions and noticeable electron density at the outer corners and edges of the cubes for the EEFs calculated at both polarizations (Figures 6.49-6.54). It is important to note that the magnitude of the EEF is not an indicator of the magnitude of thermoplasmonic heating, and thus the low EEF values calculated with $90^{\circ}$ polarized light for the SS dimers, DS dimers and row trimers are not an indication of diminished thermoplasmonic efficiency at $568 \mathrm{~nm} \cdot{ }^{55}$ As the EEF images show that the plasmons excited on each cluster by $568 \mathrm{~nm}$ light are distributed over the whole clusters, they support the hypothesis that selective thermoplasmonic excitation of all of the AgClusters occurs at $568 \mathrm{~nm}$.

\subsection{Factors that Potentially Affected the Selective Embedment of Clusters at 568 $\mathrm{nm}$}

Based on the difference in the magnitude of the calculated $\sigma_{a b s}^{568}$ of the individual AgNCs and the AgClusters, it is surprising that there was not more distinguishable selective embedment. Aside from Region A where the discrepancy between the $\Delta \mathrm{h}_{0 \mathrm{~s}-30 \mathrm{~s}}$ and the $\Delta \mathrm{h}_{0 \mathrm{~s}-180 \mathrm{~s}}$ of the individual AgNCs and the AgClusters was significant, the height change exhibited by the AgClusters was only 2.10-6.6 nm greater than the height change exhibited for the individual AgNCs in Regions B and C. To determine why the two types of structures both embedded to a similar extent, factors that were likely to have increased the embedment depth of the individual AgNC and limit the embedment of the AgClusters must be considered.

Due to the long-range temperature diffusion profile of thermoplasmonic nanoparticles, large arrays of nanoparticles exhibit substantial collective heating effects. ${ }^{55}$ These collective heating effects can result in a temperature increase that is orders of magnitude above the $\Delta T$ predicted by Equation 24 and in a 
completely uniform temperature distribution across the irradiated area. ${ }^{54}$ As can be seen in Equation 26, for nanoparticle arrays where the particles are approximately the same size the collective heating is dependent on $p$, the interparticle distance and $D$, the size of the two dimensional area under illumination. ${ }^{54}$ The smaller the value of $p$ and the greater the value of $D$, the more important the collective photothermal heating effects are estimated to be. Collective heating has been reported to be significant in nanoparticle arrays with interparticle distances of several hundred nanometers, thus, it is extremely likely that these effects occurred in the monolayer used in these experiments where the average interparticle distance between AgClusters was also on the order of a couple of hundred of nanometers. ${ }^{54}$ Looking at Figure 3.7 the size of the patterned area appears to be approximately $10 \mu \mathrm{m}$. The fact that the value of $D$ for the selective embedment experiments at $568 \mathrm{~nm}$ was on the microscale increases the extent to which collective heating likely occurred. In the context of our selective embedment experiments, a uniform temperature distribution across the irradiated area would result in a uniform embedment of all features in the patterned area, which is reflected in the AFM images in Figure 3.7.

Even though the AgClusters were selectively thermoplasmonically heated at $568 \mathrm{~nm}$ they were not able to embed completely the way the individual AgNCs could at $458 \mathrm{~nm}$. This could be due to the increased size of the AgClusters relative to the individual AgNCs.

When a polymer is heated to its $\mathrm{Tg}$, the polymer reaches a fluid like state where its chains become extremely mobile. Embedment of particles occurs when the mobile polymer chains are able to flow around the particle. ${ }^{139,141,145,150}$ Due to the increased size of the AgClusters, it is possible that embedment is limited because the PS chains are not able to flow around larger structures in the same manner than they can individual particles. The possibility that the PS chains have a more challenging time flowing around larger particles could also explain why in Region C the medium sized cubes exhibited a greater height change than the large sized cubes. 
The surface energy requirement expressed in Equation 28 states that in order for a nanoparticle to begin to embed in a polymer thin film it must satisfy the Helmholtz Free energy requirement of $\gamma_{p}>\gamma_{p s}$, where $\gamma_{p}$ is the surface free energy of the particle and $\gamma_{p s}$ is interfacial surface free energy between the particle and the polymer. ${ }^{146}$ In order for a nanoparticle to completely embed in a polymer thin film it must satisfy the additional free energy inequality of $\gamma_{p}>\gamma_{s}+\gamma_{p s}$ where $\gamma_{s}$ is the polymer's free surface energy. ${ }^{146}$ Mathematically the complete embedment condition for small silver nanospheres is usually met, and AgNCs have been experimentally shown to nearly completely embed in PS thin films; however, it is possible that the increased size of AgClusters does not allow them to meet that condition. ${ }^{139,146}$ After partially embedding in a polymer thin film, a nanoparticle is subject to two opposing forces: the long range attraction forces between the particle and the polymer which act to pull the particle deeper in to the film and the entropic forces associated with the configurations of the polymer molecules and the interfacial interactions of the particle and the polymer which act to reject the particle. ${ }^{146}$ Because the nature of the attraction forces between the polymer and the particle depend only on the composition of the particle, these attraction forces will be the same for all of the individual AgNCs and AgClusters. ${ }^{146}$ The entropic forces associated with the configuration of the polymer molecules on the other hand likely change with the size of the AgNC and AgCluster. As mentioned above, the larger the particle or cluster the more the polymer chains have to flow around it to allow for its embedment. The more the polymer chains have to displace to allow for nanoparticle embedment the greater the entropic driven repulsion of the nanoparticle by the polymer film. Similarly, the larger the area of nanoparticle that is contact with the polymer the greater the extent of interfacial repulsive interactions. This increase in repulsive forces with nanoparticle size could explain why the larger individual AgNCs and the AgClusters exhibit less embedment that would be expected solely based on their predicted thermoplasmonic heating.

\subsection{Experimental Improvements and Future Work}


Although both the selective embedment of individual AgNCs at $458 \mathrm{~nm}$ and the selective embedment of AgNC clusters were successful, there are improvements that can be made for each technique going forward.

The only undesirable result in the selective embedment experiments at $458 \mathrm{~nm}$ was the onset of embedment of AgNC clusters after 90s of laser exposure. To ensure that only the individual AgNCs are embedded in future experiments, the duration of laser exposure should not exceed 90s. As the onset of embedment of small clusters occurred between $60 \mathrm{~s}$ and $90 \mathrm{~s}$ of exposure to the $17 \mathrm{~mW}$ laser, additional experiments could be conducted to determine the exact time of exposure that leads to the embedment small AgCluster at $458 \mathrm{~nm}$. Similar trials could also be conducted to determine the exact exposure time that leads to the embedment of large AgClusters at $458 \mathrm{~nm}$, which occurs somewhere between 220250 s of laser exposure at $17 \mathrm{~mW}$. If this technique is to be applied to synthesize AgNC composites with partially embedded AgNCs, it would also be beneficial to conduct further trials at slightly lower powers and/or time intervals to avoid complete embedment of the AgNC into the PS thin film.

Though the selective embedment experiments of AgNC clusters at $568 \mathrm{~nm}$ were reasonably successful, there are several adjustments that could be made to potentially increase the success and scope of this technique. As discussed in section 3.4, the most distinguishable selective embedment occurred in regions of the monolayer where laser irradiance was the lowest (Figure 3.7, top right corner of the AFMs). This suggests that a lower power of the $568 \mathrm{~nm}$ laser might be well suited for more resolved selective embedment. Further experiments could be conducted to determine if more resolved selective embedment of AgClusters could be achieved at a slightly longer wavelength.

To test the scope of the selective embedment technique studied in these investigations, future experiments could be done to determine if varying the laser wavelength could be used for selective embedment of specific types of AgClusters. While thermoplasmonic heating of a nanostructure can 
occur over a range of wavelengths, the maximum light to heat conversion will occur at the wavelength corresponding to the peak wavelength in the structure's absorption spectrum..$^{54,55,76}$ Based on the calculated absorption spectra in Figures 6.36-6.41, the optimal wavelengths to use to selectively embed the different types of AgClusters modelled are tabulated below.

Table 3.6: FDTD Calculated Peak Absorption Wavelength for Different Types of AgNC Clusters

\begin{tabular}{|c|c|c|c|}
\hline $\begin{array}{l}\text { Type of } \\
\text { Cluster }\end{array}$ & $\begin{array}{c}\text { Cube Size } \\
\text { (s) }(\mathrm{nm})\end{array}$ & $\begin{array}{c}\text { Absorption Spectrum Peak } \\
\text { Wavelength when Light Source is } 0^{\circ} \\
\text { Polarized }(\mathrm{nm})\end{array}$ & $\begin{array}{c}\text { Absorption Spectrum Peak } \\
\text { Wavelength when Light Source is } \\
90^{\circ} \text { Polarized }(\mathrm{nm})\end{array}$ \\
\hline \multirow{3}{*}{ SS Dimer } & 30 & 520 & 444 \\
\hline & 45 & 566 & 446 \\
\hline & 60 & 674 & 398 \\
\hline \multirow{3}{*}{ DS Dimer } & 30 and 45 & 558 & 404 \\
\hline & 30 and 60 & 576 & 444 \\
\hline & 45 and 60 & 622 & 400 \\
\hline \multirow{3}{*}{$\begin{array}{l}\text { Row } \\
\text { Trimer }\end{array}$} & 30 & 600 & 418 \\
\hline & 45 & 688 & 444 \\
\hline & 60 & 660 & 372 \\
\hline \multirow{3}{*}{$\begin{array}{l}\text { Triangle } \\
\text { Trimer }\end{array}$} & 30 & 514 & 538 \\
\hline & 45 & 558 & 592 \\
\hline & 60 & 596 & 626 \\
\hline \multirow{3}{*}{$\begin{array}{l}\text { Diamond } \\
\text { Tetramer }\end{array}$} & 30 & 502 & 570 \\
\hline & 45 & 542 & 650 \\
\hline & 60 & 560 & 740 \\
\hline
\end{tabular}


The EEF images of the different clusters at their respective peak absorption wavelength (Figures 6.556.63 in Appendix 6.61) reveal that all of the wavelengths listed above correspond to plasmon modes localized over the whole of their respective cluster, confirming that these wavelengths values could indeed potentially be used for selective embedment experiments of the different types of AgClusters. What is additionally interesting about the absorption spectra calculated for the different types of AgClusters is that the values of the peak absorption cross sections calculated at the $0^{\circ}$ and $90^{\circ}$ polarization for each structure are within the same order of magnitude (Figures 6.37-6.41). Typically, the wavelengths used for experimental thermoplasmonic excitation of nanoparticle clusters are those calculated using light polarized along the dimer axis, as those are thought to lead to the considerably higher absorption cross section. ${ }^{46,47,79}$ Future experiments could not only focus on using different wavelengths to selectively embed different types of cluster, but also attempt selective embedment experiments for each of the clusters at the peak wavelengths calculated with both polarizations and compare their effectiveness.

In this experiment selective thermoplasmonic embedment was used just to preferentially embed one type of supported AgNC structure over another; however, the scope of selective thermoplasmonic embedment is not just restricted to individual AgNCs and AgClusters. Theoretically, by selecting an appropriate wavelength, selective thermoplasmonic embedment could be used to preferentially embed a desired size, type or shape of nanoparticle in a mixed array containing multiple types of nanoparticle components. This would be extremely useful in fields where nanoscale patterning is used.

With a surge in interest in secure printing technologies, microscale electronics, photovoltaics and bionanomaterials, there has been an increase in demand for various techniques which can produce nanoscale patterns in nanoparticle arrays or nanoparticle polymer composites..$^{84,174}$ Common techniques for nanoscale patterning include, electron-beam lithography, dip-pen nanolithography, and plasma nanopatterning all of which require costly instrumentation and can be very time consuming. ${ }^{150,175}$ The 
selective thermoplasmonic embedment technique reported here provides a promising alternative. When combined with specifically designed nanoparticle arrays or nanoparticle polymer composites, selective embedment could be used to pattern selected areas or domains, generate colour and even draw desired shapes or letters.

While the thermoplasmonic properties of nanoparticle arrays in their entirety have been welldocumented, little-to-no research has been focused on selective thermoplasmonic heating of individual particles within an array. The work documented here represents the first reported instance of spatially resolved thermoplasmonics in a supported nanoparticle array using microscale laser excitation. Nanoparticle arrays have been harnessed for a variety of applications across the fields of medicine, material science, clean energy, chemistry, engineering and physics and numerous investigations have been made into the applications of these arrays for a localized heating applications that can be used in biosensing, catalysis, molecular imaging and water treatment. ${ }^{53,74,90,176}$ The determination that select nanocrystals within an array can be heated with simple, relatively inexpensive instrumentation opens the doors for a myriad of new possibilities in thermoplasmonic research.

\section{Conclusion}

This work investigated the selective embedment of different supported silver nanocubes (AgNCs) using selective thermoplasmonic excitation. Monolayers of AgNCs containing individual cubes and different types of AgNC clusters were deposited on polystyrene thin films using Langmuir-Blodgett techniques and then subject to different intervals of laser exposure. It was hypothesized that individual AgNCs would embed over AgClusters when a wavelength of $458 \mathrm{~nm}$ was used and AgClusters would embed over individual AgNCs when a wavelength of $568 \mathrm{~nm}$ was used. It was also hypothesised that the embedment depth of the relevant structure would increase with laser exposure. 
For the experiments conducted at $458 \mathrm{~nm}$, selective embedment of the individual AgNC was concluded purely from visual examination of topographical images taken with an atomic force microscope before and after intervals of laser exposure. Indication that many of the cubes went through a partial embedment phase preceding complete embedment confirmed the hypothesis that embedment increased with laser exposure time.

For the experiments conducted at $568 \mathrm{~nm}$ both individual AgNCs and AgNC clusters embedded. Topographic measurements of the height change taken before and after intervals of laser exposure indicated that while both individual AgNCs and AgNC clusters embedded at $568 \mathrm{~nm}$, the clusters exhibited greater height change over the duration of laser exposure. These results, combined with the increase in height change exhibited by the cubes over time, confirmed both hypotheses.

To determine whether or not the embedment of the individual AgNCs at $568 \mathrm{~nm}$ was the result of the individual cubes undergoing thermoplasmonic heating or the collective heating effects in the monolayer, the absorption cross sections at $568 \mathrm{~nm}$ of different sizes of individual AgNCs and different sizes and shape of AgClusters were calculated. The absorption cross section values for the AgClusters were significantly greater than those of the individual cubes, confirming that increased embedment of the AgNC clusters at $568 \mathrm{~nm}$ was a result of increased thermoplasmonic heating and that the embedment of the individual AgNCs could be attributed to collective heating effects. Electric field enhancement calculations were used to show that the plasmon modes excited by the $568 \mathrm{~nm}$ laser were unique to the different clusters and not just their constituent cubes, confirming that selective embedment at $568 \mathrm{~nm}$ occurred as a result of selective excitation of the AgClusters. With some revisions the selective embedment techniques used in these studies show promise for applications in nanoparticle/polymer composite synthesis, nanostructure colour generation and secure printing technologies. The spatially resolved thermoplasmonic heating shown in these studies is the first of its kind and opens the door for future research in countless other local heating applications. 


\section{References}

(1) Schuller, J. A.; Barnard, E. S.; Cai, W.; Jun, Y. C.; White, J. S.; Brongersma, M. L. Plasmonics for Extreme Light Concentration and Manipulation. Nat. Mater. 2010, 9 (3), 193-204. https://doi.org/10.1038/nmat2630.

(2) Khlebtsov, N. G.; Dykman, L. A. Optical Properties and Biomedical Applications of Plasmonic Nanoparticles. J. Quant. Spectrosc. Radiat. Transf. 2010, 111 (1), 1-35. https://doi.org/10.1016/j.jqsrt.2009.07.012.

(3) Mayer, K. M.; Hafner, J. H. Localized Surface Plasmon Resonance Sensors. Chem. Rev. 2011, 111 (6), 3828-3857. https://doi.org/10.1021/cr100313v.

(4) Tonks, L.; Langmuir, I. Oscillations in lonized Gases. Phys. Rev. 1929, 33 (2), 195-210. https://doi.org/10.1103/PhysRev.33.195.

(5) Lu, X.; Rycenga, M.; Skrabalak, S. E.; Wiley, B.; Xia, Y. Chemical Synthesis of Novel Plasmonic Nanoparticles. Annu. Rev. Phys. Chem. 2009, 60 (1), 167-192. https://doi.org/10.1146/annurev.physchem.040808.090434.

(6) Guidez, E. B.; Aikens, C. M. Quantum Mechanical Origin of the Plasmon: From Molecular Systems to Nanoparticles. Nanoscale 2014, 6 (20), 11512-11527. https://doi.org/10.1039/c4nr02225d.

(7) Pelton, M.; Aizpurua, J.; Bryant, G. Metal-Nanoparticle Plasmonics. Laser Photonics Rev. 2008, 2 (3), 136-159. https://doi.org/10.1002/lpor.200810003.

(8) Willets, K. A.; Van Duyne, R. P. Localized Surface Plasmon Resonance Spectroscopy and Sensing. Annu. Rev. Phys. Chem. 2007, 58 (1), 267-297. https://doi.org/10.1146/annurev.physchem.58.032806.104607.

(9) Blaber, M. G.; Arnold, M. D.; Ford, M. J. A Review of the Optical Properties of Alloys and Intermetallics for Plasmonics. J. Phys. Condens. Matter 2010, 22 (14). https://doi.org/10.1088/0953-8984/22/14/143201.

(10) Albert, J.; Lepinay, S.; Caucheteur, C.; DeRosa, M. C. High Resolution Grating-Assisted Surface Plasmon Resonance Fiber Optic Aptasensor. Methods 2013, 63 (3), 239-254. https://doi.org/10.1016/j.ymeth.2013.07.007.

(11) Striebel, M.; Wrachtrup, J.; Gerhardt, I. Absorption and Extinction Cross Sections and Photon Streamlines in the Optical Near-Field. Sci. Rep. 2017, 7 (1), 1-13. https://doi.org/10.1038/s41598017-15528-w.

(12) Amirjani, A.; Firouzi, F.; Haghshenas, D. F. Predicting the Size of Silver Nanoparticles from Their Optical Properties. Plasmonics 2020. https://doi.org/10.1007/s11468-020-01121-x.

(13) Duque, J. S.; Blandón, J. S.; Riascos, H. Localized Plasmon Resonance in Metal Nanoparticles Using Mie Theory. J. Phys. Conf. Ser. 2017, 850 (1). https://doi.org/10.1088/1742-6596/850/1/012017.

(14) Acharya, R. Satellite Signal Propagation, Impairments and Mitigation. In Satellite Signal Propagation, Impairments and Mitigation; 2017; pp 1-308. https://doi.org/10.1016/C2015-006267-0.

(15) Surjikov, S. T. Mie Scattering. Thermopedia. 
(16) Wang, Z.; Zhang, Z. M.; Quan, X.; Cheng, P. A Numerical Study on Effects of Surrounding Medium, Material, and Geometry of Nanoparticles on Solar Absorption Efficiencies. Int. J. Heat Mass Transf. 2018, 116, 825-832. https://doi.org/10.1016/j.ijheatmasstransfer.2017.09.014.

(17) Jain, P. K.; Lee, K. S.; El-sayed, I. H.; El-sayed, M. A. Calculated Absorption and Scattering Properties of Gold Nanoparticles of Different Size, Shape, and Composition : Applications in Biological Imaging and Biomedicine. 2006, 7238-7248. https://doi.org/10.1021/jp057170o.

(18) Chlebus, R.; Chylek, J.; Ciprian, D.; Hlubina, P. Surface Plasmon Resonance Based Measurement of the Dielectric Function of a Thin Metal Film. Sensors (Switzerland) 2018, 18 (11), 8-19. https://doi.org/10.3390/s18113693.

(19) Rycenga, M.; Cobley, C. M.; Zeng, J.; Li, W.; Moran, C. H.; Zhang, Q.; Qin, D.; Xia, Y. Controlling the Synthesis and Assembly of Silver Nanostructures for Plasmonic Applications. Chem. Rev. 2011, 111 (6), 3669-3712. https://doi.org/10.1021/cr100275d.

(20) Blaber, M. G.; Arnold, M. D.; Harris, N.; Ford, M. J.; Cortie, M. B. Plasmon Absorption in Nanospheres: A Comparison of Sodium, Potassium, Aluminium, Silver and Gold. Phys. B Condens. Matter 2007, 394 (2), 184-187. https://doi.org/10.1016/j.physb.2006.12.011.

(21) H.J. Simon, D. E. M. and J. G. W. Second Harmonic Generation with Surface Plasmons in Alkali Metals. Opt. Commun. 1975, 13 (3), 294-298. https://doi.org/https://doi.org/10.1016/00304018(75)90103-0.

(22) Wang, F.; Shen, Y. R. General Properties of Local Plasmons in Metal Nanostructures. Phys. Rev. Lett. 2006, 97 (20), 1-4. https://doi.org/10.1103/PhysRevLett.97.206806.

(23) Lal, S.; Link, S.; Halas, N. J. Nano-Optics from Sensing to Waveguiding. Nanosci. Technol. A Collect. Rev. from Nat. Journals 2009, 213-220. https://doi.org/10.1142/9789814287005_0022.

(24) Ru, E. L.; Etchegoin, P. Principles of Surface Enhanced Raman Spectroscopy; Oxford, 2009.

(25) Fong, K. E.; Yung, L. Y. L. Localized Surface Plasmon Resonance: A Unique Property of Plasmonic Nanoparticles for Nucleic Acid Detection. Nanoscale 2013, 5 (24), 12043-12071. https://doi.org/10.1039/c3nr02257a.

(26) Miller, M. M.; Lazarides, A. A. Sensitivity of Metal Nanoparticle Plasmon Resonance Band Position to the Dielectric Environment as Observed in Scattering. J. Opt. A Pure Appl. Opt. 2006, 8 (4). https://doi.org/10.1088/1464-4258/8/4/S26.

(27) Miller, M. M.; Lazarides, A. A. Sensitivity of Metal Nanoparticle Surface Plasmon Resonance to the Dielectric Environment. J. Phys. Chem. B 2005, 109 (46), 21556-21565.

https://doi.org/10.1021/jp054227y.

(28) Lee, Y. H.; Chen, H.; Xu, Q. H.; Wang, J. Refractive Index Sensitivities of Noble Metal Nanocrystals: The Effects of Multipolar Plasmon Resonances and the Metal Type. J. Phys. Chem. C 2011, 115 (16), 7997-8004. https://doi.org/10.1021/jp202574r.

(29) Kelly, K. L.; Coronado, E.; Zhao, L. L.; Schatz, G. C. The Optical Properties of Metal Nanoparticles: The Influence of Size, Shape, and Dielectric Environment. J. Phys. Chem. B 2003, 107 (3), 668677. https://doi.org/10.1021/jp026731y.

(30) Noguez, C. Surface Plasmons on Metal Nanoparticles: The Influence of Shape and Physical 
Environment. J. Phys. Chem. C 2007, 111 (10), 3806-3819. https://doi.org/10.1021/jp066539m.

(31) Meier, M.; Wokaun, A. Enhanced Fields on Large Metal Particles: Dynamic Depolarization. Opt. Lett. 1983, 8 (11), 581. https://doi.org/10.1364/ol.8.000581.

(32) Nehl, C. L.; Hafner, J. H. Shape-Dependent Plasmon Resonances of Gold Nanoparticles. J. Mater. Chem. 2008, 18 (21), 2415-2419. https://doi.org/10.1039/b714950f.

(33) Prezgot, D. Far-Field and Near-Field Optical Properties Of Strongly Interacting Silver Nanocrystals, Carleton, 2014.

(34) Mock, J. J.; Barbic, M.; Smith, D. R.; Schultz, D. A.; Schultz, S. Shape Effects in Plasmon Resonance of Individual Colloidal Silver Nanoparticles. J. Chem. Phys. 2002, 116 (15), 6755-6759.

https://doi.org/10.1063/1.1462610.

(35) Ringe, E.; McMahon, J. M.; Sohn, K.; Cobley, C.; Xia, Y.; Huang, J.; Schatz, G. C.; Marks, L. D.; Van Duyne, R. P. Unraveling the Effects of Size, Composition, and Substrate on the Localized Surface Plasmon Resonance Frequencies of Gold and Silver Nanocubes: A Systematic Single-Particle Approach. J. Phys. Chem. C 2010, 114 (29), 12511-12516. https://doi.org/10.1021/jp104366r.

(36) Burrows, N. D.; Vartanian, A. M.; Abadeer, N. S.; Grzincic, E. M.; Jacob, L. M.; Lin, W.; Li, J.; Dennison, J. M.; Hinman, J. G.; Murphy, C. J. Anisotropic Nanoparticles and Anisotropic Surface Chemistry. J. Phys. Chem. Lett. 2016, 7 (4), 632-641.

https://doi.org/10.1021/acs.jpclett.5b02205.

(37) Gale-Mouldey, A. Plasmonic Core/Half-Shell Nanoparticles: Exploring Half-Shell Growth for Au, TiO2, SiO2, and Cu2O onto Silver Nanocubes, Carleton, 2018.

(38) Zhang, S.; Bao, K.; Halas, N. J.; Xu, H.; Nordlander, P. Substrate-Induced Fano Resonances of a Plasmonic Nanocube: A Route to Increased-Sensitivity Localized Surface Plasmon Resonance Sensors Revealed. Nano Lett. 2011, 11 (4), 1657-1663. https://doi.org/10.1021/nl200135r.

(39) Hooshmand, N.; El-sayed, M. A. Collective Multipole Oscillations Direct the Plasmonic Coupling at the Nanojunction Interfaces. 2019, 116 (39), 19299-19304.

https://doi.org/10.1073/pnas.1909416116.

(40) Jain, P. K.; El-Sayed, M. A. Plasmonic Coupling in Noble Metal Nanostructures. Chem. Phys. Lett. 2010, 487 (4-6), 153-164. https://doi.org/10.1016/j.cplett.2010.01.062.

(41) Quinten, M.; Kreibig, U.; Schönauer, D.; Genzel, L. Optical Absorption Spectra of Pairs of Small Metal Particles. Surf. Sci. 1985, 156 (PART 2), 741-750. https://doi.org/10.1016/00396028(85)90244-4.

(42) Aravind, P. K.; Nitzan, A.; Metiu, H. The Interaction between Electromagnetic Resonances and Its Role in Spectroscopic Studies of Molecules Adsorbed on Colloidal Particles or Metal Spheres. Surf. Sci. 1981, 110 (1), 189-204. https://doi.org/10.1016/0039-6028(81)90595-1.

(43) Zhao, W. B.; Park, J.; Caminade, A. M.; Jeong, S. J.; Jang, Y. H.; Kim, S. O.; Majoral, J. P.; Cho, J.; Kim, D. H. Localized Surface Plasmon Resonance Coupling in Au Nanoparticles/Phosphorus Dendrimer Multilayer Thin Films Fabricated by Layer-by-Layer Self-Assembly Method. J. Mater. Chem. 2009, 19 (14), 2006-2012. https://doi.org/10.1039/b814116a.

(44) Nordlander, P.; Oubre, C.; Prodan, E.; Li, K.; Stockman, M. I. Plasmon Hybridization in 
Nanoparticle Dimers. Nano Lett. 2004, 4 (5), 899-903. https://doi.org/10.1021/nl049681c.

(45) Halas, N. J.; Lal, S.; Chang, W.-S.; Link, S.; Nordlander, P. Plasmons in Strongly Coupled Metallic Nanostructures. Chem. Rev. 2011, 111 (6), 3913-3961. https://doi.org/10.1021/cr200061k.

(46) Hooshmand, N.; Bordley, J. A.; El-Sayed, M. A. The Sensitivity of the Distance Dependent Plasmonic Coupling between Two Nanocubes to Their Orientation: Edge-to-Edge versus Face-toFace. J. Phys. Chem. C 2016, 120 (8), 4564-4570. https://doi.org/10.1021/acs.jpcc.6b01102.

(47) Bordley, J. A.; Hooshmand, N.; El-Sayed, M. A. The Coupling between Gold or Silver Nanocubes in Their Homo-Dimers: A New Coupling Mechanism at Short Separation Distances. Nano Lett. 2015, 15 (5), 3391-3397. https://doi.org/10.1021/acs.nanolett.5b00734.

(48) Grillet, N.; Manchon, D.; Bertorelle, F.; Bonnet, C.; Broyer, M.; Cottancin, E.; Lermé, J.; Hillenkamp, M.; Pellarin, M. Plasmon Coupling in Silver Nanocube Dimers: Resonance Splitting Induced by Edge Rounding. ACS Nano 2011, 5 (12), 9450-9462.

https://doi.org/10.1021/nn2041329.

(49) Mahmoud, M. A.; El-Sayed, M. A. Comparative Study of the Assemblies and the Resulting Plasmon Fields of Langmuir-Blodgett Assembled Monolayers of Silver Nanocubes and Gold Nanocages. J. Phys. Chem. C 2008, 112 (37), 14618-14625. https://doi.org/10.1021/jp8040499.

(50) Wu, Y.; Nordlander, P. Finite-Difference Time-Domain Modeling of the Optical Properties of Nanoparticles near Dielectric Substrates. J. Phys. Chem. C 2010, 114 (16), 7302-7307. https://doi.org/10.1021/jp908980d.

(51) Sherry, L. J.; Chang, S. H.; Schatz, G. C.; Van Duyne, R. P.; Wiley, B. J.; Xia, Y. Localized Surface Plasmon Resonance Spectroscopy of Single Silver Nanocubes. Nano Lett. 2005, 5 (10), 20342038. https://doi.org/10.1021/nl0515753.

(52) Nordlander, P.; Prodan, E. Plasmon Hybridization in Nanoparticles near Metallic Surfaces. Nano Lett. 2004, 4 (11), 2209-2213. https://doi.org/10.1021/nl0486160.

(53) Baffou, G.; Quidant, R. Thermo-Plasmonics : Using Metallic Nanostructures as Nano-Sources of Heat. Laser Photonics Rev. 2013, 187 (2), 171-187. https://doi.org/10.1002/Ipor.201200003.

(54) Baffou, G.; Cichos, F.; Quidant, R. Applications and Challenges of Thermoplasmonics. Nat. Mater. 2020, 19 (9), 946-958. https://doi.org/10.1038/s41563-020-0740-6.

(55) Jauffred, L.; Samadi, A.; Klingberg, H.; Bendix, P. M.; Oddershede, L. B. Plasmonic Heating of Nanostructures. Chem. Rev. 2019, 119 (13), 8087-8130.

https://doi.org/10.1021/acs.chemrev.8b00738.

(56) Baffou, G.; Bordacchini, I.; Baldi, A.; Quidant, R. Simple Experimental Procedures to Distinguish Photothermal from Hot-Carrier Processes in Plasmonics. Light Sci. Appl. 2020, 9 (1).

https://doi.org/10.1038/s41377-020-00345-0.

(57) Yu, D.; Liu, Y.; Tian, F.; Pan, X.; Sheng, X. Accurate Thermoplasmonic Simulation of Metallic Nanoparticles. J. Quant. Spectrosc. Radiat. Transf. 2017, 187, 150-160. https://doi.org/10.1016/j.jqsrt.2016.09.007.

(58) Clavero, C. Plasmon-Induced Hot-Electron Generation at Nanoparticle/Metal-Oxide Interfaces for Photovoltaic and Photocatalytic Devices. Nat. Publ. Gr. 2014, 8 (February). 
https://doi.org/10.1038/nphoton.2013.238.

(59) Inagaki, T.; Kagami, K.; Arakawa, E. T. Photoacoustic Observation of Nonradiative Decay of Surface Plasmons in Silver. Phys. Rev. B 1981, 24 (6), 3644-3646.

https://doi.org/10.1103/PhysRevB.24.3644.

(60) Brongersma, M. L.; Halas, N. J.; Nordlander, P. Plasmon-Induced Hot Carrier Science and Technology. Nat. Publ. Gr. 2015, 10 (January). https://doi.org/10.1038/nnano.2014.311.

(61) Govorov, A. O.; Richardson, H. H. Generating Heat with Metal Nanoparticles. Nano Today 2007, 2 (1), 30-38. https://doi.org/10.1016/S1748-0132(07)70017-8.

(62) Moularas, C.; Georgiou, Y.; Adamska, K.; Deligiannakis, Y. Thermoplasmonic Heat Generation Efficiency by Nonmonodisperse Core-Shell Ag0@SiO2 Nanoparticle Ensemble. J. Phys. Chem. 2019, 123, 22499-22510. https://doi.org/10.1021/acs.jpcc.9b06532.

(63) Rao, V. G.; Aslam, U.; Linic, S. Chemical Requirement for Extracting Energetic Charge Carriers from Plasmonic Metal Nanoparticles to Perform Electron-Transfer Reactions. J. Am. Chem. Soc. 2019, 141 (1), 643-647. https://doi.org/10.1021/jacs.8b11949.

(64) Jang, Y. J.; Chung, K.; Lee, J. S.; Choi, C. H.; Lim, J. W.; Kim, D. H. Plasmonic Hot Carriers Imaging: Promise and Outlook. ACS Photonics 2018, 5 (12), 4711-4723. https://doi.org/10.1021/acsphotonics.8b01021.

(65) Hu, S.; Liu, B. J.; Feng, J. M.; Zong, C.; Lin, K. Q.; Wang, X.; Wu, D. Y.; Ren, B. Quantifying Surface Temperature of Thermoplasmonic Nanostructures. J. Am. Chem. Soc. 2018, 140 (42), 1368013686. https://doi.org/10.1021/jacs.8b06083.

(66) Besteiro, L. V.; Kong, X. T.; Wang, Z.; Hartland, G.; Govorov, A. O. Understanding Hot-Electron Generation and Plasmon Relaxation in Metal Nanocrystals: Quantum and Classical Mechanisms. ACS Photonics 2017, 4 (11), 2759-2781. https://doi.org/10.1021/acsphotonics.7b00751.

(67) Kamarudheen, R.; Castellanos, G. W.; Kamp, L. P. J.; Clercx, H. J. H.; Baldi, A. Quantifying Photothermal and Hot Charge Carrier Effects in Plasmon-Driven Nanoparticle Syntheses. ACS Nano 2018, 12 (8), 8447-8455. https://doi.org/10.1021/acsnano.8b03929.

(68) Takeyasu, N.; Yamaguchi, K.; Kagawa, R.; Kaneta, T.; Benz, F.; Fujii, M.; Baumberg, J. J. Blocking Hot Electron Emission by SiO2 Coating Plasmonic Nanostructures. J. Phys. Chem. C 2017, 121 (34), 18795-18799. https://doi.org/10.1021/acs.jpcc.7b02345.

(69) Dubi, Y.; Sivan, Y. “ Hot" Electrons in Metallic Nanostructures - Non- Thermal Carriers or Heating ? Light Sci. Appl. 2019. https://doi.org/10.1038/s41377-019-0199-x.

(70) Sivan, Y. Assistance of Metal Nanoparticles in Photocatalysis - Nothing More than a Classical Heat Source. 2019, 215-233. https://doi.org/10.1039/c8fd00147b.

(71) Dubi, Y.; Un, I. W.; Sivan, Y. Thermal Effects - an Alternative Mechanism for Plasmon-Assisted Photocatalysis. Chem. Sci. 2020, 11, 5017-5027. https://doi.org/10.1039/c9sc06480j.

(72) Baffou, G.; Quidant, R.; Garcl, F. J. Nanoscale Control of Optical Heating in Complex Plasmonic Systems. ACS Nano 2010, 4 (2), 709-716. https://doi.org/10.1021/nn901144d.

(73) Bohren, C. F. How Can a Particle Absorb More than the Light Incident on It? Am. J. Phys. 1983, 51 (4), 323-327. https://doi.org/10.1119/1.13262. 
(74) Quidant, R.; Monneret, S.; Baffou, G.; Berto, P.; Berm, E.; Polleux, R. J.; Aix, H.; Universit, M.; Marseille, C.; Park, T.; Avanc, E.; Medicine, M. Photoinduced Heating of Nanoparticle Arrays. ACS Nano 2013, 7 (8), 6478-6488. https://doi.org/10.1021/nn401924n.

(75) Chien, M. H.; Brameshuber, M.; Rossboth, B. K.; Schütz, G. J.; Schmid, S. Single-Molecule Optical Absorption Imaging by Nanomechanical Photothermal Sensing. Proc. Natl. Acad. Sci. U. S. A. 2018, 115 (44), 11150-11155. https://doi.org/10.1073/pnas.1804174115.

(76) Lalisse, A.; Tessier, G.; Plain, J.; Guillaume, B. Quantifying the E Ffi Ciency of Plasmonic Materials for Near-Field Enhancement and Photothermal Conversion. J. Phys. Chem. 2015, 119, 25518-25528. https://doi.org/10.1021/acs.jpcc.5b09294.

(77) Herzog, J. B.; Knight, M. W.; Natelson, D. Thermoplasmonics: Quantifying Plasmonic Heating in Single Nanowires. Nano Lett. 2014. https://doi.org/10.1021/nl403510u.

(78) Baffou, G.; Quidant, R.; Girard, C. Thermoplasmonics Modeling : A Green 's Function Approach. Phys. Rev. B 2010, 1-11. https://doi.org/10.1103/PhysRevB.82.165424.

(79) Rangacharya, V. P.; Wu, K.; Larsen, P. E.; Thamdrup, L. H. E.; Ilchenko, O.; Hwu, E. Te; Rindzevicius, T.; Boisen, A. Quantifying Optical Absorption of Single Plasmonic Nanoparticles and Nanoparticle Dimers Using Microstring Resonators. ACS Sensors 2020, 5 (7), 2067-2075. https://doi.org/10.1021/acssensors.0c00591.

(80) Mart, E. D.; Urbano, R. R.; Rettori, C. Thermoplasmonic Maskless Lithography on Upconverting Nanocomposites Assisted by Gold Nanostars. ACS Appl. Nanomater. 2019, 2, 6764-6771. https://doi.org/10.1021/acsanm.9b01355.

(81) Pitsillides, C. M.; Joe, E. K.; Wei, X.; Anderson, R. R.; Lin, C. P. Selective Cell Targeting with LightAbsorbing Microparticles and Nanoparticles. Biophys. J. 2003, 84 (6), 4023-4032. https://doi.org/10.1016/S0006-3495(03)75128-5.

(82) Boyer, D.; Tamarat, P.; Maali, A.; Lounis, B.; Orrit, M. Photothermal Imaging of Nanometer-Sized Metal Particles among Scatterers. Science (80-. ). 2002, 297 (5584), 1160-1163. https://doi.org/10.1126/science.1073765.

(83) Mezeme, M. E.; Brosseau, C. Engineering Nanostructures with Enhanced Thermoplasmonic Properties for Biosensing. Phys. Rev. E 2013, No. 87, 1-10.

https://doi.org/10.1103/PhysRevE.87.012722.

(84) Kang, H.; Lee, J. W.; Nam, Y. Inkjet-Printed Multiwavelength Thermoplasmonic Images for Anticounterfeiting Applications. Appl. Mater. Interfaces 2018, 10.

https://doi.org/10.1021/acsami.7b19342.

(85) Hirsch, L. R.; Stafford, R. J.; Bankson, J. A.; Sershen, S. R.; Rivera, B.; Price, R. E.; Hazle, J. D.; Halas, N. J.; West, J. L. Nanoshell-Mediated near-Infrared Thermal Therapy of Tumors under Magnetic Resonance Guidance. Proc. Natl. Acad. Sci. U. S. A. 2003, 100 (23), 13549-13554.

https://doi.org/10.1073/pnas.2232479100.

(86) Hüttmann, G.; Birngruber, R. On the Possibility of High-Precision Photothermal Microeffects and the Measurement of Fast Thermal Denaturation of Proteins. IEEE J. Sel. Top. Quantum Electron. 1999, 5 (4), 954-962. https://doi.org/10.1109/2944.796317.

(87) Li, W.; Chen, X. Gold Nanoparticles for Photoacoustic Imaging. Nanomedicine 2015, 10 (2), 299- 
320. https://doi.org/10.2217/nnm.14.169.

(88) Yang, Z.; Sun, Z.; Ren, Y.; Chen, X.; Zhang, W.; Zhu, X.; Mao, Z.; Shen, J.; Nie, S. Advances in Nanomaterials for Use in Photothermal and Photodynamic Therapeutics (Review). Mol. Med. Rep. 2019, 20 (1), 5-15. https://doi.org/10.3892/mmr.2019.10218.

(89) Zharov, V. P.; Lapotko, D. O. Photothermal Imaging of Nanoparticles and Cells. IEEE J. Sel. Top. Quantum Electron. 2005, 11 (4), 733-751. https://doi.org/10.1109/JSTQE.2005.857382.

(90) Mancini, A.; Giliberti, V.; Alabastri, A.; Calandrini, E.; De Angelis, F.; Garoli, D.; Ortolani, M. Thermoplasmonic Effect of Surface-Enhanced Infrared Absorption in Vertical Nanoantenna Arrays. J. Phys. Chem. C 2018, 122 (24), 13072-13081. https://doi.org/10.1021/acs.jpcc.8b03808.

(91) Iravani, S.; Korbekandi, H.; Mirmohammadi, S. V; Zolfaghari, B. Synthesis of Silver Nanoparticles: Chemical, Physical and Biological Methods. Res. Pharm. Sci. 2014, 9 (6), 385-406.

(92) Natsuki, J. A Review of Silver Nanoparticles: Synthesis Methods, Properties and Applications. Int. J. Mater. Sci. Appl. 2015, 4 (5), 325. https://doi.org/10.11648/j.ijmsa.20150405.17.

(93) Wang, Y.; Xia, Y. Bottom-up and Top-down Approaches to the Synthesis of Monodispersed Spherical Colloids of Low Melting-Point Metals. Nano Lett. 2004, 4 (10), 2047-2050. https://doi.org/10.1021/nl048689j.

(94) Wiley, B.; Sun, Y.; Xia, Y. Synthesis of Silver Nanostructures with Controlled Shapes and Properties. Acc. Chem. Res. 2007, 40 (10), 1067-1076. https://doi.org/10.1021/ar7000974.

(95) Sun, Y.; Xia, Y. Shape-Controlled Synthesis of Gold and Silver Nanoparticles. Science (80-. ). 2002, 298 (5601), 2176-2179. https://doi.org/10.1126/science.1077229.

(96) Chen, M.; Feng, Y. G.; Wang, X.; Li, T. C.; Zhang, J. Y.; Qian, D. J. Silver Nanoparticles Capped by Oleylamine: Formation, Growth, and Self-Organization. Langmuir 2007, 23 (10), 5296-5304. https://doi.org/10.1021/la700553d.

(97) Pillai, Z. S.; Kamat, P. V. What Factors Control the Size and Shape of Silver Nanoparticles in the Citrate Ion Reduction Method? J. Phys. Chem. B 2004, 108 (3), 945-951. https://doi.org/10.1021/jp037018r.

(98) Lee, P. C.; Meisel, D. Adsorption and Surface-Enhanced Raman of Dyes on Silver and Gold Sols The Journal of Physical Chemistry (ACS Publications). J. Phys. Chem. 1982, 60439 (50 mL), 33913395.

(99) Wan, Y.; Guo, Z.; Jiang, X.; Fang, K.; Lu, X.; Zhang, Y.; Gu, N. Quasi-Spherical Silver Nanoparticles: Aqueous Synthesis and Size Control by the Seed-Mediated Lee-Meisel Method. J. Colloid Interface Sci. 2013, 394 (1), 263-268. https://doi.org/10.1016/j.jcis.2012.12.037.

(100) Zhu, Q.; Cao, Y.; Li, D.; Fang, F.; Lu, F.; Yuan, Y. A Fast Response TLC-SERS Substrate for on-Site Detection of Hydrophilic and Hydrophobic Adulterants in Botanical Dietary Supplements. New J. Chem. 2019, 43 (35), 13873-13880. https://doi.org/10.1039/c9nj02489a.

(101) Adragna, N. C.; Alla, P. K.; Pavel-Sizmore, I. E.; Paluri, A. S. L.; Yaklic, J.; Lauf, P. K. Assessment of Silver-Nanoparticles-Induced Erythrocyte Cytotoxicity through Ion Transport Studies. Cell. Physiol. Biochem. 2019, 53 (3), 532-549. https://doi.org/10.33594/000000156.

(102) Van Hyning, D. L.; Zukoski, C. F. Formation Mechanisms and Aggregation Behavior of Borohydride 
Reduced Silver Particles. Langmuir 1998, 14 (24), 7034-7046.

https://doi.org/10.1021/la980325h.

(103) Mavani, K.; Shah, M. Synthesis of Silver Nanoparticles by Using Sodium Borohydride as a Reducing Agent. Int. J. Eng. Res. Technol. 2013, 2 (3), 1-10.

(104) Mehr, F. P.; Khanjani, M.; Vatani, P. Synthesis of Nano-Ag Particles Using Sodium Borohydride. Orient. J. Chem. 2015, 31 (3), 1831-1833. https://doi.org/10.13005/ojc/310367.

(105) Vu, X. H.; Duong, T. T. T.; Pham, T. T. H.; Trinh, D. K.; Nguyen, X. H.; Dang, V. S. Synthesis and Study of Silver Nanoparticles for Antibacterial Activity against Escherichia Coli and Staphylococcus Aureus. Adv. Nat. Sci. Nanosci. Nanotechnol. 2018, 9 (2). https://doi.org/10.1088/2043-6254/aac58f.

(106) Zielińska, A.; Skwarek, E.; Zaleska, A.; Gazda, M.; Hupka, J. Preparation of Silver Nanoparticles with Controlled Particle Size. Procedia Chemistry. 2009, pp 1560-1566.

https://doi.org/10.1016/j.proche.2009.11.004.

(107) Polte, J.; Tuaev, X.; Wuithschick, M.; Fischer, A.; Thuenemann, A. F.; Rademann, K.; Kraehnert, R.; Emmerling, F. Formation Mechanism of Colloidal Silver Nanoparticles: Analogies and Differences to the Growth of Gold Nanoparticles. ACS Nano 2012, 6 (7), 5791-5802.

https://doi.org/10.1021/nn301724z.

(108) Haque, M. N.; Kwon, S.; Cho, D. Formation and Stability Study of Silver Nano-Particles in Aqueous and Organic Medium. Korean J. Chem. Eng. 2017, 34 (7), 2072-2078. https://doi.org/10.1007/s11814-017-0096-z.

(109) Zhang, Z.; Shen, W.; Xue, J.; Liu, Y.; Liu, Y.; Yan, P.; Liu, J.; Tang, J. Recent Advances in Synthetic Methods and Applications of Silver Nanostructures. Nanoscale Res. Lett. 2018, 13. https://doi.org/10.1186/s11671-018-2450-4.

(110) Kim, D.; Jeong, S.; Moon, J. Synthesis of Silver Nanoparticles Using the Polyol Process and the Influence of Precursor Injection. Nanotechnology 2006, 17 (16), 4019-4024. https://doi.org/10.1088/0957-4484/17/16/004.

(111) Khodashenas, B.; Ghorbani, H. R. Synthesis of Silver Nanoparticles with Different Shapes. Arab. J. Chem. 2019, 12 (8), 1823-1838. https://doi.org/10.1016/j.arabjc.2014.12.014.

(112) Xia, Y.; Xiong, Y.; Lim, B.; Skrabalak, S. E. Shape-Controlled Synthesis of Metal Nanocrystals: Simple Chemistry Meets Complex Physics? Angew. Chemie - Int. Ed. 2009, 48 (1), 60-103. https://doi.org/10.1002/anie.200802248.

(113) Skrabalak, S. E.; Wiley, B. J.; Kim, M.; Formo, E. V.; Xia, Y. On the Polyol Synthesis of Silver Nanostructures: Glycolaldehyde as a Reducing Agent. Nano Lett. 2008, 8 (7), 2077-2081. https://doi.org/10.1021/nl800910d.

(114) Sun, Y. Controlled Synthesis of Colloidal Silver Nanoparticles in Organic Solutions: Empirical Rules for Nucleation Engineering. Chem. Soc. Rev. 2013, 42 (7), 2497-2511. https://doi.org/10.1039/c2cs35289c.

(115) Lamer, V. K.; Dinegar, R. H. Theory, Production and Mechanism of Formation of Monodispersed Hydrosols. J. Am. Chem. Soc. 1950, 72 (11), 4847-4854. https://doi.org/10.1021/ja01167a001. 
(116) Xiong, Y.; Chen, J.; Wiley, B.; Xia, Y.; Aloni, S.; Yin, Y. Understanding the Role of Oxidative Etching in the Polyol Synthesis of Pd Nanoparticles with Uniform Shape and Size. J. Am. Chem. Soc. 2005, 127 (20), 7332-7333. https://doi.org/10.1021/ja0513741.

(117) Wiley, B.; Herricks, T.; Sun, Y.; Xia, Y. Polyol Synthesis of Silver Nanoparticles: Use of Chloride and Oxygen to Promote the Formation of Single-Crystal, Truncated Cubes and Tetrahedrons. Nano Lett. 2004, 4 (9), 1733-1739. https://doi.org/10.1021/nl048912c.

(118) Zheng, Y.; Zeng, J.; Ruditskiy, A.; Liu, M.; Xia, Y. Oxidative Etching and Its Role in Manipulating the Nucleation and Growth of Noble-Metal Nanocrystals. Chem. Mater. 2014.

https://doi.org/10.1021/cm402023g.

(119) Wiley, B. J.; Xiong, Y. Oxidative Etching for Controlled Synthesis of Metal Nanocrystals: Atomic Addition and Subtraction. Chem. Soc. Rev. 2014, 43 (17). https://doi.org/10.1039/c4cs00136b.

(120) Wiley, B. J.; Xiong, Y.; Li, Z. Y.; Yin, Y.; Xia, Y. Right Bipyramids of Silver: A New Shape Derived from Single Twinned Seeds. Nano Lett. 2006, 6 (4), 765-768. https://doi.org/10.1021/nl060069q.

(121) Lignier, P.; Bellabarba, R.; Tooze, R. P. Scalable Strategies for the Synthesis of Well-Defined Copper Metal and Oxide Nanocrystals. Chem. Soc. Rev. 2012, 41 (5), 1708-1720. https://doi.org/10.1039/c1cs15223h.

(122) Siekkinen, A. R.; McLellan, J. M.; Chen, J.; Xia, Y. Rapid Synthesis of Small Silver Nanocubes by Mediating Polyol Reduction with a Trace Amount of Sodium Sulfide or Sodium Hydrosulfide. Chem. Phys. Lett. 2006, 432 (4-6), 491-496. https://doi.org/10.1016/j.cplett.2006.10.095.

(123) Chen, J.; Herricks, T.; Xia, Y. Polyol Synthesis of Platinum Nanostructures: Control of Morphology through the Manipulation of Reduction Kinetics. Angew. Chemie - Int. Ed. 2005, 44 (17), 25892592. https://doi.org/10.1002/anie.200462668.

(124) Al-Saidi, W. A.; Feng, H.; Fichthorn, K. A. Adsorption of Polyvinylpyrrolidone on Ag Surfaces: Insight into a Structure-Directing Agent. Nano Lett. 2012, 12 (2), 997-1001. https://doi.org/10.1021/nl2041113.

(125) Koczkur, K. M.; Mourdikoudis, S.; Polavarapu, L.; Skrabalak, S. E. Polyvinylpyrrolidone (PVP) in Nanoparticle Synthesis. Dalt. Trans. 2015, 44 (41), 17883-17905. https://doi.org/10.1039/c5dt02964c.

(126) Zeng, J.; Zheng, Y.; Rycenga, M.; Tao, J.; Li, Z. Y.; Zhang, Q.; Zhu, Y.; Xia, Y. Controlling the Shapes of Silver Nanocrystals with Different Capping Agents. J. Am. Chem. Soc. 2010, 132 (25), 85528553. https://doi.org/10.1021/ja103655f.

(127) Xia, Y.; Xiong, Y.; Lim, B.; Skarabalak, S. E. Shape-Controlled Synthesis of Metal Nanocrystals : Simple Chemistry Meets Complex Physics? 2009, 48 (1), 60-103. https://doi.org/10.1002/anie.200802248.Shape-Controlled.

(128) Mouritz, A. P. Introduction to Aerospace Materials; Woodhead Publishing, 2012.

(129) Overney, R. M.; Buenviaje, C.; Luginbühl, R.; Dinelli, F. Glass and Structural Transitions Measured at Polymer Surfaces on the Nanoscale. J. Therm. Anal. Calorim. 2000, 59, 205-225.

(130) Ellison, C. J.; Torkelson, J. M. The Distribution of Glass-Transition Temperatures in Nanoscopically Confined Glass Formers. Nat. Mater. 2003, 2 (10), 695-700. https://doi.org/10.1038/nmat980. 
(131) Tsui, O. K. C.; Russell, T. P.; Hawker, C. J. Effect of Interfacial Interactions on the Glass Transition of Polymer Thin Films. Macromolecules 2001, 34 (16), 5535-5539.

https://doi.org/10.1021/ma000028v.

(132) Ediger, M. D.; Forrest, J. A. Dynamics near Free Surfaces and the Glass Transition in Thin Polymer Films: A View to the Future. Macromolecules 2014, 47 (2), 471-478.

https://doi.org/10.1021/ma4017696.

(133) Vignaud, G.; Chebil, M. S.; Bal, J. K.; Delorme, N.; Beuvier, T.; Grohens, Y.; Gibaud, A. Densification and Depression in Glass Transition Temperature in Polystyrene Thin Films. Langmuir 2014, No. 30, 11599-11608.

(134) Bushell, M. Utilizing Hybrid Plasmon Modes to Probe Nanoparticle-Polymer Interfaces, Carleton University.

(135) Polystyrene https://www.sigmaaldrich.com/catalog/product/aldrich/182427?lang=en\&region=CA (accessed Sep 8, 2020).

(136) Ferhan, A. R.; Kim, D. H. Nanoparticle Polymer Composites on Solid Substrates for Plasmonic Sensing Applications. Nano Today 2016, 11 (4), 415-434. https://doi.org/10.1016/j.nantod.2016.07.001.

(137) Zhang, Y.; Jeong, C. K.; Wang, J.; Sun, H.; Li, F.; Zhang, G.; Chen, L. Q.; Zhang, S.; Chen, W.; Wang, Q. Flexible Energy Harvesting Polymer Composites Based on Biofibril-Templated 3-Dimensional Interconnected Piezoceramics. Nano Energy 2018, 50 (May), 35-42. https://doi.org/10.1016/j.nanoen.2018.05.025.

(138) Wang, G.; Wang, C.; Zhang, F.; Yu, X. Electrical Percolation of Nanoparticle-Polymer Composites. Comput. Mater. Sci. 2018, 150 (October 2017), 102-106. https://doi.org/10.1016/j.commatsci.2018.03.051.

(139) Bushell, M.; lanoul, A. Selective Embedment of Silver Nanocrystals into Spatially Segregated Domains in Thin Polymer Films for Controlled Fabrication of Functional Nanocomposites. RSC Adv. 2019, 9 (33), 19131-19141. https://doi.org/10.1039/c9ra02490e.

(140) Bushell, M.; Bottomley, A.; Ianoul, A. Utilization of Hybrid Plasmonic Modes to Investigate Surface Interactions between Nanocubes and Polymer Substrates. Appl. Phys. A Mater. Sci. Process. 2017, 123 (2), 1-7. https://doi.org/10.1007/s00339-016-0735-y.

(141) Bottomley, A.; Prezgot, D.; Coyle, J. P.; lanoul, A. Dynamics of Nanocubes Embedding into Polymer Films Investigated: Via Spatially Resolved Plasmon Modes. Nanoscale 2016, 8 (21), 11168-11176. https://doi.org/10.1039/c6nr02604d.

(142) Raju, T. D.; Veeralingam, S.; Badhulika, S. Polyvinylidene Fluoride/ZnSnO3 Nanocube/Co3O4 Nanoparticle Thermoplastic Composites for Ultrasound-Assisted Piezo-Catalytic Dye Degradation. ACS Appl. Nano Mater. 2020, 3 (5), 4777-4787. https://doi.org/10.1021/acsanm.0c00771.

(143) Ngo, I. L.; Prabhakar Vattikuti, S. V.; Byon, C. Effects of Thermal Contact Resistance on the Thermal Conductivity of Core-Shell Nanoparticle Polymer Composites. Int. J. Heat Mass Transf. 2016, 102, 713-722. https://doi.org/10.1016/j.ijheatmasstransfer.2016.06.064.

(144) Maurer, E.; Barcikowski, S.; Gökce, B. Process Chain for the Fabrication of Nanoparticle Polymer 
Composites by Laser Ablation Synthesis. Chem. Eng. Technol. 2017, 40 (9), 1535-1543. https://doi.org/10.1002/ceat.201600506.

(145) Bushell, M.; lanoul, A. Interfacial Diffusion of Silver Nanocrystals into Polymer Surfaces Monitored by Hybrid Plasmon Modes. J. Phys. Chem. C 2018, 122 (18), 10197-10204. https://doi.org/10.1021/acs.jpcc.8b00314.

(146) Kovacs, G. J.; Vincett, P. S. Formation and Thermodynamic Stability of a Novel Class of Useful Materials: Close-Packed Monolayers of Submicron Monodisperse Spheres Just below a Polymer Surface. J. Colloid Interface Sci. 1982, 90 (2), 335-351. https://doi.org/10.1016/00219797(82)90302-2.

(147) Brandrup, J.; Immergut, E. H.; Grulke, E. A. Polymer Handbook, 4th ed.; Wiley: New York, 1999.

(148) Chaudhury, M. K. Interfacial Interaction between Low-Energy Surfaces. Mater. Sci. Eng. R Reports 1996, 16 (3), 97-159. https://doi.org/10.1016/0927-796X(95)00185-9.

(149) Prezgot, D. Functional Properties of Hybrid Resonances in Plasmonic Nanocrystals, Carleton University, 2020.

(150) Fedoruk, M.; Lutich, A. A.; Feldmann, J. Subdiffraction-Limited Milling by an Optically Driven Single Gold Nanoparticle. ACS Nano 2011, 5 (9), 7377-7382. https://doi.org/10.1021/nn2023045.

(151) Acharya, S.; Hill, J. P.; Ariga, K. Soft Langmuir-Blodgett Technique for Hard Nanomaterials. Adv. Mater. 2009, 21 (29), 2959-2981. https://doi.org/10.1002/adma.200802648.

(152) Martin, P.; Szablewski, M. NIMA Langmuir-Blodgett Troughs Operating Manual 6th Edition; 2004.

(153) Rubinger, C. P. L.; Moreira, R. L.; Cury, L. A.; Fontes, G. N.; Neves, B. R. A.; Meneguzzi, A.; Ferreira, C. A. Langmuir-Blodgett and Langmuir-Schaefer Films of Poly(5-Amino-1-Naphthol) Conjugated Polymer. Appl. Surf. Sci. 2006, 253 (2), 543-548. https://doi.org/10.1016/j.apsusc.2005.12.096.

(154) Gray, S. K. Theory and Modeling of Plasmonic Structures. J. Phys. Chem. C 2013, 117 (5), 19831994. https://doi.org/10.1021/jp309664c.

(155) Montgomery, J. M.; Lee, T. W.; Gray, S. K. Theory and Modeling of Light Interactions with Metallic Nanostructures. J. Phys. Condens. Matter 2008, 20 (32). https://doi.org/10.1088/09538984/20/32/323201.

(156) Yee, K. S. Numerical Solution. Nat. Photonics 2014, 9 (1), 5-6. https://doi.org/10.1038/nphoton.2014.305.

(157) Gedney, S. . Introduction to the Finite-Difference Time-Domain (FDTD) Method for Electromagnetics; Morgan \& Claypool Publishers, 2011.

(158) Choroszucho, A. Analysis of the Influence of the Complex Structure of Clay Hollow Bricks on the Values of Electric Field Intensity by Using the FDTD Method. Arch. Electr. Eng. 2016, 65 (4), 745759. https://doi.org/10.1515/aee-2016-0052.

(159) Mercatelli, R.; Romano, G.; Ratto, F.; Matteini, P.; Centi, S.; Cialdai, F.; Monici, M.; Pini, R.; Fusi, F. Quantitative Measurement of Scattering and Extinction Spectra of Nanoparticles by Darkfield Microscopy. Appl. Phys. Lett. 2011, 99 (13), 1-4. https://doi.org/10.1063/1.3636439.

(160) Grand, J.; Auguié, B.; Le Ru, E. C. Combined Extinction and Absorption UV-Visible Spectroscopy as 
a Method for Revealing Shape Imperfections of Metallic Nanoparticles. Anal. Chem. 2019, 91 (22), 14639-14648. https://doi.org/10.1021/acs.analchem.9b03798.

(161) Movsesyan, A.; Baudrion, A.-L.; Adam, P.-M. Extinction Measurements of Metallic Nanoparticles Arrays as a Way to Explore the Single Nanoparticle Plasmon Resonances. Opt. Express 2018, 26 (5), 6439. https://doi.org/10.1364/oe.26.006439.

(162) Near, R.; Hayden, S.; El-Sayed, M. Extinction vs Absorption: Which Is the Indicator of Plasmonic Field Strength for Silver Nanocubes? J. Phys. Chem. C 2012, 116 (43), 23019-23026. https://doi.org/10.1021/jp309272b.

(163) Chen, H.; Sun, Z.; Ni, W.; Woo, K. C.; Lin, H. Q.; Sun, L.; Yan, C.; Wang, J. Plasmon Coupling in Clusters Composed of Two-Dimensionally Ordered Gold Nanocubes. Small 2009, 5 (18), 21112119. https://doi.org/10.1002/smll.200900256.

(164) Fan, J. A.; Bao, K.; Wu, C.; Bao, J.; Bardhan, R.; Halas, N. J.; Manoharan, V. N.; Shvets, G.; Nordlander, P.; Capasso, F. Fano-like Interference in Self-Assembled Plasmonic Quadrumer Clusters. Nano Lett. 2010, 10 (11), 4680-4685. https://doi.org/10.1021/nl1029732.

(165) Devraj, S. Fundamentals of Optics, 2nd ed.; PHI Learning Pvt. Ltd, 2015.

(166) Skrabalak, S. E.; Au, L.; Li, X.; Xia, Y. Facile Synthesis of Ag Nanocubes and Au Nanocages. Nat. Protoc. 2007, 2 (9), 2182-2190. https://doi.org/10.1038/nprot.2007.326.

(167) Gale-Mouldey, A. Plasmonic Core/Half-Shell Nanoparticles: Exploring Half-Shell Growth for Au, TiO2, SiO2, and Cu2O onto Silver Nanocubes, Carleton University, 2018.

(168) Phan, H. T.; Haes, A. J. What Does Nanoparticle Stability Mean? J. Phys. Chem. C 2019, 123 (27), 16495-16507. https://doi.org/10.1021/acs.jpcc.9b00913.

(169) Gentry, S. T.; Kendra, S. F.; Bezpalko, M. W. Ostwald Ripening in Metallic Nanoparticles: Stochastic Kinetics. J. Phys. Chem. C 2011, 115 (26), 12736-12741. https://doi.org/10.1021/jp2009786.

(170) Gommes, C. J. Ostwald Ripening of Confined Nanoparticles: Chemomechanical Coupling in Nanopores. Nanoscale 2019, 11 (15), 7386-7393. https://doi.org/10.1039/c9nr01349k.

(171) Kuo, C. L.; Hwang, K. C. Does Morphology of a Metal Nanoparticle Play a Role in Ostwald Ripening Processes? Chem. Mater. 2013, 25 (3), 365-371. https://doi.org/10.1021/cm3031279.

(172) Jorgenson, E. Anisotropic Nanomaterials: Silver-Copper Oxide Core-Partial Shell Nanoparticles, Carleton University, 2017.

(173) Xie, B.; Ma, L.; Zhao, J.; Liu, L. Dependent Absorption Property of Nanoparticle Clusters: An Investigation of the Competing Effects in the near Field. Opt. Express 2019, 27 (8), A280. https://doi.org/10.1364/oe.27.00a280.

(174) Prezgot, D.; Bottomley, A.; Coyle, J. P.; lanoul, A. Unusually Sharp Localized Surface Plasmon Resonance in Supported Silver Nanocrystals with a Thin Dielectric Coating. J. Phys. Chem. Lett. 2017, 8 (22), 5555-5558. https://doi.org/10.1021/acs.jpclett.7b02499.

(175) Rogers, J. A.; Lee, H. H. Unconventional Nanopatterning Techniques and Applications; John Wiley \& Sons, Inc., 2008. https://doi.org/10.1002/9780470405789. 
(176) Grewal, G. Thermo-Plasmonic Arrays for Solar Desalination Applications, lowa State University, 2019.

\section{Appendices}

\subsection{Supplementary Material for Section 3.1}

6.1.1 Additional Transmission Electron Microscopy Characterization of AgNCs in Solution

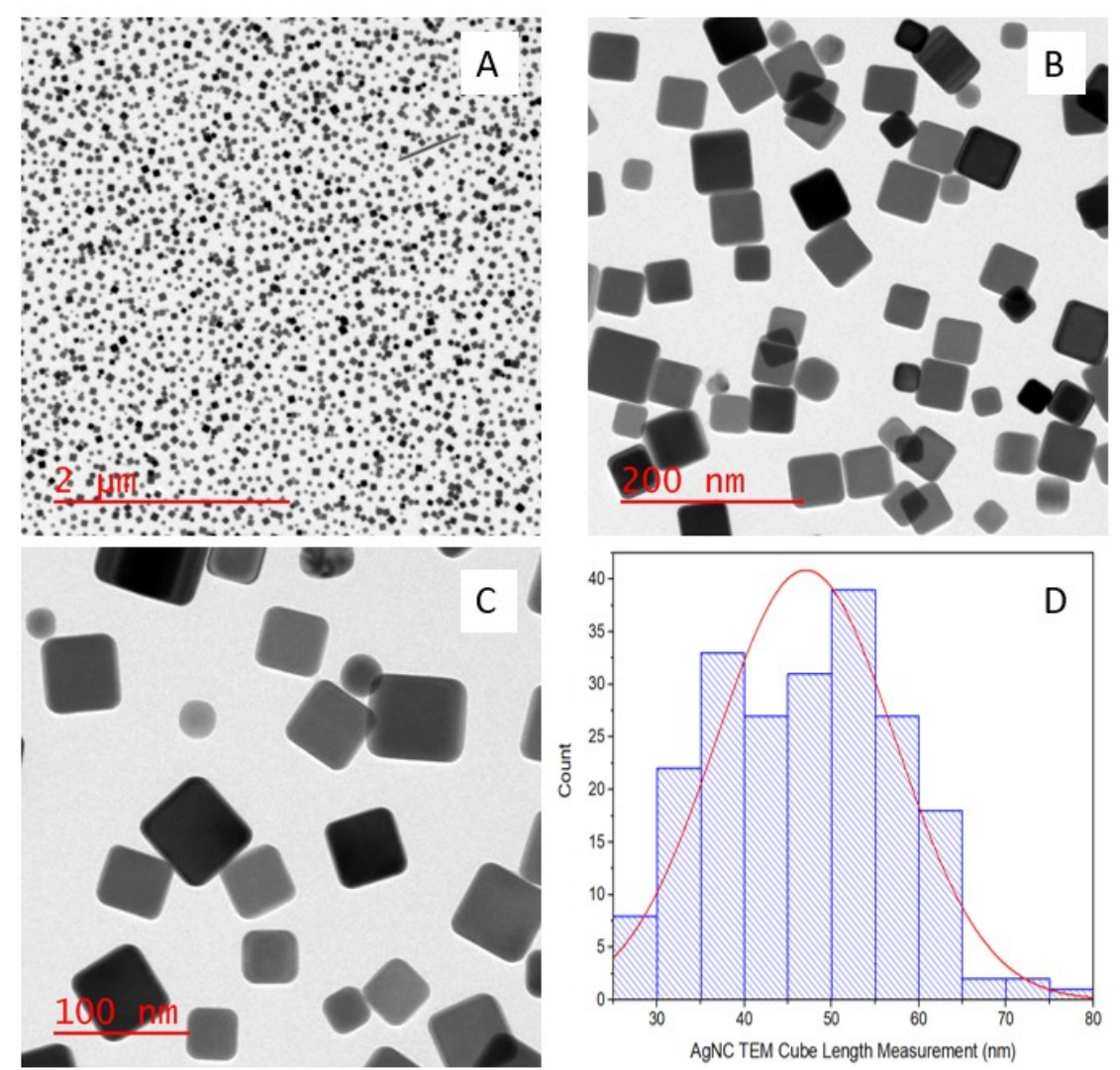

Figure 6.1: A) Large Scale TEM image of AgNC B) Medium Scale TEM image of AgNCs C) Small Scale TEM image of AgNC D) Histogram of AgNC Edge Length Measurement from TEM image set taken 4 months after initial synthesis.

6.1.2 Thickness Measurement of PS Thin Film 

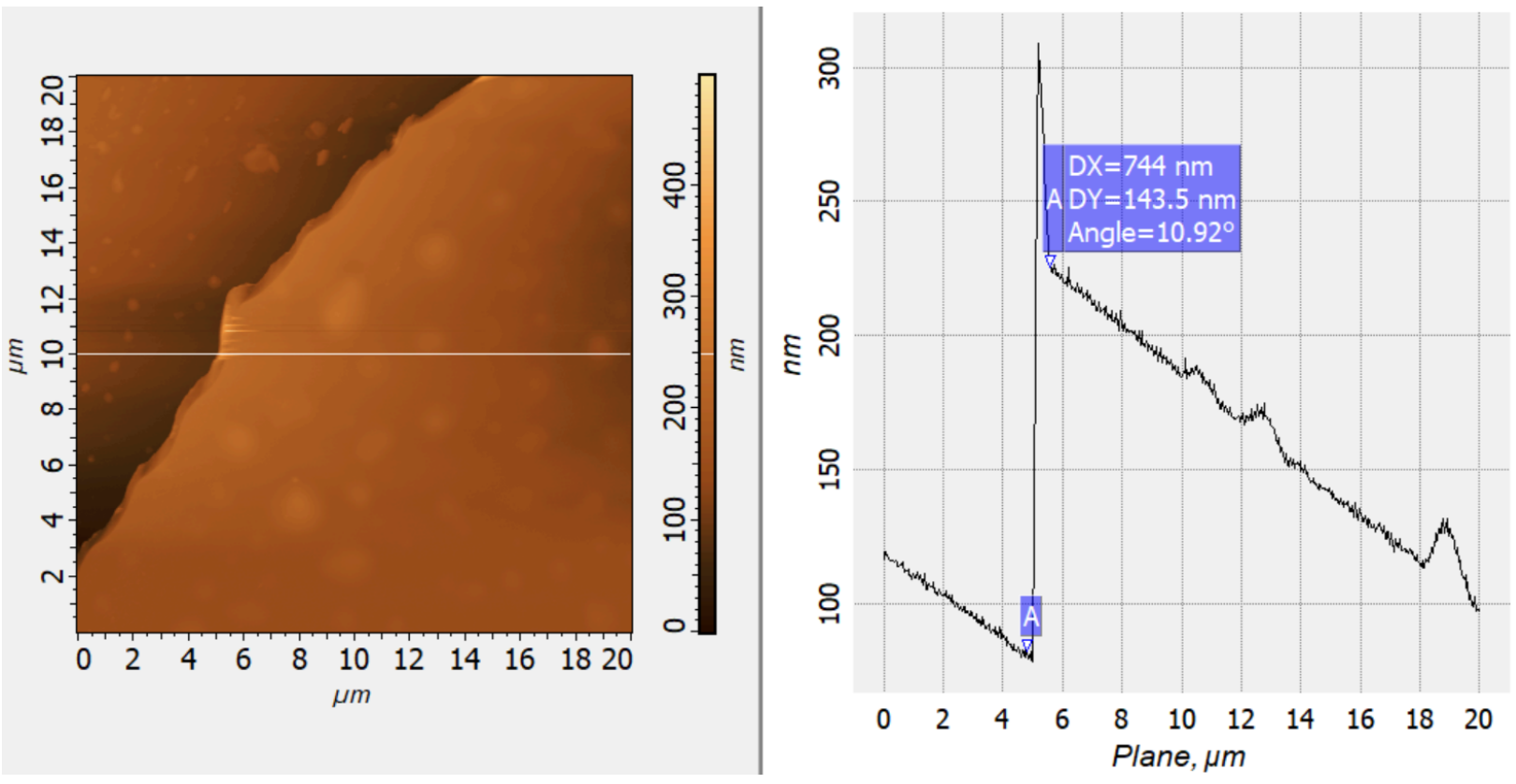

Figure 6.2: X-axis Cross section taken in Nova Software to measure thickness of PS thin Film.

6.1.3 Additional Atomic Force Microscope and UV-Vis Characterization of Supported AgNC Monolayers

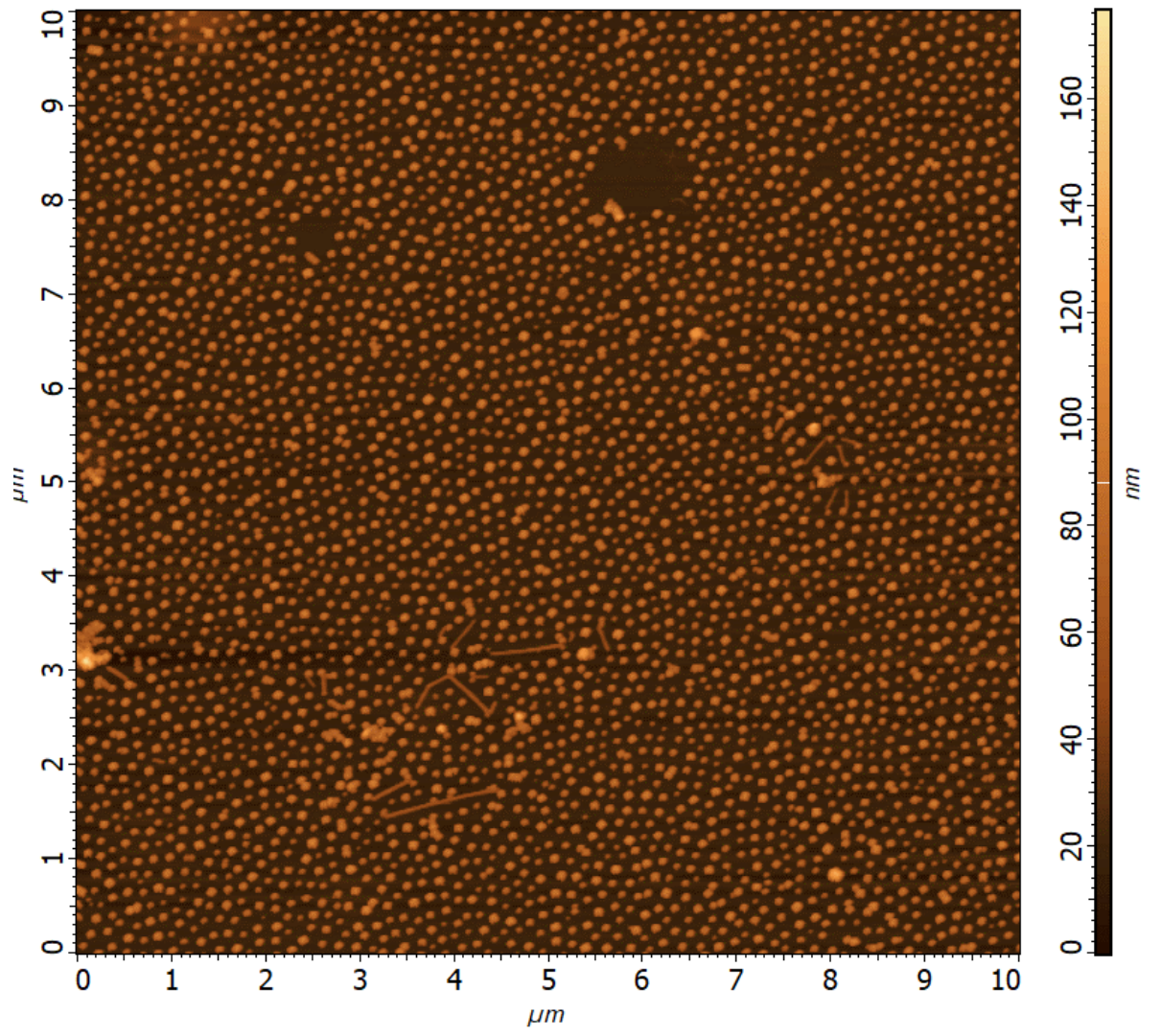

Figure 6.3: $10 \mu \mathrm{m} \times 10 \mu \mathrm{m}$ AFM of Deposited AgNC monolayer on PS with Nanorods. 

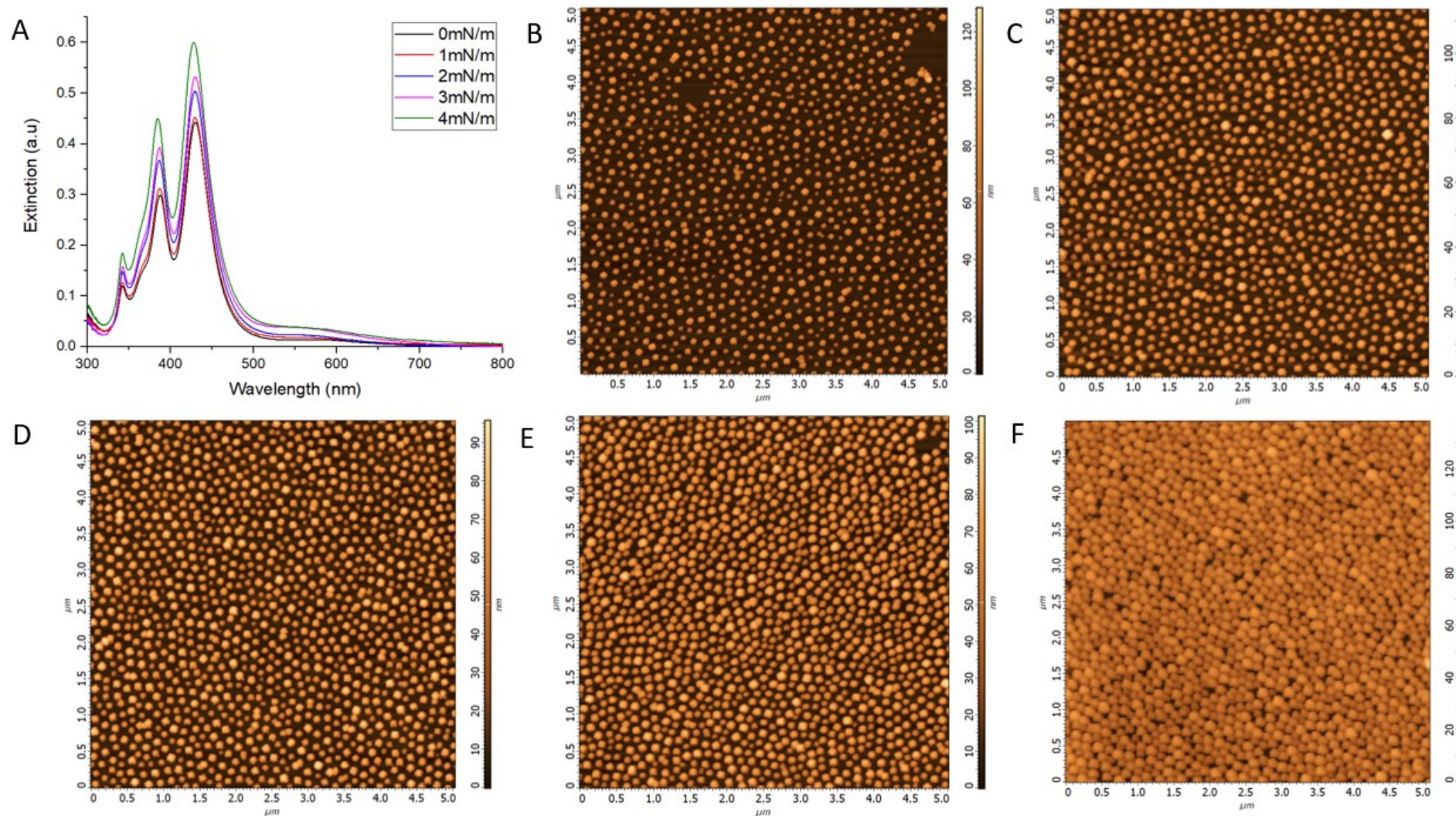

Figure 6.4: Extinction spectra (A) and $5 \mu \mathrm{m} \times 5 \mu \mathrm{m}$ AFM images of AgNC monolayers deposited at pressures of $0 \mathrm{mN} / \mathrm{m}$ (B), 1 $\mathrm{mN} / \mathrm{m}(C), 2 \mathrm{mN} / \mathrm{m}(D), 3 \mathrm{mN} / \mathrm{m}(E)$ and $4 \mathrm{mN} / \mathrm{m}(F)$. 

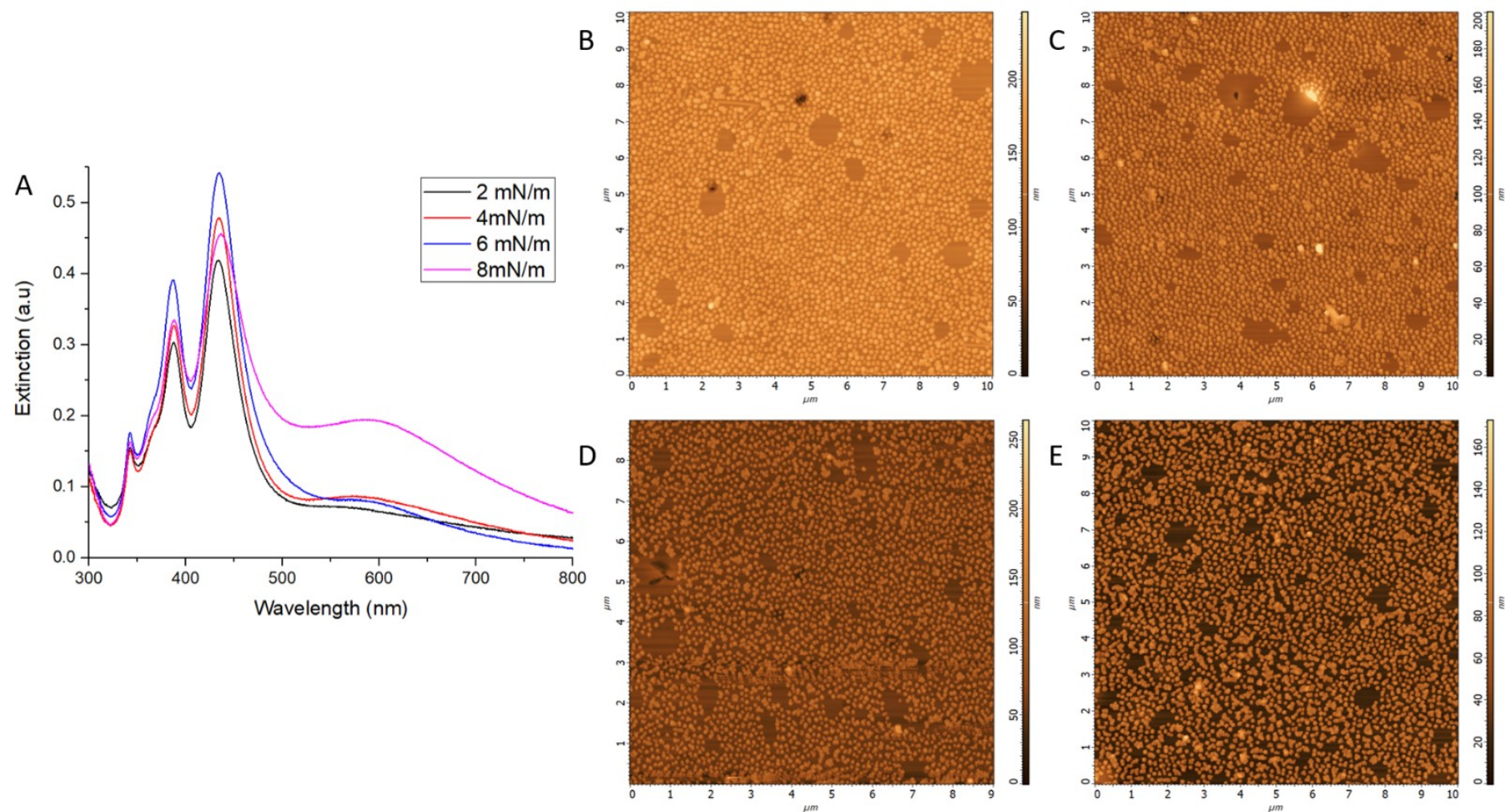

Figure 6.5: Extinction spectra (A) and $10 \mu \mathrm{m} \times 10 \mu \mathrm{m}$ AFMs of AgNC monolayers deposited at pressures of $2 \mathrm{mN} / \mathrm{m}(B), 4$ $\mathrm{mN} / \mathrm{m}(C), 6 \mathrm{mN} / \mathrm{m}(D), 8 \mathrm{mN} / \mathrm{m}$ (E). 


\subsection{Supplementary Material for Section 3.2}
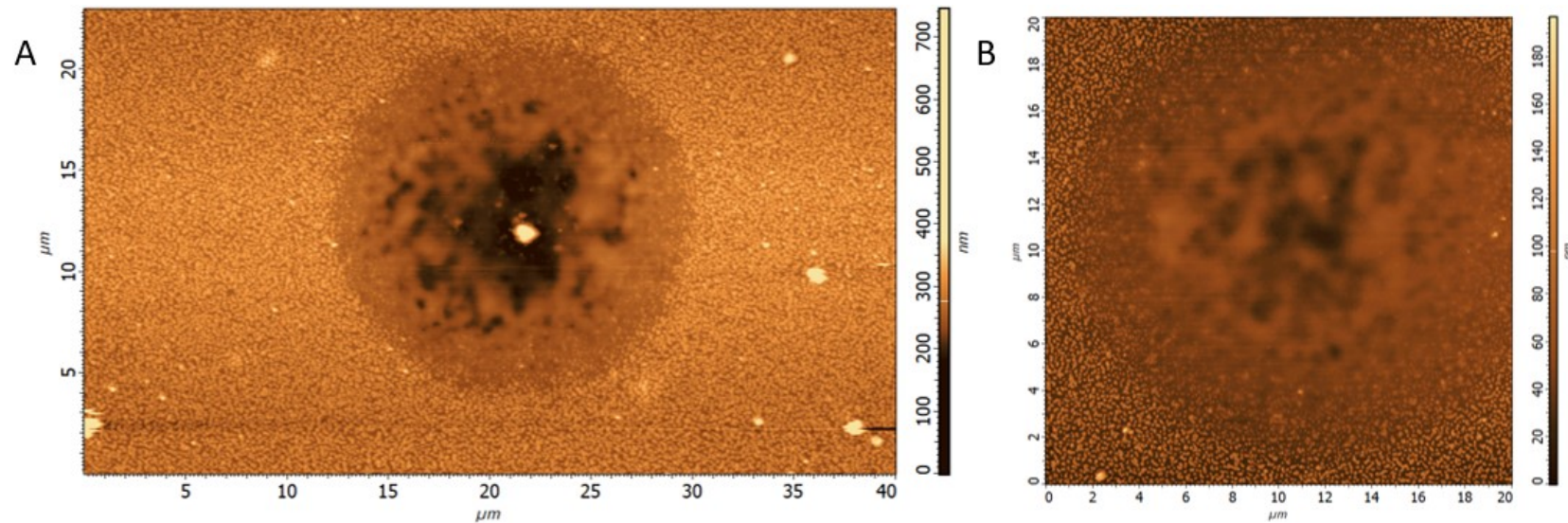

C
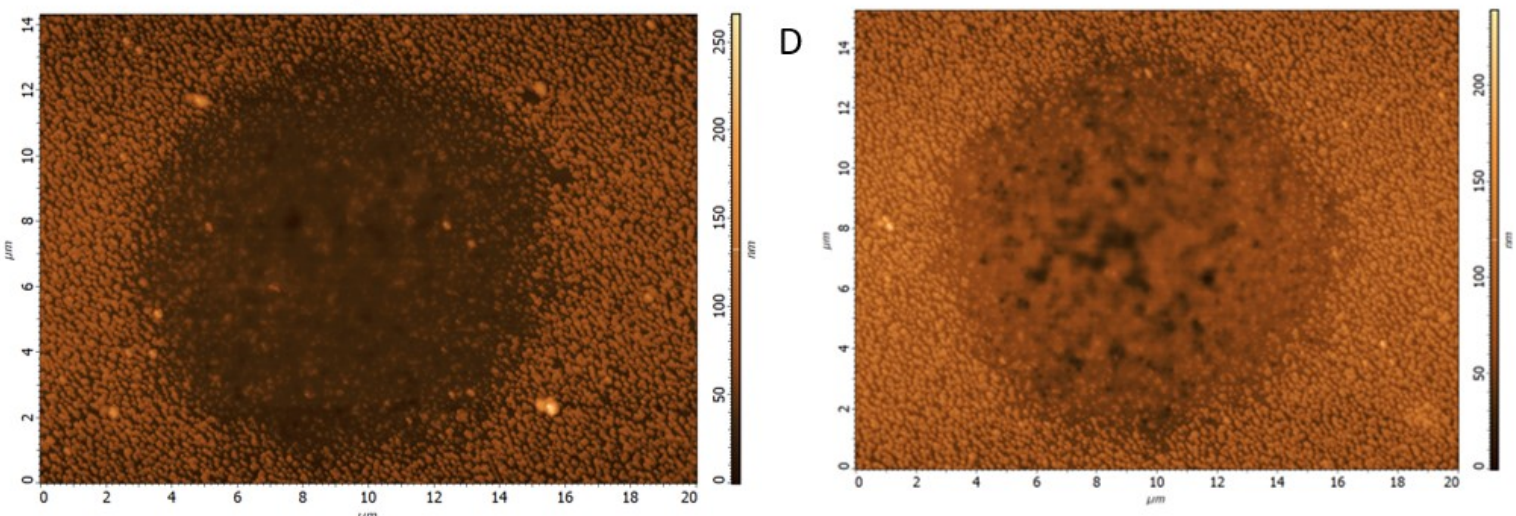

Figure 6.6: AFM images of AgNC monolayer after being exposed to a $458 \mathrm{~nm}$ laser at $40 \mathrm{~mW}$ for 15 second (A) and 5 seconds $(B)$ and at $23 \mathrm{~mW}$ for 15 seconds $(C)$ and 5 seconds (D). The diameters of the circular patterned regions were measured using Nova software to be approximately $18.24 \mu \mathrm{m}, 19.72 \mu \mathrm{m}, 13.05 \mu \mathrm{m}$ and 12.67 respectively. 

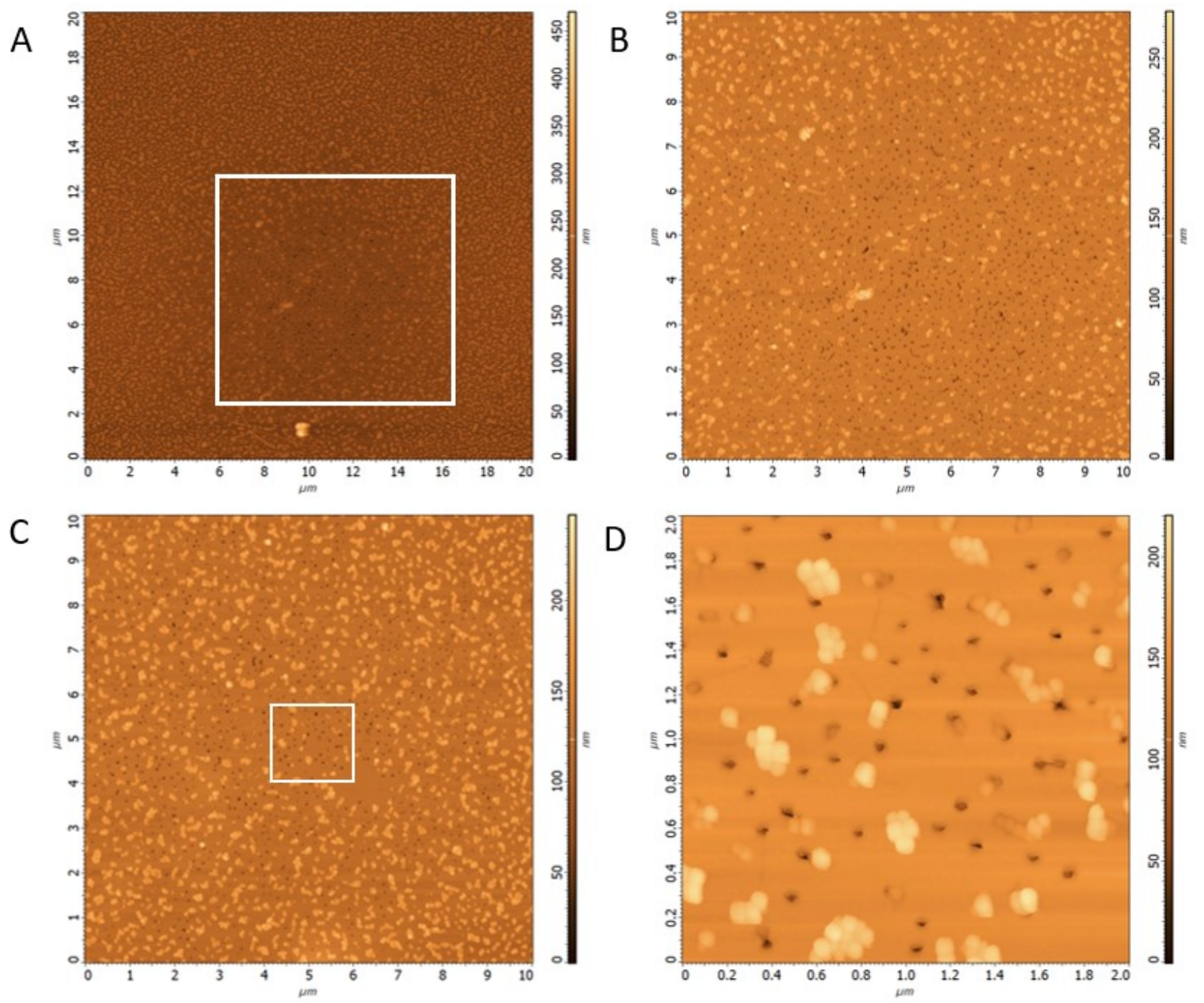

Figure 6.7: AFM images of AgNC monolayer after being exposed to a $458 \mathrm{~nm}$ laser at $17 \mathrm{~mW}$ for 5 minutes (A) and 3 minutes (C). $B$ and $D$ are zoomed in images of the regions outlined by the white square in $A$ and $C$ respectively. The diameter of patterned areas in A and C were measured with Nova to be $12.29 \mu \mathrm{m}(A)$ and $12.09 \mu \mathrm{m}(C)$.
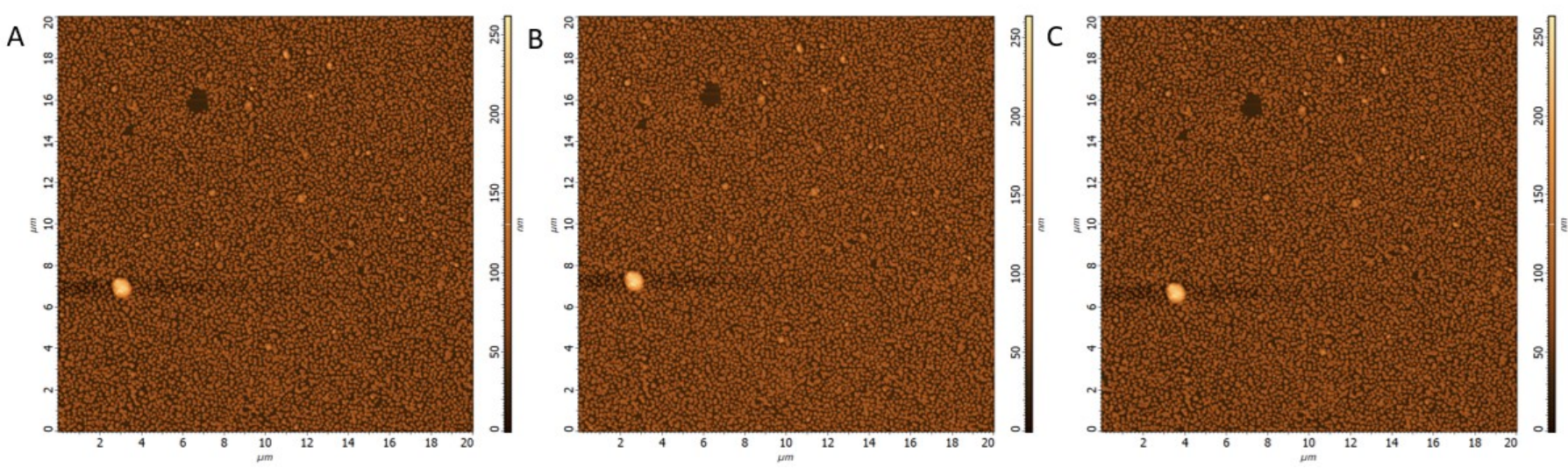

Figure 6.8: AFM images of AgNC monolayer after being exposed to a $568 \mathrm{~nm}$ laser at $50 \mathrm{~mW}$ for 30 seconds (A), an additional 2 minutes (B) and an additional 5 minutes (C). Even after 7 minutes and 30 seconds of exposure at $50 \mathrm{~mW}$ there was no patterning observed. 
A

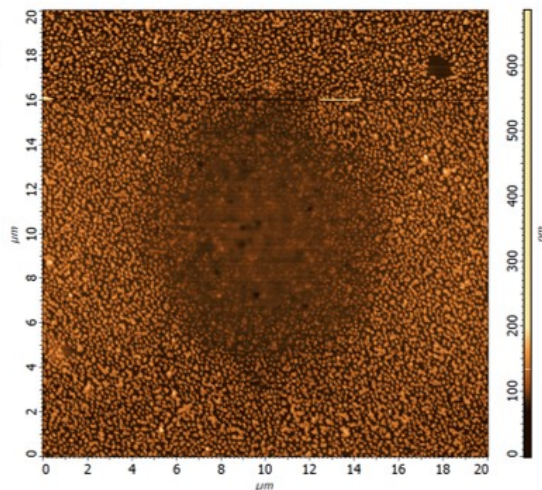

D

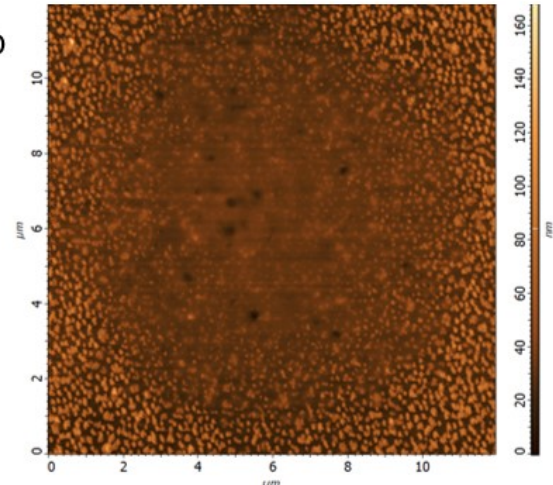

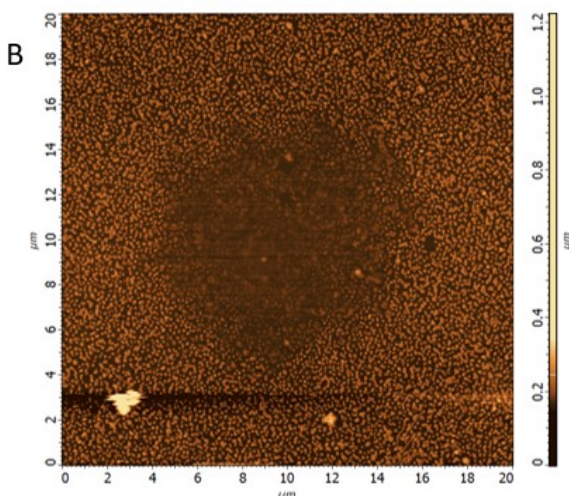

E

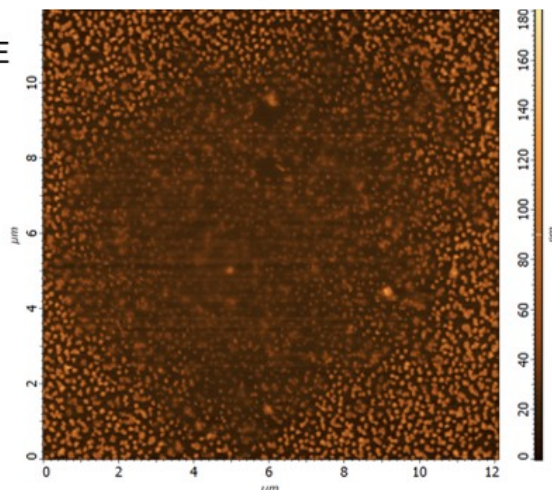

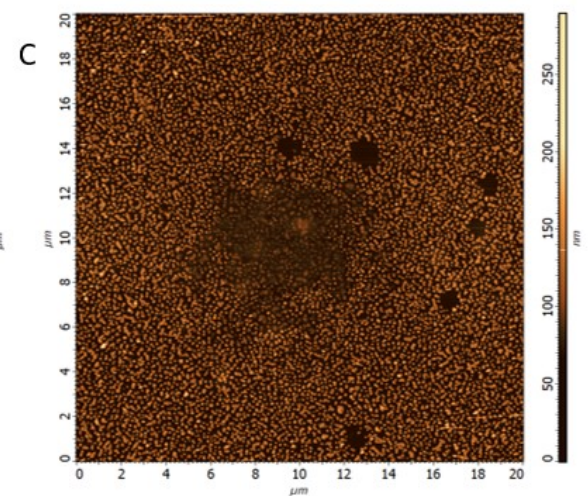

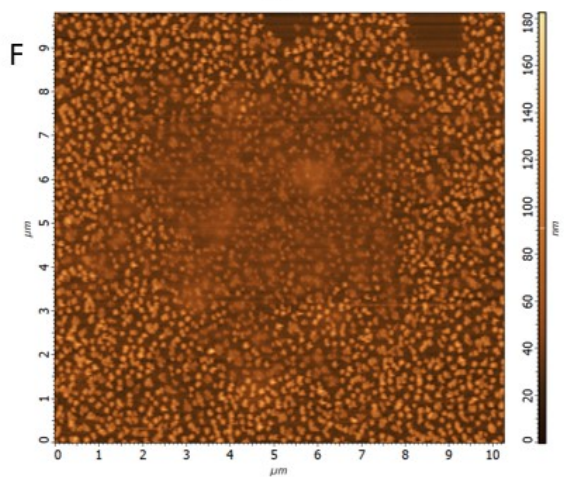

Figure 6.9: AFM images of AgNC monolayer after being exposed to a $568 \mathrm{~nm}$ laser at $65 \mathrm{~mW}$ for 2 minutes (A), 1 minute (B) and 20 seconds $(C)$. D, E and $F$ are zoomed in images of the partially embedded regions in $A, B$ and $C$ respectively. The diameters of the circular patterned regions were measured using Nova software to be approximately $11.18 \mu \mathrm{m}(\mathrm{A}), 11.13 \mu \mathrm{m}$ (B) and $8.26 \mu \mathrm{m}(C)$. 
6.3 Supplementary Material for Section 3.3

6.3.1 Series of AFM Images Taken Before and After Laser Exposure at $458 \mathrm{~nm}$

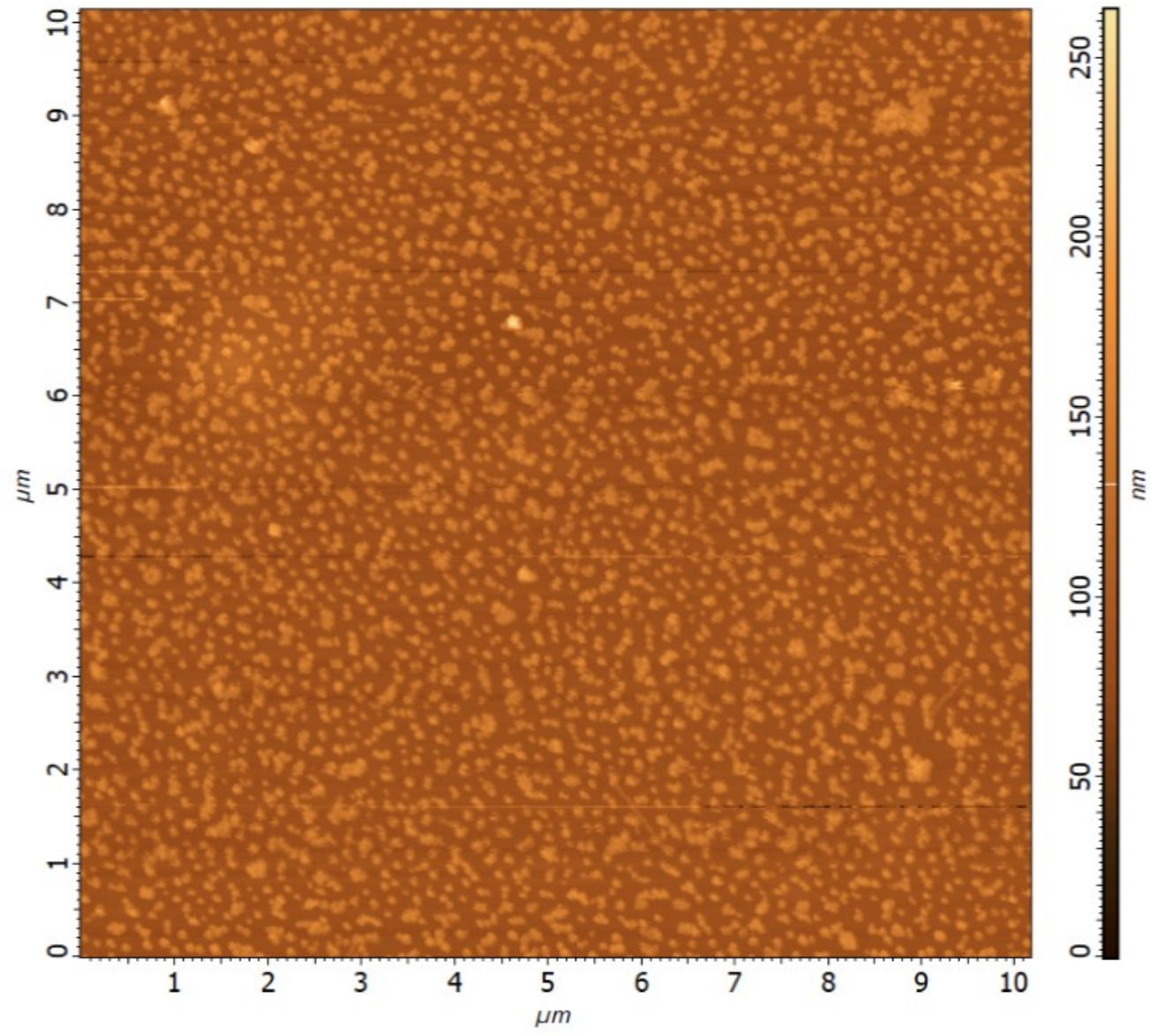

Figure 6.10: $10 \mu \mathrm{m} \times 10 \mu \mathrm{m}$ AFM of AgNC monolayer used for selective embedment experiments of individual cubes before laser exposure. 


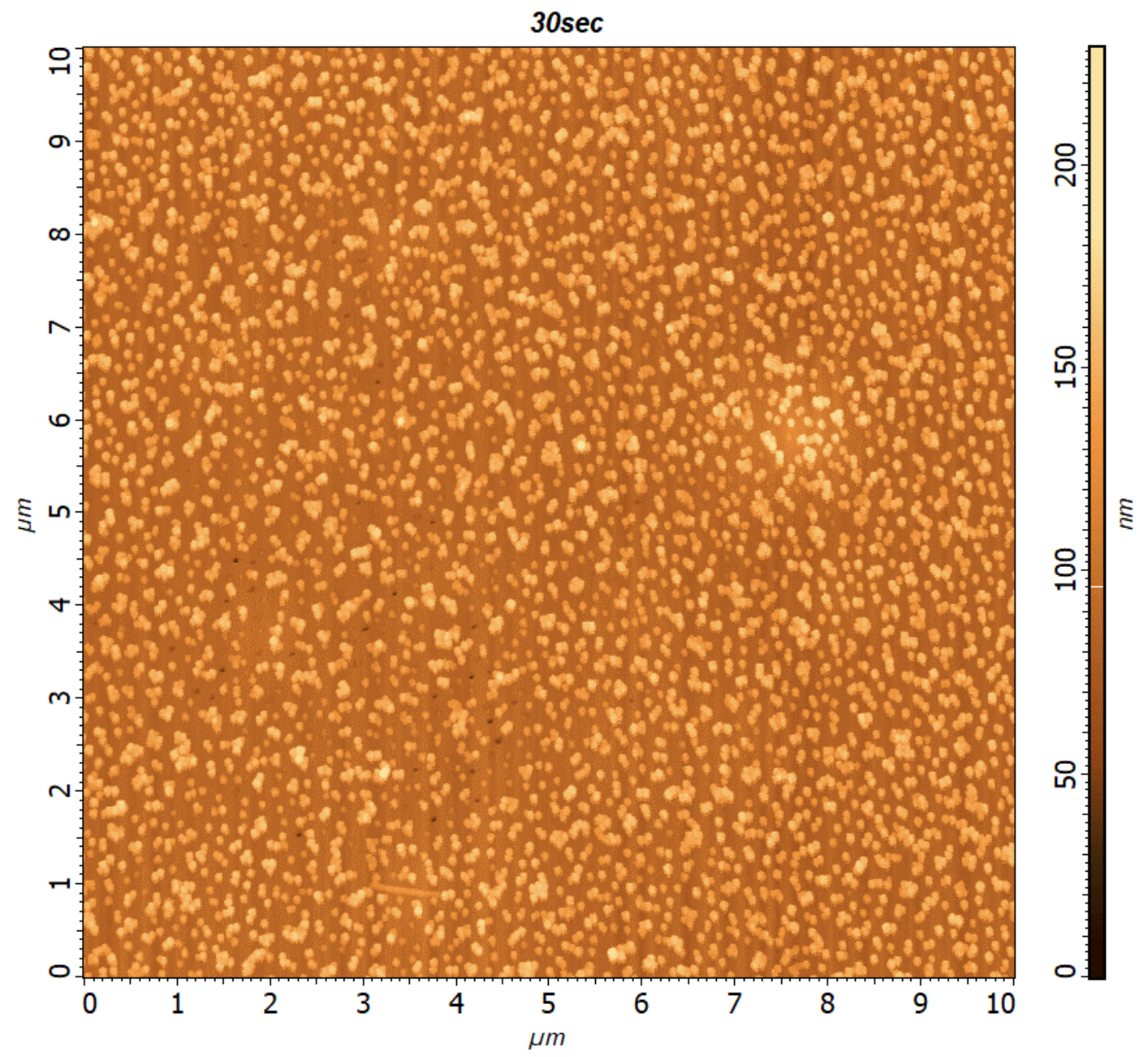

Figure 6.11: $10 \mu \mathrm{m} \times 10 \mu \mathrm{m}$ AFM of AgNC monolayer used for selective embedment experiments of individual cubes after 30s of laser exposure. 


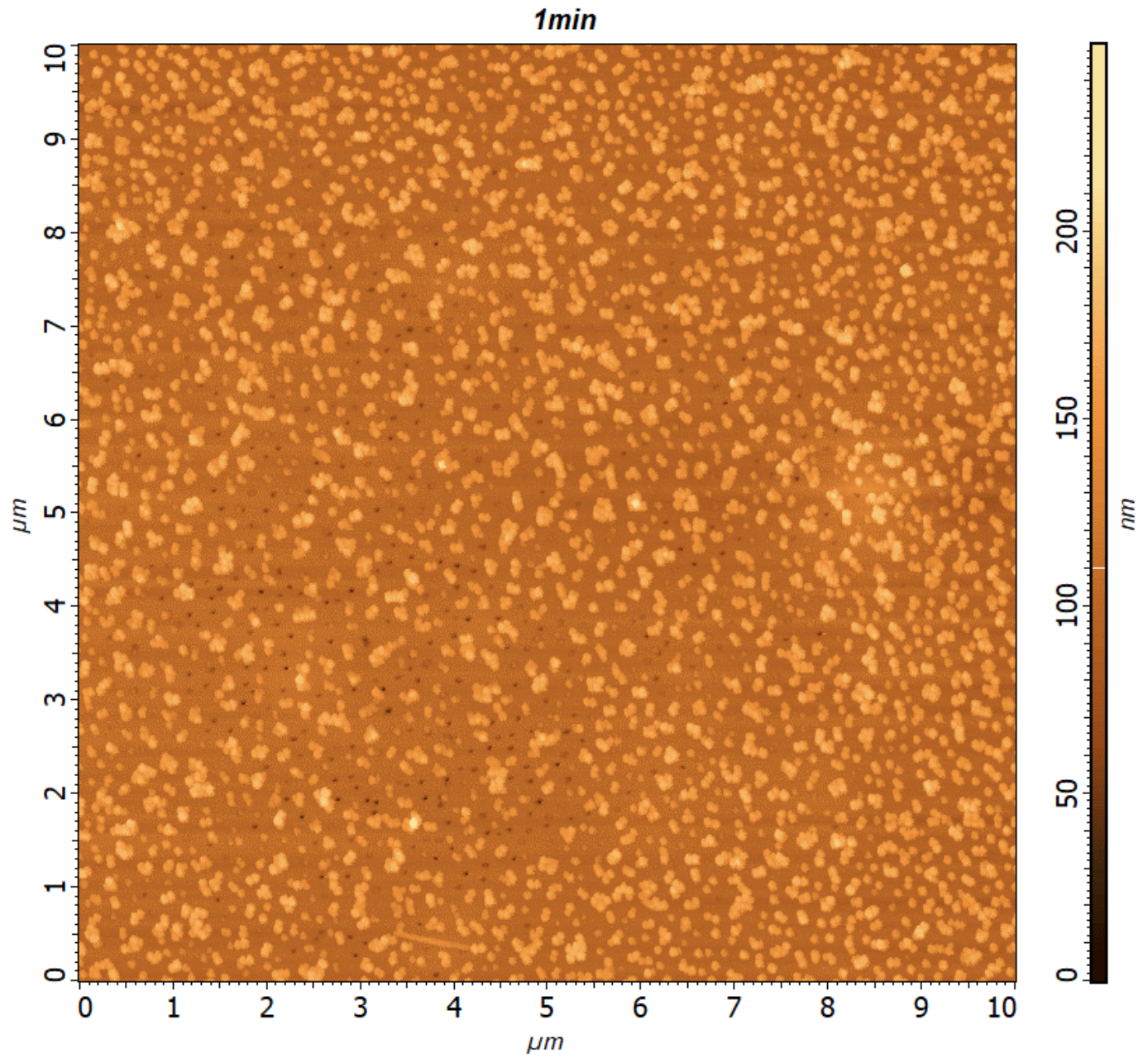

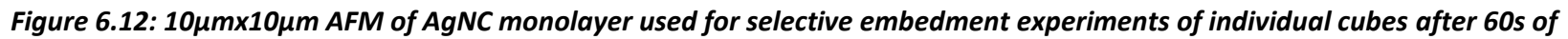
laser exposure. 


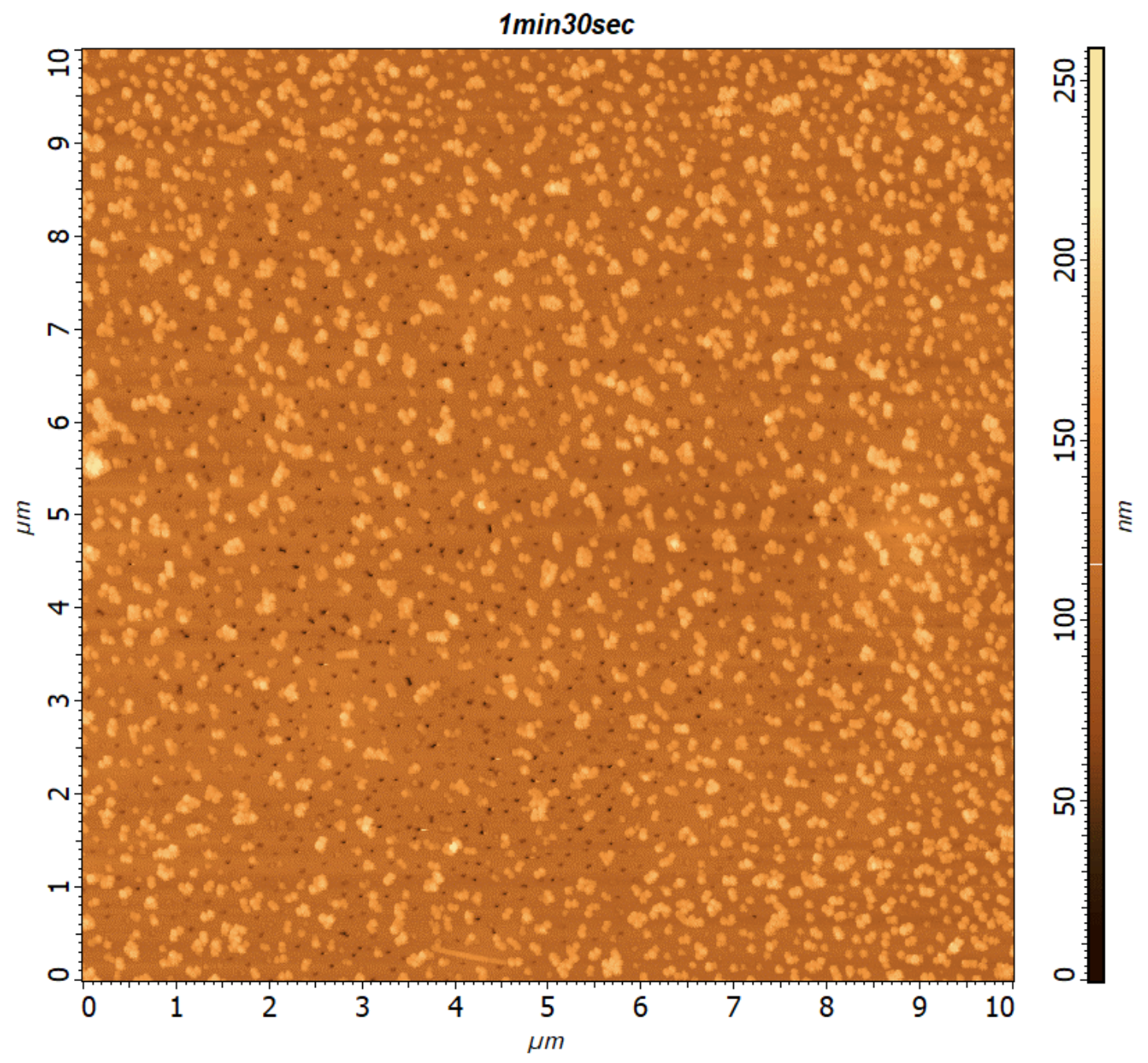

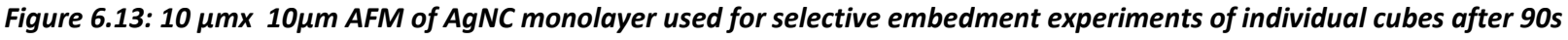
of laser exposure. 


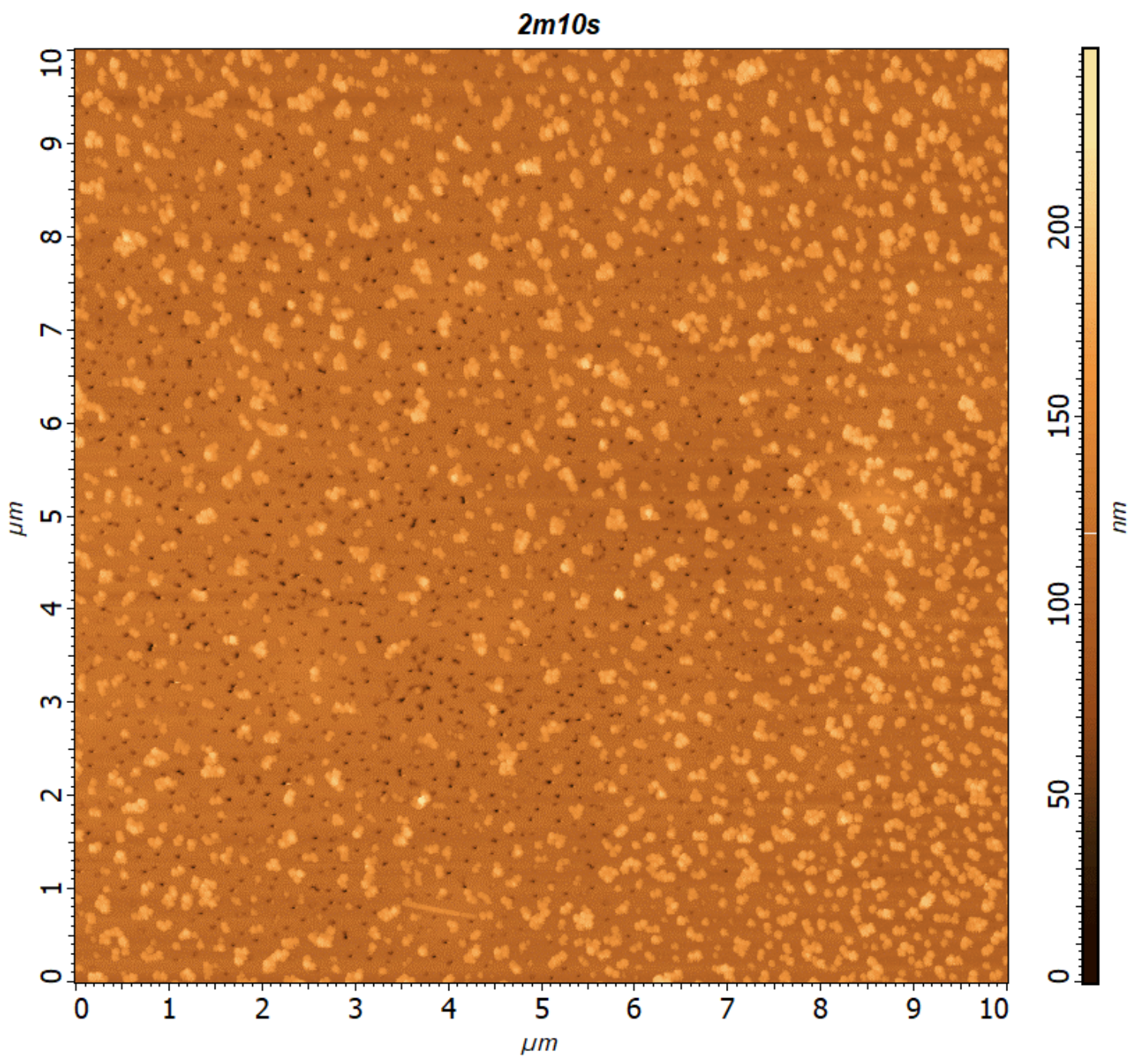

Figure 6.14: $10 \mu \mathrm{m} \times 10 \mu \mathrm{m}$ AFM of AgNC monolayer used for selective embedment experiments of individual cubes after 130 s of laser exposure. 


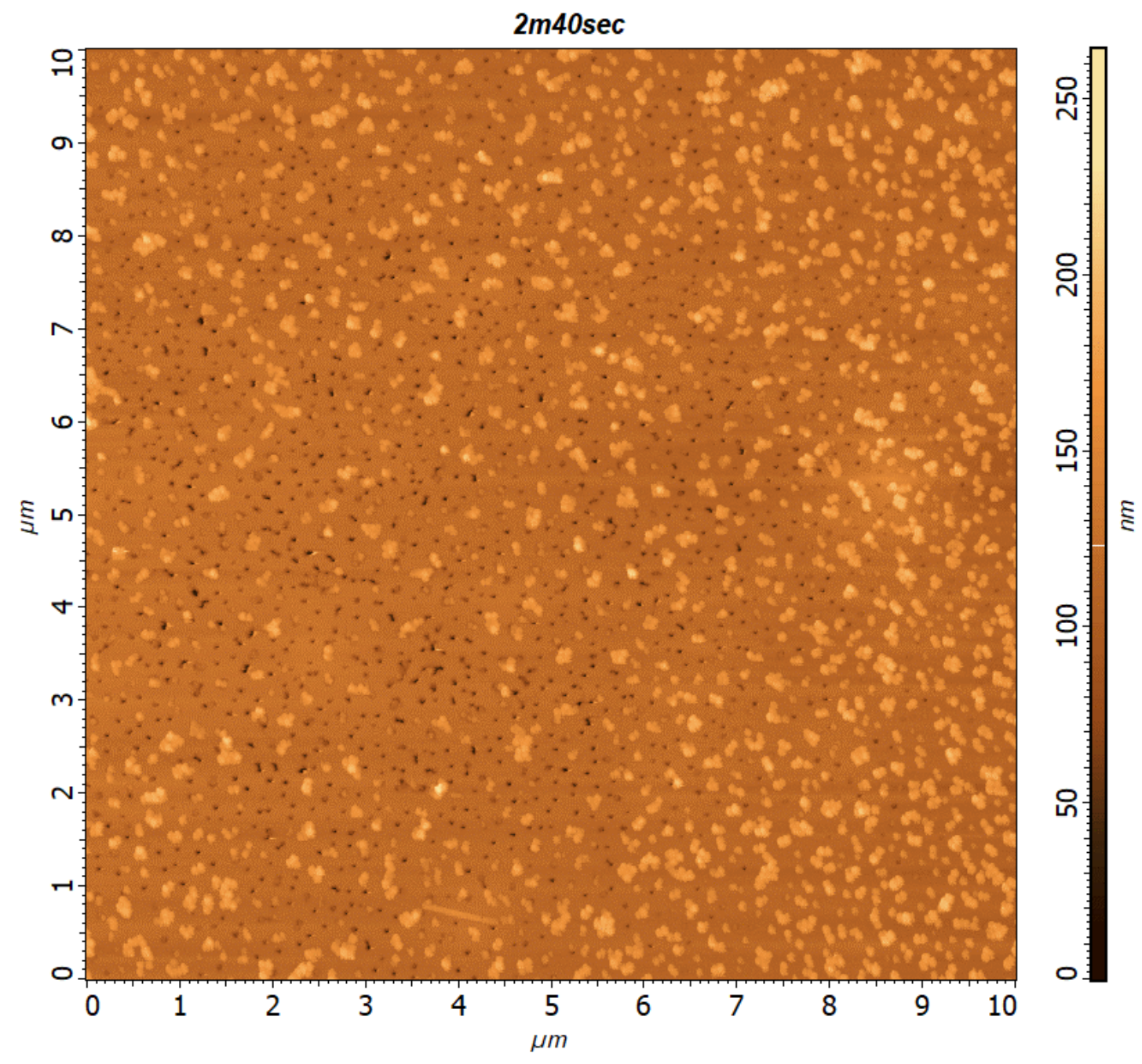

Figure 6.15: $10 \mu \mathrm{m} \times 10 \mu \mathrm{m}$ AFM of AgNC monolayer used for selective embedment experiments of individual cubes after 160 s of laser exposure. 


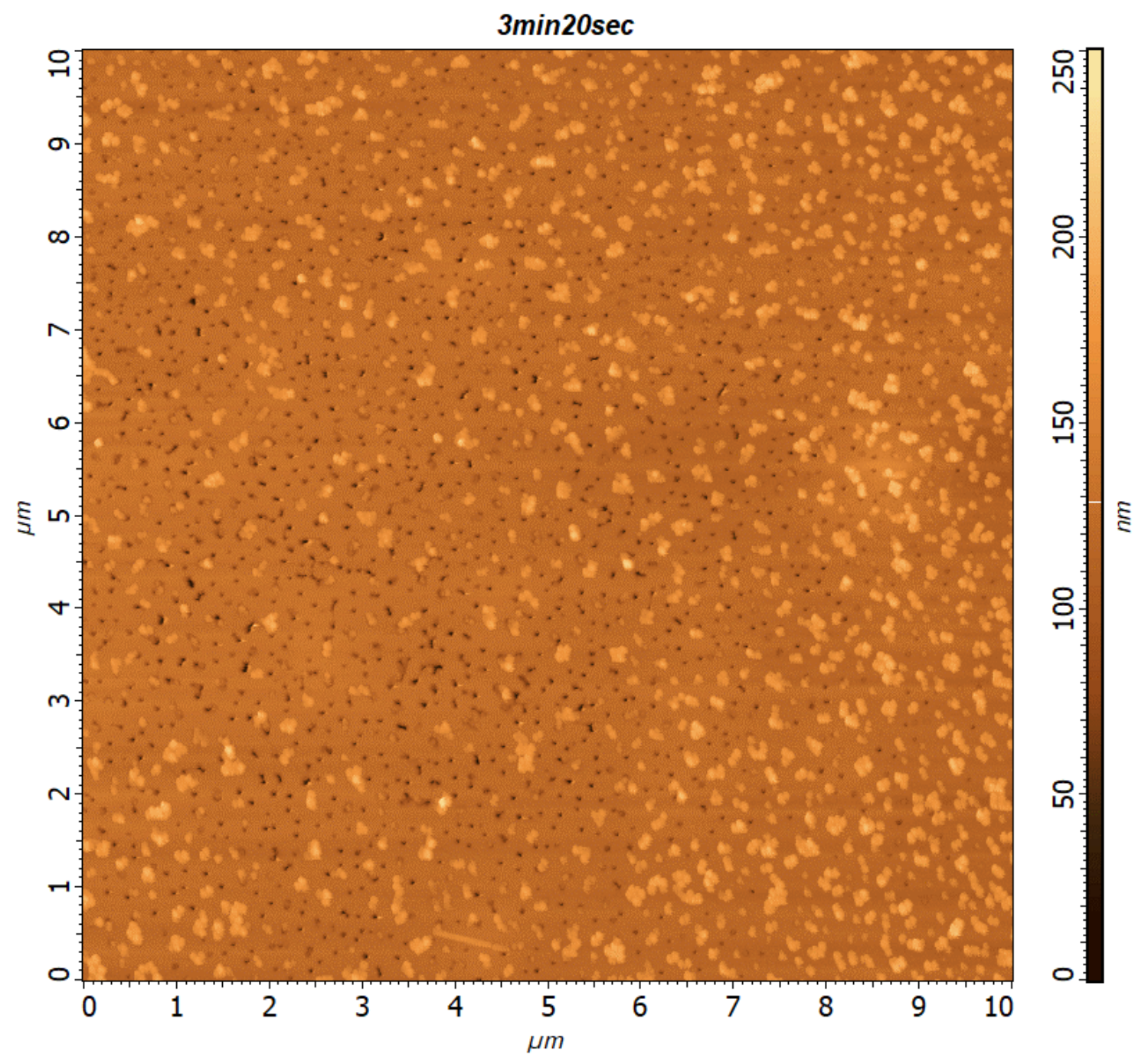

Figure 6.16: $10 \mu \mathrm{m} \times 10 \mu \mathrm{m}$ AFM of AgNC monolayer used for selective embedment experiments of individual cubes after 200s of laser exposure. 


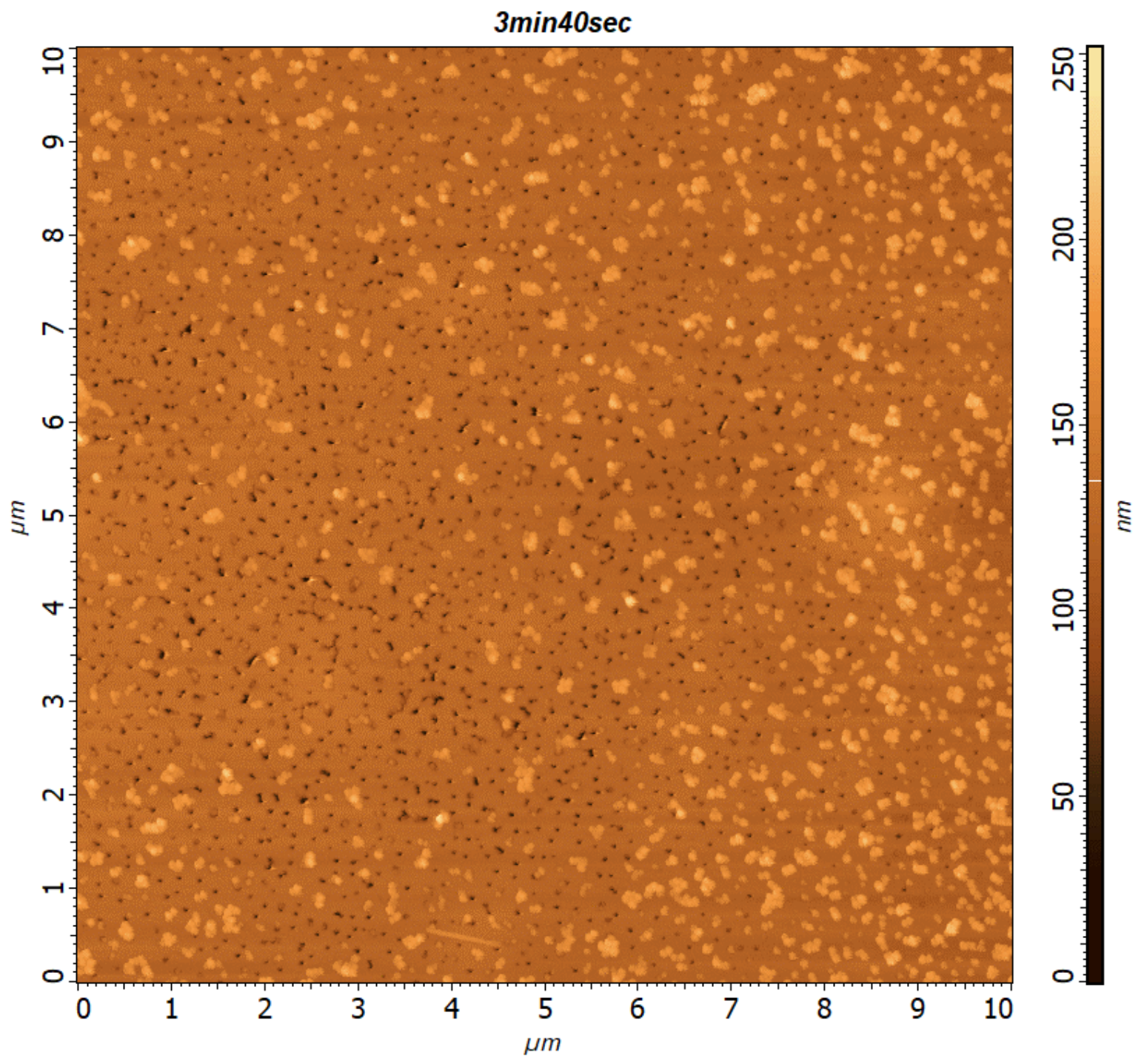

Figure 6.17: $10 \mu \mathrm{m} \times 10 \mu \mathrm{m}$ AFM of AgNC monolayer used for selective embedment experiments of individual cubes after 220 s of laser exposure. 


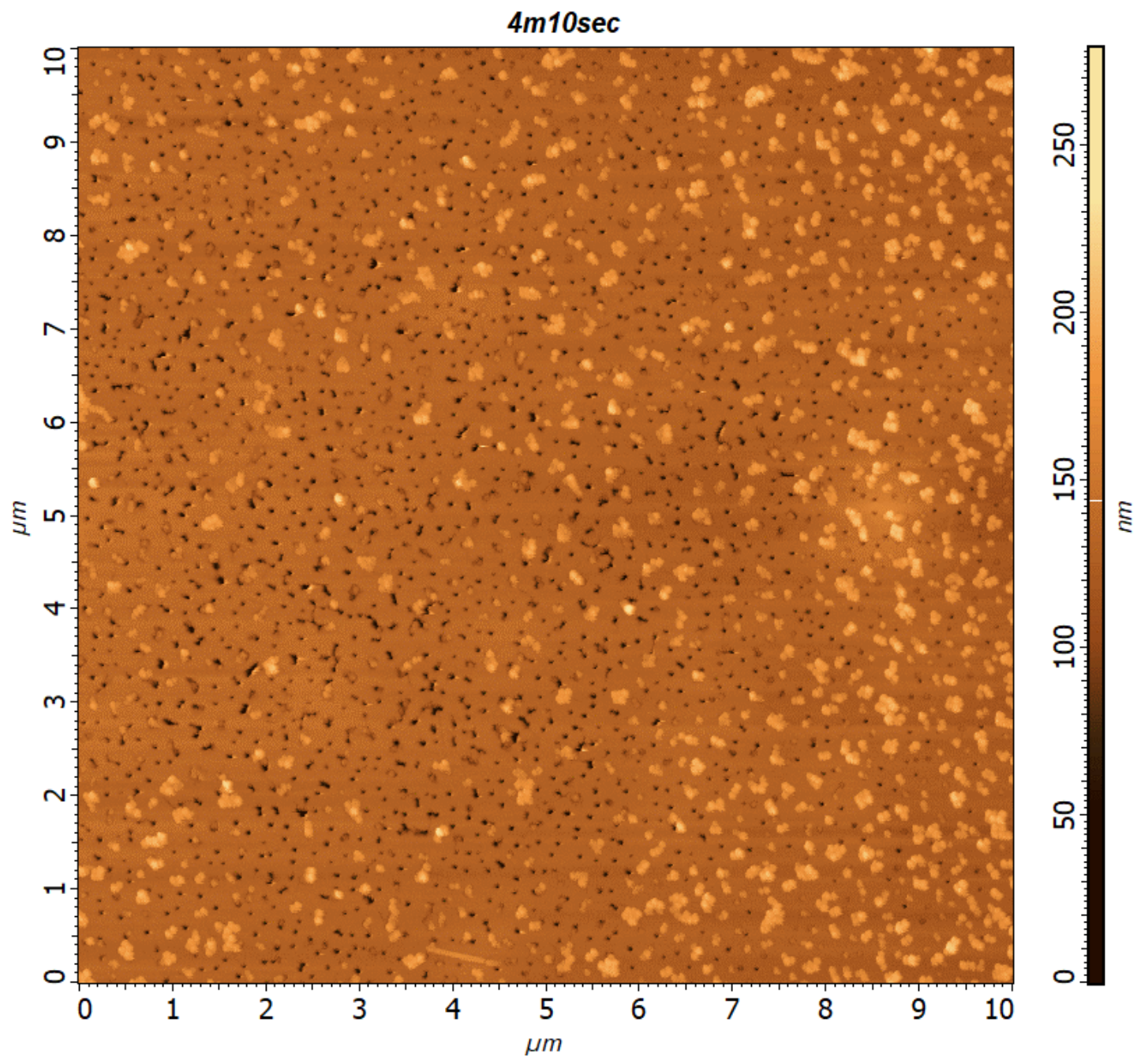

Figure 6.18: $10 \mu \mathrm{m} \times 10 \mu \mathrm{m}$ AFM of AgNC monolayer used for selective embedment experiments of individual cubes after 250s of laser exposure. 


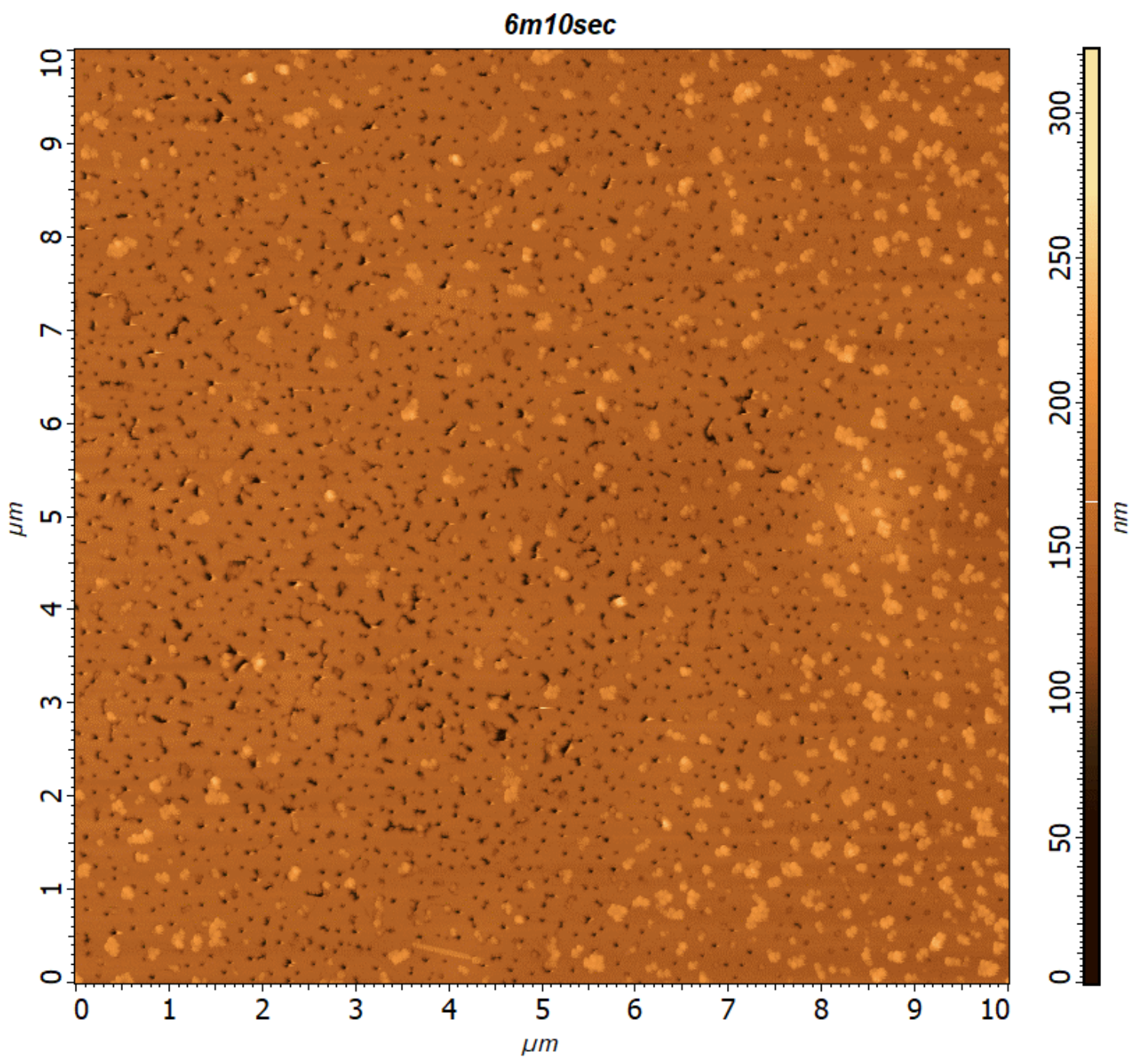

Figure 6.19: $10 \mu \mathrm{m} \times 10 \mu \mathrm{m}$ AFM of AgNC monolayer used for selective embedment experiments of individual cubes after 370 s of laser exposure. 


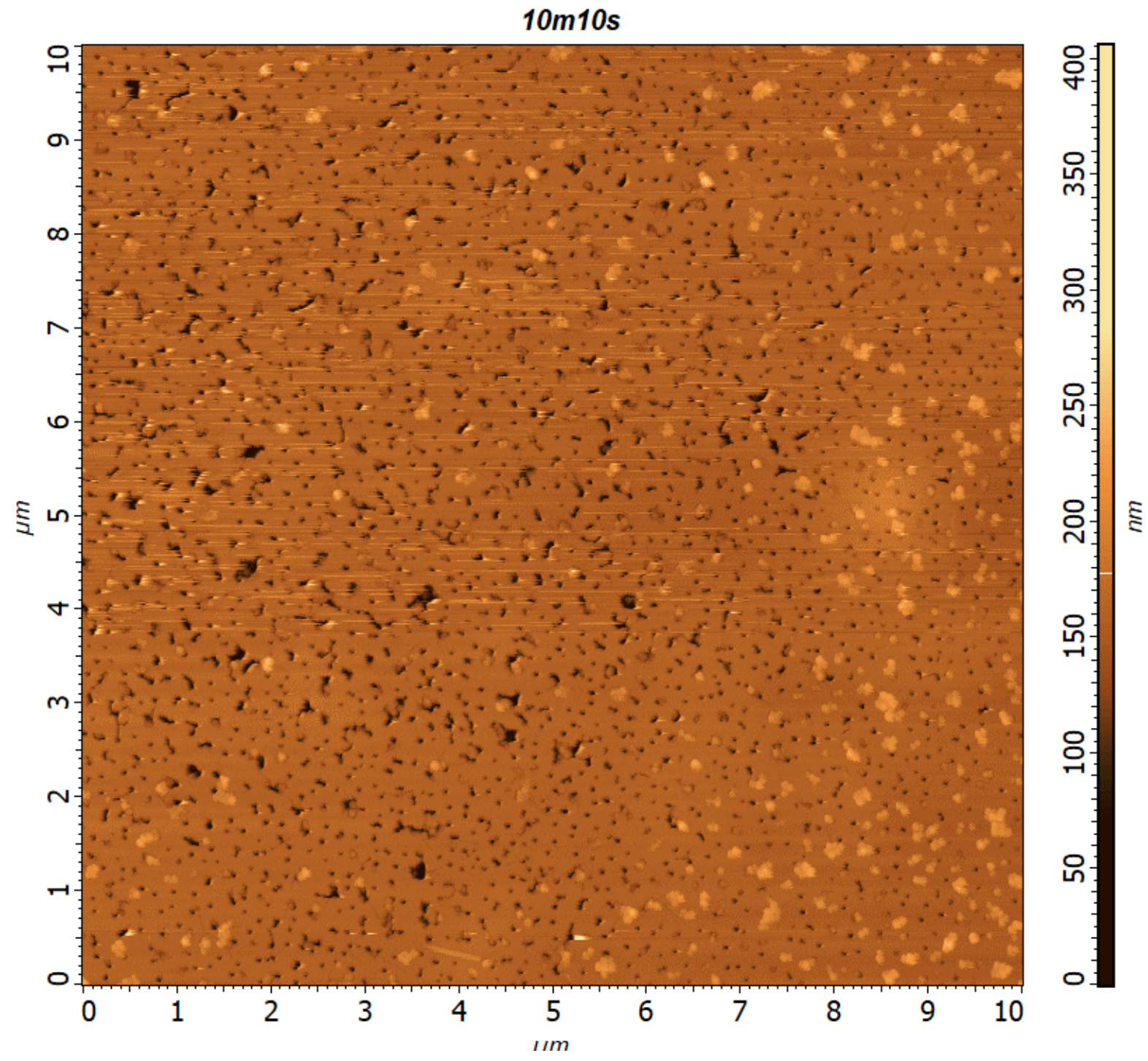

Figure 6.20: $10 \mu \mathrm{m} \times 10 \mu \mathrm{m}$ AFM of AgNC monolayer used for selective embedment experiments of individual cubes after 610s of laser exposure. 


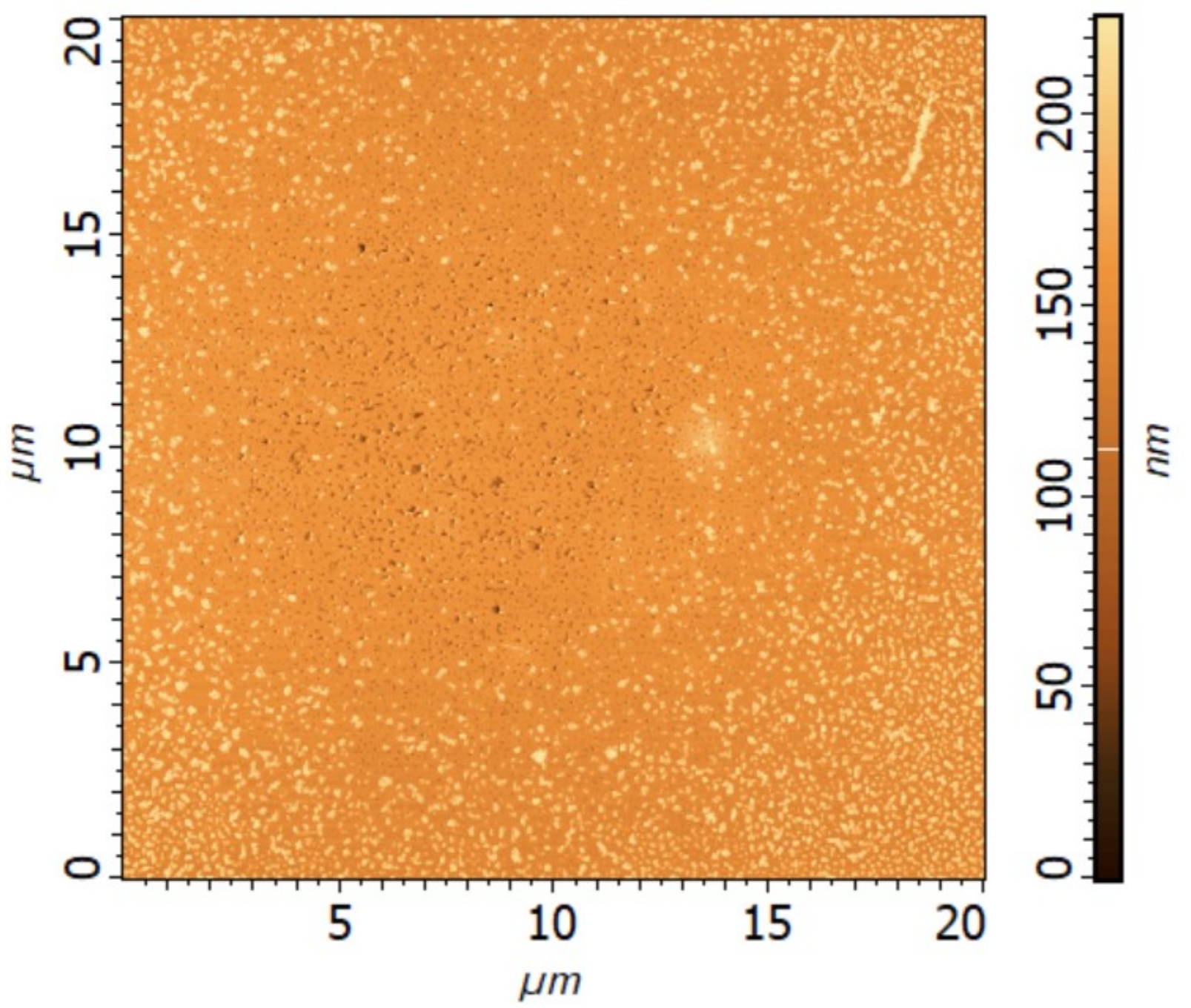

Figure 6.21: $20 \mu \mathrm{m} \times 20 \mu \mathrm{m}$ AFM of AgNC monolayer after being exposed to $458 \mathrm{~nm}$ laser for 610s. The diameter of the circular pattern was measured with Nova to be $17.13 \mu \mathrm{m}$. 
6.3.2 Zoomed in Regions of AFM images Taken After Laser Embedment Intervals

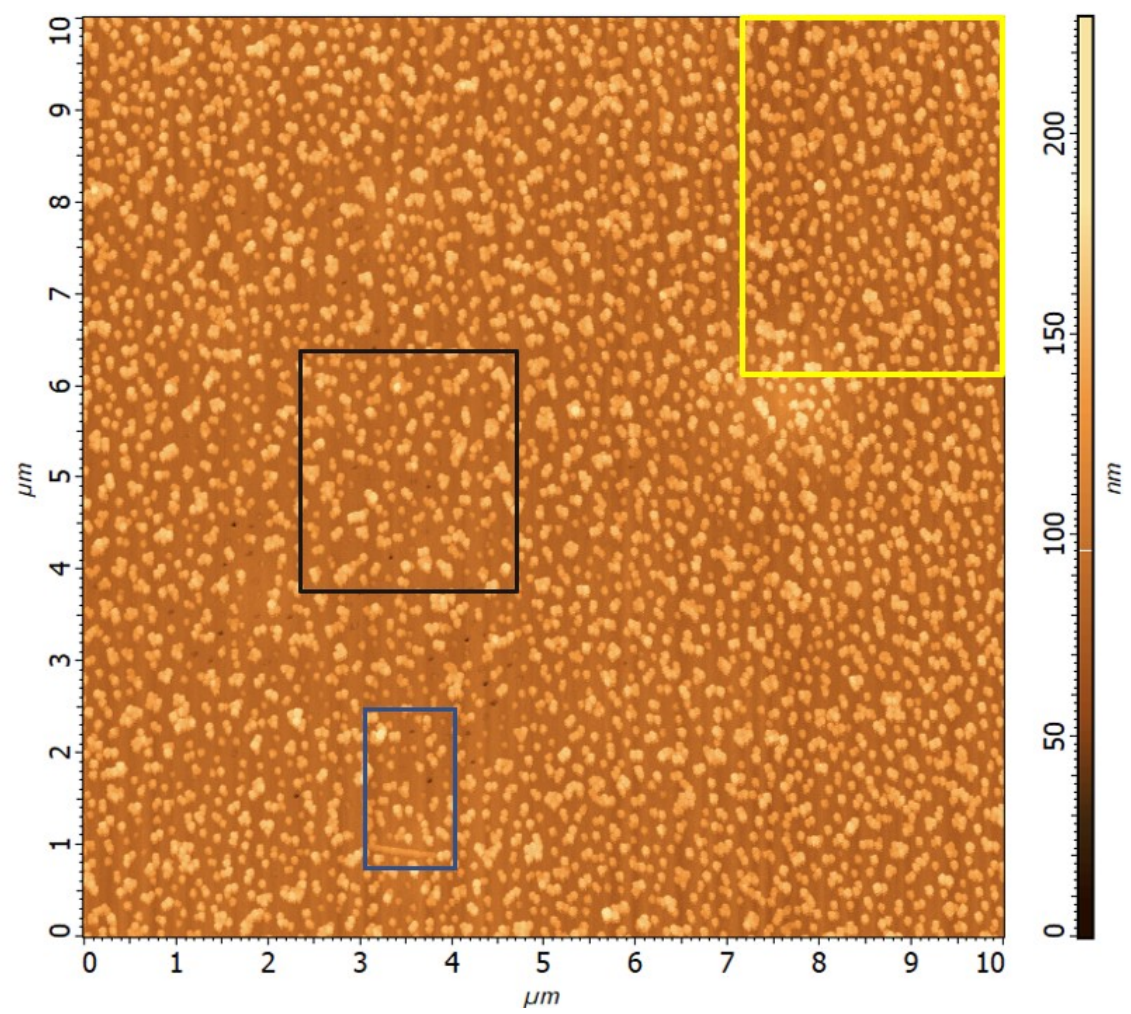

Figure 6.22: Outline of different regions of original AFM images used for zoomed-In images in Figures 6.23-6.25. The black, blue and yellow rectangles outline Region I, II and III respectively. The regions are shown here using the $10 \mu \mathrm{m} \times 10 \mu \mathrm{m}$ AFM taken 30 s after laser exposure for reference. 

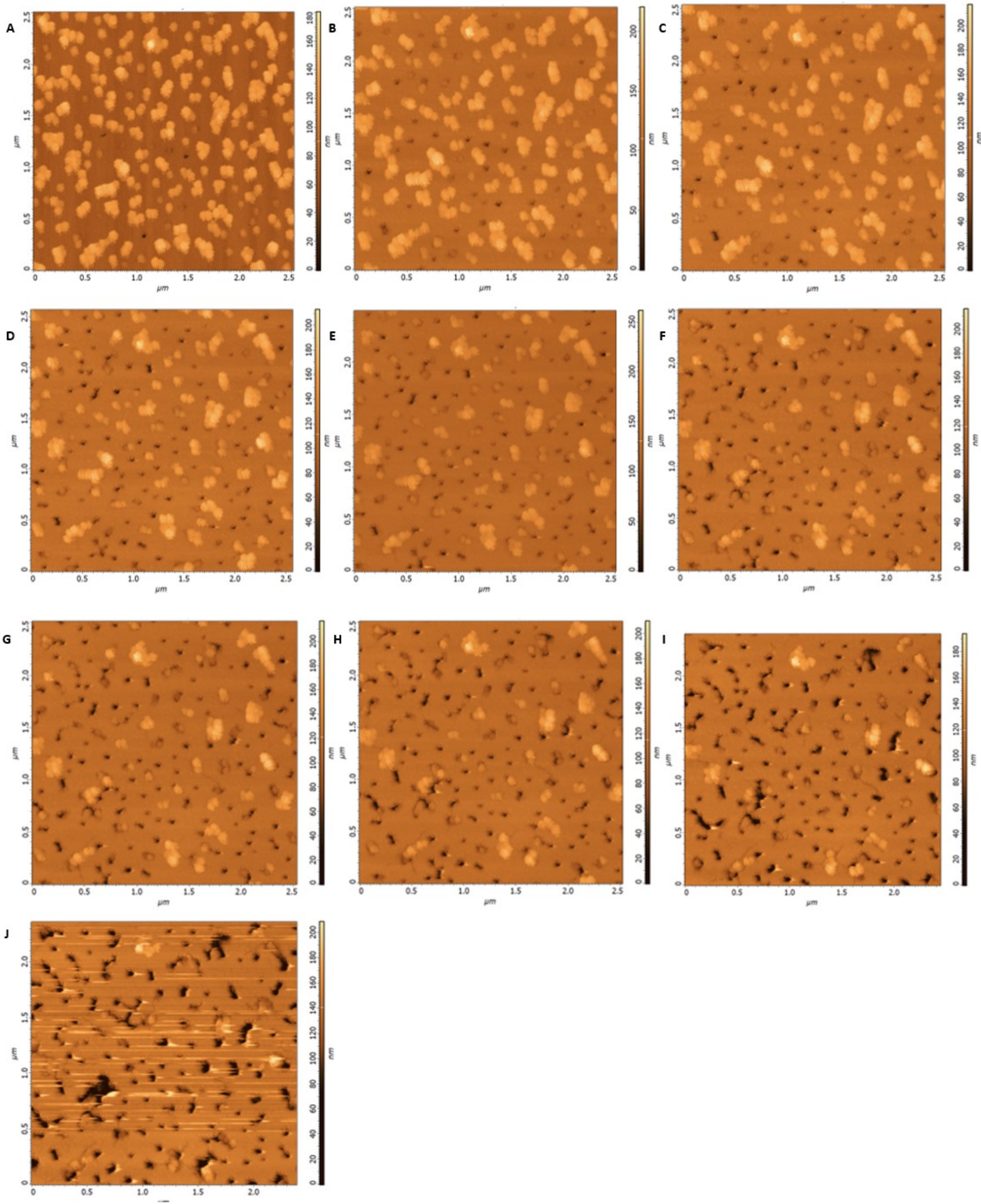

Figure 6.23: Close-up of Region I following 30s, 60s, 90s (A-C), 130s, 160s, 200s (D-F), 220s, 250s, 370s (G-I), and 610s (J) of exposure with the $458 \mathrm{~nm}$ laser. 

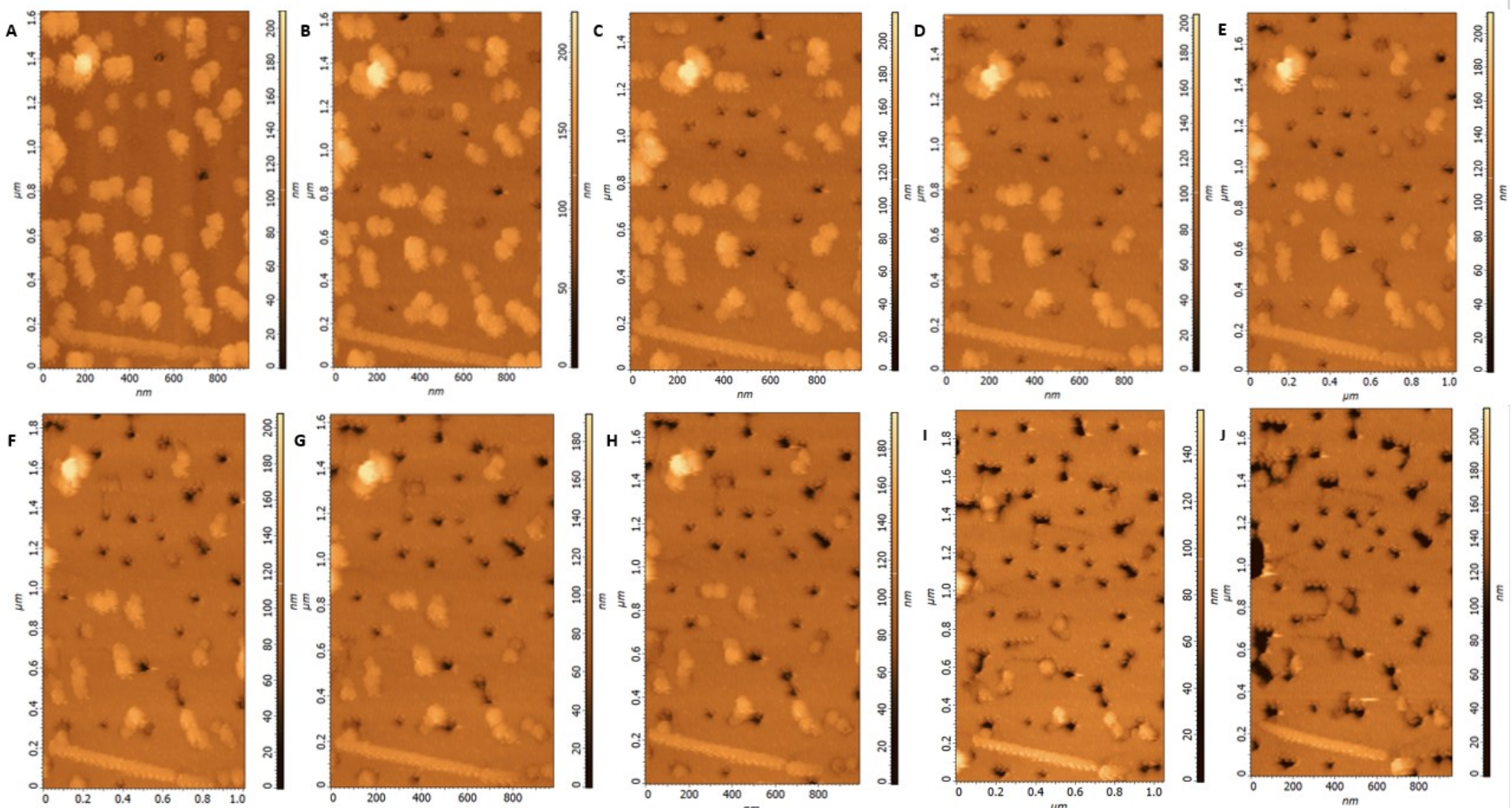

Figure 6.24: Close-up of Region II following 30s, 60s, 90s, 130s, 160s (A-E), 200s, 220s, 250s, 370s and 610s (F-J) of exposure with the $458 \mathrm{~nm}$ laser.
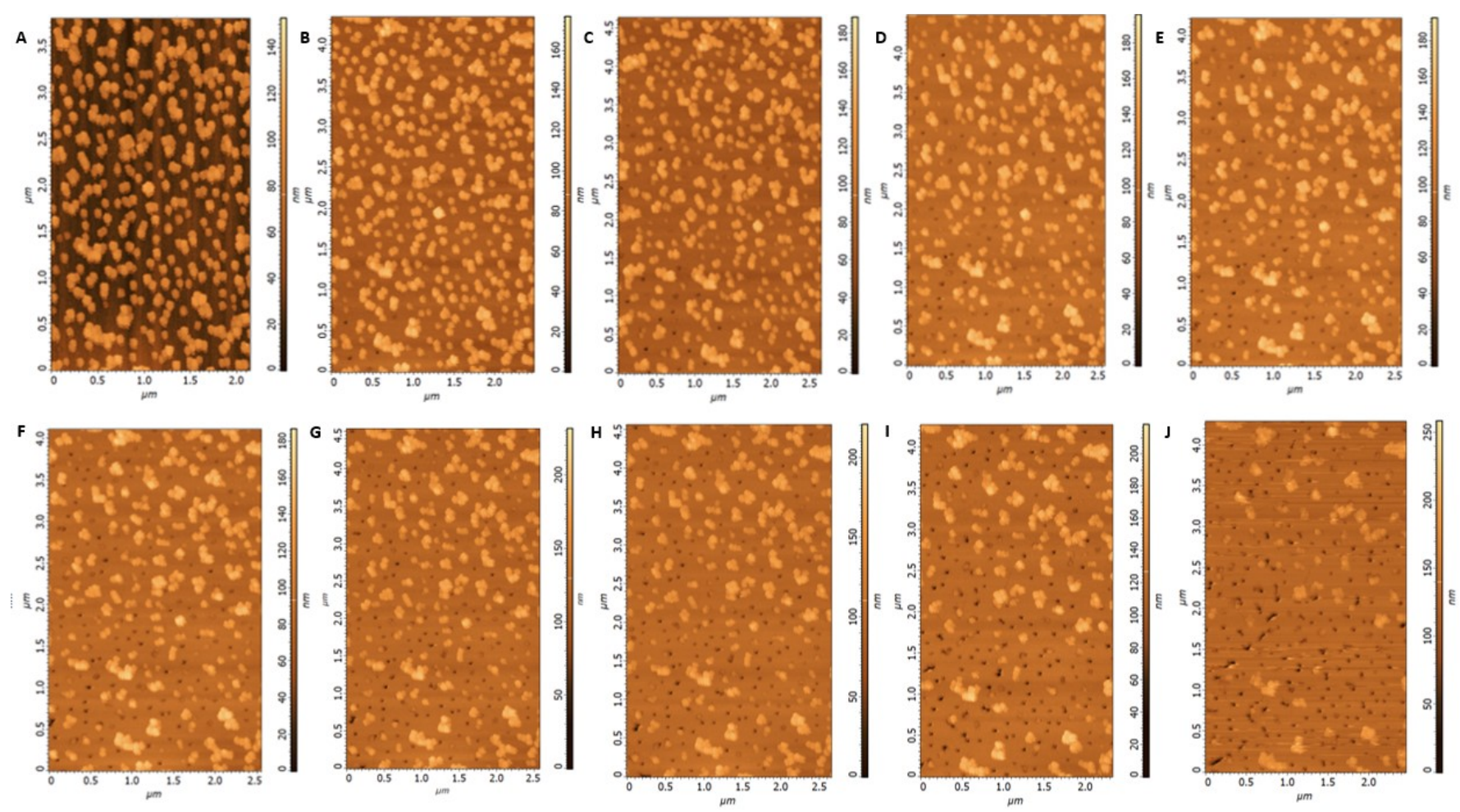

Figure 6.25: Close-up of Region III following 30s, 60s, 90s, 130s, 160s (A-E), 200s, 220s, 250s, 370s and 610s (F-J) of exposure with the $458 \mathrm{~nm}$ laser. 


\subsection{Supplementary Material for Section 3.4}

6.4.1 Regions of the AgNC Monolayer Investigated for Selective Embedment of Nanocube Clusters at $568 \mathrm{~nm}$
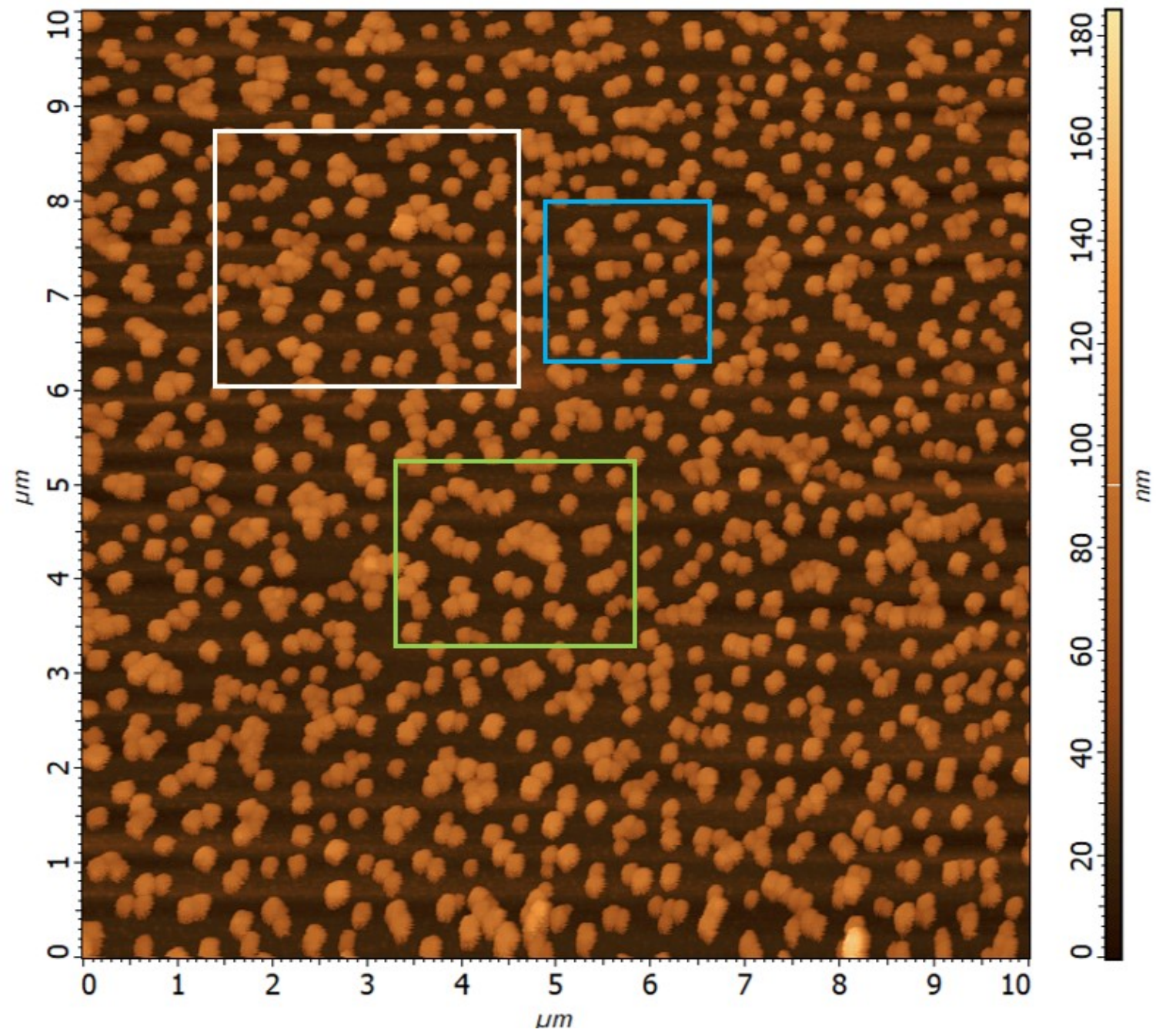

Figure 6.26: Outline of different regions used for zoomed-In images in Figures 6.27-6.29. The blue, green and white rectangle outline Regions A, B and C respectively. The regions are shown here using the $10 \mu \mathrm{m} \times 10 \mu \mathrm{m}$ AFM taken before laser exposure for reference. 

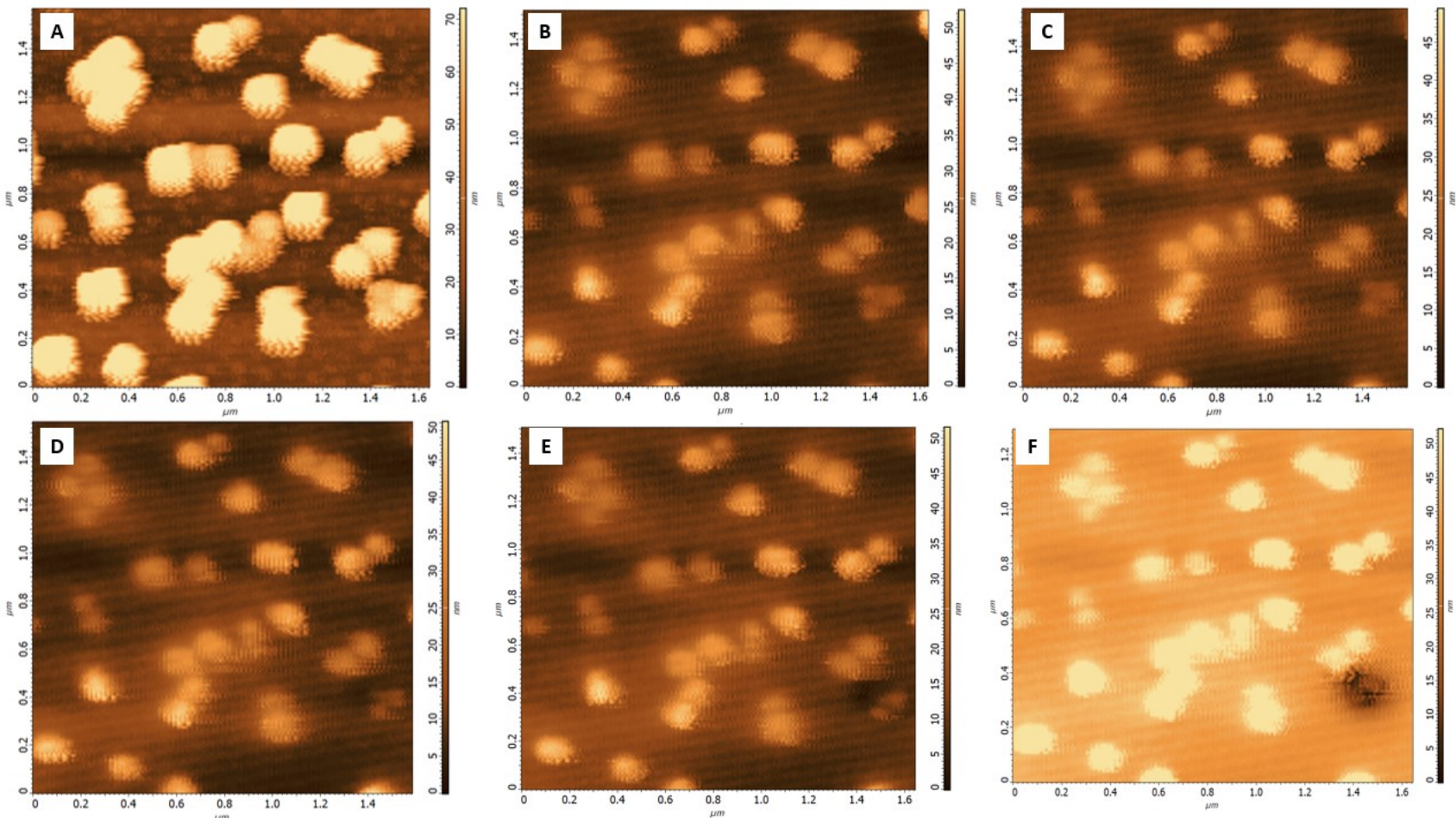

Figure 6.27: Close-up AFM images of Region A before laser exposure (A), and after 30s (B), 60s (C), 90s (D), 120s (E) and 180s (F) of exposure to the $568 \mathrm{~nm}$ laser.
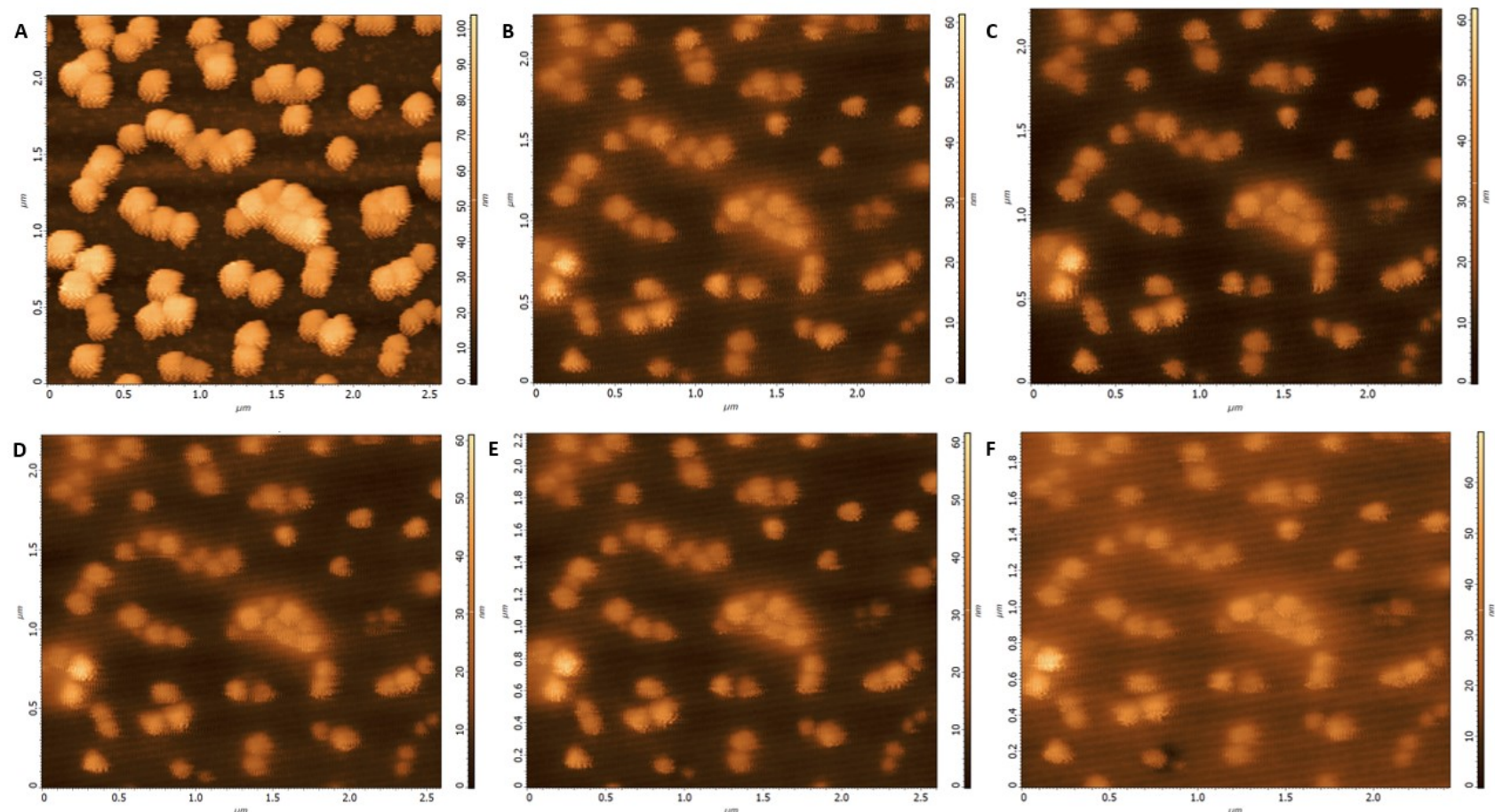

Figure 6.28: Close-up AFM images of Region B before laser exposure (A), and after 30s (B), 60s (C), 90s (D), 120s (E) and 180s (F) of exposure to the $568 \mathrm{~nm}$ laser. 
A

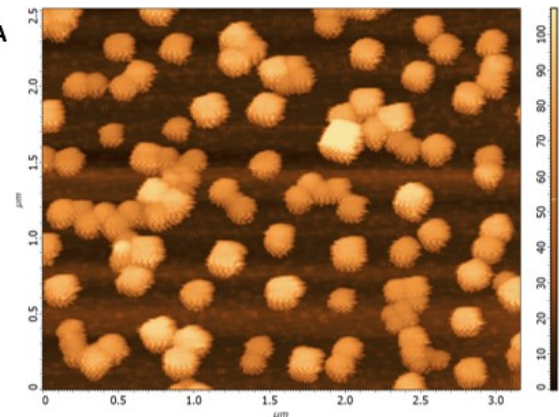

D

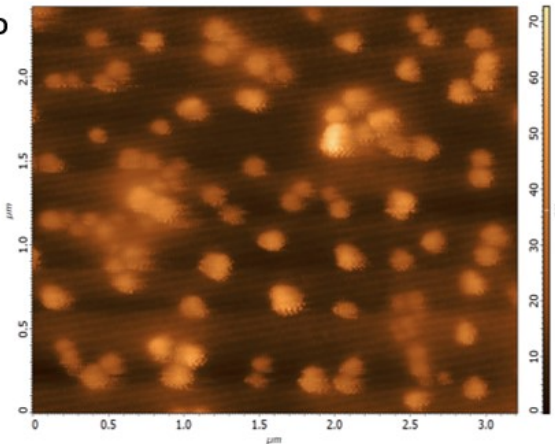

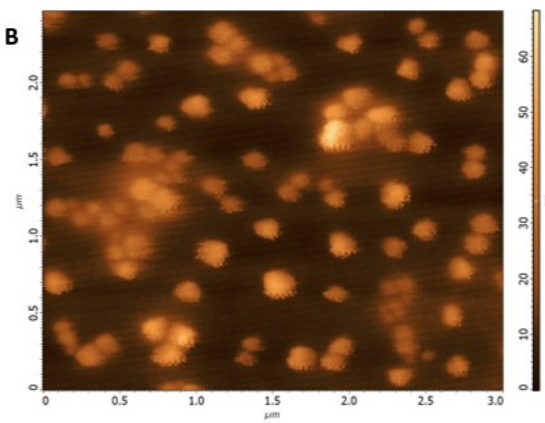

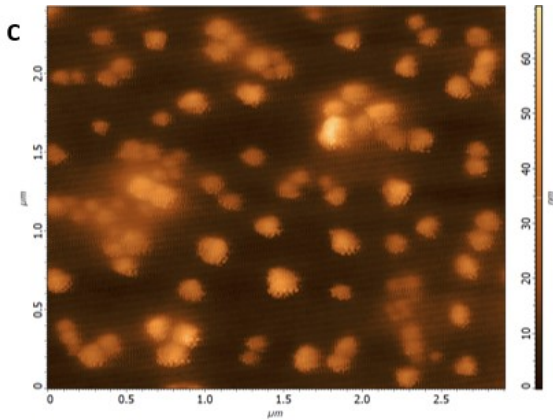

E
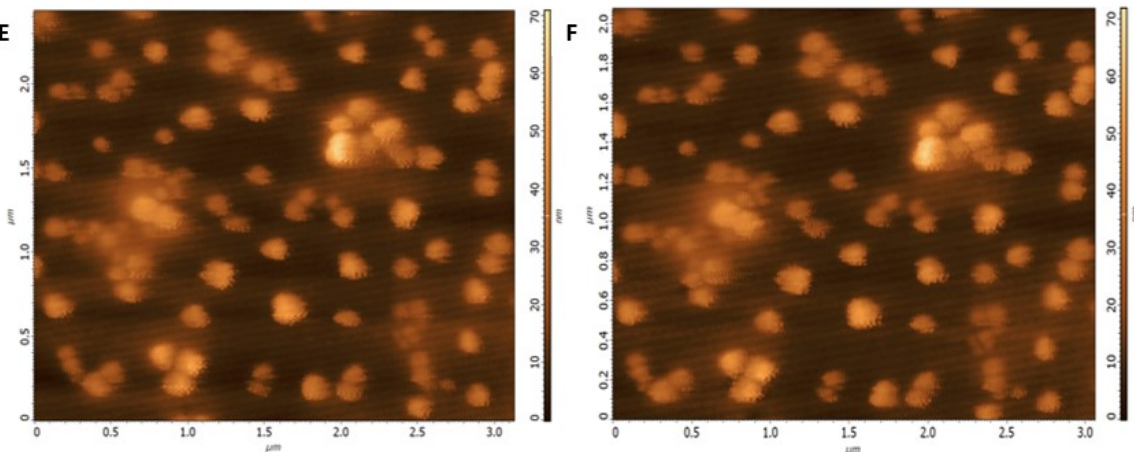

Figure 6.29: Close-up AFM images of Region C before laser exposure (A), and after 30s (B), 60s (C), 90s (D), 120s (E) and 180s (F) of exposure to the $568 \mathrm{~nm}$ laser 


\subsubsection{Cross Sections of AgNC Monolayer in Regions A, B and C used for Height} Measurement Correction
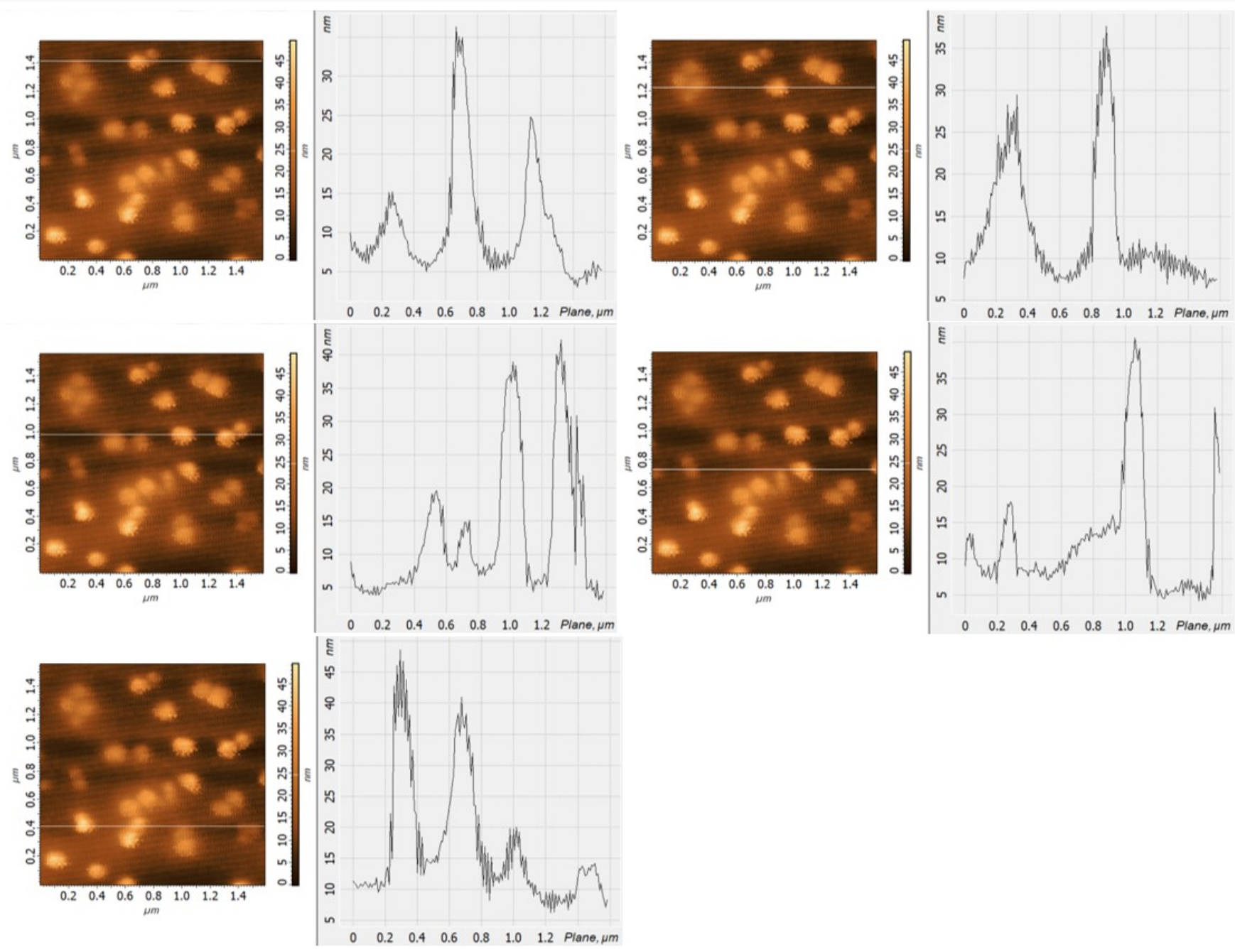

Figure 6.30: Cross sections used to determine the background height in Region A for AFM images taken 60s after laser exposure. These five cross section measurements were replicated in Region A for the AFM images taken before laser exposure and after each of the laser exposure trials. The lowest height in each cross section was considered the lowest value in the cross section with minimal noise.

Table 6.1: Background Height in Region A of AFM Images Before and After Laser Exposure Trials

\begin{tabular}{|c|c|c|c|c|c|c|}
\hline & \multicolumn{6}{|c|}{ Height of Background in Cross Section (nm) } \\
\hline Trial & $\begin{array}{c}\text { Cross } \\
\text { Section 1 }\end{array}$ & $\begin{array}{c}\text { Cross } \\
\text { Section 2 }\end{array}$ & $\begin{array}{c}\text { Cross } \\
\text { Section 3 }\end{array}$ & $\begin{array}{c}\text { Cross } \\
\text { Section 4 }\end{array}$ & $\begin{array}{c}\text { Cross } \\
\text { Section 5 }\end{array}$ & Average ( \pm SD) \\
\hline Before & 12.0 & 14.0 & 8.0 & 10.0 & 10.0 & $10.8 \pm 2.0$ \\
\hline $30 \mathrm{~s}$ & 3.0 & 6.5 & 2.5 & 4.5 & 8.0 & $4.9 \pm 2.1$ \\
\hline $60 \mathrm{~s}$ & 3.0 & 6.5 & 3.0 & 5.0 & 7.5 & $5.0 \pm 1.8$ \\
\hline $90 \mathrm{~s}$ & 3.0 & 7.0 & 2.5 & 5.5 & 5.0 & $4.6 \pm 1.7$ \\
\hline
\end{tabular}




\begin{tabular}{|c|c|c|c|c|c|c|}
\hline $120 \mathrm{~s}$ & 5.0 & 7.5 & 5.0 & 7.0 & 3.5 & $5.6 \pm 1.5$ \\
\hline $180 \mathrm{~s}$ & 32.3 & 35.0 & 32.0 & 34.3 & 35.0 & $33.7 \pm 1.3$ \\
\hline
\end{tabular}
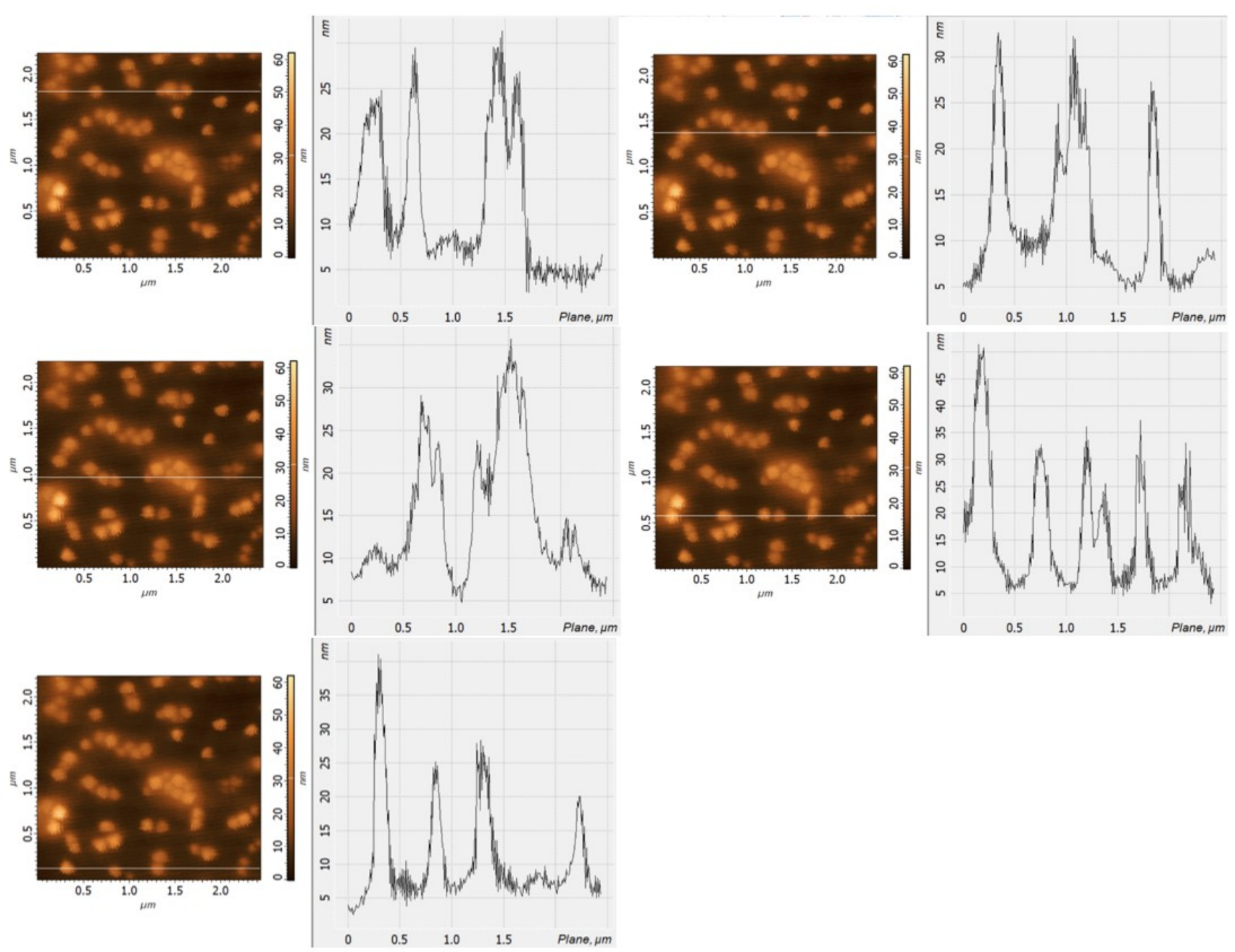

Figure 6.31: Cross sections used to determine the background height in Region B for AFM images taken 60s after laser exposure. These five cross section measurements were replicated in Region B for the AFM images taken before laser exposure and after each of the laser exposure trials. The lowest height in each cross section was considered the lowest value in the cross section with minimal noise.

Table 6.2: Background Height in Region B of AFM Images Before and After Laser Exposure Trials

\begin{tabular}{|c|c|c|c|c|c|c|}
\hline & \multicolumn{6}{|c|}{ Height of Background in Cross Section (nm) } \\
\hline Trial & $\begin{array}{c}\text { Cross } \\
\text { Section 1 }\end{array}$ & $\begin{array}{c}\text { Cross } \\
\text { Section 2 }\end{array}$ & $\begin{array}{c}\text { Cross } \\
\text { Section 3 }\end{array}$ & $\begin{array}{c}\text { Cross } \\
\text { Section 4 }\end{array}$ & $\begin{array}{c}\text { Cross } \\
\text { Section 5 }\end{array}$ & Average ( \pm SD) \\
\hline Before & 7.5 & 6.5 & 11.0 & 6.0 & 11.0 & $8.4 \pm 2.2$ \\
\hline $30 \mathrm{~s}$ & 3.0 & 3.0 & 5.0 & 5.0 & 5.0 & $4.2 \pm 1.0$ \\
\hline $60 \mathrm{~s}$ & 3.5 & 5.0 & 5.0 & 6.0 & 3.0 & $4.5 \pm 1.1$ \\
\hline $90 \mathrm{~s}$ & 3.0 & 2.0 & 6.0 & 3.5 & 5.0 & $3.9 \pm 1.4$ \\
\hline $120 \mathrm{~s}$ & 3.0 & 3.0 & 5.0 & 5.0 & 3.0 & $3.8 \pm 1.0$ \\
\hline $180 \mathrm{~s}$ & 12.5 & 12.0 & 11.5 & 11.5 & 12.0 & $11.9 \pm 0.4$ \\
\hline
\end{tabular}



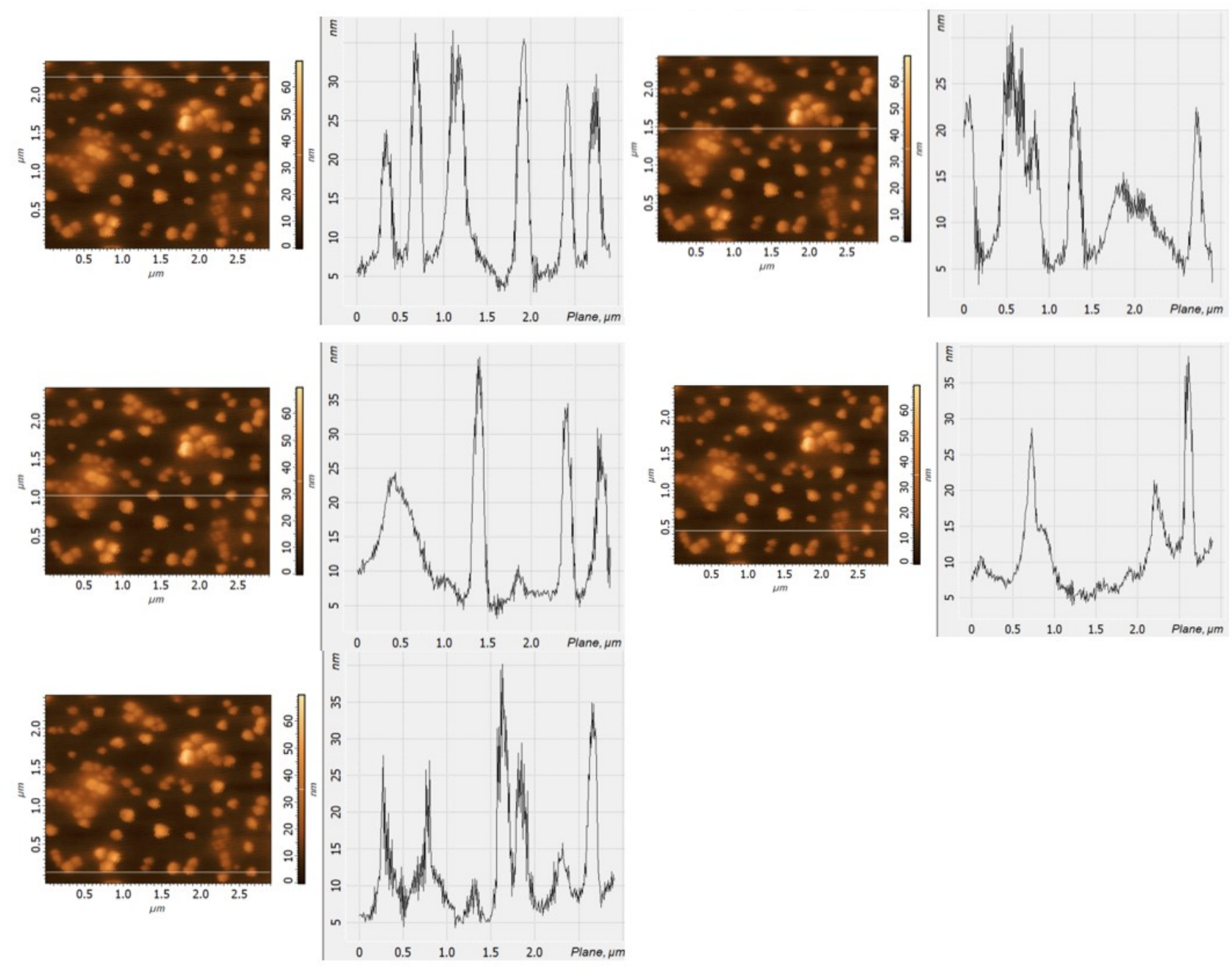

Figure 6.32: Cross sections used to determine the background height in Region C for AFM images taken 60s after laser exposure. These five cross section measurements were replicated in Region C for the AFM images taken before laser exposure and after each of the laser exposure trials. The lowest height in each cross section was considered the lowest value in the cross section with minimal noise.

Table 6.3: Background Height in Region C of AFM Images Before and After Laser Exposure Trials

\begin{tabular}{|c|c|c|c|c|c|c|}
\hline & \multicolumn{6}{|c|}{ Height of Background in Cross Section (nm) } \\
\hline Trial & $\begin{array}{c}\text { Cross } \\
\text { Section 1 }\end{array}$ & $\begin{array}{c}\text { Cross } \\
\text { Section 2 }\end{array}$ & $\begin{array}{c}\text { Cross } \\
\text { Section 3 }\end{array}$ & $\begin{array}{c}\text { Cross } \\
\text { Section 4 }\end{array}$ & $\begin{array}{c}\text { Cross } \\
\text { Section 5 }\end{array}$ & Average ( \pm SD) \\
\hline Before & 8.0 & 8.0 & 10.0 & 20.0 & 13.0 & $11.8 \pm 4.5$ \\
\hline $30 \mathrm{~s}$ & 3.0 & 5.0 & 4.0 & 5.0 & 5.0 & $4.4 \pm 0.8$ \\
\hline $60 \mathrm{~s}$ & 4.0 & 5.0 & 5.0 & 5.0 & 5.0 & $4.8 \pm 0.4$ \\
\hline $90 \mathrm{~s}$ & 6.5 & 6.5 & 6.5 & 10.0 & 8.0 & $7.5 \pm 1.4$ \\
\hline $120 \mathrm{~s}$ & 5.0 & 5.5 & 5.0 & 6.0 & 6.5 & $5.6 \pm 0.6$ \\
\hline $180 \mathrm{~s}$ & 3.0 & 5.5 & 5.5 & 5.5 & 7.0 & $5.3 \pm 1.3$ \\
\hline
\end{tabular}


6.4.3 Height Measurement of AgNCs in Regions A, B and C

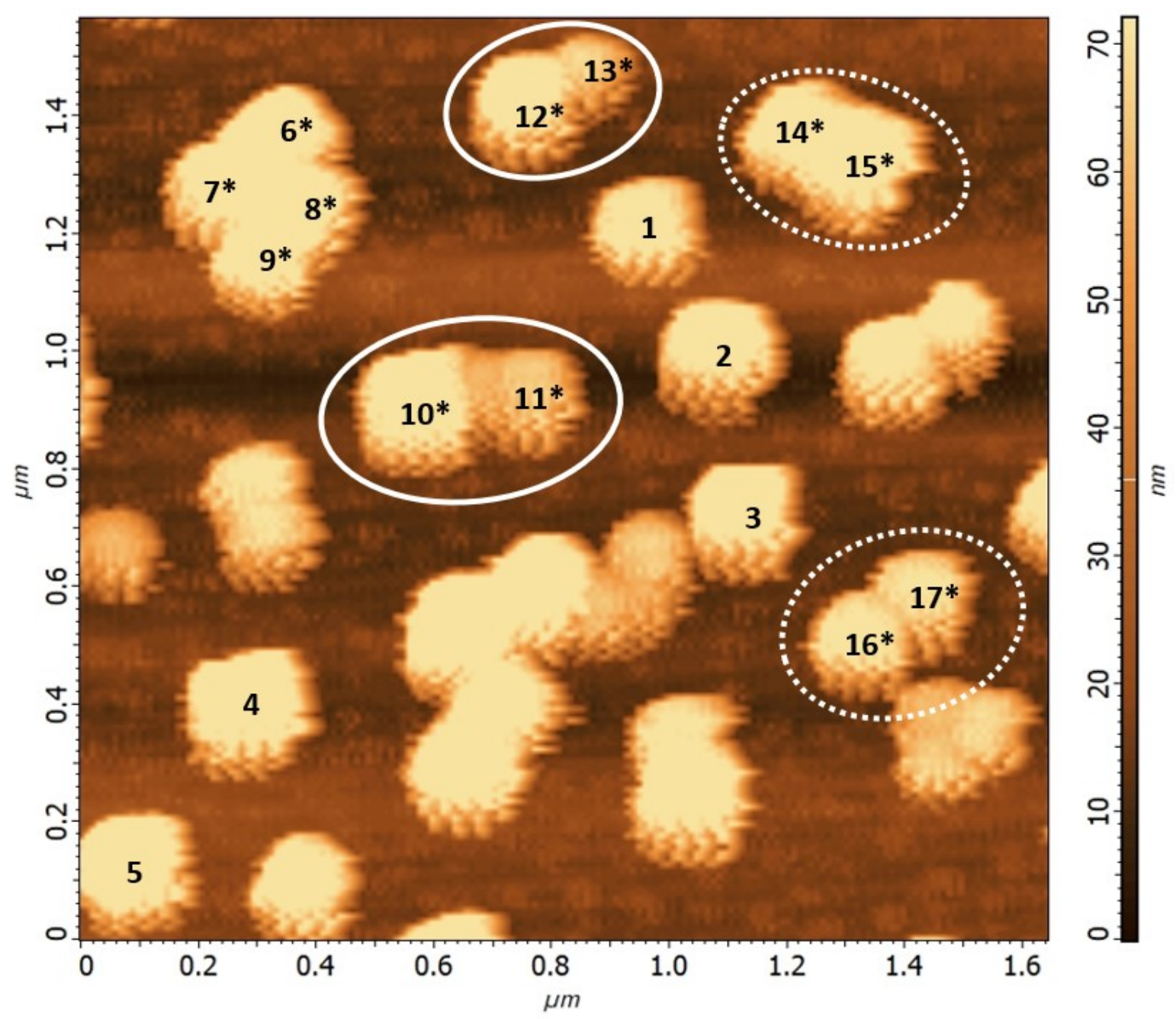

Figure 6.33: Close up of Region A used for cube height measurements. The cubes whose heights were measured are numbered. An asterix next to the number indicates the cubes that are considered to be in clusters. DS Dimers and SS Dimers are circled and identified by solid and dotted lines respectively. The numbering is shown here on the AFM taken before laser exposure for reference.

Table 6.4: Corrected Height of AgNCs Before Laser Exposure and After Laser Exposure Intervals in Region A

\begin{tabular}{|c|c|c|c|c|c|c|c|}
\hline \multicolumn{2}{|c|}{} & \multicolumn{5}{|c|}{ Height of Cube After Different Intervals of Laser Exposure (nm) } \\
\hline $\begin{array}{c}\text { Type of } \\
\text { Cube }\end{array}$ & Number & Before & After 30s & After 60s & After 90s & After 120s & After 180s \\
\hline Individual & 1 & 61.1 & 31.8 & 30.7 & 30.4 & 25.5 & 30.4 \\
\cline { 2 - 8 } & 2 & 61.1 & 39.6 & 30.9 & 35.1 & 31.0 & 35.3 \\
\cline { 2 - 8 } & 3 & 61.1 & 29.5 & 27.6 & 33.9 & 32.0 & 34.2 \\
\cline { 2 - 8 } & 4 & 61.1 & 39.2 & 39.4 & 39.3 & 42.1 & 43.1 \\
\cline { 2 - 8 } & 5 & 61.1 & 40.6 & 42.9 & 39.9 & 38.8 & 38.4 \\
\hline & 6 & 61.1 & 24.5 & 18.0 & 16.2 & 16.1 & 15.6 \\
\hline
\end{tabular}




\begin{tabular}{|c|c|c|c|c|c|c|c|}
\hline \multirow{3}{*}{$\begin{array}{c}\text { In } \\
\text { Diamond }\end{array}$} & 7 & 61.1 & 25.1 & 23.5 & 21.9 & 14.6 & 14.3 \\
\cline { 2 - 7 } Tetramer & 8 & 61.1 & 28.2 & 24.1 & 20.1 & 18.8 & 18.3 \\
\cline { 2 - 7 } In DS & 9 & 61.1 & 24.8 & 20.2 & 17.3 & 16.6 & 15.4 \\
\cline { 2 - 7 } Dimer & 10 & 61.1 & 24.1 & 23.5 & 23.0 & 20.9 & 18.1 \\
\cline { 2 - 7 } & 11 & 56.9 & 19.0 & 20.8 & 17.5 & 16.6 & 12.4 \\
\cline { 2 - 8 } & 12 & 61.1 & 32.1 & 20.6 & 27.0 & 25.5 & 28.0 \\
\hline \multirow{3}{*}{ In SS } & 13 & 60.4 & 22.2 & 17.8 & 16.0 & 13.7 & 18.0 \\
\cline { 2 - 8 } Dimer & 14 & 61.1 & 26.2 & 21.5 & 22.6 & 22.9 & 20.5 \\
\cline { 2 - 8 } & 15 & 61.1 & 31.1 & 29.1 & 26.3 & 27.0 & 26.7 \\
\cline { 2 - 8 } & 16 & 61.1 & 21.9 & 23.4 & 21.5 & 19.8 & 19.1 \\
\hline
\end{tabular}

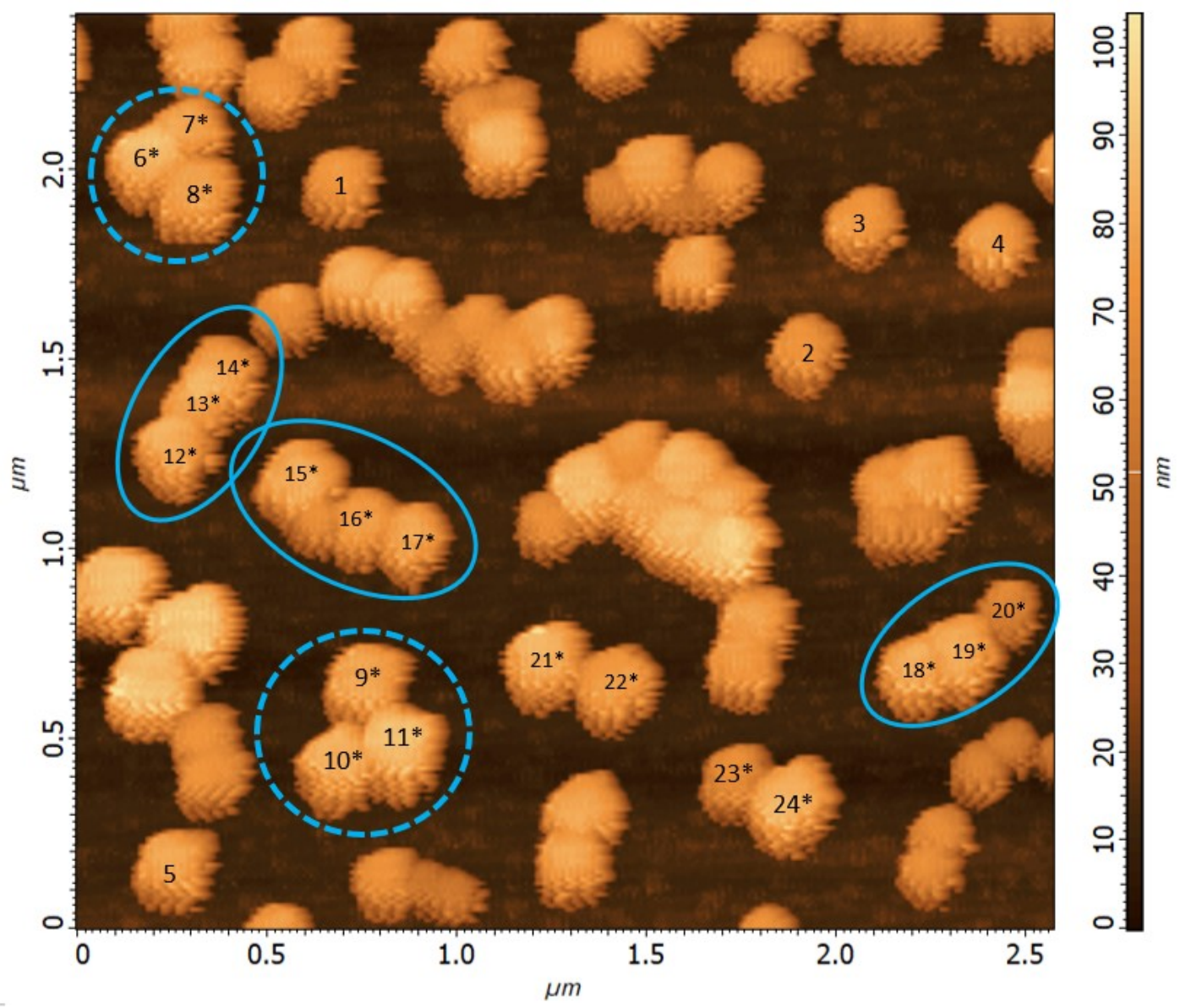

Figure 6.34: Close up of Region B used for cube height measurements. The cubes whose heights were measured are numbered. An asterix next to the number indicates the cubes that are considered to be in clusters. Row Trimers and Triangular Trimers are circled and identified by solid and dotted lines respectively. Cubes 21 and 22 are considered to be in a SS Dimer and cubes 23 and 24 are considered to be in a DS Dimer. The numbering is shown here on the AFM taken before laser exposure for reference.

Table 6.5: Corrected Height of AgNCs Before Laser Exposure and After Laser Exposure Intervals in Region B 


\begin{tabular}{|c|c|c|c|c|c|c|c|}
\hline $\begin{array}{l}\text { Type of } \\
\text { Cube }\end{array}$ & Number & Before & After 30s & After $60 \mathrm{~s}$ & After 90s & After $120 \mathrm{~s}$ & After $180 \mathrm{~s}$ \\
\hline \multirow{5}{*}{$\begin{array}{c}\text { Individual } \\
\text { Cubes }\end{array}$} & 1 & 66.9 & 29.3 & 24.9 & 21.2 & 21.7 & 24.0 \\
\hline & 2 & 67.8 & 25.8 & 21.6 & 14.8 & 20.8 & 19.5 \\
\hline & 3 & 69.3 & 32.2 & 26.0 & 22.2 & 22.3 & 21.6 \\
\hline & 4 & 70.0 & 26.4 & 31.5 & 28.7 & 26.8 & 30.6 \\
\hline & 5 & 71.2 & 34.9 & 33.5 & 30.7 & 30.4 & 31.2 \\
\hline \multirow{6}{*}{$\begin{array}{c}\text { In Triangle } \\
\text { Trimers }\end{array}$} & 6 & 76.8 & 22.4 & 20.1 & 23.7 & 19.9 & 17.3 \\
\hline & 7 & 70.0 & 22.5 & 19.7 & 21.4 & 19.9 & 18.4 \\
\hline & 8 & 71.2 & 19.2 & 14.9 & 17.9 & 15.9 & 16.0 \\
\hline & 9 & 71.7 & 23.9 & 25.7 & 23.9 & 21.1 & 18.3 \\
\hline & 10 & 73.7 & 34.6 & 34.8 & 32.1 & 30.8 & 31.1 \\
\hline & 11 & 80.1 & 39.2 & 26.5 & 34.9 & 34.8 & 33.1 \\
\hline \multirow{9}{*}{$\begin{array}{l}\text { In Row } \\
\text { Trimers }\end{array}$} & 12 & 73.7 & 27.5 & 26.5 & 24.1 & 24.0 & 25.2 \\
\hline & 13 & 70.7 & 15.9 & 17.6 & 18.0 & 15.8 & 19.6 \\
\hline & 14 & 72.2 & 27.7 & 26.2 & 25.0 & 28.0 & 27.1 \\
\hline & 15 & 75.6 & 25.1 & 25.2 & 24.6 & 24.1 & 25.3 \\
\hline & 16 & 69.8 & 22.9 & 21.8 & 23.5 & 22.7 & 23.3 \\
\hline & 17 & 74.5 & 19.7 & 16.6 & 21.0 & 16.0 & 22.6 \\
\hline & 18 & 77.4 & 29.9 & 26.6 & 24.1 & 26.7 & 22.1 \\
\hline & 19 & 75.6 & 35.9 & 33.0 & 31.6 & 29.6 & 31.8 \\
\hline & 20 & 54.9 & 19.6 & 17.6 & 19.6 & 15.9 & 17.0 \\
\hline \multirow{2}{*}{$\begin{array}{l}\text { In SS } \\
\text { Dimer }\end{array}$} & 21 & 78.2 & 35.1 & 31.2 & 27.9 & 29.6 & 29.0 \\
\hline & 22 & 70.3 & 27.2 & 19.5 & 13.6 & 16.4 & 16.6 \\
\hline \multirow{2}{*}{$\begin{array}{l}\text { In DS } \\
\text { Dimer }\end{array}$} & 23 & 57.3 & 22.1 & 18.5 & 17.2 & 16.6 & 15.6 \\
\hline & 24 & 81.3 & 32.5 & 28.0 & 26.5 & 22.6 & 24.7 \\
\hline
\end{tabular}



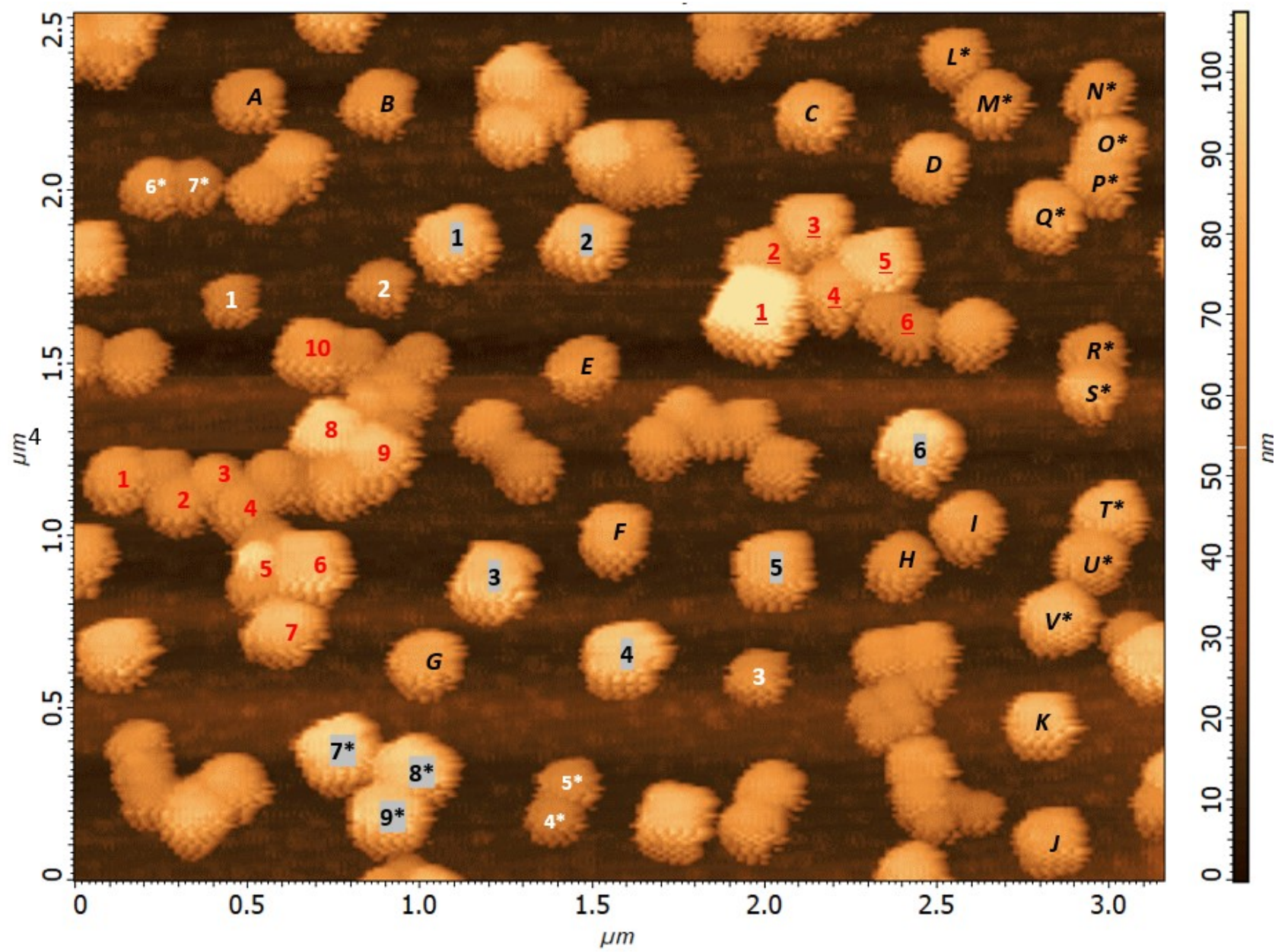

Figure 6.35: Close up of Region C used for cube height measurements. The cubes whose heights were measured are numbered and lettered. In region $C$ the cubes are classified by size: the cubes with white numbers, black letters, and black numbers with grey highlight and are classified as small, medium and large respectively. For these cubes an asterix indicates that they are in a small cluster (4 cubes or less). The cubes with red numbers are cubes in clusters with $5+$ cubes. The nonunderlined red numbers indicate cubes in large cluster I and the underlined red numbers indicate cubes in large cluster II. The numbering is shown here on the AFM taken before laser exposure for reference.

\section{Table 6.6: Corrected Height of AgNCs Before Laser Exposure and After Laser Exposure Intervals for Small Cubes in Region C}

\begin{tabular}{|c|c|c|c|c|c|c|c|}
\hline \multicolumn{2}{|c|}{} & \multicolumn{5}{|c|}{ Height of Cube After Different Intervals of Laser Exposure (nm) } \\
\hline $\begin{array}{c}\text { Type of } \\
\text { Cube }\end{array}$ & Number & Before & After 30s & After 60s & After 90s & After 120s & After 180s \\
\hline \multirow{2}{*}{$\begin{array}{c}\text { Individual } \\
\text { Cube }\end{array}$} & 1 & 51.5 & 16.0 & 14.4 & 15.4 & 12.3 & 19.9 \\
\cline { 2 - 8 } & 2 & 51.5 & 20.7 & 20.3 & 19.8 & 18.5 & 18.4 \\
\cline { 2 - 8 } & 3 & 56.0 & 20.9 & 22.8 & 21.7 & 17.3 & 21.0 \\
\hline \multirow{2}{*}{$\begin{array}{c}\text { In DS } \\
\text { Dimers }\end{array}$} & 4 & 46.7 & 12.0 & 8.9 & 12.5 & 14.0 & 11.2 \\
\cline { 2 - 8 } & 5 & 54.7 & 14.6 & 16.2 & 16.0 & 15.5 & 15.8 \\
\cline { 2 - 8 } & 7 & 55.8 & 16.7 & 18.8 & 18.5 & 16.6 & 17.2 \\
\cline { 2 - 8 } & 51.9 & 16.2 & 16.6 & 18.0 & 11.9 & 19.4 \\
\hline
\end{tabular}


Table 6.7: Corrected Height of AgNCs Before Laser Exposure and After Laser Exposure Intervals for Medium Cubes in Region C

\begin{tabular}{|c|c|c|c|c|c|c|c|}
\hline \multirow[b]{2}{*}{$\begin{array}{l}\text { Type of } \\
\text { Cube }\end{array}$} & \multirow[b]{2}{*}{ Letter } & \multicolumn{6}{|c|}{ Height of Cube After Different Intervals of Laser Exposure (nm) } \\
\hline & & Before & After 30s & After 60s & After 90s & After 120s & After $180 \mathrm{~s}$ \\
\hline \multirow[t]{11}{*}{ Individual } & A & 59.1 & 15.4 & 14.3 & 19.1 & 17.9 & 18.4 \\
\hline & $\mathrm{B}$ & 64.9 & 27.9 & 27.6 & 29.4 & 28.7 & 26.6 \\
\hline & C & 69.1 & 33.8 & 31.8 & 28.7 & 31.6 & 29.7 \\
\hline & $\mathrm{D}$ & 70.1 & 33.4 & 32.5 & 29.3 & 32.8 & 32.6 \\
\hline & $E$ & 67.4 & 21.9 & 18.2 & 18.3 & 17.7 & 20.3 \\
\hline & $\mathrm{F}$ & 70.1 & 34.7 & 32.0 & 32.7 & 38.0 & 36.3 \\
\hline & G & 65.2 & 31.8 & 25.5 & 25.0 & 22.4 & 27.4 \\
\hline & $\mathrm{H}$ & 60.6 & 21.6 & 22.5 & 15.0 & 24.7 & 24.4 \\
\hline & 1 & 63.7 & 29.0 & 23.3 & 28.4 & 28.0 & 29.0 \\
\hline & $\mathrm{J}$ & 69.8 & 23.7 & 30.1 & 27.8 & 25.5 & 27.6 \\
\hline & K & 72.9 & 30.6 & 33.1 & 31.7 & 31.7 & 31.3 \\
\hline \multirow{2}{*}{$\begin{array}{l}\text { In SS } \\
\text { Dimer }\end{array}$} & $L$ & 63.4 & 26.5 & 25.5 & 24.1 & 21.1 & 21.9 \\
\hline & $M$ & 61.6 & 23.4 & 20.7 & 27.4 & 25.4 & 23.7 \\
\hline \multirow{4}{*}{$\begin{array}{l}\text { In Row } \\
\text { Tetramer }\end{array}$} & $\mathrm{N}$ & 76.9 & 34.1 & 33.4 & 32.4 & 35.1 & 33.8 \\
\hline & 0 & 62.5 & 30.2 & 26.5 & 31.4 & 31.2 & 33.8 \\
\hline & $P$ & 74.6 & 37.0 & 36.7 & 33.9 & 34.9 & 38.2 \\
\hline & $Q$ & 60.1 & 19.9 & 24.5 & 16.8 & 22.2 & 21.6 \\
\hline \multirow{2}{*}{$\begin{array}{l}\text { In SS } \\
\text { Dimer }\end{array}$} & $R$ & 60.8 & 21.6 & 19.6 & 21.1 & 25.5 & 22.6 \\
\hline & $S$ & 75.4 & 21.7 & 21.0 & 26.7 & 28.1 & 25.3 \\
\hline \multirow{3}{*}{$\begin{array}{l}\text { In Row } \\
\text { Trimer }\end{array}$} & $T$ & 73.1 & 23.4 & 22.4 & 24.1 & 24.5 & 26.3 \\
\hline & $U$ & 68.6 & 25.7 & 27.5 & 24.9 & 26.7 & 27.4 \\
\hline & $\mathrm{V}$ & 80.9 & 29.8 & 29.3 & 29.1 & 32.6 & 31.0 \\
\hline
\end{tabular}

Table 6.8: Corrected Height of AgNCs Before Laser Exposure and After Laser Exposure Intervals for Large Cubes in Region $C$

\begin{tabular}{|c|c|c|c|c|c|c|c|}
\hline \multicolumn{2}{|c|}{} & \multicolumn{5}{|c|}{ Height of Cube After Different Intervals of Laser Exposure (nm) } \\
\hline $\begin{array}{c}\text { Type of } \\
\text { Cube }\end{array}$ & Number & Before & After 30s & After 60s & After 90s & After 120s & After 180s \\
\hline \multirow{4}{*}{$\begin{array}{c}\text { Individual } \\
\text { Cube }\end{array}$} & 1 & 76.9 & 37.8 & 35.9 & 27.0 & 27.5 & 30.2 \\
\cline { 2 - 8 } & 2 & 72.7 & 41.6 & 35.6 & 36.1 & 37.3 & 42.6 \\
\cline { 2 - 8 } & 3 & 77.9 & 34.5 & 37.8 & 38.9 & 38.3 & 35.0 \\
\cline { 2 - 8 } & 4 & 79.4 & 45.0 & 43.4 & 39.3 & 40.9 & 45.0 \\
\cline { 2 - 8 } & 5 & 74.9 & 38.7 & 35.2 & 31.6 & 34.6 & 37.3 \\
\cline { 2 - 8 } & 6 & 75.9 & 42.9 & 36.3 & 39.0 & 38.7 & 35.9 \\
\hline \multirow{4}{*}{$\begin{array}{c}\text { In Triangle } \\
\text { Trimer }\end{array}$} & 7 & 89.6 & 38.3 & 36.5 & 40.7 & 39.3 & 34.2 \\
\cline { 2 - 8 } & 8 & 82.7 & 51.3 & 48.7 & 41.5 & 49.3 & 45.1 \\
\cline { 2 - 8 } & 9 & 83.6 & 41.6 & 37.4 & 35.3 & 41.4 & 42.8 \\
\hline
\end{tabular}


Table 6.9: Corrected Height of AgNCs Before Laser Exposure and After Laser Exposure Intervals for Cubes in Large Clusters Region $C$

\begin{tabular}{|c|c|c|c|c|c|c|c|}
\hline \multicolumn{2}{|c|}{} & \multicolumn{5}{|c|}{ Height of Cube After Different Intervals of Laser Exposure (nm) } \\
\hline $\begin{array}{c}\text { Type of } \\
\text { Cube }\end{array}$ & Number & Before & After 30s & After 60s & After 90s & After 120s & After 180s \\
\hline $\begin{array}{c}\text { In Large } \\
\text { Cluster I }\end{array}$ & 1 & 63.0 & 19.9 & 25.3 & 22.1 & 21.1 & 20.6 \\
\cline { 2 - 8 } & 2 & 59.7 & 23.0 & 17.3 & 15.9 & 16.3 & 15.6 \\
\cline { 2 - 8 } & 3 & 63.0 & 26.8 & 22.5 & 15.1 & 22.3 & 14.7 \\
\cline { 2 - 8 } & 4 & 58.6 & 21.6 & 26.1 & 23.5 & 23.4 & 24.6 \\
\cline { 2 - 8 } & 5 & 77.5 & 32.5 & 24.3 & 21.6 & 20.4 & 26.2 \\
\cline { 2 - 8 } & 6 & 80.1 & 25.3 & 29.5 & 23.0 & 23.3 & 24.8 \\
\cline { 2 - 8 } & 7 & 75.8 & 31.6 & 29.5 & 33.6 & 31.9 & 27.3 \\
\cline { 2 - 8 } & 8 & 88.7 & 37.4 & 40.9 & 37.1 & 38.3 & 41.6 \\
\cline { 2 - 8 } & 9 & 78.8 & 37.1 & 34.4 & 36.0 & 38.0 & 39.0 \\
\hline \multirow{4}{*}{\begin{tabular}{l} 
In Large \\
\cline { 2 - 8 }
\end{tabular}} & 10 & 62.4 & 20.7 & 20.7 & 20.5 & 22.0 & 22.7 \\
\cline { 2 - 8 } & 2 & 95.4 & 50.8 & 51.6 & 58.6 & 51.4 & 45.9 \\
\cline { 2 - 8 } & 3 & 74.4 & 46.0 & 38.4 & 43.1 & 47.7 & 45.8 \\
\cline { 2 - 8 } & 4 & 78.6 & 27.4 & 30.0 & 31.9 & 33.0 & 25.0 \\
\cline { 2 - 8 } & 5 & 80.2 & 37.9 & 42.6 & 35.7 & 35.4 & 34.4 \\
\cline { 2 - 8 } & 6 & 55.1 & 19.5 & 19.5 & 16.1 & 21.1 & 18.6 \\
\hline
\end{tabular}

Table 6.10: Comparison of Average Change in Height After Initial 30s Laser Exposure ( $\Delta \mathrm{h}_{0-30 \mathrm{~s}}$ ) and after Total 3 min of Exposure ( $\Delta h_{0-180 s}$ ) of Different Types of Cube Clusters in the Three Different Regions

\begin{tabular}{|c|c|c|}
\hline Region & Type of Cube & $\Delta \mathrm{h}_{0-180 \mathrm{~s}}(\mathrm{~nm})$ \\
\hline \multirow{4}{*}{ A } & In Diamond Tetramer & $45.2 \pm 1.7$ \\
\cline { 2 - 3 } & In DS Dimer & $40.8 \pm 4.2$ \\
\cline { 2 - 3 } & In SS Dimer & $40.2 \pm 4.0$ \\
\hline \multirow{4}{*}{ B } & In Row Trimer & $47.8 \pm 5.2$ \\
\cline { 2 - 3 } & In Triangle Trimer & $51.5 \pm 6.0$ \\
\cline { 2 - 3 } & In DS Dimer & $49.1 \pm 10.54$ \\
\cline { 2 - 3 } & In SS Dimer & $49.1 \pm 3.2$ \\
\hline \multirow{4}{*}{ C } & In Row Trimer & $46.0 \pm 4.4$ \\
\cline { 2 - 3 } & In Triangle Timer & $44.6 \pm 9.5$ \\
\cline { 2 - 3 } & In DS Dimer & $35.6 \pm 4.3$ \\
\cline { 2 - 3 } & In SS Dimer & $40.4 \pm 5.5$ \\
\hline
\end{tabular}

6.5 Supplementary Material for Section 3.5 


\subsubsection{FDTD Calculated Absorption Spectra for Different Sizes of AgNC and Different} Shapes and Sized of AgNC Clusters

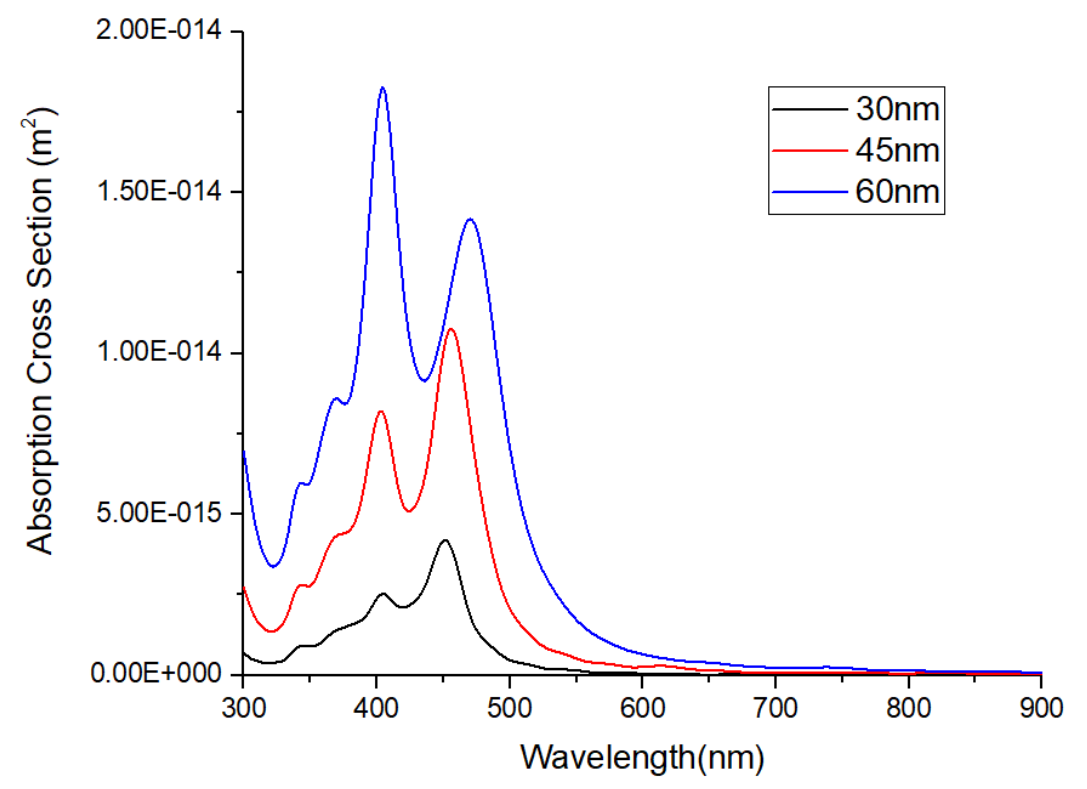

Figure 6.36: FDTD Calculated Absorption Spectra for AgNC of Different sizes.

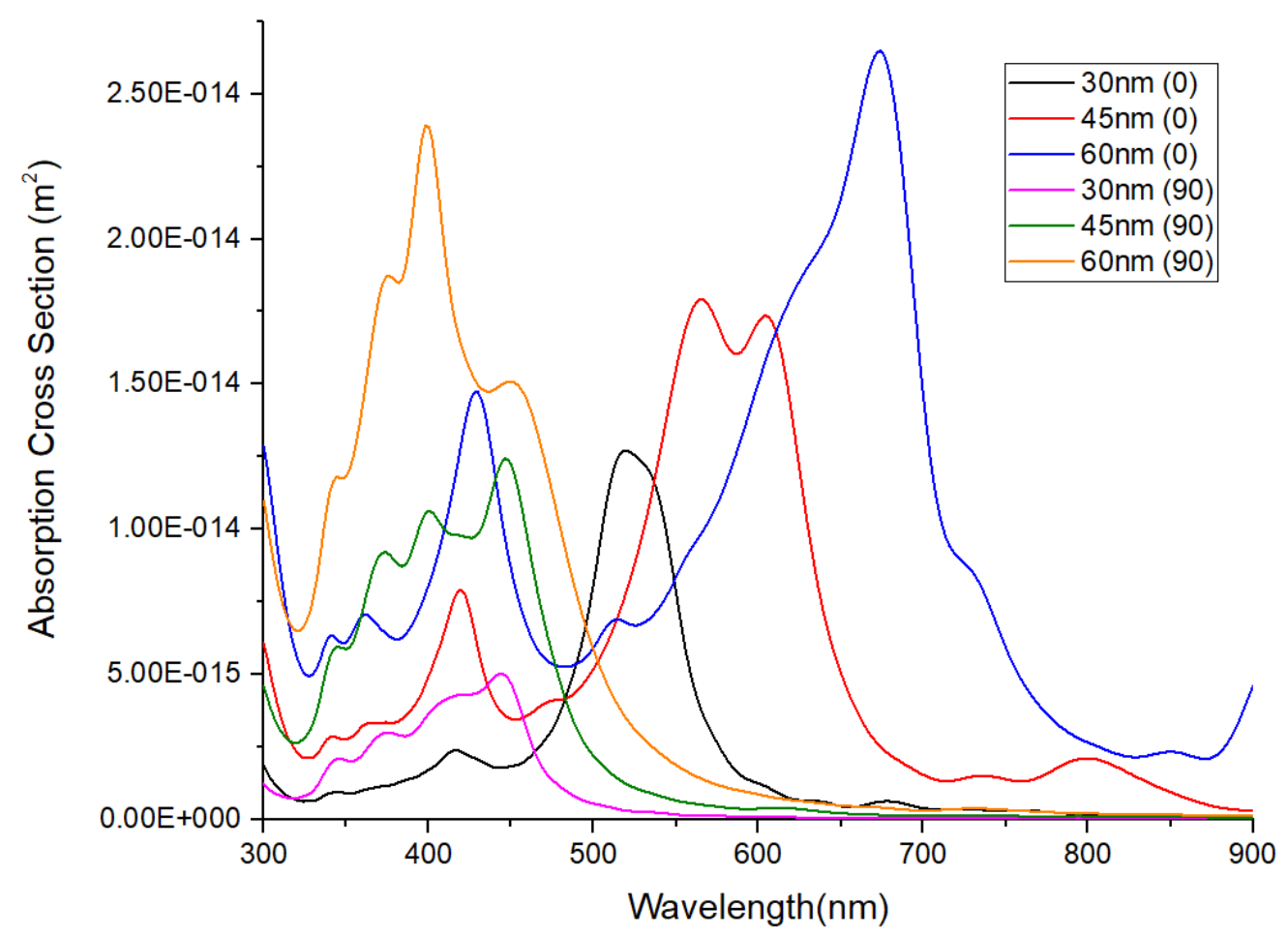

Figure 6.37: FDTD Calculated Absorption Spectra for SS Dimers of AgNC. The numbers in brackets in the legend indicate the angle of polarization. 


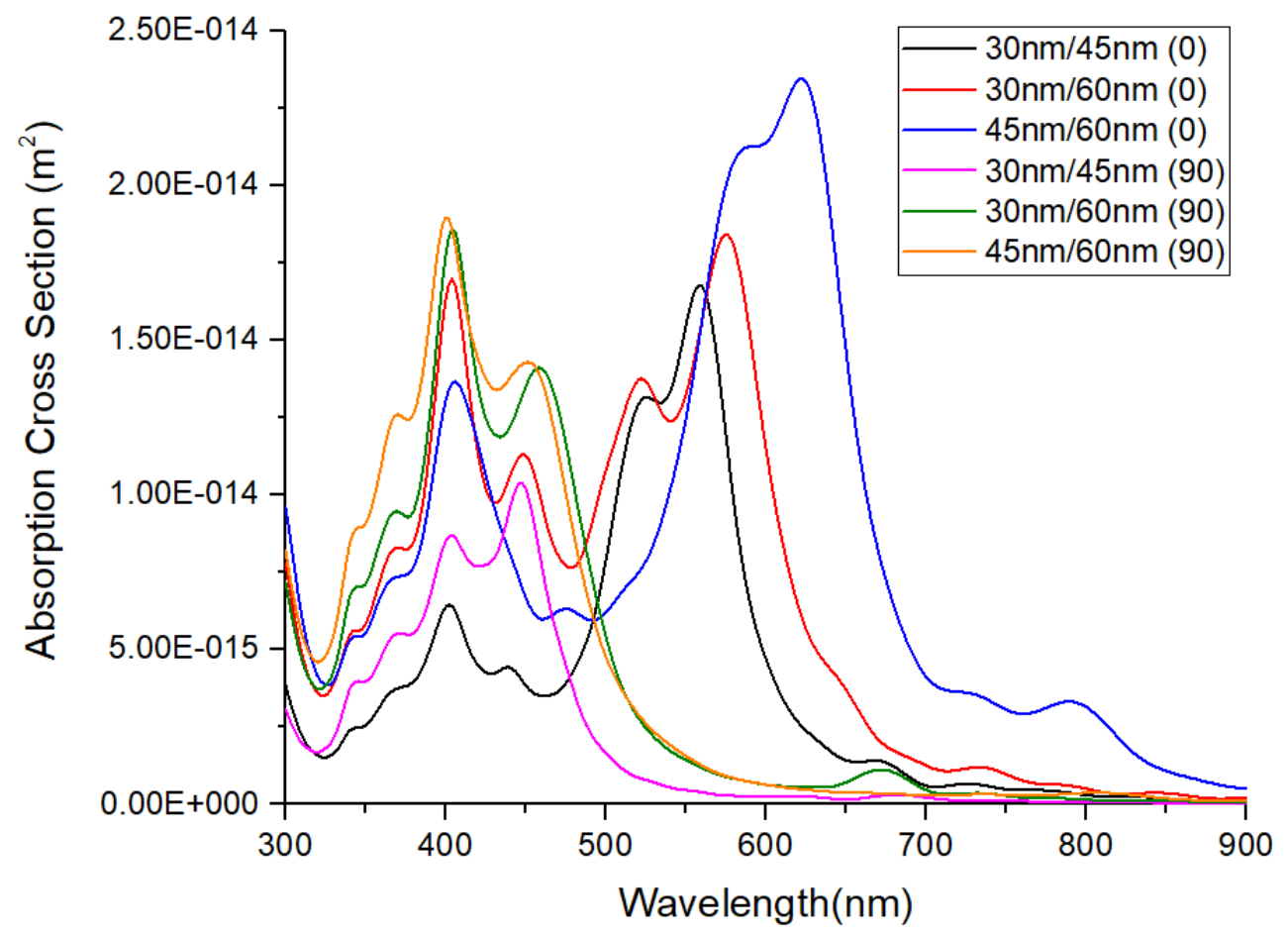

Figure 6.38: FDTD Calculated Absorption Spectra for DS Dimers of AgNC. The numbers in brackets in the legend indicate the angle of polarization.

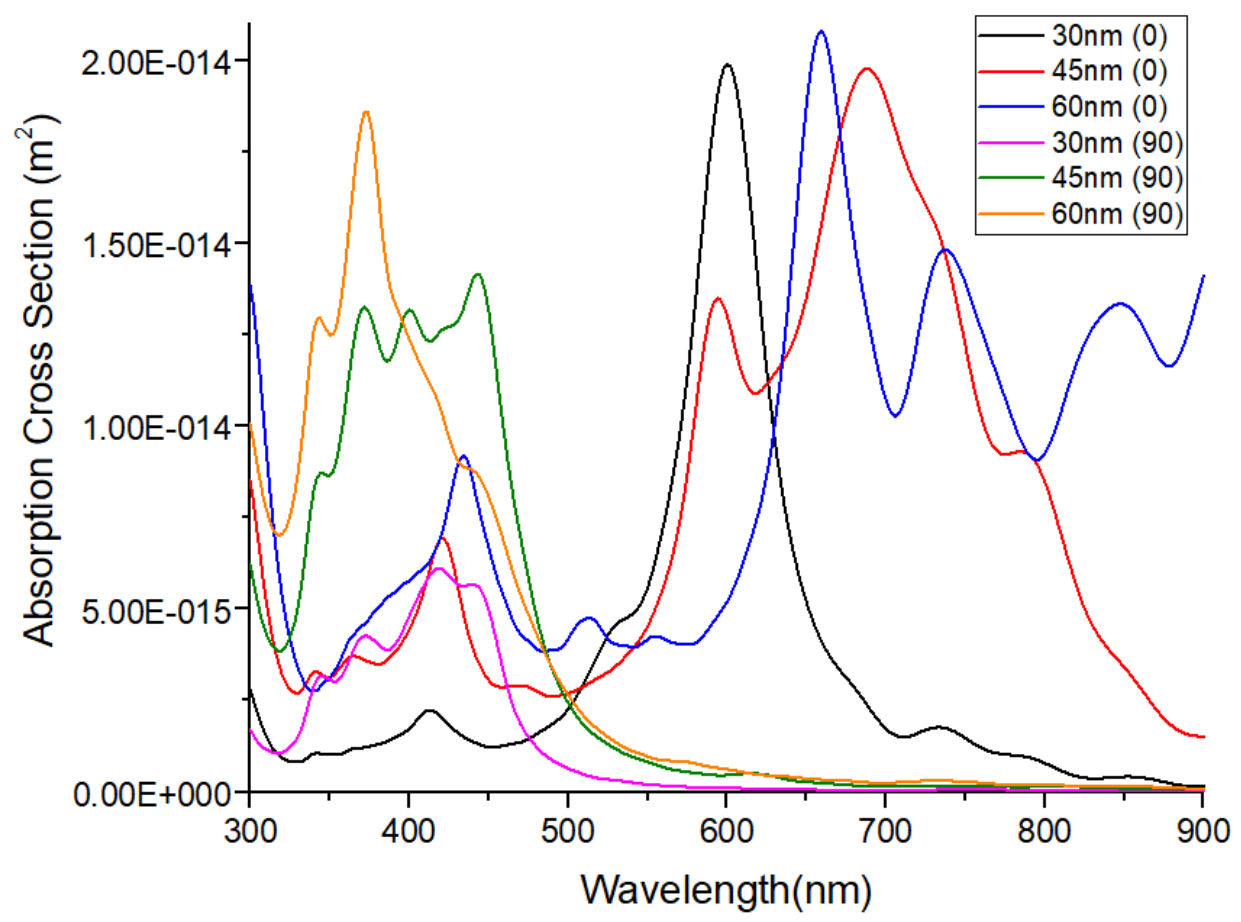

Figure 6.39: FDTD Calculated Absorption Spectra for Row Trimers of AgNC of Different sizes. The numbers in brackets in the legend indicate the angle of polarization. 


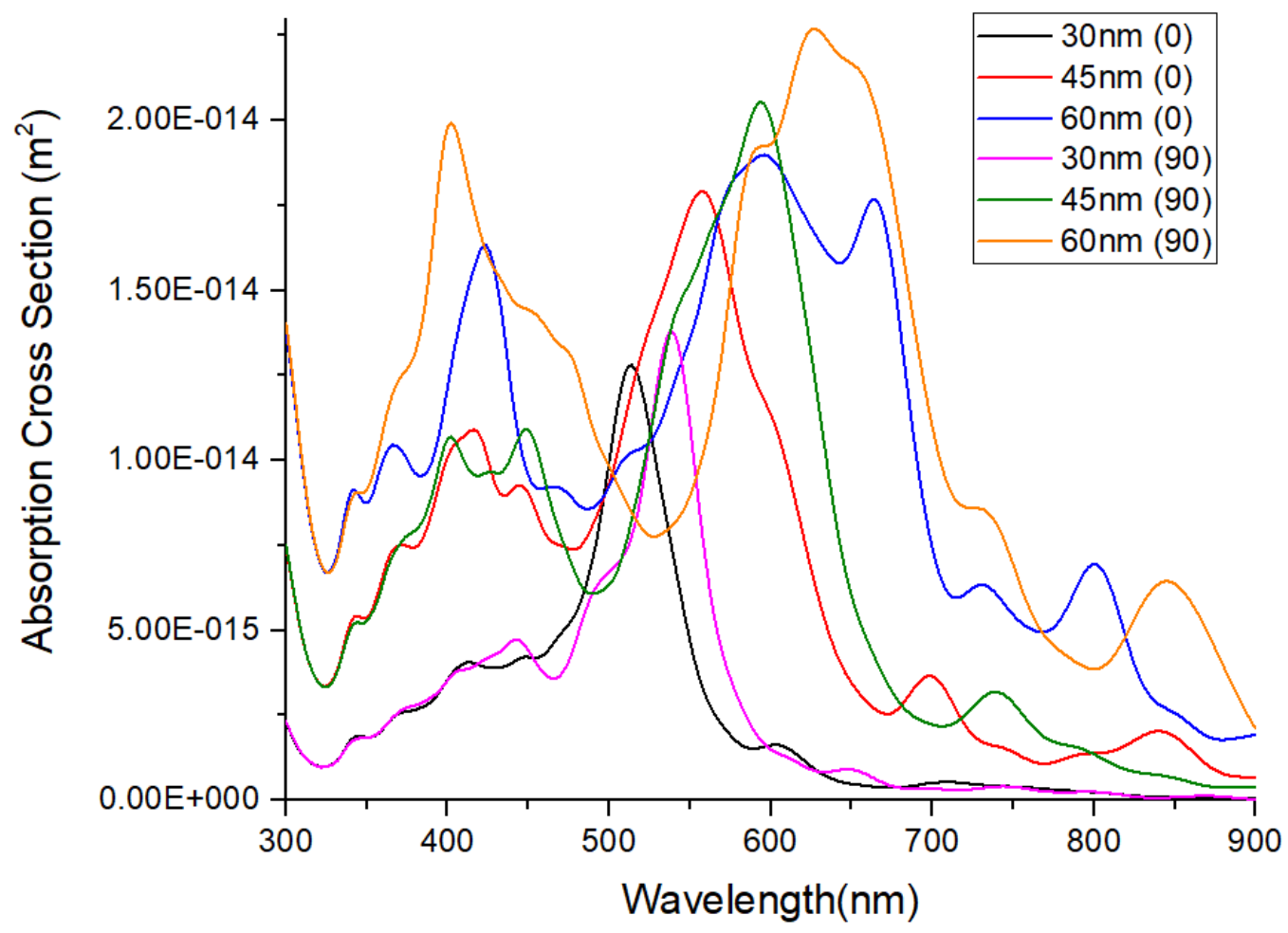

Figure 6.40: FDTD Calculated Absorption Spectra for Triangle Trimers of AgNC of Different sizes. The numbers in brackets in the legend indicate the angle of polarization. 


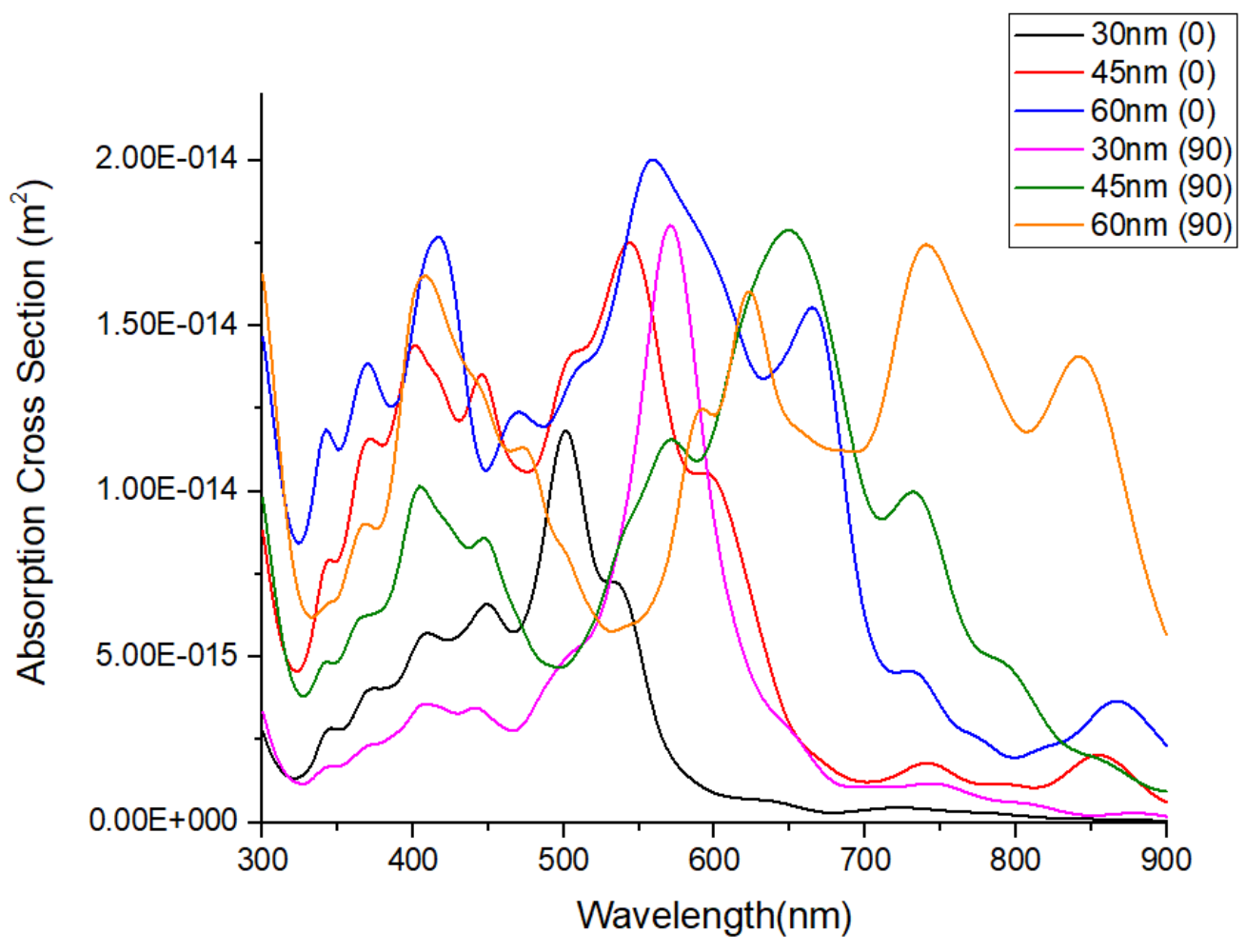

Figure 6.41: FDTD Calculated Absorption Spectra for Diamond Tetramers of AgNC of Different sizes. The numbers in brackets in the legend indicate the angle of polarization.

\subsubsection{Different 2D-Planes Used for the E-Field Enhancement (EEF) Images of AgNC Clusters}
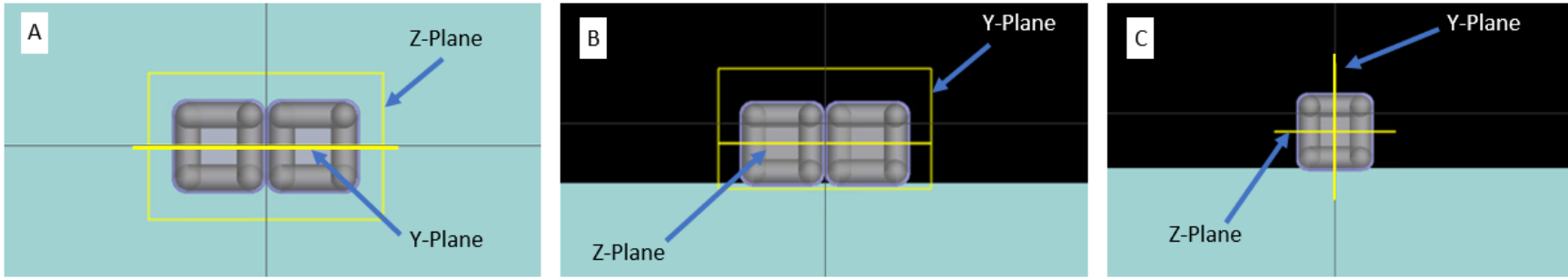

Figure 6.42: 2D plans used to calculate the EEFs for the dimers of AgNCs where nanocubes are the same size. The planes are shown on the $x y(A), x z(B)$ and $y z(C)$ axis. 

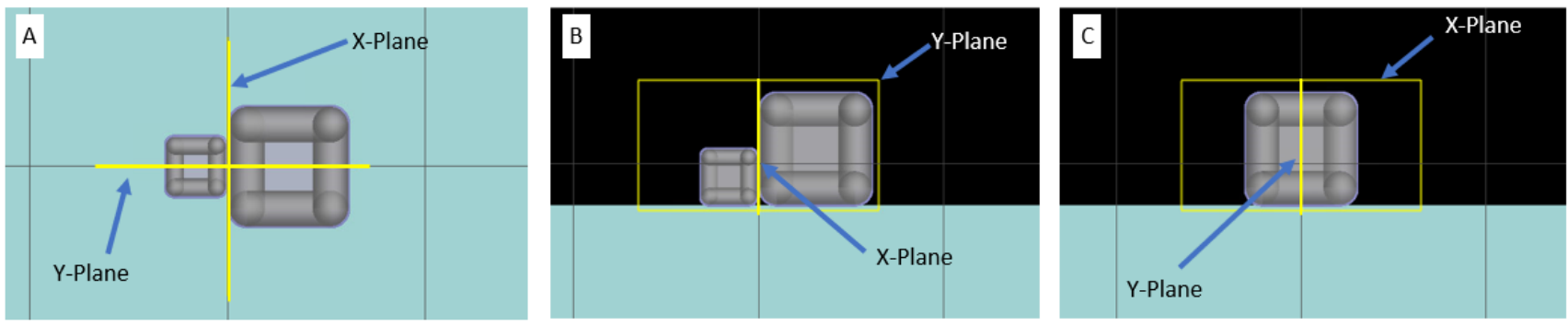

Figure 6.43: 2D planes used to calculate the EEFs for the dimers of AgNCs where nanocubes are the different sizes. The planes are shown on the $x y(A), x z(B)$ and $y z(C)$ axis.
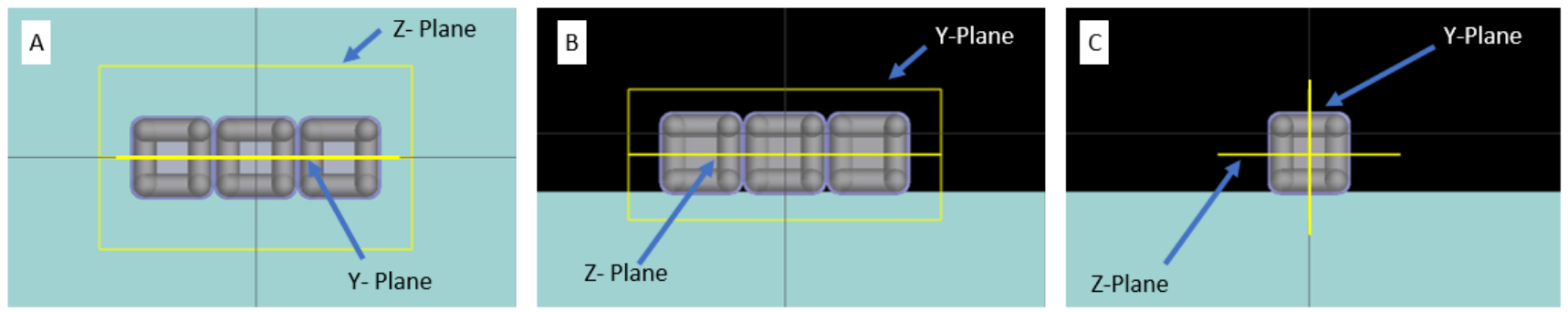

Figure 6.44: 2D planes used to calculate the EEFs for the row trimers of AgNCs. The planes are shown on the $x y(A), x z(B)$ and $y z$ (C) axis.
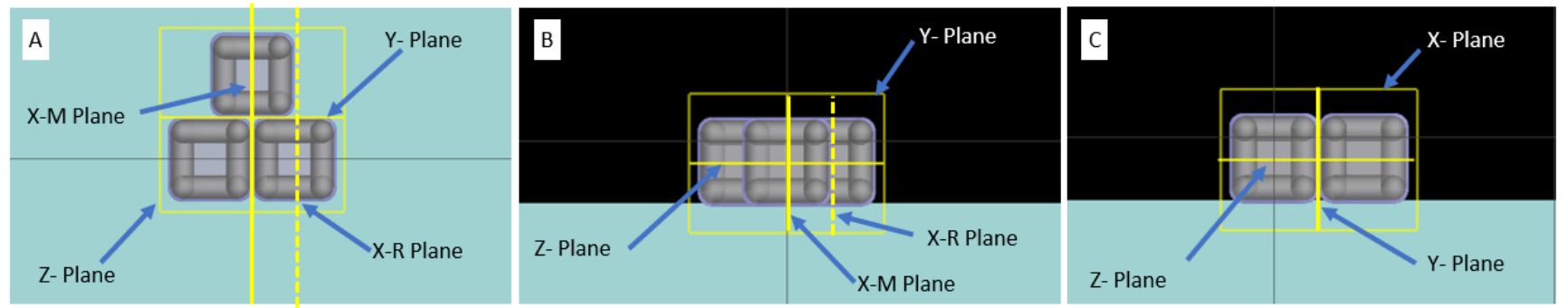

Figure 6.45: 2D planes used to calculate the EEFs for the triangle trimers of AgNCs. The planes are shown on the $x y(A), x z(B)$ and $y z(C)$ axis.
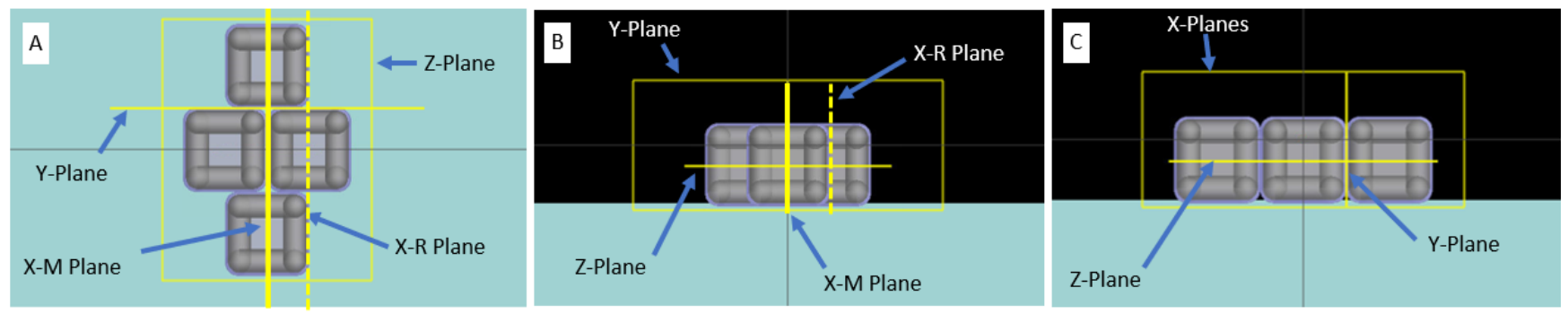

Figure 6.46: 2D planes used to calculate EEF for the diamond tetramers of AgNCs. The planes are shown on the $x y(A), x z(B)$ and $y z(C)$ axis. 
6.5.3 EEF Images for Different Sizes and Shaped of Nanocube Clusters at $568 \mathrm{~nm}$
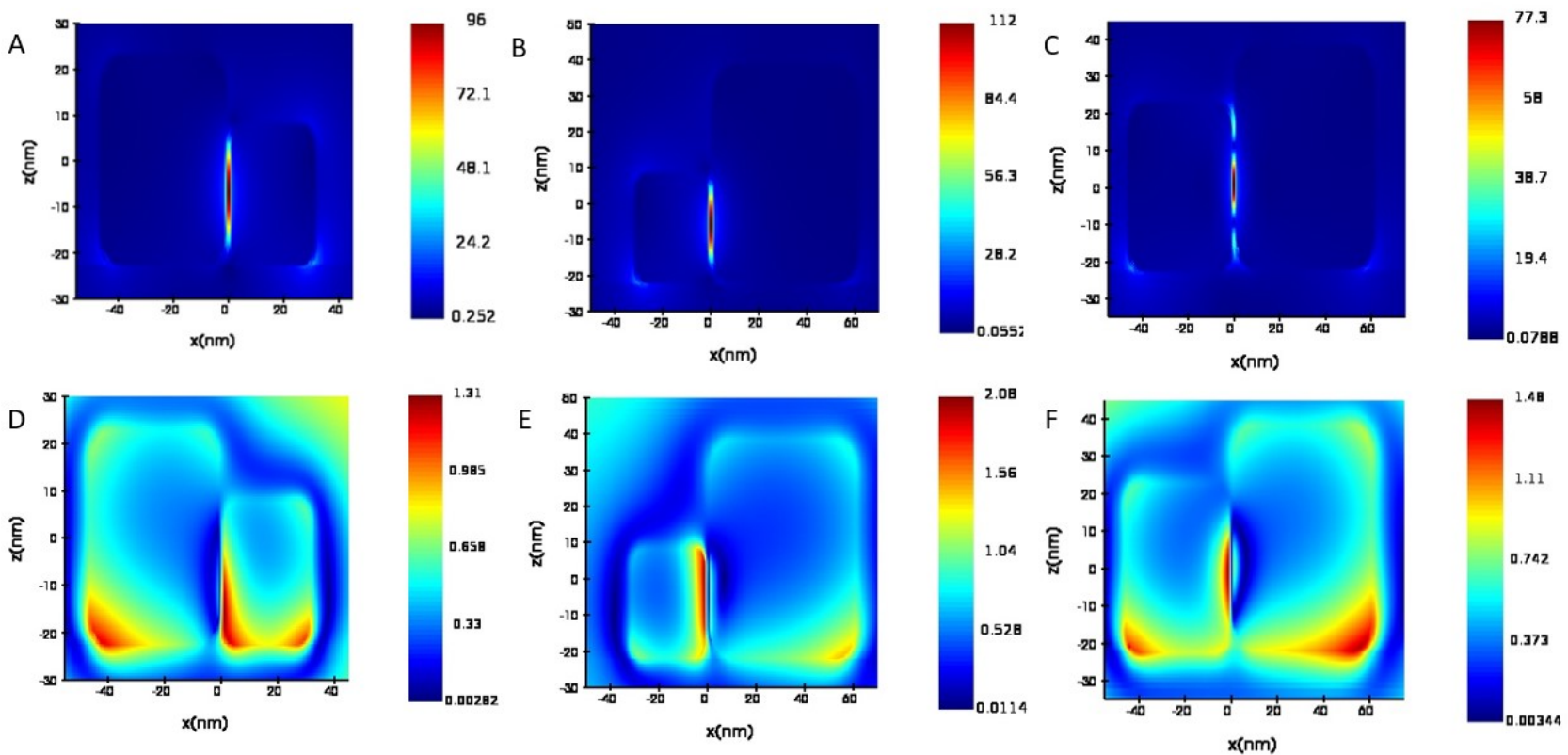

Figure 6.47: EEFs of $30 \mathrm{~nm} / 45 \mathrm{~nm}, 30 \mathrm{~nm} / 60 \mathrm{~nm}$ and $45 \mathrm{~nm} / 60 \mathrm{~nm}$ DS dimers at $568 \mathrm{~nm}$ calculated with $0^{\circ}$ polarized Light (AC) and $90^{\circ}$ polarized light (D-F). A $2 D$ Y plane was used for the calculations with $0^{\circ}$ and $90^{\circ}$ polarized Light. The coloured axis to the left of the images indicates the magnitude of the EEF.
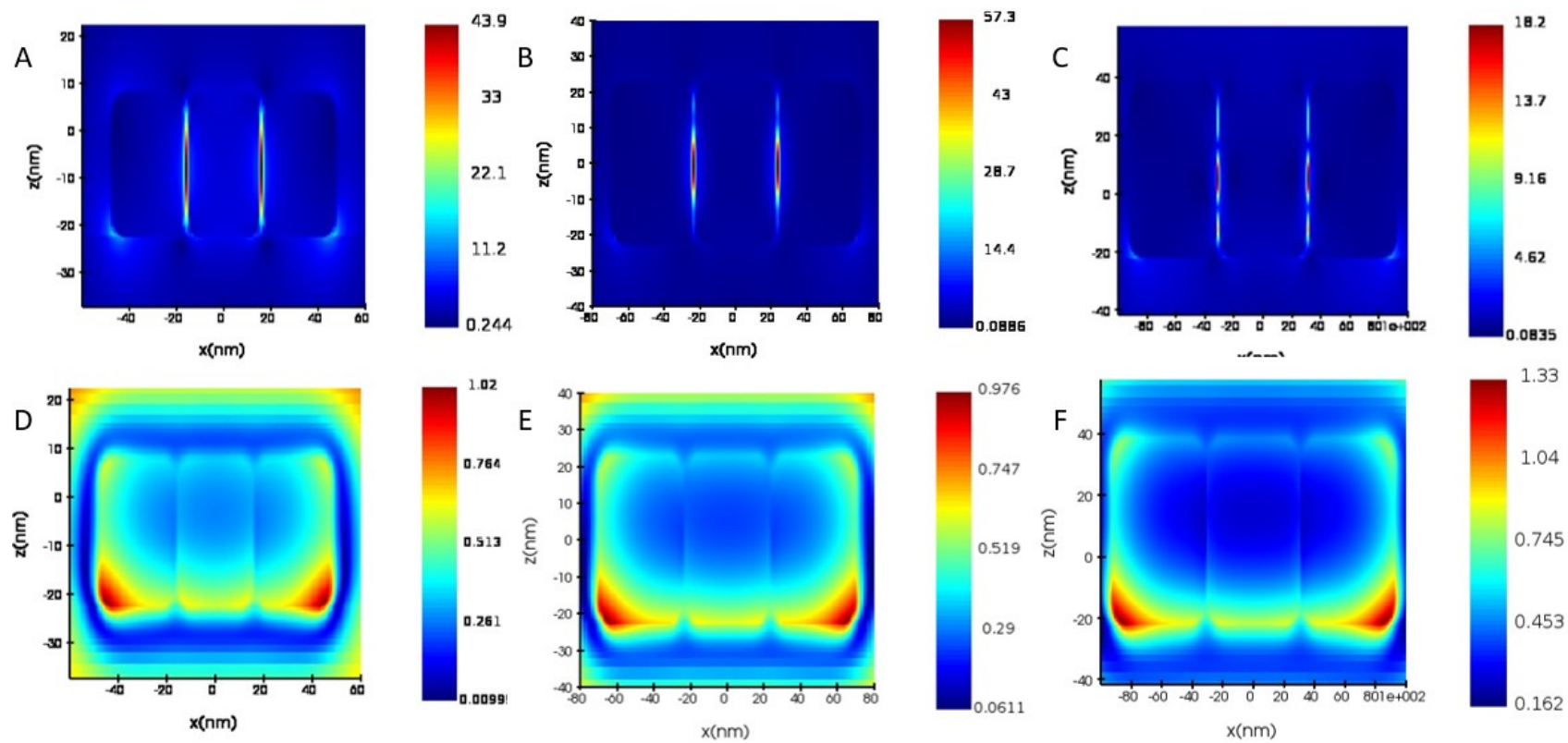

Figure 6.48: EEFs of row trimers with $30 \mathrm{~nm}, 45 \mathrm{~nm}$ and $60 \mathrm{~nm}$ cubes at $568 \mathrm{~nm}$ calculated with $0^{\circ}$ polarized light (A-C) and $90^{\circ}$ polarized light (D-F). A $2 D$ Y plane was used for the calculations with $0^{\circ}$ polarized light and $90^{\circ}$ polarized light. The coloured axis to the left of the images indicates the magnitude of the EEF. 

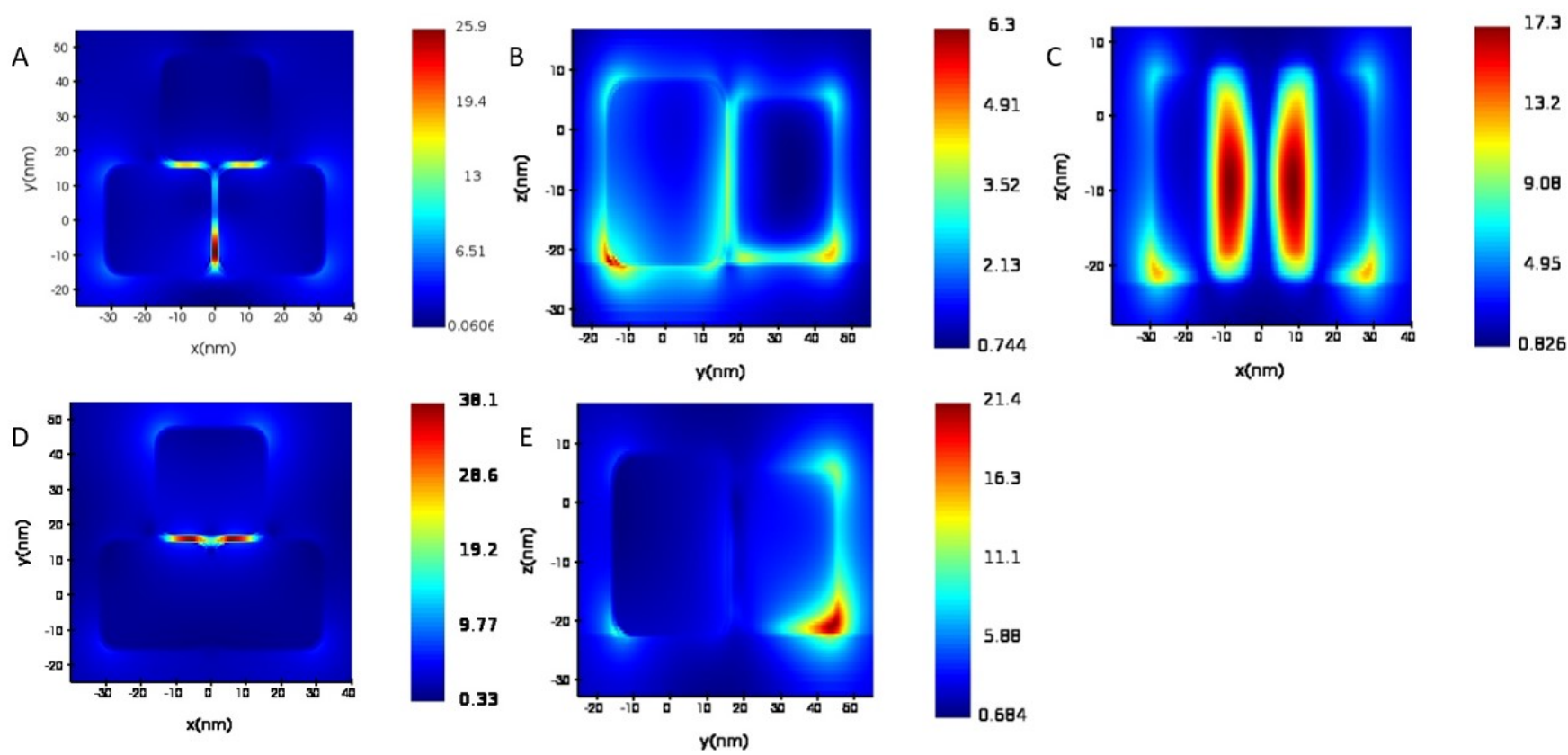

Figure 6.49: EEF of $30 \mathrm{~nm}$ triangle trimer at $568 \mathrm{~nm}$. A-C show the respective $Z, X-R$ and $Y$ planes calculated with $0^{\circ}$ polarized light and $D$ and $E$ show the $Z$ and $X-R$ planes calculated with $90^{\circ}$ polarized light. The coloured axis to the left of the images indicates the magnitude of the EEF.
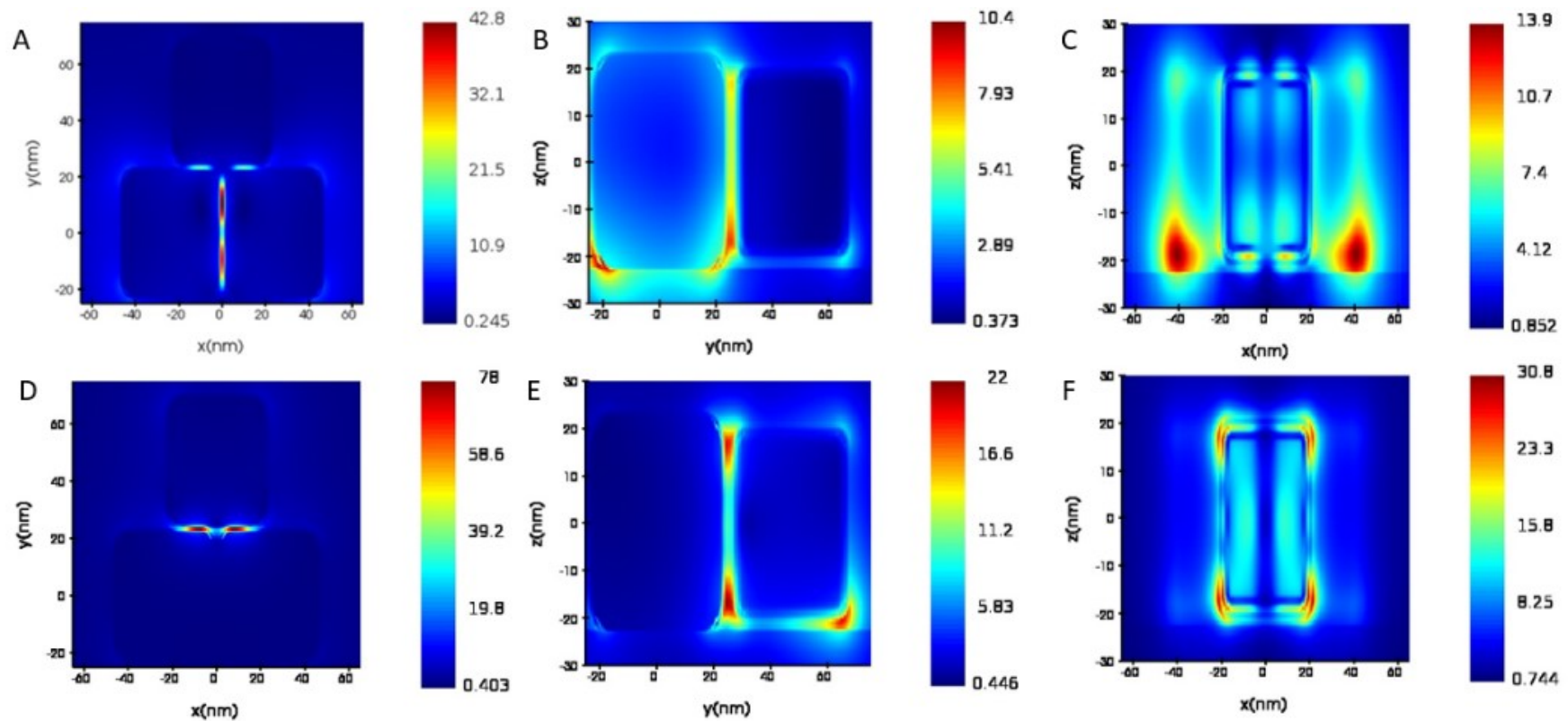

Figure 6.50: EEF of $45 \mathrm{~nm}$ triangle trimer at $568 \mathrm{~nm}$. A-C show the respective $Z, X-R$ and $Y$ planes calculate with $0^{\circ}$ Polarized Light and D-E show the $Z, X-R$ and $Y$ planes calculated with $90^{\circ}$ Polarized Light. The coloured axis to the left of the images indicates the magnitude of the EEF. 

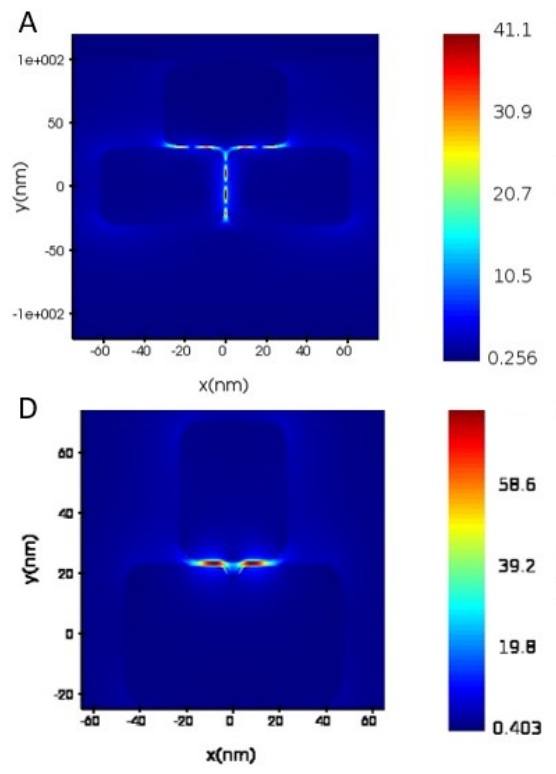
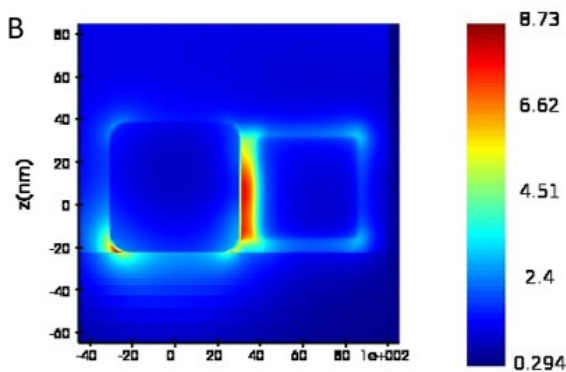

$\mathrm{v}(\mathrm{nm})$

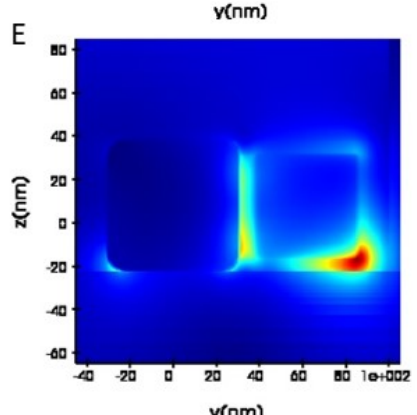

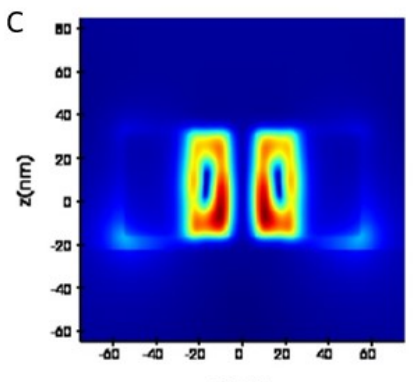

$x(\mathrm{~nm})$

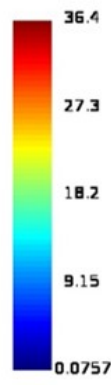

Figure 6.51: EEF of $60 \mathrm{~nm}$ triangle trimer at $568 \mathrm{~nm}$. A-C show the respective $Z, X-R$ and $Y$ planes calculate with $0^{\circ}$ polarized light and $D$ and $E$ show the $Z$ and $X-R$ planes calculated with $90^{\circ}$ polarized light. The coloured axis to the left of the images indicates the magnitude of the EEF.
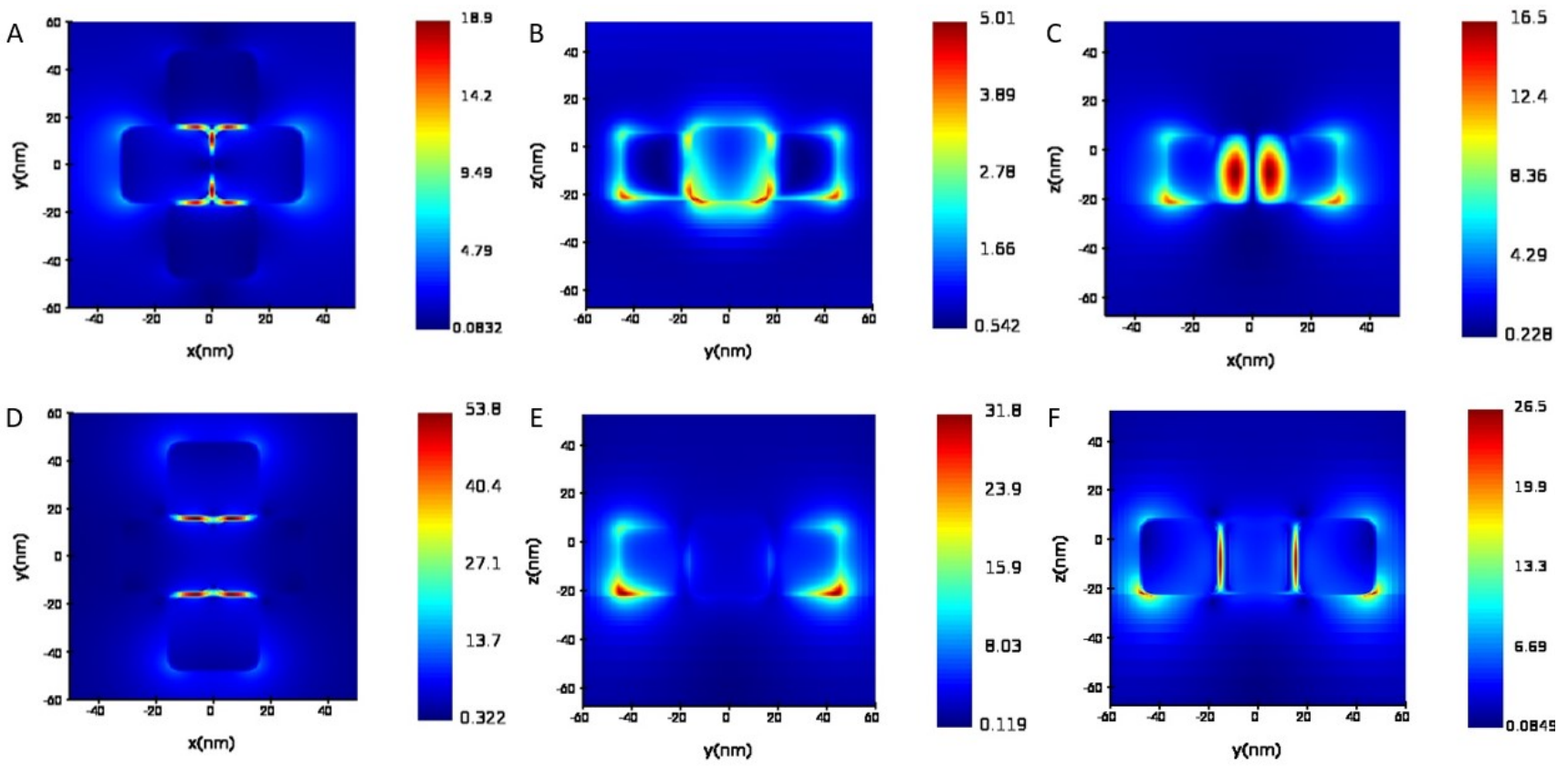

Figure 6.52: EEF of $30 \mathrm{~nm}$ AgNC diamond tetramer at $568 \mathrm{~nm}$. A-C show the respective $Z, X-R$ and $Y$ planes calculate with $0^{\circ}$ polarized light and D-F show the $Z, X-R$ and $X-M$ planes calculated with $90^{\circ}$ polarized light. The coloured axis to the left of the images indicates the magnitude of the EEF. 

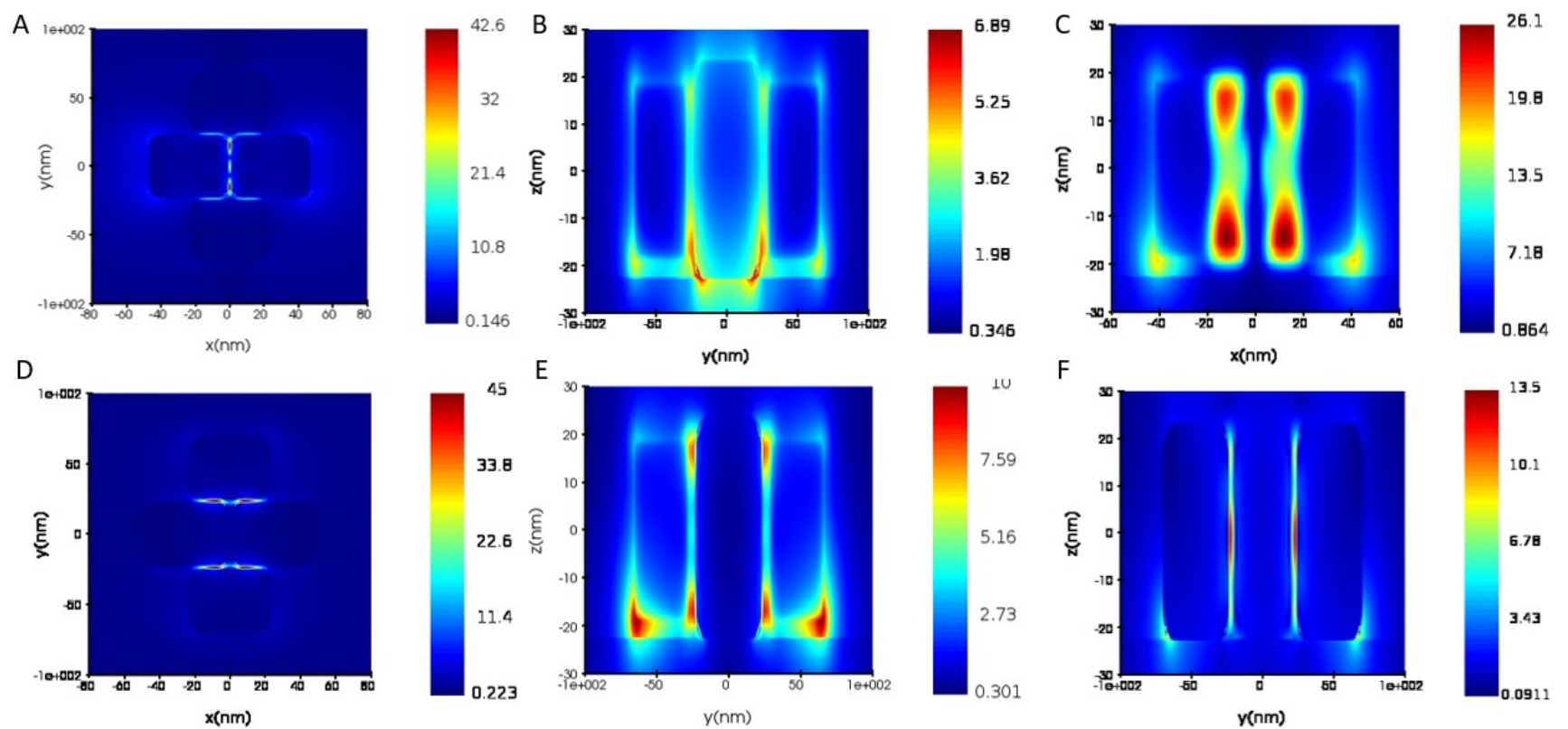

Figure 6.53: EEF of $45 \mathrm{~nm}$ AgNC diamond tetramer. A-C show the respective $Z, X-R$ and $Y$ planes calculate with $0^{\circ}$ polarized light and D-F show the $Z, X-R$ and $X-M$ planes calculated with $90^{\circ}$ polarized light. The coloured axis to the left of the images indicates the magnitude of the EEF.
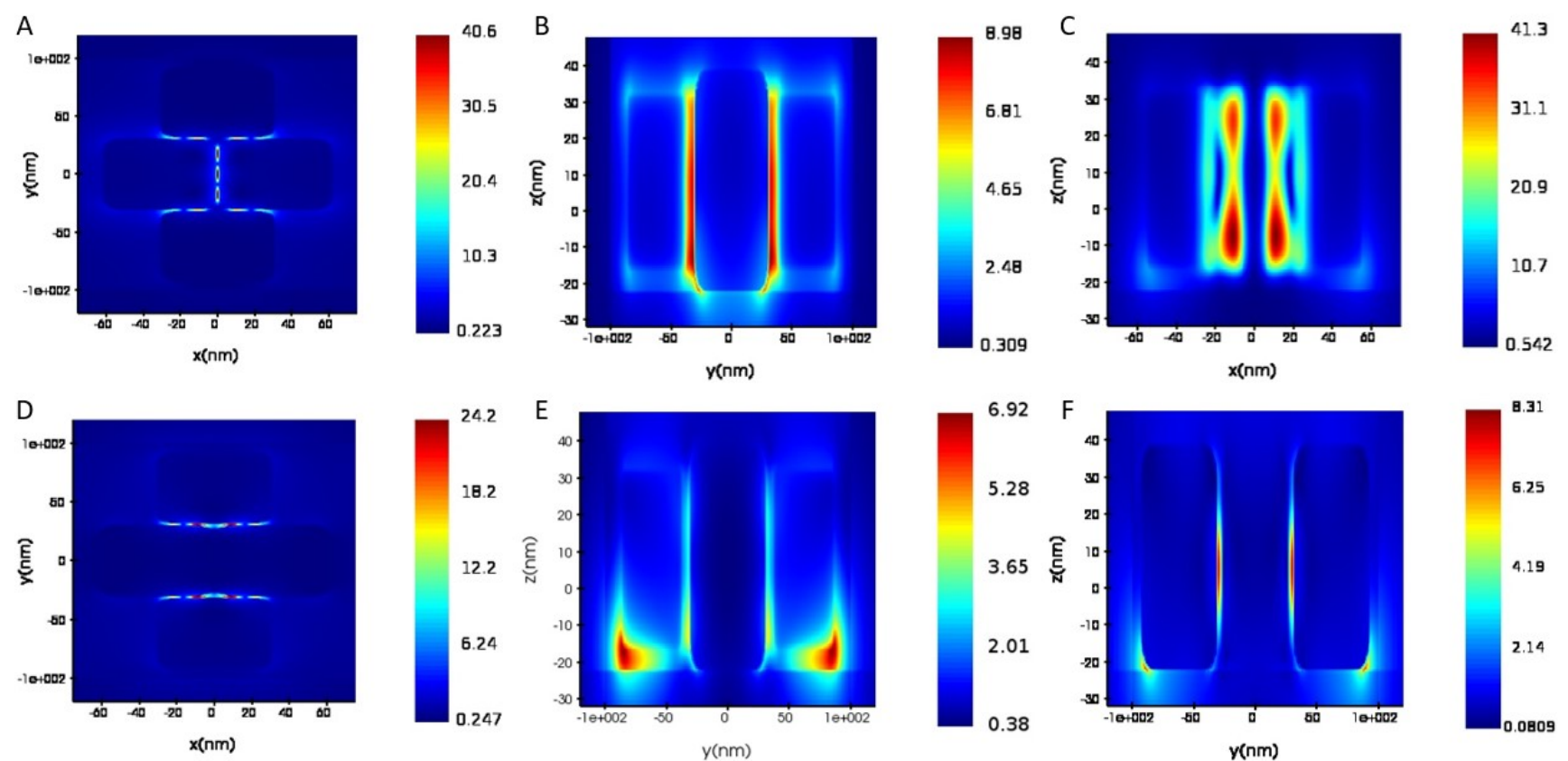

Figure 6.54: EEF of $60 \mathrm{~nm}$ AgNC diamond tetramer. A-C show the respective $Z, X-R$ and $Y$ planes calculate with $0^{\circ}$ polarized light and $D-F$ show the $Z, X-R$ and $X-M$ planes calculated with $90^{\circ}$ polarized light. The coloured axis to the left of the images indicates the magnitude of the EEF. 


\subsection{Supplementary Material for Section 3.7}

\subsubsection{E-Field Enhancement Images of Different Sizes of Nanocube Clusters for at Their Peak Absorption Wavelength}

It is important to note that in some of the EEFs below the cubes are depicted as being more rectangular in shape than square. This is due to the scale for the different axis generated by the Lumerical software.
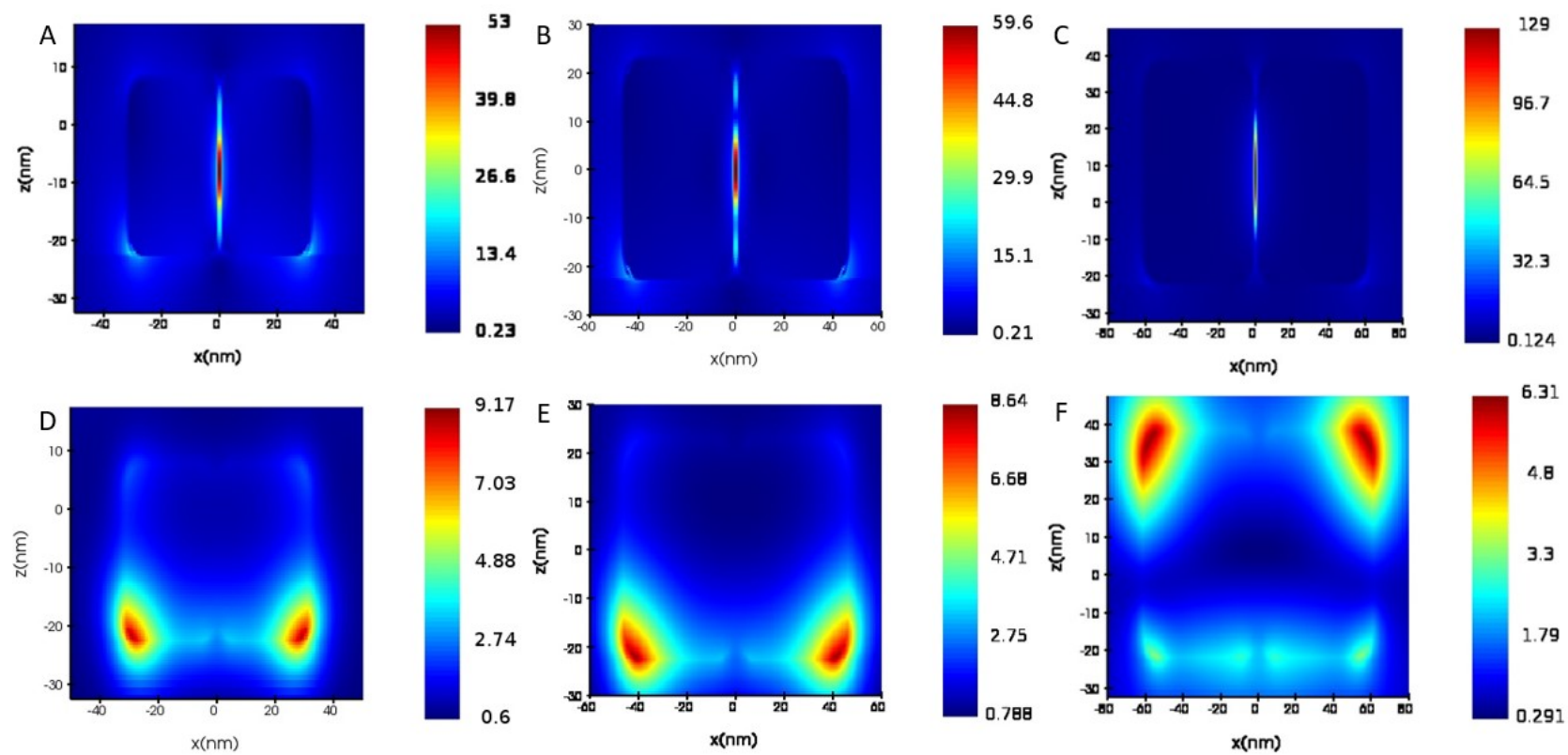

Figure 6.55: EEFs of $30 \mathrm{~nm}, 45 \mathrm{~nm}$ and $60 \mathrm{~nm}$ SS dimers calculated with $0^{\circ}$ polarized light (A-C) and $90^{\circ}$ polarized light (D-F). A $2 D$ Y plane was used for the calculations with $0^{\circ}$ polarized light and $90^{\circ}$ polarized light. The coloured axis to the left of the images indicates the magnitude of the EEF. The EEFs were calculated at $520 \mathrm{~nm}(A), 566 \mathrm{~nm}(B), 674 \mathrm{~nm}$ (C), $444 \mathrm{~nm}$ (D), 446 $\mathrm{nm}(\mathrm{E})$ and $398 \mathrm{~nm}(\mathrm{~F})$. 

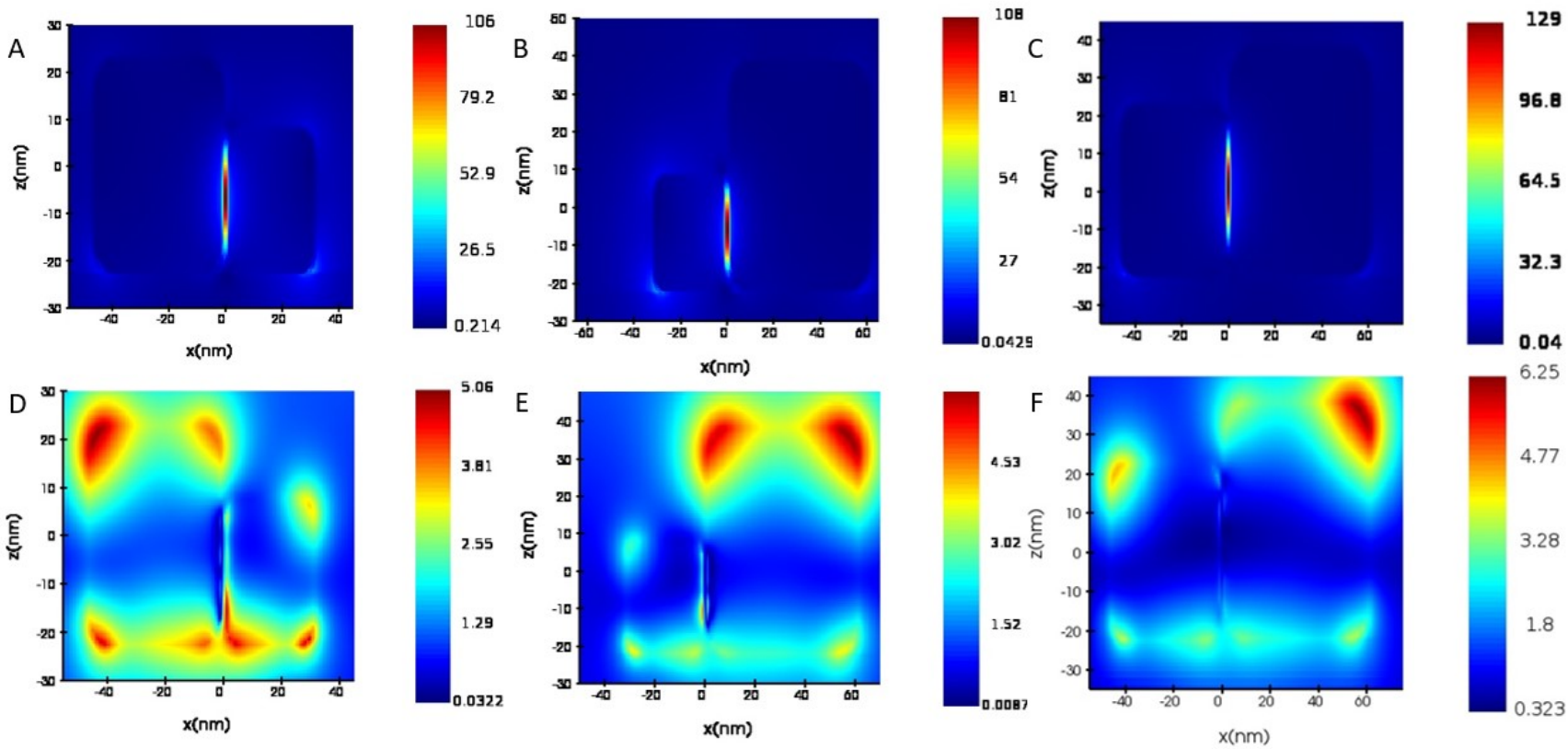

Figure 6.56: EEFs of $30 \mathrm{~nm} / 45 \mathrm{~nm}, 30 \mathrm{~nm} / 60 \mathrm{~nm}$ and $45 \mathrm{~nm} / 60 \mathrm{~nm}$ DS dimers calculated with $0^{\circ}$ polarized light (A-C) and $90^{\circ}$ polarized light (D-F). A 2D Y plane was used for the calculations with $0^{\circ}$ and $90^{\circ}$ polarized light. The coloured axis to the left of the images indicates the magnitude of the EEF. The EEFs were calculated at $558 \mathrm{~nm}(A), 576 \mathrm{~nm}(B), 622 \mathrm{~nm}(C), 404 \mathrm{~nm}$ (D), $404 \mathrm{~nm}$ (E) and $400 \mathrm{~nm}$ (F).
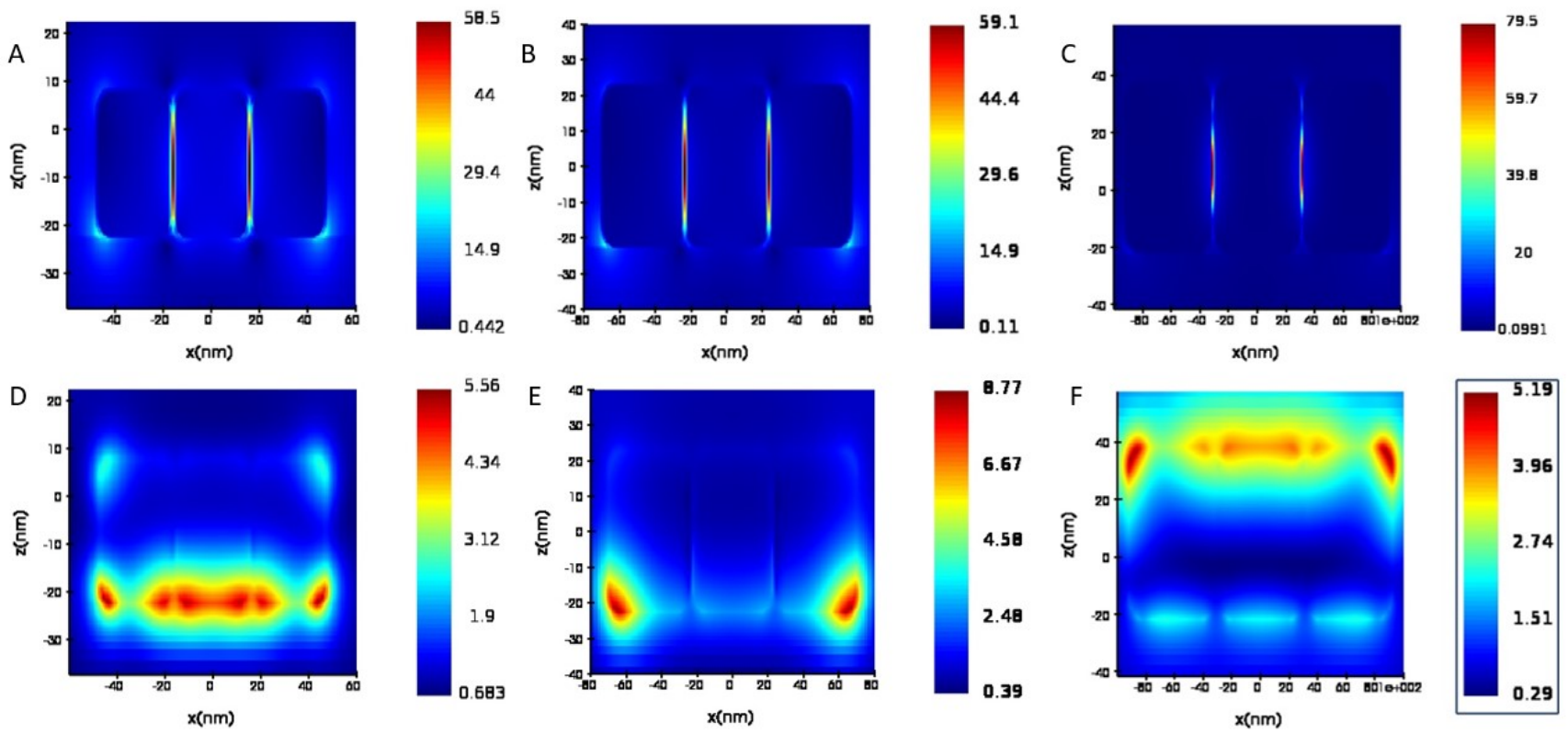

Figure 6.57: EEFs of $30 \mathrm{~nm}, 45 \mathrm{~nm}$ and $60 \mathrm{~nm}$ row trimers calculated with $0^{\circ}$ polarized light (A-C) and $90^{\circ}$ polarized light (D-F). A 2D Y plane was used for the calculations with $0^{\circ}$ polarized light and $90^{\circ}$ polarized light. The coloured axis to the left of the images indicates the magnitude of the EEF. The EEFs were calculated at $600 \mathrm{~nm}(A), 688 \mathrm{~nm}(B), 660 \mathrm{~nm}(C), 418 \mathrm{~nm}(D), 444$ $\mathrm{nm}(\mathrm{E})$ and $372 \mathrm{~nm}(\mathrm{~F})$. 

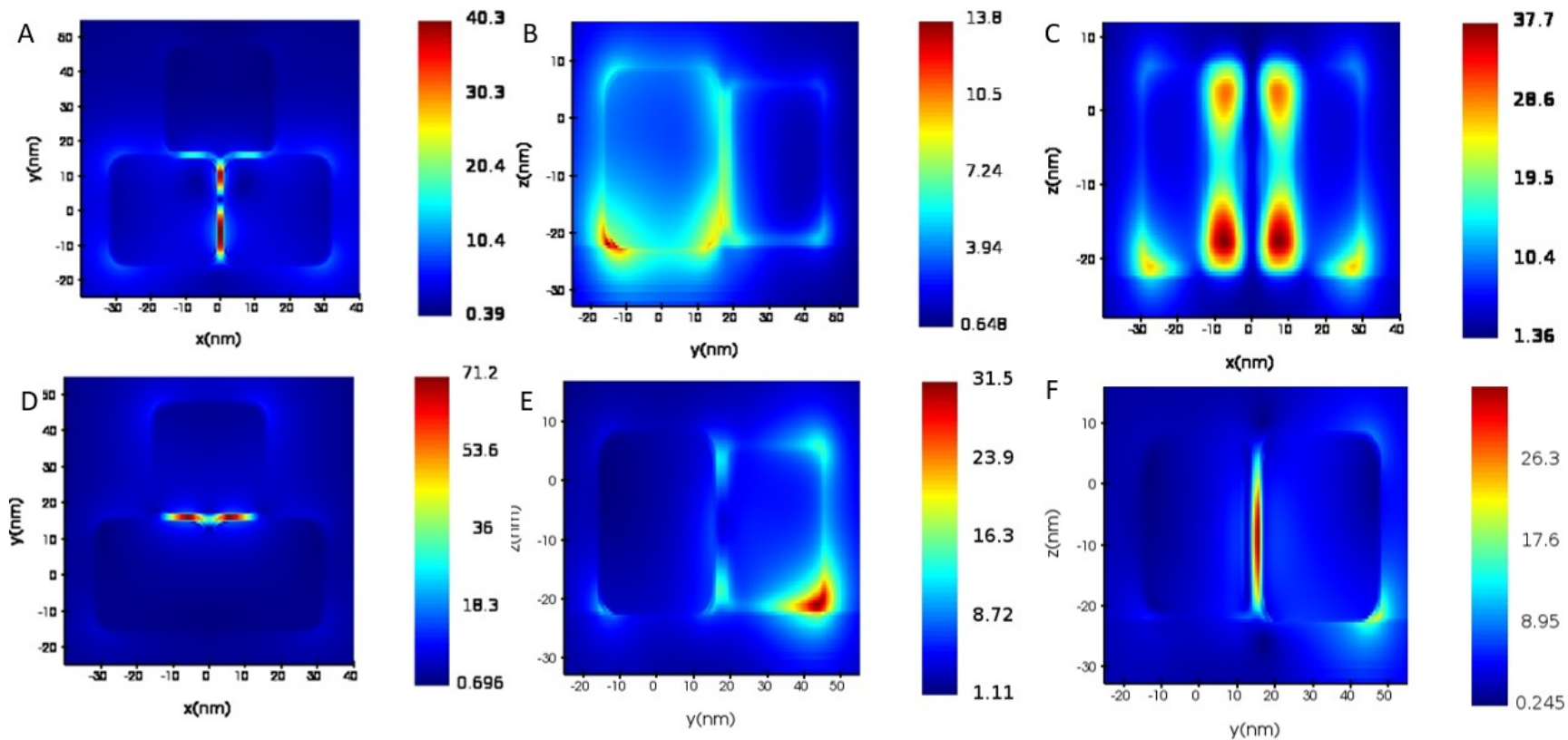

Figure 6.58: EEF of $30 \mathrm{~nm}$ triangle trimer. A-C show the respective $Z, X-R$ and $Y$ planes calculated with $0^{\circ}$ polarized light at 514 $\mathrm{nm}$ and $D-F$ show the $Z, X-R$ and planes calculated with $90^{\circ}$ polarized light at $538 \mathrm{~nm}$. The coloured axis to the left of the images indicates the magnitude of the EEF.
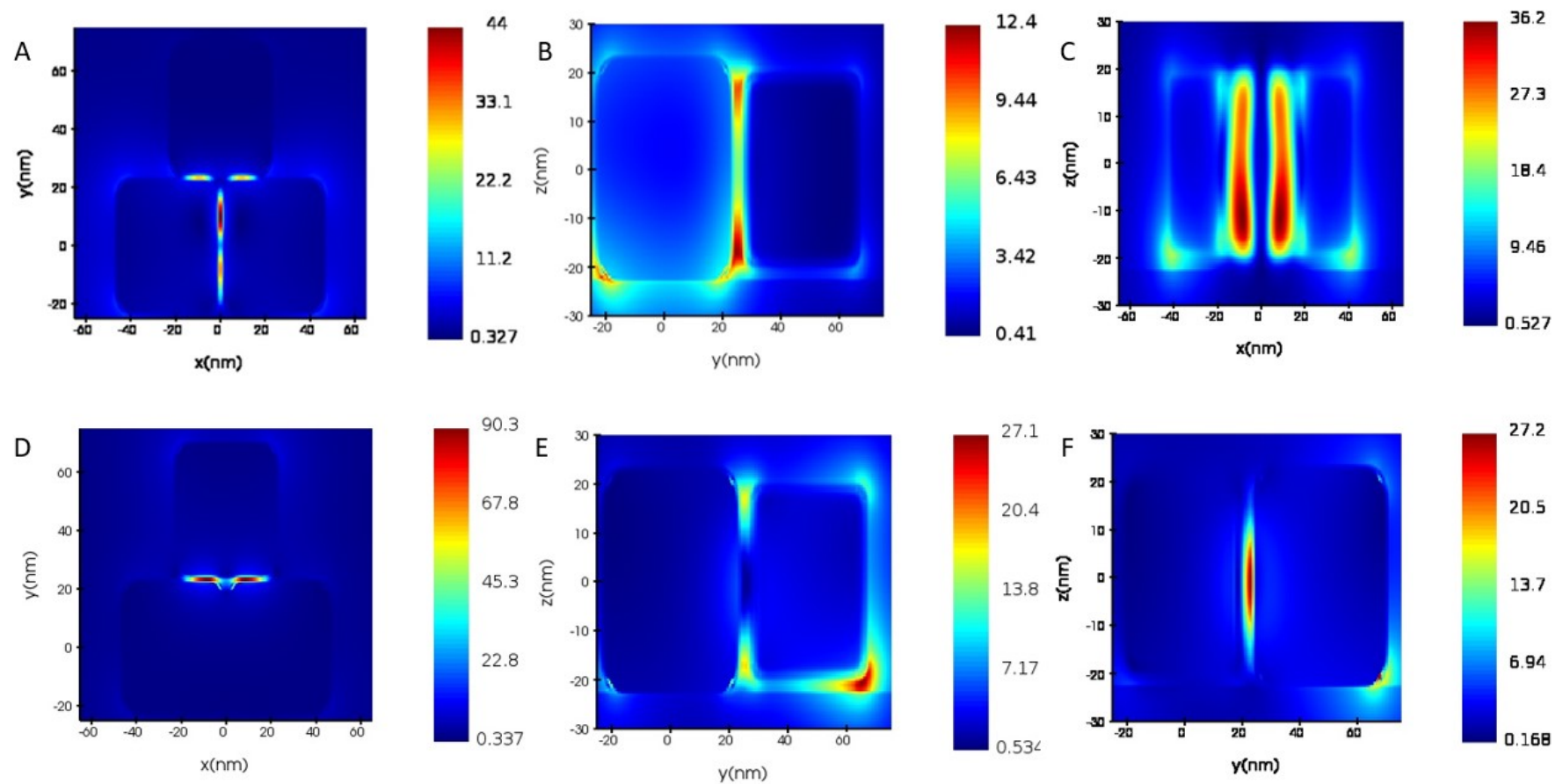

Figure 6.59: EEF of $45 \mathrm{~nm}$ triangle trimer. A-C show the respective $Z, X-R$ and $Y$ planes calculated with $0^{\circ}$ polarized light at 558 $\mathrm{nm}$ and $D-F$ show the $Z, X-R$ and planes calculated with $90^{\circ}$ polarized light at $594 \mathrm{~nm}$. The coloured axis to the left of the images indicates the magnitude of the EEF. 

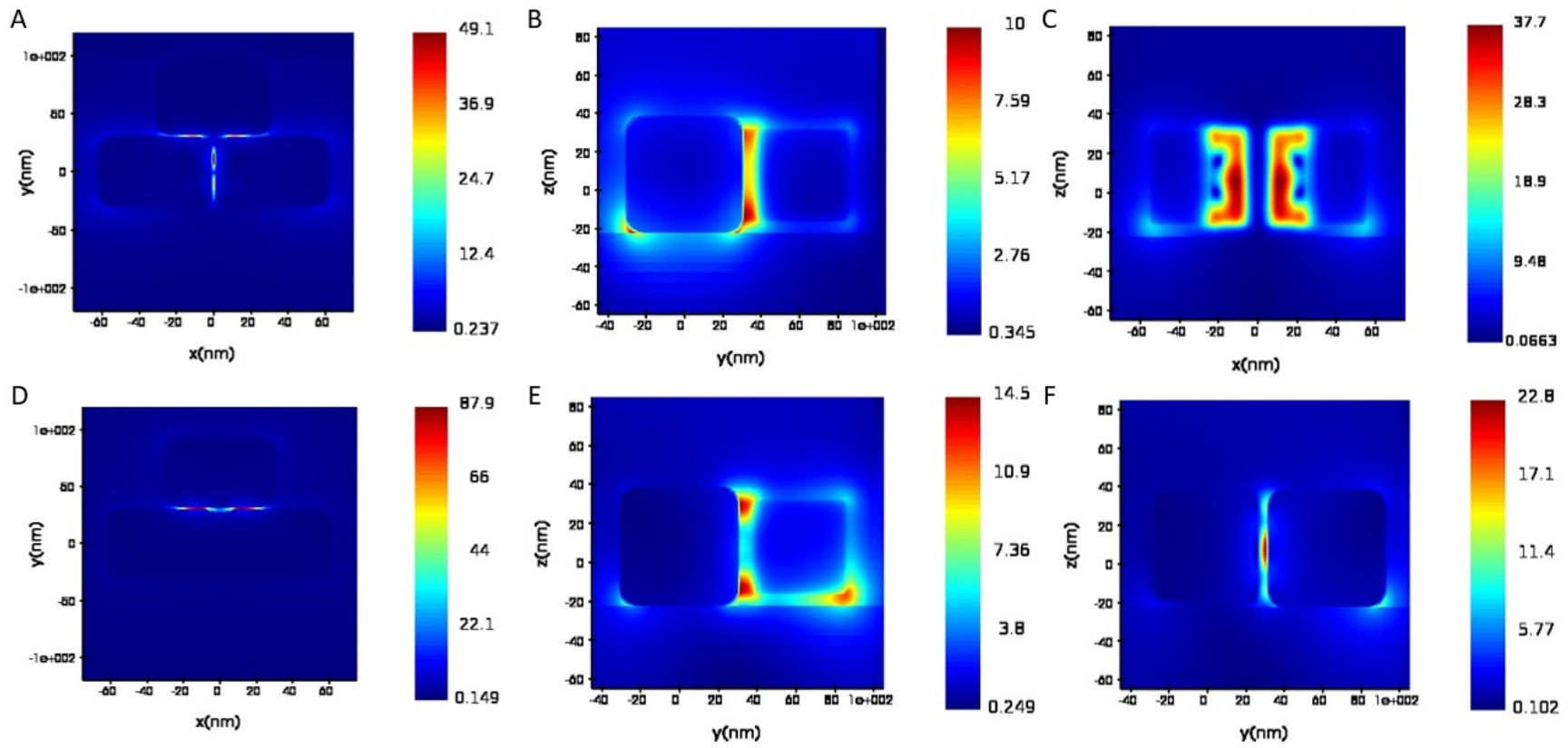

Figure 6.60: EEF of $60 \mathrm{~nm}$ triangle trimer. A-C show the respective $Z, X-R$ and $Y$ planes calculated with $0^{\circ}$ polarized light at 596 $\mathrm{nm}$ and $D-F$ show the $Z, X-R$ and planes calculated with $90^{\circ}$ polarized light at $626 \mathrm{~nm}$. The coloured axis to the left of the images indicates the magnitude of the EEF.
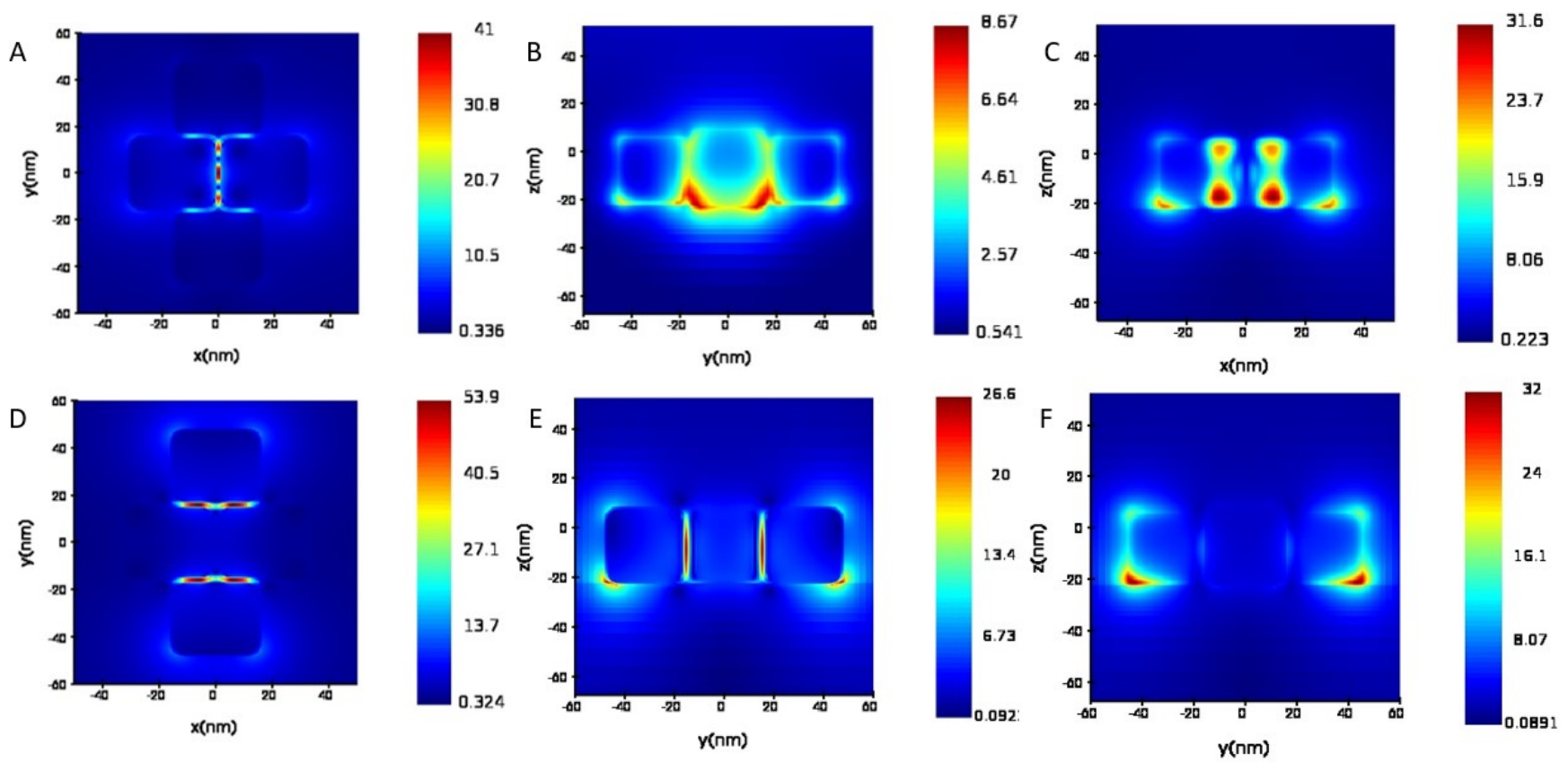

Figure 6.61: EEF of $30 \mathrm{~nm}$ diamond tetramer. A-C show the respective $Z, X-R$ and $Y$ planes calculated with $0^{\circ}$ polarized light at $502 \mathrm{~nm}$ and $D-F$ show the $Z, X-R$ and planes calculated with $90^{\circ}$ polarized light at $570 \mathrm{~nm}$. The coloured axis to the left of the images indicates the magnitude of the EEF. 

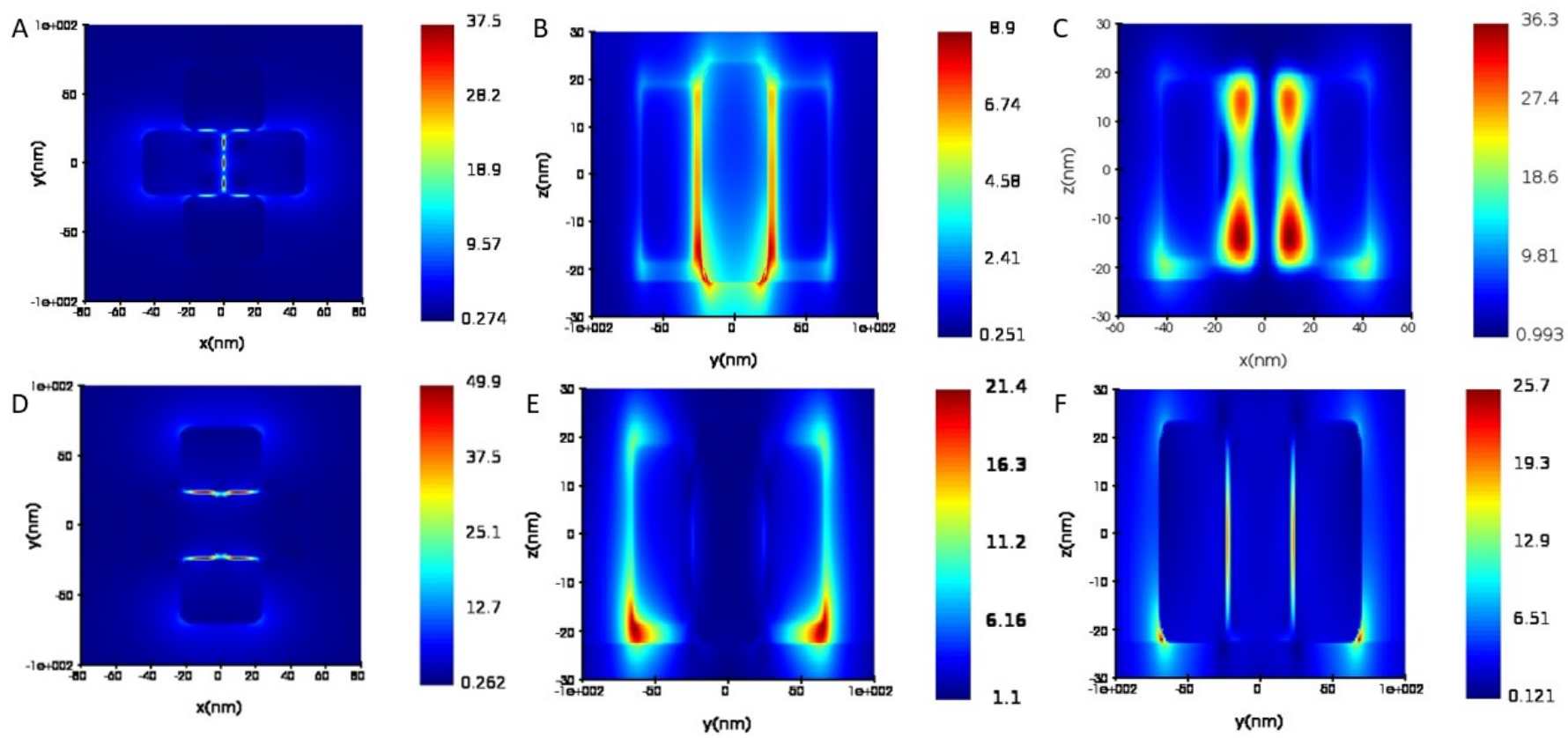

Figure 6.62: EEF of $45 \mathrm{~nm}$ diamond tetramer. A-C show the respective $Z, X-R$ and $Y$ planes calculated with $0^{\circ}$ polarized light at $544 \mathrm{~nm}$ and $D-F$ show the $Z, X-R$ and planes calculated with $90^{\circ}$ polarized light at $650 \mathrm{~nm}$. The coloured axis to the left of the images indicates the magnitude of the EEF.
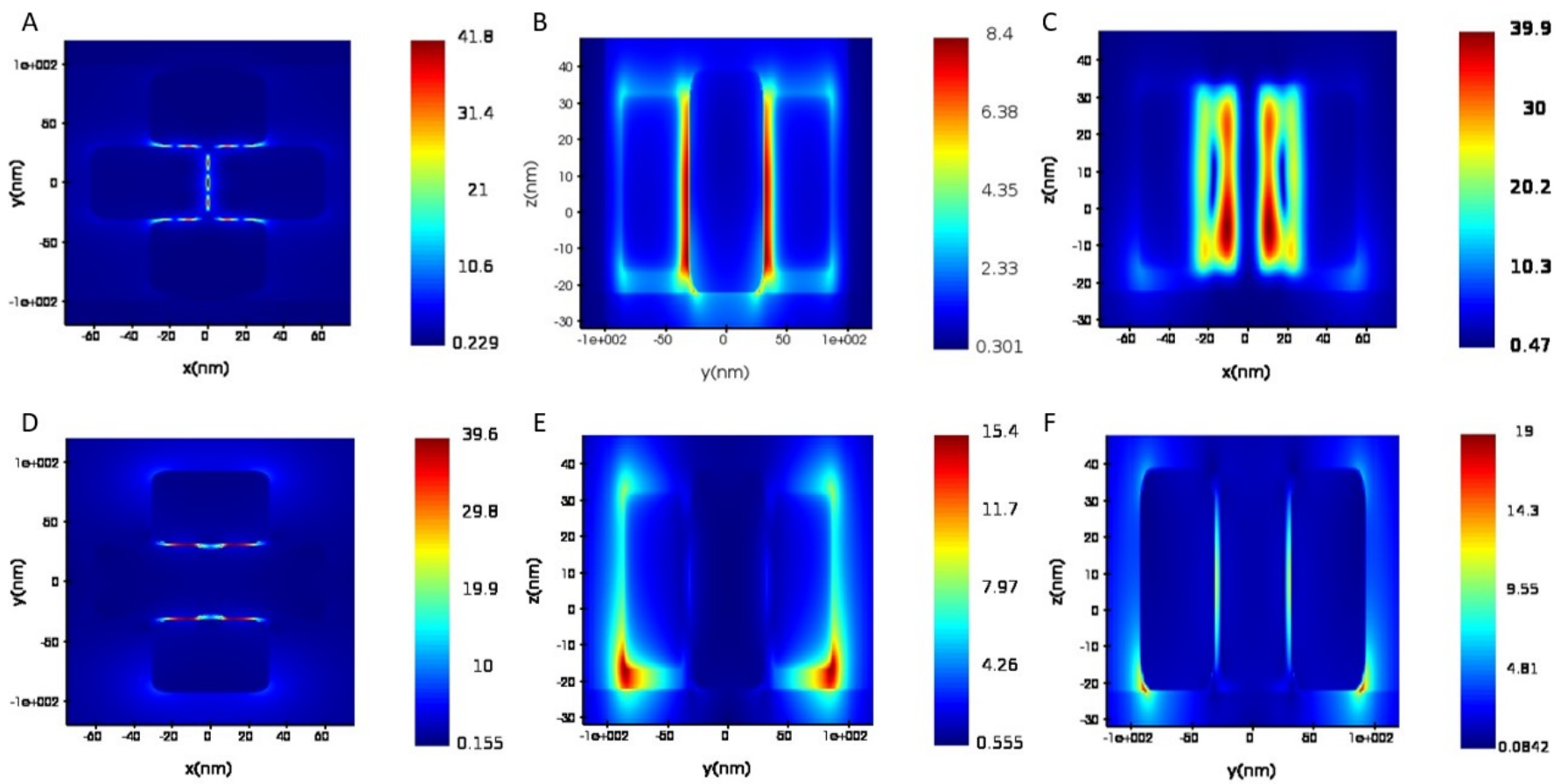

Figure 6.63: EEF of $60 \mathrm{~nm}$ diamond tetramer. A-C show the respective $Z, X-R$ and $Y$ planes calculated with $0^{\circ}$ polarized light at $558 \mathrm{~nm}$ and $D-F$ show the $Z, X-R$ and planes calculated with $90^{\circ}$ polarized light at $740 \mathrm{~nm}$. The coloured axis to the left of the images indicates the magnitude of the EEF. 